University of Louisville ThinkIR: The University of Louisville's Institutional Repository

Electronic Theses and Dissertations

$5-2013$

\title{
Consequences of four-point, strap-type wheelchair tiedown and occupant restraint system practices on wheelchair passenger safety in fixed-route transit.
}

Zdravko Salipur

University of Louisville

Follow this and additional works at: https://ir.library.louisville.edu/etd

Part of the Mechanical Engineering Commons

\section{Recommended Citation}

Salipur, Zdravko, "Consequences of four-point, strap-type wheelchair tiedown and occupant restraint system practices on wheelchair passenger safety in fixed-route transit." (2013). Electronic Theses and Dissertations. Paper 2269.

https://doi.org/10.18297/etd/2269

This Doctoral Dissertation is brought to you for free and open access by ThinkIR: The University of Louisville's Institutional Repository. It has been accepted for inclusion in Electronic Theses and Dissertations by an authorized administrator of ThinkIR: The University of Louisville's Institutional Repository. This title appears here courtesy of the author, who has retained all other copyrights. For more information, please contact

thinkir@louisville.edu. 


\title{
CONSEQUENCES OF FOUR-POINT, STRAP-TYPE WHEELCHAIR TIEDOWN AND OCCUPANT RESTRAINT SYSTEM PRACTICES ON WHEELCHAIR PASSENGER SAFETY IN FIXED-ROUTE TRANSIT
}

\author{
By \\ Zdravko Salipur \\ B.S., University of Louisville, 2005 \\ M.Eng., University of Louisville, 2008 \\ A Dissertation \\ Submitted to the Faculty of the \\ J.B. Speed School of Engineering of the University of Louisville \\ in Partial Fulfillment of the Requirements \\ for the Degree of \\ Doctor of Philosophy \\ Department of Mechanical Engineering \\ University of Louisville \\ Louisville, Kentucky
}

May 2013 



\title{
CONSEQUENCES OF FOUR-POINT, STRAP-TYPE WHEELCHAIR TIEDOWN AND OCCUPANT RESTRAINT SYSTEM PRACTICES ON WHEELCHAIR PASSENGER SAFETY IN FIXED-ROUTE TRANSIT
}

\author{
By \\ Zdravko Salipur \\ B.S., University of Louisville, 2005 \\ M.Eng., University of Louisville, 2008
}

A Dissertation Approved on

April 16, 2013

by the following Dissertation Committee:

Gina Bertocci, PhD

Dissertation Director

Karen Frost Bertocci, PhD

Patricia Karg, MSE

Mary Ellen Buning, PhD

Glen Prater, $\mathrm{PhD}$ 


\section{DEDICATION}

I dedicate this dissertation to my parents, Nermina and Ranko Salipur. They have sacrificed so much and have instilled in me a love for education and learning. For this I will forever be grateful. 


\section{ACKNOWLEDGMENTS}

Special thanks go to my dissertation advisor Dr. Gina Bertocci. Her mentorship helped me grow tremendously on both a professional and personal level. Dr. Bertocci constantly challenged me and her guidance and dedication to my success was and continues to be invaluable. Dr. Bertocci helped me discover a passion for research. She also inspired me to be rigorous and tough, all while striving to be the best human being possible.

I am also very thankful to the other members of my dissertation committee: Tricia Karg, Dr. Glen Prater, Dr. Karen Frost Bertocci, and Dr. Mary Ellen Buning. Thank you all for serving on my dissertation committee and providing very valuable support and input.

I would like to thank my ever-supporting wife, Francesca Rinaldo Salipur without whom I would have never been able to complete this work. Her encouragement and love got me though the darkest of times when there was no light at the end of the tunnel.

Special thanks to the Transit Authority of River City (TARC), Assistive Technology

Clinic at Frazier Rehab, and the entire University of Michigan Transportation Research Institute (UMTRI) staff for their collaboration and support. Thanks to my colleagues Raymond D’Souza, Angela Thompson, Craig Smalley, and Nathan Brown for all of their help on this project. This research was supported by a grant from the National Institute on Disability and Rehabilitation Research (NIDRR), Switzer Fellowship Program (Grant \# H133F100032). The opinions expressed herein are those of the author and do not necessarily represent those of the funding agency. 


\begin{abstract}
CONSEQUENCES OF FOUR-POINT, STRAP-TYPE WHEELCHAIR TIEDOWN AND OCCUPANT RESTRAINT SYSTEM PRACTICES ON WHEELCHAIR PASSENGER SAFETY IN FIXED-ROUTE TRANSIT

Zdravko Salipur
\end{abstract}

April 16, 2013

Many wheelchair users rely upon fixed route public transportation using large accessible transit vehicles (LATVs) for independent transportation to and from work, healthcare appointments and leisure activities. A substantial number of these wheelchair users may not be able to transfer from their wheelchair to a motor vehicle seat during transit. It is necessary to afford these wheelchair users the same level of safety as occupants seated in motor vehicle seats. Therefore, the Americans with Disabilities Act (ADA) requires that LATVs be equipped with wheelchair securement and occupant restraint systems (WTORS). The four-point tiedowns and occupant restraints are the primary means of WTORS on LATVs. Recent research has shown extremely high disuse and misuse rates for this wheelchair and tiedown occupant restraint system (WTORS). However, to-date, no studies have investigated the underlying causes of WTORS disuse and misuse. Perhaps more importantly, the consequences of WTORS misuse and disuse for wheelchair passengers aboard LATVs have not been determined. This study had three major phases aimed at describing consequences of WTORS misuse and disuse for wheelchair passengers on 
LATVs: I) A case series of adverse events involving wheelchair seated passengers on LATVs, II) Driving experiments using a wheelchair seated anthropomorphic testing device onboard a LATV, III) Development, validation and verification of a computer simulation of a wheelchair passenger onboard a LATV. This study revealed that the vast majority of wheelchair related instabilities are associated with minor wheelchair excursions. We also found when WTORS are properly used, the wheelchair passenger does not appear to be at increased risk of injury, while WTORS disuse and misuse are associated with a high frequency of wheelchair passenger ejection from the wheelchair, especially during an emergency braking maneuver. We found that a manual wheelchair brake can play a major role in limiting wheelchair excursions when WTORS are not properly used and that lateral wheelchair excursions can be limited by the folded-seat side barrier under the window. 
TABLE OF CONTENTS

PAGE

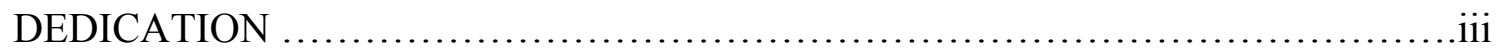

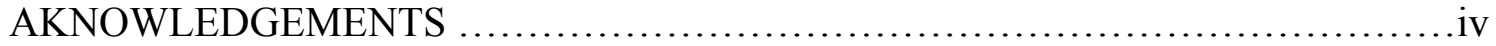

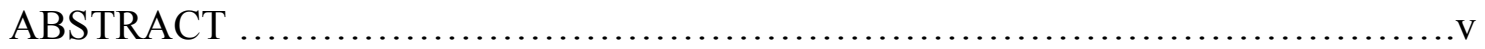

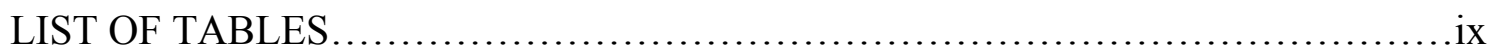

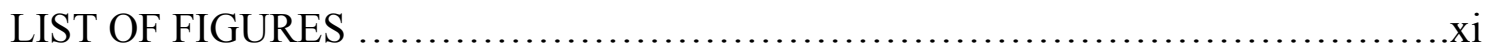

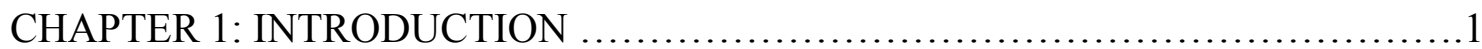

CHAPTER 2: INVESTIGATION OF WHEELCHAIR INSTABILITY DURING

TRANSPORT IN LARGE ACCESSIBLE TRANSIT VEHICLES ...................10

CHAPTER 3: INFLUENCE OF TRANSIT BUS NORMAL DRIVING CONDITIONS

ON WHEELCHAIR OCCUPANTS AND WTORS LOADS .......................39

CHAPTER 4: KINETIC ENVIRONMENT OF A MANUAL WHEELCHAIR SEATED PASSENGER ONBOARD A LARGE ACCESSIBLE TRANSIT VEHICLE DURING

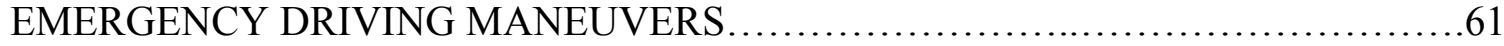

CHAPTER 5: MANUAL WHEELCHAIR EXCURSIONS UNDER VARYING WHEELCHAIR BRAKE MOUNTING HARDWARE CONDITIONS DURING LARGE ACCESSIBLE TRANSIT VEHICLE NORMAL DRIVING. .89

CHAPTER 6: DEVELOPMENT AND VALIDATION OF COMPUTER SIMULATION MODEL OF AN ADULT MANUAL TRANSIT WHEELCHAIR WITH A SEATED OCCUPANT IN A LARGE ACCESSIBLE TRANSIT VEHICLE UNDER EMERGENCY BRAKING CONDITIONS 
CHAPTER 7: PARAMETRIC SENSITIVITY ANALYSIS USING COMPUTER SIMULATION MODEL OF AN ADULT MANUAL TRANSIT WHEELCHAIR WITH A SEATED OCCUPANT IN A LARGE ACCESSIBLE TRANSIT VEHICLE UNDER EMERGENCY BRAKING ............................................. 141

CHAPTER 8: SUMMARY AND CONCLUSIONS ................................163

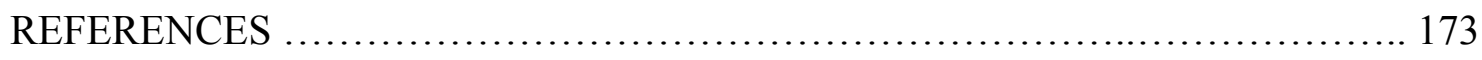

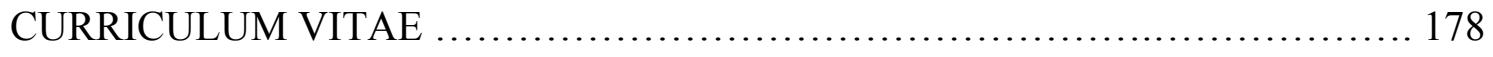




\section{LIST OF TABLES}

CHAPTER 2 TABLES

PAGE

1. Proportion of instability cases by wheelchair type in WoFT database.............17

2. Summary data for severe instability cases.................................22

\section{CHAPTER 3 TABLES}

1. Experimental test set up WTORS measurements. 45

\section{CHAPTER 4 TABLES}

1. Test Matrix of four-point, strap-type WTORS configurations used in emergency LATV driving....................................................67

2. Experimental test set up WTORS measurements..........................69

3. Outcome measures recorded during LATV emergency driving maneuvers with proper WTORS use (configuration C)................................80

4. Outcome measures recorded during LATV emergency driving maneuvers with WTORS misuse (configuration B) .................................... 81

5. Outcome measures recorded during LATV emergency driving maneuvers with WTORS disuse (configuration A) ....................................... 82

\section{CHAPTER 5 TABLES}

1. Total wheelchair excursions (distance wheelchair moved) for each trial across both brake mounting hardware conditions. 


\section{CHAPTER 6 TABLES}

1. List of outcome measures used in model validation.......................... 120

2. Summary of Statistical Tests and Test Criteria for Model Validation Assessment

3. Statistical comparison summary of model and experimental outcome measure time histories

\section{CHAPTER 7 TABLES}

1. Parameters varied during sensitivity analysis with validated computer simulation model

2. Outcome measures monitored during sensitivity analysis

3. Parametric sensitivity analysis results with peak outcome measure values. Green shading indicates model baseline values, red shading indicates overall maximum outcome measure values, and yellow shading indicates either ATD ejection from wheelchair or wheelchair/ATD excursions beyond the securement station boundaries in the model. N.O. indicates outcome measure values that were not obtainable for that particular run (e.g. ATD ejection could not yield relevant ATD excursions, rear tiedown loading could not be reported when zero rear tiedowns were used, etc.)

4. Sensitivity indices (SI) for continuous variable parameters. SI is the ratio of the percent change in outcome measure to the percent change in input parameter. The highest SI for each outcome measure is indicated in a red box...

\section{CHAPTER 8 TABLES}

1. Recommendations for stakeholders invested in wheelchair passenger safety in LATV transit.. 


\section{LIST OF FIGURES}

1. In-vehicle 6-camera LATV video surveillance system. This LATV was equipped with cameras to include views from the front windshield, front and rear of the LATV interior, and front and rear doors, as well as the wheelchair securement station...

2. Tree-diagram showing direction of excursion, number of tiedowns used, and occupant restraint use for 57 wheelchair instability events categorized as minor instability

3. Tiedown and occupant restraint configurations by wheelchair type in minor instability cases.

4. Case 1: Unsecured power wheelchair enters aisle during routine LATV transit, while sitting ambulatory passenger lifts foot to prevent injury and limit power wheelchair movement. (View from LATV rear) ........................................ 24

5. Diagram showing unsecured power wheelchair rotating laterally into aisle during routine LATV transit (Case 1)

6. Case 2: Manual wheelchair passenger and wheelchair rotated into aisle as LATV performed a right turn. Wheelchair passenger stabilized himself by placing both feet on the floor and using his right arm to grab the folding-seat while an ambulatory passenger used her foot to prevent additional lateral excursion of the wheelchair. (View from LATV front) .26

7. Diagram showing manual wheelchair and wheelchair passenger rotating laterally into the aisle during a routine right turn. Note: orientation of this diagram is $180^{\circ}$ opposite of Figure 6 camera view. 26

8. Case 3: Power wheelchair passenger ejected from wheelchair during normal LATV braking. Wheelchair passenger struck the bulkhead in front of the securement station and fell to the floor complaining of a fractured leg. (View from LATV rear)........28 
9. Diagram showing power wheelchair passenger ejected from power wheelchair during an apparent routine braking maneuver. During ejection, (1) the wheelchair passenger struck the LATV bulkhead directly in front of the right securement station, and then (2) fell to the floor in the aisle.

\section{CHAPTER 3 FIGURES}

1. LATV route driven during testing.

2. A. Typical test setup of 50th percentile ATD seated in manual wheelchair with four tiedowns and lap and shoulder belt applied. Low-g accelerometers were mounted in the ATD head, chest, and pelvis as well as on the LATV floor (at securement station). Loadcells were used to measure webbing tension in the tiedowns and lap and shoulder belts

B. Test setup measurements obtained during test setup

3. Typical LATV acceleration time histories for acceleration, braking, left turn, and right turn maneuvers under normal driving conditions

4. Mean peak resultant accelerations for ATD and LATV for acceleration $(n=87)$, braking $(\mathrm{n}=81)$, left turn $(\mathrm{n}=10)$, and right turn maneuvers $(\mathrm{n}=10)$ under normal driving conditions. (Error bars indicate $95 \%$ confidence intervals)

5. Mean peak WTORS loading for acceleration (from standstill) maneuvers under normal LATV driving conditions. (Error bars indicate $95 \%$ confidence intervals).

6. Mean peak WTORS loading for braking (to standstill) maneuvers under normal LATV driving conditions. (Error bars indicate 95\% confidence intervals).

7. Mean peak WTORS loading for right turn maneuvers under normal LATV driving conditions. (Error bars indicate 95\% confidence intervals).

8. Mean peak WTORS loading for left turn maneuvers under normal LATV driving conditions. (Error bars indicate $95 \%$ confidence intervals) .......................50

\section{CHAPTER 4 FIGURES}

1. Emergency driving course conducted on a closed driving course 
2. A. Typical test setup of 50th percentile ATD seated in manual wheelchair with four tiedowns and lap and shoulder belt applied. Low-g accelerometers were mounted in the ATD head, chest, and pelvis as well as on the LATV floor (at securement station). Loadcells were used to measure webbing tension in the tiedowns and lap and shoulder belts.

B. Test setup measurements obtained during test setup .68

3. Test setup of 50th percentile ATD seated in manual wheelchair with lap belt used in an attempt to secure the wheelchair (routed around the seatback, WTORS configuration B). The tiedowns and shoulder belt were not applied.

4. Representative LATV acceleration time histories for acceleration, braking, left turn, right turn, braking/left-turn, braking/right-turn, and lane change maneuvers under emergency driving conditions

5. Mean peak LATV resultant accelerations (independent of WTORS configuration) for acceleration $(\mathrm{n}=7)$, braking $(\mathrm{n}=5)$, left turn $(\mathrm{n}=6)$, right turn $(\mathrm{n}=6)$, braking/left-turn $(\mathrm{n}=4)$, braking/right-turn $(\mathrm{n}=5)$, and lane change $(\mathrm{n}=4)$ maneuvers under emergency driving conditions. (Error bars indicate $95 \%$ confidence intervals) ..................73

6. Mean peak resultant accelerations for ATD and wheelchair (proper WTORS use, configuration C) for acceleration ( $\mathrm{n}=3)$, braking $(\mathrm{n}=3)$, left turn $(\mathrm{n}=3)$, right turn $(\mathrm{n}=3)$, braking/left-turn $(\mathrm{n}=2)$, braking/right-turn $(\mathrm{n}=3)$, and lane change $(\mathrm{n}=2)$ maneuvers under emergency driving conditions. (Error bars indicate $95 \%$ confidence intervals;

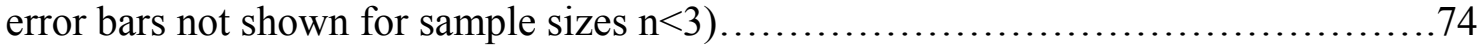

7. Mean peak WTORS loading during LATV emergency acceleration $(n=3$, error bars indicate $95 \%$ confidence intervals) ............................................. 75

8. Mean peak WTORS loading during LATV emergency braking $(\mathrm{n}=3$, error bars indicate

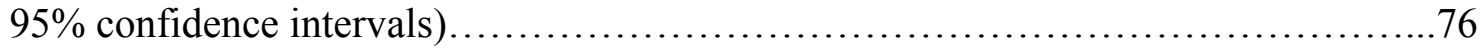

9. Mean peak WTORS loading during LATV emergency left turns $(n=3$, error bars indicate $95 \%$ confidence intervals)...........................................................

10. Mean peak WTORS loading during LATV emergency right turns $(n=3$, error bars

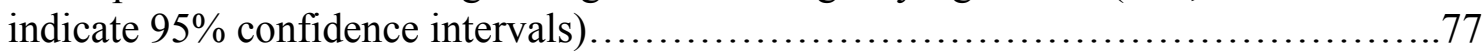

11. Mean peak WTORS loading for LATV emergency braking/left-turn maneuvers ( $\mathrm{n}=2$,

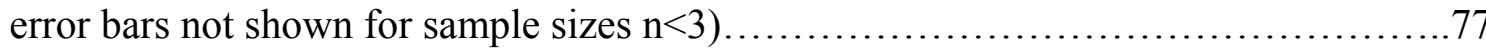

12. Mean peak WTORS loading for LATV emergency braking/right-turn maneuvers $(\mathrm{n}=3$, error bars indicate $95 \%$ confidence intervals) ...................................... 78 
13. Mean peak WTORS loading for LATV emergency lane change maneuvers $(\mathrm{n}=2$, error

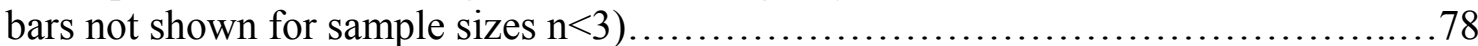

\section{CHAPTER 5 FIGURES}

1. A. Test setup of 50th percentile ATD seated in manual wheelchair with lap belt used in an attempt to secure the wheelchair (routed around the seatback). The tiedowns and shoulder belt were not applied.

B. Test setup of 50th percentile ATD seated in manual wheelchair with lap belt used in an attempt to secure the wheelchair (routed around the seatback). The tiedowns and shoulder belt were not applied................................................ 94

2. Design of manual wheelchair (compact scissor) brake. The brake is shown in the engaged position. To securely clamp the brake to the wheelchair frame, the two bolts were tightened to lock the position rod inside the mounting bracket. With the position rod fixed relative to the mounting bracket (and therefore the wheelchair frame), the impingement lever (attached to the position rod) was able to effectively impinge the rear wheel tire to prevent rear wheel rotation with respect to the wheelchair frame.........96

3. Wheelchair trajectory during trial 1 (brake mounting hardware loosely clamped to the wheelchair frame). The dark-shaded circular markers represent wheelchair locations (rear axle) at sequential points over trial duration. Dimensions are in inches............98

4. Wheelchair trajectory during trial 2 (brake mounting hardware loosely clamped to the wheelchair frame). The dark-shaded circular markers represent wheelchair locations (rear axle) at sequential points over trial duration. Dimensions are in inches.

5. Wheelchair trajectory during trial 3 (brake mounting hardware loosely clamped to the wheelchair frame). The dark-shaded circular markers represent wheelchair locations (rear axle) at sequential points over trial duration. Dimensions are in inches

6. Wheelchair trajectory during trial 4 (brake mounting hardware loosely clamped to the wheelchair frame). The dark-shaded circular markers represent wheelchair locations (rear axle) at sequential points over trial duration. Dimensions are in inches

7. Wheelchair trajectory during trial 5 (brake mounting hardware securely clamped to the wheelchair frame). The dark-shaded circular markers represent wheelchair locations (rear axle) at sequential points over trial duration. Dimensions are in inches...........103

8. Wheelchair trajectory during trial 6 (brake mounting hardware securely clamped to the wheelchair frame). The dark-shaded circular markers represent wheelchair locations (rear axle) at sequential points over trial duration. Distances are in inches 
9. Wheelchair trajectory during trial 7 (brake mounting hardware securely clamped to the wheelchair frame). The dark-shaded circular markers represent wheelchair locations (rear axle) at sequential points over trial duration. Distances are in inches.

10. Wheelchair trajectory during trial 8 (brake mounting hardware securely clamped to the wheelchair frame). The dark-shaded circular markers represent wheelchair locations (rear axle) at sequential points over trial duration. Dimensions are in inches

\section{CHAPTER 6 FIGURES}

1. Test setup of 50th percentile ATD seated in manual wheelchair with four tiedowns and lap and shoulder belt applied. Low-g accelerometers were mounted in the ATD head and chest, the wheelchair CG, as well as on the LATV floor (at securement station). Load cells were used to measure webbing tension in the tiedowns and lap and shoulder belts

2. Experimental LATV acceleration pulse during a LATV emergency combination braking/left-turn maneuver....

3. LATV wheelchair model (forward- facing) with 50th percentile ATD.

4. Frame Sequence of Experimental Test (Top) and Computer Simulation Model (Bottom).

5. Right rear tiedown loading: model (solid line) vs. experiment (dashed)....

6. Left rear tiedown loading: model (solid line) vs. experiment (dashed)...............125

7. Shoulder belt loading: model (solid line) vs. experiment (dashed).................. 126

8. Wheelchair resultant acceleration: model (solid line) vs. experiment (dashed)........127

9. ATD chest resultant acceleration: model (solid line) vs. experiment (dashed).........128

10. ATD head resultant acceleration: model (solid line) vs. experiment (dashed)

\section{CHAPTER 7 FIGURES}

1. LATV deceleration pulse of sedan automobile frontal impact into LATV. Pulse peak is $2 \mathrm{~g}$ (frontal crash).

2. LATV suspension stiffness range in parametric sensitivity analysis. Computer model simulated LATV suspension during an emergency braking maneuver as a rotational joint positioned at the center of the securement station platform (allowing platform fore-aft 
tilt). Joint baseline stiffness in model is depicted with a solid line. The LATV suspension stiffness was varied $\pm 18 \%$ (dashed lines) based on published acceleration differences associated with varying LATV suspensions.

3. Range of peak RRTD loading values for each parameter varied in the sensitivity analysis. Red dashed line shows model baseline value.

4. Range of peak RLTD loading values for each parameter varied in the sensitivity analysis. Red dashed line shows model baseline value.

5. Range of peak wheelchair fore/aft excursion values for each parameter varied in the sensitivity analysis. Red dashed line shows model baseline value....

6. Range of peak wheelchair lateral excursion values for each parameter varied in the sensitivity analysis. Red dashed line shows model baseline value....

7. Range of peak wheelchair fore/aft tilt values for each parameter varied in the sensitivity analysis. Red dashed line shows model baseline value.

8. Range of peak wheelchair lateral tilt values for each parameter varied in the sensitivity analysis. Red dashed line shows model baseline value. 153

9. Range of peak ATD fore/aft excursion values for each parameter varied in the sensitivity analysis. Red dashed line shows model baseline value.

10. Range of peak ATD lateral excursion values for each parameter varied in the sensitivity analysis. Red dashed line shows model baseline value. 


\section{CHAPTER 1}

\section{INTRODUCTION}

\section{Specific Aims}

Large accessible transit vehicles (LATVs) are generally considered a safe mode of transportation. Due to their large mass and low accelerations, LATVs have a low incidence of crashes and passenger fatalities ${ }^{1-4}$. However, research has shown that for persons who remain seated in their wheelchairs during transit, crash conditions are not

necessary for injuries to occur ${ }^{1-6}$. Several studies advocate that wheelchair passenger risk is greater than that of general ridership ${ }^{1,3,5,6}$, and it has been suggested that this may be due to postural instability and lack of voluntary muscle control (in some cases) ${ }^{1-3}$. A recent study found that four-point, strap-type wheelchair tiedown and occupant restraint systems (WTORS) used to secure wheelchairs and restrain wheelchair-seated passengers, are associated with high levels of disuse and misuse onboard large transit buses ${ }^{7}$. Notably, no studies have been conducted investigating the potential adverse outcomes of four-point, strap-type WTORS disuse and misuse for wheelchair passengers during routine and emergency LATV driving. The purpose of this study was to gain a better understanding of safety and injury risk related to four-point, strap-type WTORS disuse 
and misuse during routine and emergency LATV driving. The aims of this study were four-fold:

1. To characterize adverse wheelchair events that occur as a consequence of fourpoint, strap-type WTORS disuse and misuse during routine LATV driving maneuvers.

2. To characterize adverse wheelchair events that occur as a consequence of fourpoint, strap-type WTORS disuse and misuse during emergency LATV driving maneuvers.

3. To identify factors related to the four-point, strap-type WTORS, wheelchair, occupant, and driving conditions that contribute to adverse wheelchair events during LATV routine and emergency driving.

4. To provide recommendations that can be used by transit providers, WTORS manufacturers, and wheelchair manufacturers to increase passenger safety onboard LATVs.

The findings of this study identified the consequences of four-point, strap-type WTORS disuse and misuse, and provided the rationale for new transit agency policies regarding wheelchair transportation safety. The aims were achieved through three methodological phases: I) A case series of adverse events involving wheelchair passengers on LATVs, II) Driving experiments using a wheelchair seated anthropomorphic testing device (ATD) onboard a LATV, and III) Development, validation, and verification of a computer simulation of a wheelchair passenger onboard a large accessible transit vehicle. Using the model, a parametric sensitivity analysis was conducted to investigate the influence of various model parameters on wheelchair and passenger outcomes. The three phases of this study are described in six chapters (chapters $2-7)$. 


\section{Rationale}

Wheelchair users must often rely upon public transportation to access work, medical care, school and social and recreational activities. Although many wheelchair users depend upon public transport to perform activities of daily living, it has been shown that wheelchair-seated passengers often sustain serious injuries during routine and emergency driving maneuvers when using public transit ${ }^{1-6}$. Research has documented high levels of disuse and misuse when it comes to securing wheelchairs and restraining wheelchair occupants on LATVs ${ }^{7}$. It is unknown whether four-point, strap-type WTORS disuse and misuse could result in wheelchair and passenger instability and/or increased risk of tipping or falling during transit. A more complete understanding of the consequences wheelchair passengers face because of four-point, strap-type WTORS misuse and disuse is needed to improve equality in the levels of safety, usability, and independence for wheelchair-seated passengers traveling in buses.

The Americans with Disabilities Act (ADA) calls for the provision of wheelchair tiedowns and occupant restraints on LATVs, but does not require their use ${ }^{8}$. It has been shown that there is no clear understanding of ADA mandates amongst transit providers ${ }^{9}$. Accordingly, public transport agencies exhibit varying policies for WTORS usage with regard to wheelchair-occupant safety on LATVs. Individual transit agencies may require the use of four-point wheelchair tiedowns and occupant restraints (consisting of a lap and shoulder belt), the use of wheelchair tiedowns only, or the use of occupant restraints only ${ }^{10}$. Differences among transit agency safety protocols for wheelchair users may be due to the fact that many people consider LATVs to be a safe mode of transportation because of their large mass and low accelerations (low g environment) ${ }^{1,11}$. Safety on LATVs is in 
large part based on the riding experience of non-wheelchair passengers, as they are not required to use occupant restraints and many stand during transit ${ }^{2,3}$. However, it has been well documented that LATVs are not necessarily safe for wheelchair passengers since they are more likely to sustain injuries during both routine and emergency driving

maneuvers on LATVs ${ }^{3,6}$. The misuse and disuse of four-point, strap-type WTORS could substantially be contributing to decreased safety for wheelchair passengers on LATVs. Therefore, there is a need for detailed study of the consequences of four-point, strap-type WTORS disuse and misuse for wheelchair passengers onboard LATVs. Knowledge generated from this study could then be used to make informed recommendations for the standardization of safety protocols that are optimized specifically for the safety of wheelchair users on LATVs.

\section{Literature Review}

When considering the problem of wheelchair transportation safety on fixed-route transit, it is first important to examine the incidence of adverse outcomes for the general ridership. Research has shown that LATVs are a safe mode of transportation, with a low fatality incidence for all passengers. Shaw ${ }^{1}$ found that transit buses are the safest form of transportation for the general public with a yearly fatality rate of 0.01 per 100 million passenger miles. In comparison, cars had a fatality rate of 0.95 , railroad 0.1 , scheduled airlines 0.02 , and general buses 0.02 per 100 million passenger miles. ${ }^{1}$

There is evidence that LATVs are not as safe for wheelchair passengers when compared to the general public. Shaw and Gillispie ${ }^{3}$ found that wheelchair passengers have an accident rate over 350 times greater than that of ambulatory passengers. The vast 
majority of wheelchair passengers $(85 \%)$ cannot transfer to a motor vehicle seat and must remain seated in their wheelchair during transit ${ }^{10}$. Songer and Fitzgerald ${ }^{6}$ report that of the passengers that remain in their wheelchairs during transit, 14\% reported a non-crash injury, while $6.5 \%$ reported involvement in a crash (but were not necessarily injured) . Passengers who reported typically using a wheelchair as a vehicle seat had higher injury rates than those who transferred to vehicle seats; for public vehicle passengers: 5.2 vs. 0.6 injuries per 100000 mi traveled. ${ }^{6}$

The cost of wheelchair-related injuries occurring on fixed-route public transit imposes a burden on our healthcare system. Shaw and Gillispie ${ }^{3}$ found that efforts should be directed towards reducing the number of injuries caused by non-crash events, as incidents cost an average of $\$ 2500$. Another study determined that all non-crash wheelchair events were amongst the 10 most costly event types, despite having only one or two claimants per event. ${ }^{2}$ The ratio of wheelchair claims to non-wheelchair claims greater than $\$ 9000$ was found to be much higher than the ratio of wheelchair riders to general riders. $^{2}$

Crash conditions may not be necessary to incur wheelchair passenger injury and death. Specifically, adverse outcomes taking place during LATV movement occur during both routine (turning, breaking) and evasive (emergency) maneuvers and often involve tipping of the wheelchair and subsequent falling of the passenger. Frost and Bertocci found $94 \%$ of wheelchair passenger injuries occur while the LATV is moving, and 100\% of severe wheelchair passenger injuries with medical attention sought occur when LATVs are moving ${ }^{7}$. Wretstrand, et al. ${ }^{12}$ also found that most wheelchair user injuries occurred during routine driving maneuvers. According to Frost and Bertocci ${ }^{7}, 20 \%$ of 
incidents occurred under normal driving conditions (mostly vehicle turns, rest involving LATV slowing / stopping) while only $6.1 \%$ occurred during emergency maneuvers. Another study ${ }^{3}$ determined that from 35 incidents that included wheelchair riders, 25 involved the bus turning, 4 involved a sudden stop, and 4 other normal bus operations. In another part of the same study, Shaw and Gillispie ${ }^{3}$ state that $57 \%$ of all passenger injuries reviewed were due to falls under normal operating conditions and that $56 \%$ of all passenger injuries occurred during braking, half of which were during sudden braking. Shaw and Gillispie $^{3}$ also report that braking caused $85 \%$ of events without collisions. Frost and Bertocci, $2009^{7}$ found that $44 \%$ of incidents studied involved combined wheelchair and passenger tipping/falling; $20 \%$ of incidents involved passenger falling off wheelchair only. Finally, one study found that the majority of wheelchair passenger deaths occurred in non-crash LATV events and that eliminating injury in these non-crash events would reduce overall wheelchair passenger injury frequency by $50 \%{ }^{2}$

It has been acknowledged that postural instability of wheelchair passengers may put them at increased risk for adverse events, even during routine LATV maneuvers ${ }^{1}$. More importantly, however, wheelchair users may be at higher injury risk on LATVs due to misuse and disuse of wheelchair securement and occupant restraint measures. The $\mathrm{ADA}^{13}$ requires that LATVs be equipped with four-point, strap-type WTORS with specified minimum strength requirements for forward-facing wheelchair stations. However, ADA regulation allows for mandates on wheelchair securement use to be determined by individual transit authorities ${ }^{8}$. Also, under the broad non-discrimination provisions in ADA regulations, a transit provider is not permitted to mandate the use of lap and shoulder belts by wheelchair users, unless the transit provider mandates the use of 
these devices by all passengers, including those sitting in vehicle seats ${ }^{8}$. Nonetheless, wide disparities amongst transit agency WTORS policies have been documented ${ }^{9,10}$ and may indicate a vague understanding of WTORS mandates on the part of transit providers. Buning, et al. ${ }^{10}$ found that while the majority (66.7\%) of transit providers surveyed required tiedown use for transporting wheelchair passengers, only $25.6 \%$ out of those providers also required occupant restraints and $69.5 \%$ made occupant restraints optional. Almost eighteen percent (17.9\%) of transit providers make wheelchair securement optional, and out of those $90.9 \%$ have policies with optional occupant restraints for wheelchair passengers. With a variety of wheelchairs in the marketplace, bus drivers and/or wheelchair passengers are often left to approximate their best understanding of safe securement practice ${ }^{10}$. For instance, Frost and Bertocci ${ }^{7}$ characterized four-point, strap-type WTORS usage on LATVs in a metropolitan area and found that the vast majority (76\%) of wheelchairs were not secured during transport. In only $8 \%$ of cases, all four tiedowns were used to secure the wheelchair. The occupant restraints were used only $20 \%$ of the time; in all of these cases, only the lap belt was used. Frost and Bertocci ${ }^{7}$ found that misuse of the lap belt was common (44\% of cases), and consisted of the LATV operators attempting to secure the wheelchair by wrapping the lap belt around the wheelchair seatback.

When it comes to the usage of four-point, strap-type WTORS on LATVs, confusion is also evident on the part of wheelchair passengers ${ }^{9,10}$. With regard to requesting securement, Buning, et al. ${ }^{10}$ found that $39 \%$ of wheelchair passengers never asked to be secured ( $85 \%$ reported that the bus driver seemed unwilling to take the time required, and $45.8 \%$ said that the bus driver appeared "rushed"). Out of the passengers 
surveyed, only $36.6 \%$ routinely asked to be secured. Interestingly, studies also show that LATV operator attitudes (possibly affected by unclear transit provider policy) influence whether or not wheelchair occupants choose to secure their wheelchair. Fitzgerald, et al. ${ }^{5}$ found poor public transportation driver attitudes towards assisting wheelchair users with proper wheelchair securement. Frost and Bertocci ${ }^{7}$ report that verbal disputes between LATV operators and wheelchair passengers with respect to WTORS use often lead to wheelchair users refusing tiedowns or occupant restraints.

There is evidence to suggest that wheelchair passengers may face increased risks because of WTORS disuse and misuse. Shaw and Gillispie ${ }^{3}$ found that personal injuries were caused in part by wheelchair passengers being improperly secured. The National Highway Traffic Safety Administration (NHTSA) published a research note ${ }^{4}$ showing that $35 \%$ of injuries involving wheelchair users were due to improper securement or a lack of securement. Of these injuries, $7 \%$ of wheelchair users who were improperly secured or not secured were seriously injured; $17 \%$ of these cases resulted in deaths. The elderly were most likely to be injured or die with $73 \%$ of the wheelchair users injured being at least 60 years old. Frost and Bertocci ${ }^{7}$ showed that for all incidents involving wheelchair passengers on LATVs, 7\% of incidents occurred with no WTORS used, and $4 \%$ of incidents occurred with an unknown number of WTORS used. Although there is an indication that WTORS disuse and misuse may increase injury risk of wheelchair passengers on LATVs, no studies have been conducted to characterize adverse events that could occur as a result of WTORS disuse and misuse.

To summarize, LATVs are generally considered a safe mode of transportation, but may not be as safe for wheelchair passengers because of possible wheelchair and 
postural instabilities ${ }^{1-3,6,7,9-11}$. ADA regulation may not be stringent enough since it only requires that four-point, strap-type WTORS be provided, but does not mandate their use ${ }^{8}$. This causes confusion on the part of transit authorities and wheelchair passengers, as evident by a wide disparity of policies amongst transit agencies with respect to fourpoint, strap-type WTORS use ${ }^{9,10}$. There is evidence that wheelchair passengers face increased risks because of four-point, strap-type WTORS disuse and misuse ${ }^{1-4,7}$ but no studies have yet been conducted to describe the direct consequences of four-point, straptype WTORS disuse and misuse. Recommendations for change in transit authority policies can only be made if there is a better understanding and documentation of adverse outcomes related to four-point, strap-type WTORS disuse and misuse on LATVs. 
CHAPTER $2^{\dagger}$

\title{
INVESTIGATION OF WHEELCHAIR INSTABILITY DURING TRANSPORT IN LARGE ACCESSIBLE TRANSIT VEHICLES
}

\begin{abstract}
Overview
Even though large accessible transit vehicles (LATVs or fixed-route intra-city buses) are generally considered a safe mode of transportation, it has been shown that LATVs may not be as safe for persons who remain seated in their wheelchairs during transit. There are documented disparities between transit provider practices regarding the use of four-point, strap-type wheelchair tiedown and occupant restraint systems (WTORS) to secure wheelchairs and restrain wheelchair-seated passengers during LATV transit. Additionally, recent research suggests that there are high levels of WTORS disuse and misuse onboard LATVs. The purpose of this study was to gain a better understanding of wheelchair and wheelchair-seated passenger instability related to WTORS disuse and misuse when traveling in LATVs.
\end{abstract}

\footnotetext{
${ }^{\dagger}$ Work in this chapter published in peer reviewed journal article: Salipur Z., Frost K., Bertocci G. Investigation of Wheelchair Instability During Transport in Large Accessible Transit Vehicles. Journal of Rehabilitation Research and Development. 49(6). 2012.
} 
This study presents a retrospective review of 295 video surveillance records of wheelchair passenger trips on LATVs. Wheelchair trips involving disuse and misuse of WTORS during transit were quantified and categorized based on WTORS configurations during transit. Adverse events involving wheelchair and wheelchair passenger instability during transit were further categorized based on severity, type, and direction. Three adverse events involving severe wheelchair and/or passenger instability were examined in greater detail.

The results of this study showed that $20.3 \%$ of the records reviewed involved wheelchair related adverse events, with $95 \%$ of those being minor instabilities and $5 \%$ severe instabilities. When proportionally adjusted, scooters were most likely to be involved in instabilities, followed by manual and power wheelchairs. This study also showed that in a majority of instability cases the number of tiedowns used to secure the wheelchair was zero and the lap belt was not used to restrain the wheelchair passenger properly. In many instability cases the lap belt was misused in an attempt to secure the wheelchair whereas the shoulder belt was never used.

\section{Introduction}

Large accessible transit vehicles (LATVs), commonly known as fixed-route intracity buses, are generally considered a safe mode of transportation with a low fatality incidence of all passengers ${ }^{1}$. This is due in part to overall vehicle mass and the slow speed of LATV travel associated with intra-city routes. Despite this relatively safe environment, Shaw and Gillispie ${ }^{3}$ found that wheelchair passengers have an accident rate over 350 times greater than that of ambulatory passengers. Shaw ${ }^{2}$ estimated that the ratio 
of wheelchair passenger injury claims relative to the wheelchair passenger population is much higher than the claims ratio for the general population, indicating higher likelihood of wheelchair passengers to incur injuries. The U.S. National Highway Transportation Safety Administration (NHTSA) reported that $35 \%$ of injuries and deaths that occurred between 1990-1995 involving wheelchair users were due to improper or no securement of the wheelchair, and $17 \%$ of these incidents occurred in LATVs ${ }^{4}$.

In general, it is recommended that wheelchair passengers transfer to a vehicle seat, provided they have adequate postural stability and the opportunity to utilize occupant restraints. However, the vast majority of surveyed wheelchair passengers $(85 \%)$ remain seated in their wheelchair during transit, which may put them at increased risk of injury ${ }^{10}$. Songer, et al. (2004) found that passengers who remained seated in their wheelchair during transit experienced higher injury rates (5.2 passenger injuries per 100,000 miles) than those who transferred to a motor vehicle seat ( 0.6 passenger injuries per 100,000 miles) ${ }^{6}$.

It is necessary to afford wheelchair passengers the same level of transportation safety as the general population. The Americans with Disabilities Act (ADA) ${ }^{13}$ requires that LATVs be equipped with a wheelchair securement device and lap and shoulder belts. A common method to secure the wheelchair and restrain the wheelchair occupant is with the use of four-point, strap type wheelchair tiedown and occupant restraint systems (WTORS). The correct use of such WTORS is dependent upon two components. Firstly, the wheelchair should be secured using 4 tiedown straps attached at appropriate securement points on the wheelchair. Two tiedowns attach to the front of the wheelchair and two to the rear of the wheelchair. Secondly, the wheelchair passenger should be 
restrained with the lap and shoulder belts so that they make contact with the anterior of the pelvis near the upper thighs and with the shoulder and chest, respectively ${ }^{14}$. Although the ADA requires the provision of a wheelchair securement device and occupant restraints (lap and shoulder belts), it does not mandate the use of such systems ${ }^{8}$. Individual transit agencies are left to establish policies requiring the use of wheelchair tiedowns. Additionally, the ADA does not require mandatory use of lap and/or shoulder belt restraints for wheelchair-seated passengers unless the same mandate has been made for the general ridership ${ }^{8}$. As a result, there are disparities between transit agency policies with respect to WTORS use ${ }^{10}$.

Disuse and misuse of WTORS have been shown to place wheelchair-seated passengers at greater risk of injury. Shaw and Gillispie ${ }^{3}$ identified misuse as a factor contributing to wheelchair passengers injuries. In our previous study ${ }^{7}$, we reviewed video surveillance records of WTORS usage on LATVs and found that the majority (76\%) of wheelchairs were not secured using four point strap-type tiedowns during transport, and that misuse of the lap belt was common ( $44 \%$ of cases). We reported that misuse of the lap belt often consisted of the LATV operators attempting to secure the wheelchair by routing the lap belt around the wheelchair seatback in an attempt to secure the wheelchair. Wolf, et al. also observed WTORS use on LATVs, ${ }^{15}$ and reported incomplete utilization of available securement systems in $62 \%$ of observations, and no observations of occupant restraint use.

This study presents a comprehensive review of video-recorded, wheelchairrelated adverse events involving disuse and misuse of WTORS during transit, identifying WTORS configurations associated with adverse wheelchair and passenger outcomes. 
Three adverse events involving severe wheelchair and/or wheelchair-seated passenger instability while traveling in an LATV were examined in greater detail.

\section{Methods}

Approval to conduct this study was obtained from the University of Louisville Institutional Review Board (IRB No. 170.07).

In a separate study conducted by Frost and Bertocci, in-vehicle digital video surveillance footage of wheelchair activities in LATVs was reviewed and archived during the twenty-one month period of June 2007 through February $2009^{7}$. Randomly selected digital video recorders containing video footage were provided to the authors by the transit agency. Video footage of wheelchair trips (ingress through egress) were captured and archived for analysis in the Wheelchairs on Fixed-Route Transit (WoFT) database. Individual wheelchair passengers were not identified and so could, theoretically, be represented in multiple trips/events. The WoFT database includes information such as the number of WTORS used, wheelchair type, time required to secure the wheelchair, whether wheelchair related instability occurred during transit, and various other parameters relevant to wheelchair transit. Sixty (60) of the 285 LATVs operated by the transit agency were equipped with the $\mathrm{GE}^{\circledR}$ MobileView III Video Surveillance System ${ }^{\circledR}$ (GE Security, Bradenton, FL). This system records digital video images at a frequency of 30 frames/second with up to 640x480 pixel resolution. Each in-vehicle video surveillance system consists of 4-6 permanently mounted video cameras (Figure 1). Typically, each LATV was equipped with cameras to include views from the front windshield, front and rear of the LATV interior, and front and rear doors, as well as the wheelchair securement 
station. The views of the wheelchair securement station and front of the LATV interior were most often used to observe events for this study. Additional information describing the video collection process is published elsewhere ${ }^{7}$.

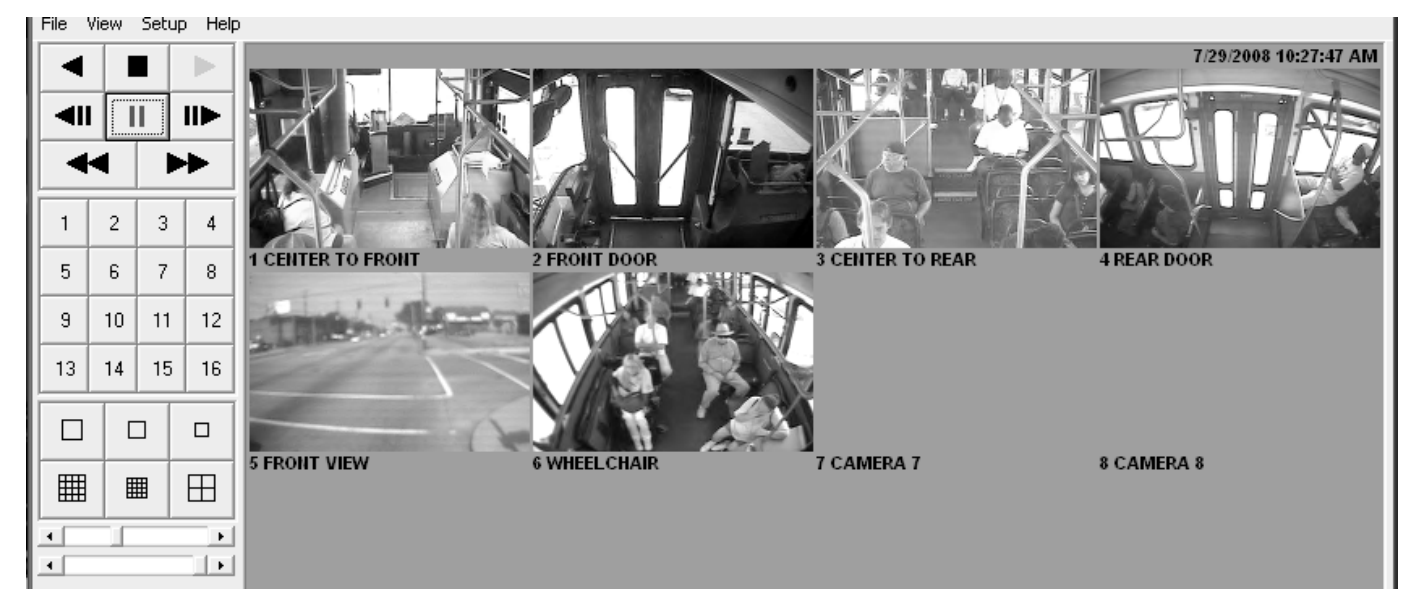

Figure 1. In-vehicle 6-camera LATV video surveillance system. This LATV was equipped with cameras to include views from the front windshield, front and rear of the LATV interior, and front and rear doors, as well as the wheelchair securement station.

The WoFT database contains 295 records. Each record corresponds to a single video file of one wheelchair-seated passenger trip. For this study, records were filtered to extract a subset of adverse events involving wheelchairs and/or wheelchair passengers. An adverse event was defined as any event involving wheelchair and/or wheelchair passenger instability during transit. Because of the low resolution and low frequency of the video data, excursions could not be quantified and the authors qualitatively categorized adverse events into two categories: minor or severe instability. Minor instability was defined by wheelchair excursions when the wheelchair remained within the securement station. Severe instability was defined by wheelchair excursions that exceeded the boundary of the securement station into the aisle and/or wheelchair passenger ejection from their wheelchair. 
Adverse events were also characterized based on wheelchair type, direction of excursion (fore-aft wheelchair excursion, lateral wheelchair excursion, combined fore-aft and lateral wheelchair excursion, and other wheelchair and/or wheelchair passenger excursion) and WTORS configuration (number of tiedowns applied, and whether the lap belt and shoulder belt were used). Two terms were used to categorize occupant restraint usage: disuse was defined as a situation in which neither the shoulder nor lap belt was used, and misuse was defined as use of the lap belt to secure the wheelchair by routing it around the wheelchair seatback.

Cases involving severe instability were reviewed in greater detail, noting responsive actions taken by the wheelchair passenger, LATV operator and/or other passengers. Additionally, dynamics of the wheelchair and/or wheelchair passenger were described along with the dynamics of the other passengers and the LATV driving conditions. Descriptive statistics were used to characterize adverse events (PASW statistical software, Ver. 17 for Mac OS X) based upon assessed variables.

\section{Results}

Wheelchair instability based on wheelchair type

Sixty records (20.3\%) meeting the criteria of an adverse event involving wheelchair and/or wheelchair passenger instability during transit were identified from the WoFT database (Table 1). Out of these 60 adverse events, 57 cases (95\%) were categorized as a minor instability, while three (3) cases (5\%) were identified as having severe instability. The greatest number of wheelchair instability cases involved power wheelchairs ( 31 cases), followed by manual wheelchairs ( 22 cases) and scooters ( 7 
cases). Although a greater number of wheelchair instability cases were experienced by power wheelchairs users, the greatest proportion of wheelchair instability cases, relative to the number of trips for a specific wheelchair type, were experienced by scooter users $(36.8 \% ; 7 / 19)$. For manual wheelchair users, the proportion was $30.1 \%$ (22/73 records), and for power wheelchairs the proportion was $15.3 \%$ (31/202 records).

\begin{tabular}{|c|c|c|c|c|}
\hline & $\begin{array}{c}\text { Total } \\
\text { wheelchair } \\
\text { trips }(\mathrm{n}=295)\end{array}$ & $\begin{array}{c}\text { Proportion of } \\
\text { all wheelchair } \\
\text { trips }\end{array}$ & $\begin{array}{c}\text { Total instability } \\
\text { cases }(\mathrm{n}=60)\end{array}$ & $\begin{array}{c}\text { Proportion of } \\
\text { instability cases } \\
\text { (minor \& severe) }\end{array}$ \\
\hline $\begin{array}{c}\text { Power } \\
\text { wheelchairs }\end{array}$ & 202 & $\begin{array}{c}202 / 295= \\
68.5 \%\end{array}$ & 31 & $\begin{array}{c}31 / 202= \\
15.3 \%\end{array}$ \\
\hline $\begin{array}{c}\text { Manual } \\
\text { wheelchairs }\end{array}$ & 74 & $\begin{array}{c}74 / 295= \\
25.1 \%\end{array}$ & 22 & $\begin{array}{c}22 / 73= \\
30.1 \%\end{array}$ \\
\hline Scooters & 19 & $\begin{array}{c}19 / 295= \\
6.4 \%\end{array}$ & 7 & $\begin{array}{c}7 / 19= \\
36.8 \%\end{array}$ \\
\hline
\end{tabular}

Table 1. Proportion of instability cases by wheelchair type in WoFT database.

\section{Minor wheelchair instability cases}

The tree-diagram in Figure 2 characterizes the direction of excursion and WTORS configuration for each adverse event with minor instability. The second level of the tree diagram indicates the distribution based on direction of excursion. The third level of the tree diagram indicates number of tiedowns used, and the fourth level indicates how occupant restraints were applied. Of the 57 events of minor instability, 25 events (43.9\%) involved a combination of fore/aft and lateral excursions of the wheelchair, while 22 events (38.6\%) involved fore/aft excursions only. Eight events (14.0\%) were associated with lateral excursions only, and 2 events (3.5\%) were categorized as other wheelchair and/or wheelchair passenger instability (e.g. rear wheels of wheelchair lifting off ground, and wheelchair passenger driving wheelchair out of securement station during LATV 
transit). For all subcategories, the most frequent number of wheelchair tiedowns used was zero (79\%) ; twenty of twenty-five events (20 of $25,80 \%$ ) involving fore/aft and lateral excursions, 18 of 22 events (82\%) with fore/aft excursions only, 7 of 8 events (88\%) with lateral excursions, and 2 of 2 events (100\%) with another instability type. Our findings also indicate minimal use of occupant restraints. Occupant restraints were used to restrain the wheelchair-seated passenger in only 14 of 57 adverse events with minor instability (22.8\%). The most frequently observed use of occupant restraints was the misuse of the occupant restraint to secure the wheelchair. Specifically, the lap belt was used to secure the wheelchair by routing the lap belt around the wheelchair seatback. 


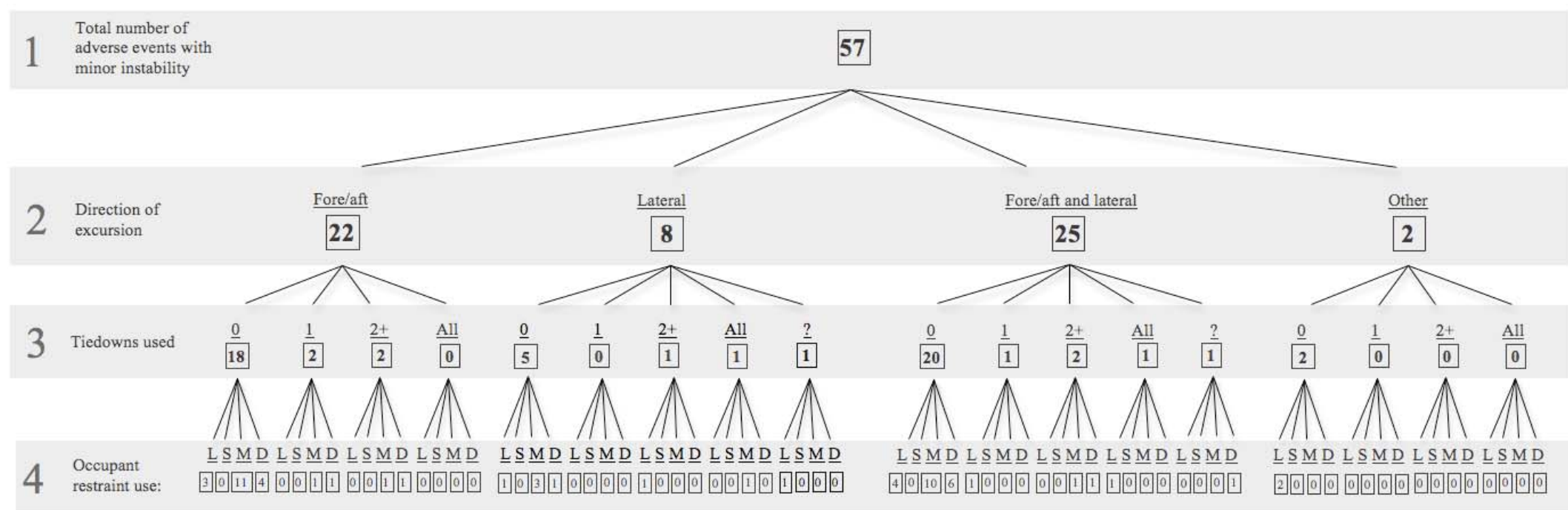

Legend:

$\mathrm{X}$ - X number of cases

$0-$ Zero tiedowns used

$\underline{1}-$ One tiedown used

$2+-$ More than one tiedown used

All - All tiedowns used

$?$ - Unknown number of tiedowns used

\author{
$\underline{L}$ - Lap belt used \\ - Shoulder belt used \\ $\underline{M}$ - Occupant restraint misuse \\ D - Occupant restraint disuse
}

Figure 2. Tree-diagram showing direction of excursion, number of tiedowns used, and occupant restraint use for 57 wheelchair instability events categorized as minor instability. 
We also determined the tiedown and occupant restraint configurations by wheelchair type in all 57 cases of minor instability (Figure 3). 


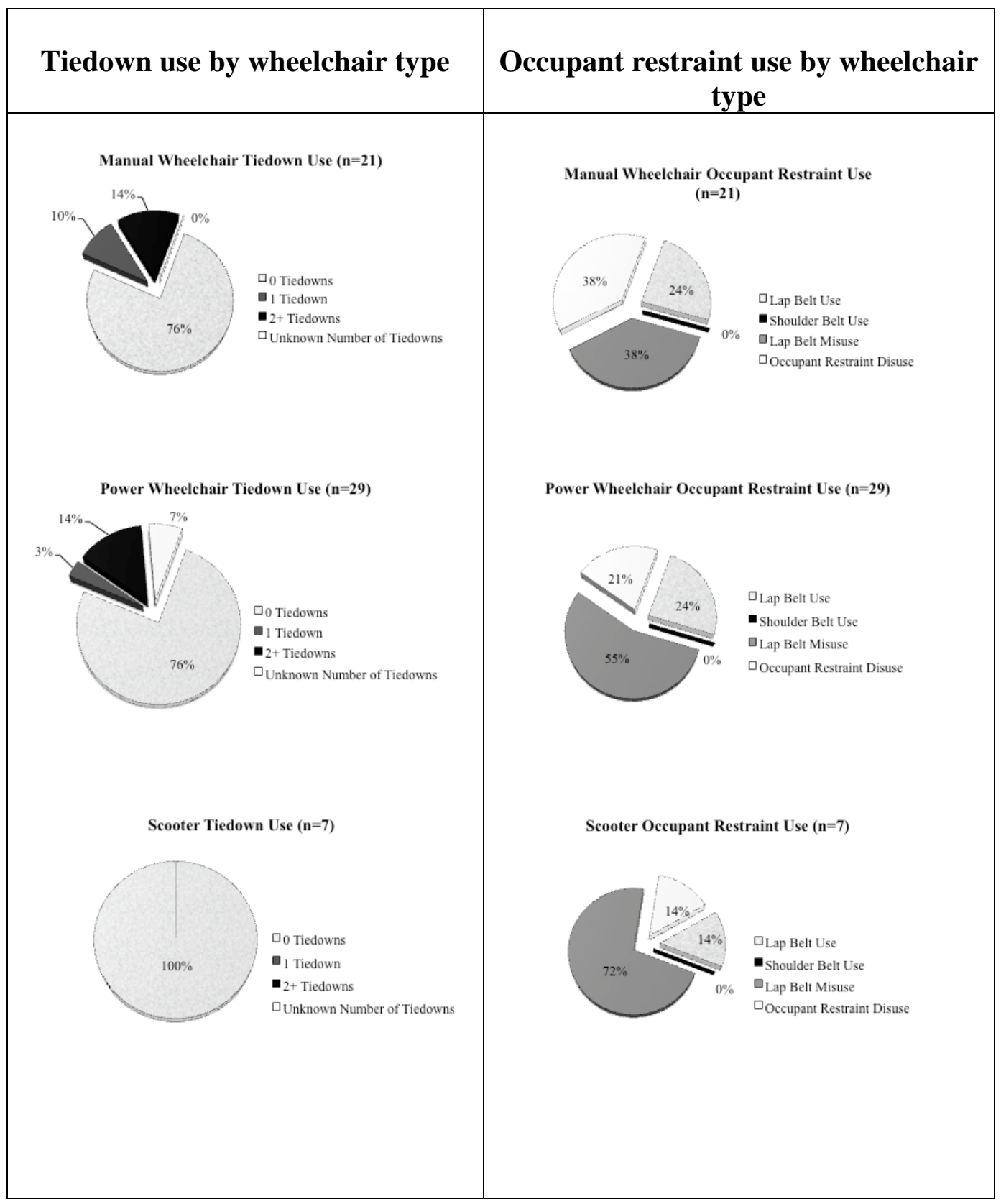

Figure 3. Tiedown and occupant restraint configurations by wheelchair type in minor instability cases. 


\section{Severe wheelchair instability cases}

One manual wheelchair and two power wheelchair passengers experienced an adverse event with severe instability. Summary data for each case is provided in Table 2, followed by a detailed description of each.

\begin{tabular}{|c|c|c|c|c|c|c|c|c|}
\hline \multirow{2}{*}{$\begin{array}{l}\text { Case } \\
\text { No. }\end{array}$} & \multirow{2}{*}{$\begin{array}{c}\text { Wheelchair } \\
\text { type }\end{array}$} & \multirow{2}{*}{$\begin{array}{c}\text { Wheelchair } \\
\text { brakes } \\
\text { applied }^{\dagger} \\
\text { (Yes/No) }\end{array}$} & \multicolumn{4}{|c|}{ WTORS configuration } & \multirow{2}{*}{$\begin{array}{l}\text { LATV } \\
\text { maneuver }\end{array}$} & \multirow{2}{*}{$\begin{array}{l}\text { Instability } \\
\text { Dynamics }\end{array}$} \\
\hline & & & $\begin{array}{c}\text { Tiedowns } \\
\text { used }\end{array}$ & $\begin{array}{c}\text { Lap belt } \\
\text { used } \\
\text { (Yes/No) }\end{array}$ & $\begin{array}{l}\text { Shoulder } \\
\text { belt used } \\
(\text { Yes/No) }\end{array}$ & $\begin{array}{c}\text { ORS } \\
\text { Misuse* } \\
\text { (Yes/No) }\end{array}$ & & \\
\hline 1 & $\begin{array}{c}\text { Power } \\
\text { wheelchair }\end{array}$ & No & 0 & No & No & Yes & $\begin{array}{c}\text { Traveling } \\
\text { straight at } \\
\text { routine speed }\end{array}$ & $\begin{array}{c}\text { Wheelchair } \\
\text { driven into } \\
\text { aisle }\end{array}$ \\
\hline 2 & $\begin{array}{c}\text { Manual } \\
\text { wheelchair }\end{array}$ & Yes & 0 & Yes & No & No & $\begin{array}{l}\text { Routine right } \\
\text { turn }\end{array}$ & $\begin{array}{l}\text { Wheelchair } \\
\text { rotated } \\
\text { laterally into } \\
\text { aisle }\end{array}$ \\
\hline 3 & $\begin{array}{c}\text { Power } \\
\text { wheelchair }\end{array}$ & Yes & 0 & No & No & No & $\begin{array}{l}\text { Routine } \\
\text { braking }\end{array}$ & $\begin{array}{l}\text { Wheelchair } \\
\text { passenger } \\
\text { ejected from } \\
\text { wheelchair }\end{array}$ \\
\hline
\end{tabular}

* - Lap belt routed around wheelchair seatback to secure wheelchair

$\dagger$ - When a power wheelchair is turned off, the transmission becomes disengaged, serving as the braking mechanism. In case 1 , the power wheelchair was not turned off and the brakes were therefore not applied.

Table 2. Summary data for severe instability cases

\section{Case 1: Power wheelchair driven into aisle}

A male wheelchair passenger boarded the LATV and positioned his mid-wheeldrive power wheelchair forward facing in the entry-door-side (LATV direction of travel) securement station. An augmentative communication device was mounted to the wheelchair at the passenger's mid-torso level. Power to the wheelchair was not turned off prior to transit. The operator did not apply any tiedowns to secure the wheelchair, nor were the occupant lap and shoulder belts applied to restrain the passenger. The operator 
instead misused the lap belt by loosely wrapping it around the wheelchair seatback in an apparent attempt to secure the wheelchair.

The LATV appeared to be traveling at a constant forward velocity, and was not turning, accelerating or braking. Approximately one minute into transit, the power wheelchair appeared to rotate laterally into the aisle space (Figures 4 and 5). The wheelchair passenger remained seated in the wheelchair during this excursion. No ambulatory passengers were standing in the aisle during transit and no passengers were injured. An ambulatory passenger sitting across the aisle from the wheelchair passenger moved his right foot in an apparent attempt to avoid injury, and then used his right foot to restrain the left front wheelchair footrest to prevent the wheelchair from moving farther into the aisle.

Video review revealed hand movement near the wheelchair controller immediately preceding wheelchair excursion. It appeared that the wheelchair passenger engaged the controller, causing the wheelchair to rotate into the aisle space. The LATV operator stopped the vehicle en route and manually repositioned the power wheelchair in the securement station. The operator spoke with the ambulatory passenger and then appeared to turn the power off to the wheelchair. The operator again did not secure the wheelchair using tiedowns, nor did she apply the shoulder belt or tighten the lap belt, which was still loosely wrapped around the seatback. No further instability occurred during the remainder of the trip. 


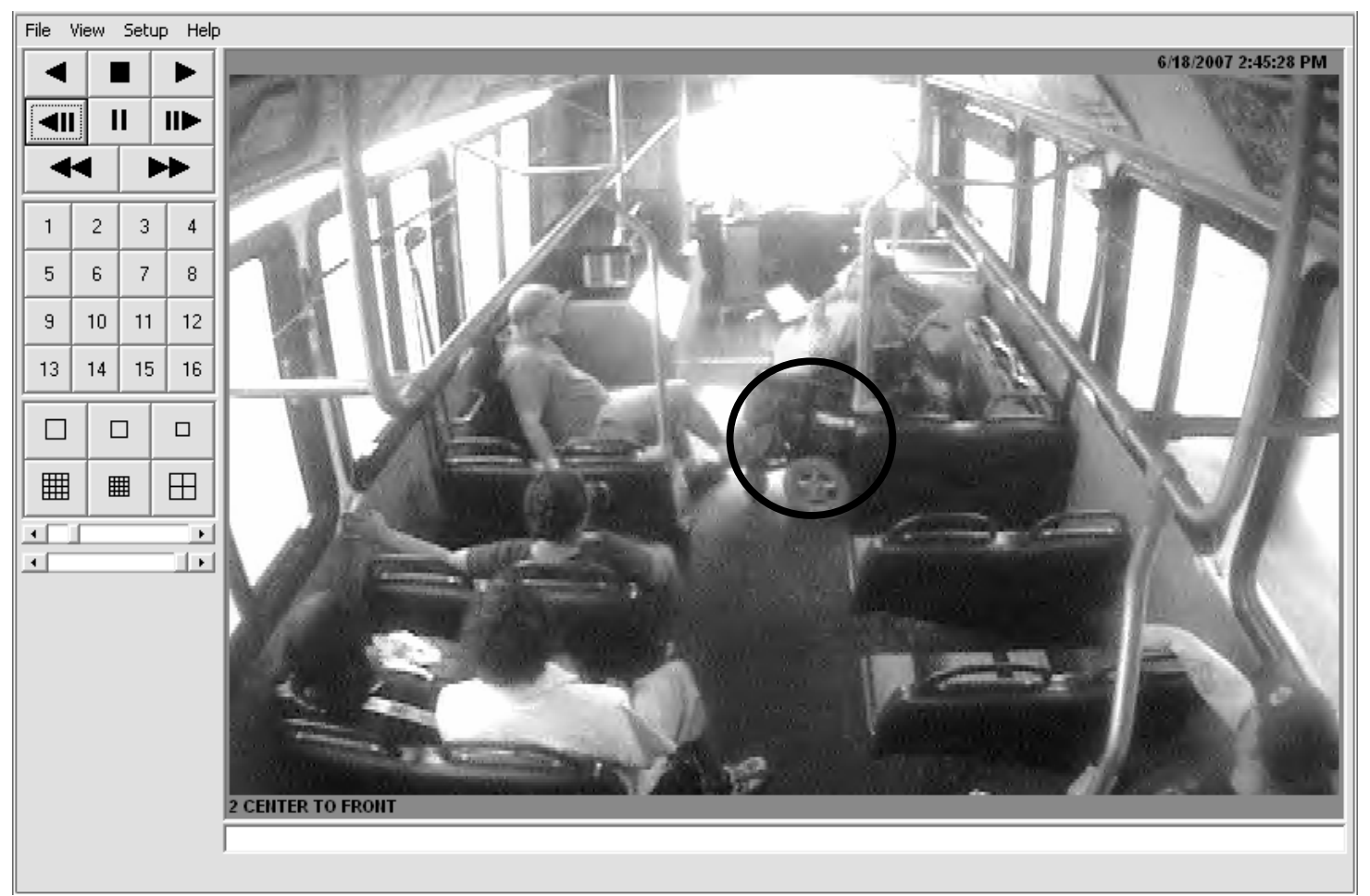

Figure 4. Case 1: Unsecured power wheelchair enters aisle during routine LATV transit, while sitting ambulatory passenger lifts foot to prevent injury and limit power wheelchair movement. (View from LATV rear).

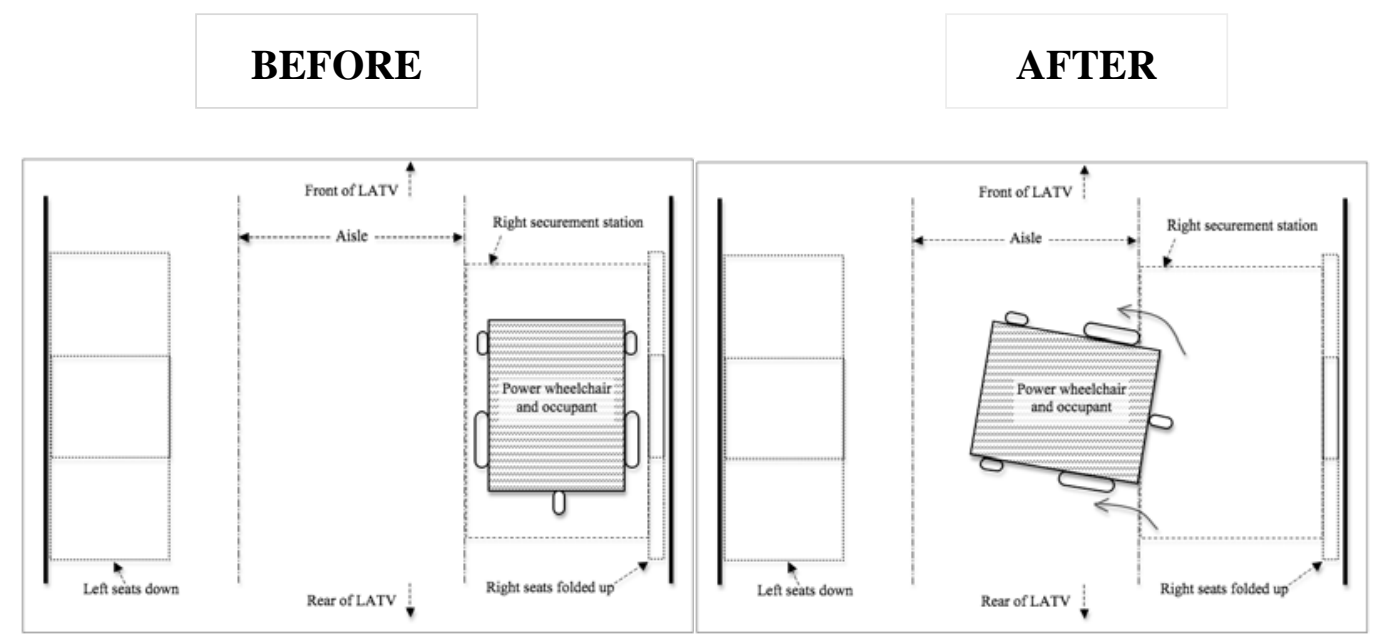

Figure 5. Diagram showing unsecured power wheelchair rotating laterally into aisle during routine LATV transit (Case 1). 


\section{Case 2: Manual wheelchair rotated laterally into aisle}

A male wheelchair passenger boarded the LATV, positioned his manual wheelchair forward-facing in the entry-door side securement station and applied both brakes. The LATV operator did not apply any tiedowns to secure the wheelchair. To restrain the wheelchair passenger, the operator applied the lap belt correctly under the wheelchair armrests, albeit very loosely across the wheelchair passenger's pelvic region. The shoulder belt was not used.

Two minutes into transit, the LATV initiated a right turn. During the turn, the passenger and wheelchair rotated laterally into the aisle (Figures 6 and 7). As the wheelchair rotated into the aisle, the passenger was observed grabbing the folding-seat under the window with his right arm, and stabilizing himself by placing both feet on the ground. An ambulatory passenger sitting across the aisle prevented additional excursion of the wheelchair into the aisle by extending her right leg and using her foot to push against the left rear wheel of the wheelchair. There were no passengers standing in the aisle and no passengers were injured. Immediately after the adverse event occurred, as the LATV came to a stop, the wheelchair passenger repositioned his wheelchair in the securement station. Once the vehicle was stopped, the LATV operator returned to the securement station and secured the wheelchair using all four tiedowns; he did not apply the shoulder belt or reapply the lap belt. No further instability occurred during the remainder of the trip. 


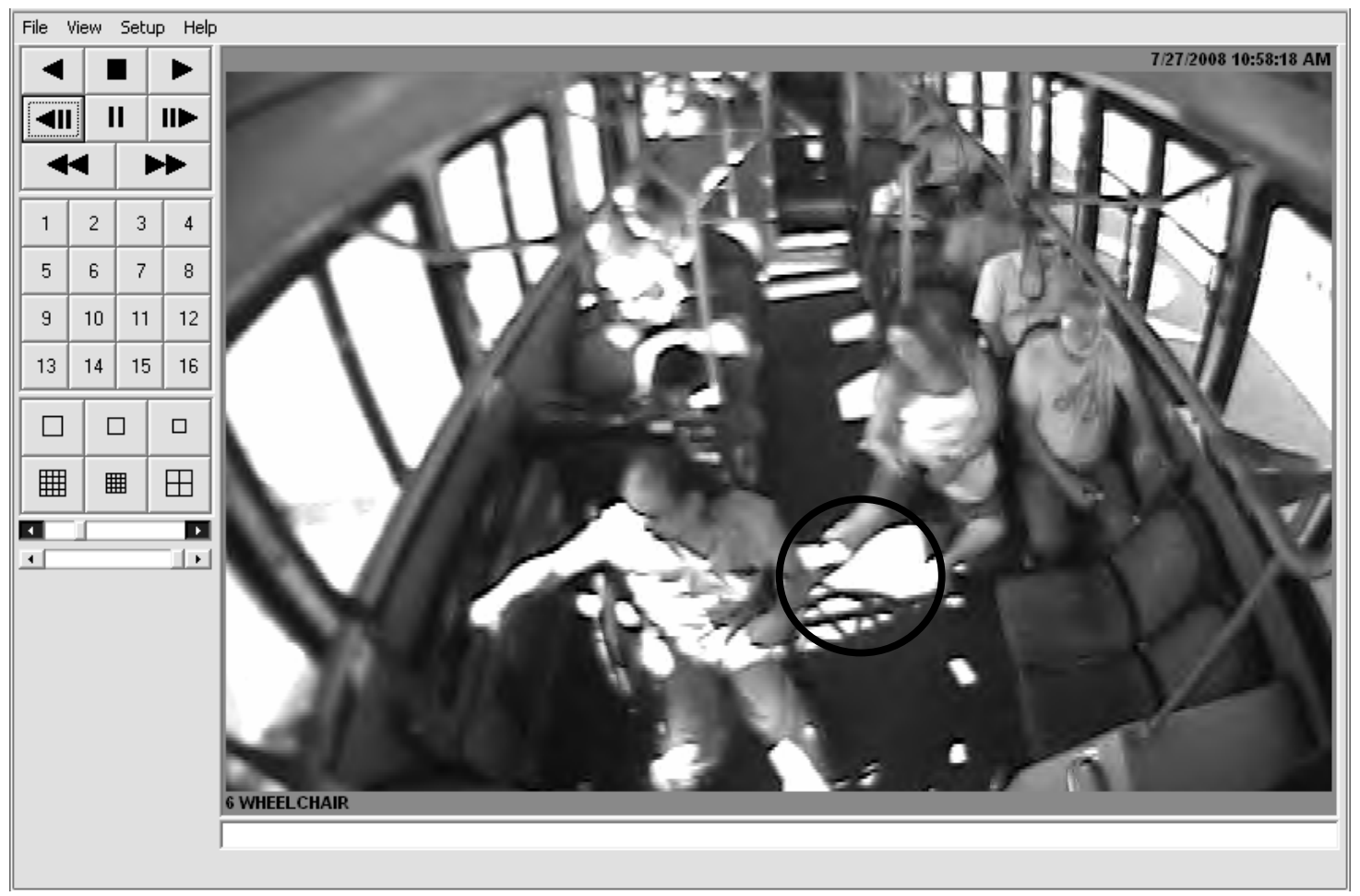

Figure 6. Case 2: Manual wheelchair passenger and wheelchair rotated into aisle as LATV performed a right turn. Wheelchair passenger stabilized himself by placing both feet on the floor and using his right arm to grab the folding-seat while an ambulatory passenger used her foot to prevent additional lateral excursion of the wheelchair. (View from LATV front).

\section{BEFORE}

AFTER

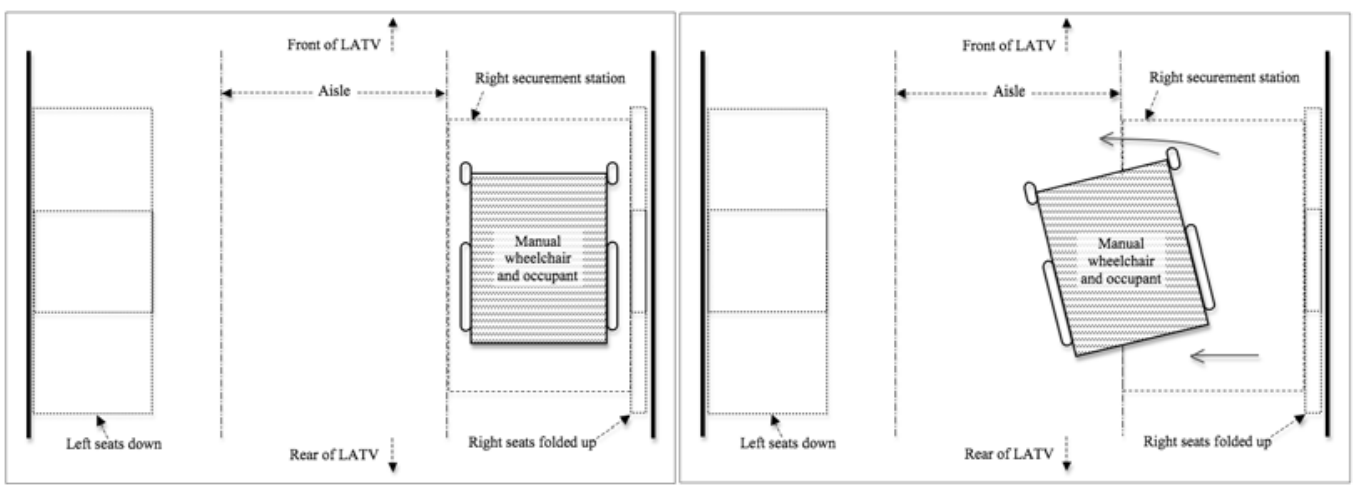

Figure 7. Diagram showing manual wheelchair and wheelchair passenger rotating laterally into the aisle during a routine right turn. Note: orientation of this diagram is $180^{\circ}$ opposite of Figure 6 camera view. 


\section{Case 3: Power wheelchair passenger ejected from wheelchair}

A female wheelchair passenger boarded the LATV and positioned her power wheelchair forward-facing in the entry-door side securement station. The power wheelchair was equipped with captain-style or standard upholstered seating that appeared inappropriately sized (too small) for the bariatric wheelchair passenger. The wheelchair passenger appeared to turn off power to the wheelchair. The LATV operator did not secure the wheelchair with any tiedowns and did not apply the lap or shoulder belts to restrain the occupant.

The LATV was traveling in a forward direction at apparent routine speed relative to traffic. As the LATV approached an intersection, the vehicle appeared to slow down via normal braking, and the wheelchair passenger was ejected from her wheelchair into the aisle. Video observation revealed that the wheelchair remained in position within the securement station, as the LATV reduced speed. The wheelchair passenger was ejected in a forward direction from her wheelchair (Figures 8 and 9). During ejection, the wheelchair passenger attempted to stabilize herself (stop her forward motion) by using her right arm to prevent impact with the LATV bulkhead directly in front of the securement station. The passenger struck the bulkhead with her lower extremities at knee level and fell laterally to the aisle floor. An ambulatory passenger sitting across the aisle attempted to restrain the wheelchair passenger with an outstretched arm during her ejection and fall. There were no passengers standing in the aisle, nor were any other passengers injured.

Immediately after the adverse event occurred, the LATV came to a stop. The wheelchair passenger could be heard on the video complaining that her leg was fractured. 
Four ambulatory passengers, and later emergency medical services personnel failed in an attempt to lift the injured wheelchair passenger back into her wheelchair. The wheelchair passenger crawled to the front door of the LATV, where she was placed on a stretcher and transported to an ambulance.

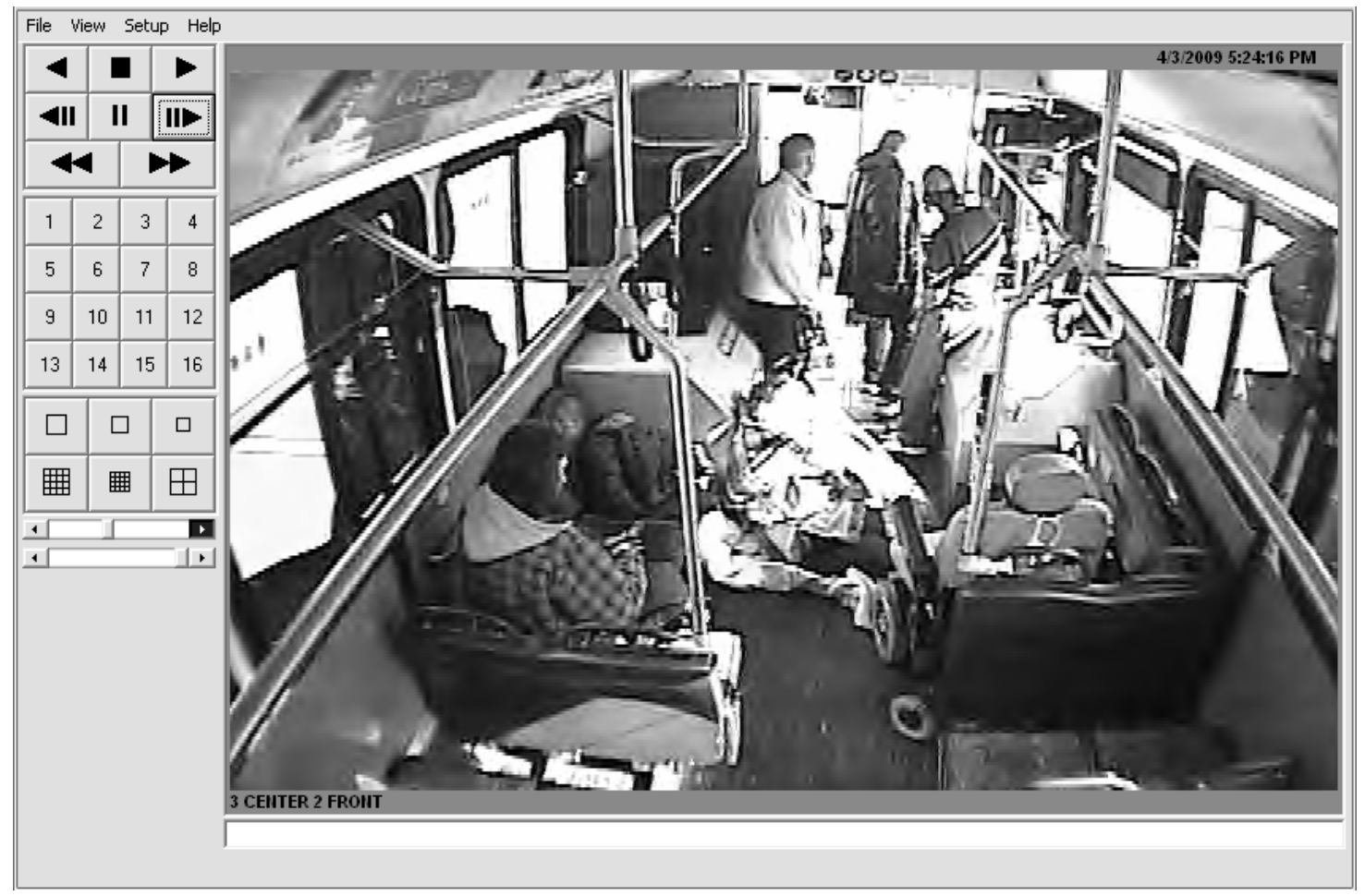

Figure 8. Case 3: Power wheelchair passenger ejected from wheelchair during normal LATV braking. Wheelchair passenger struck the bulkhead in front of the securement station and fell to the floor complaining of a fractured leg. (View from LATV rear). 


\section{BEFORE}

AFTER

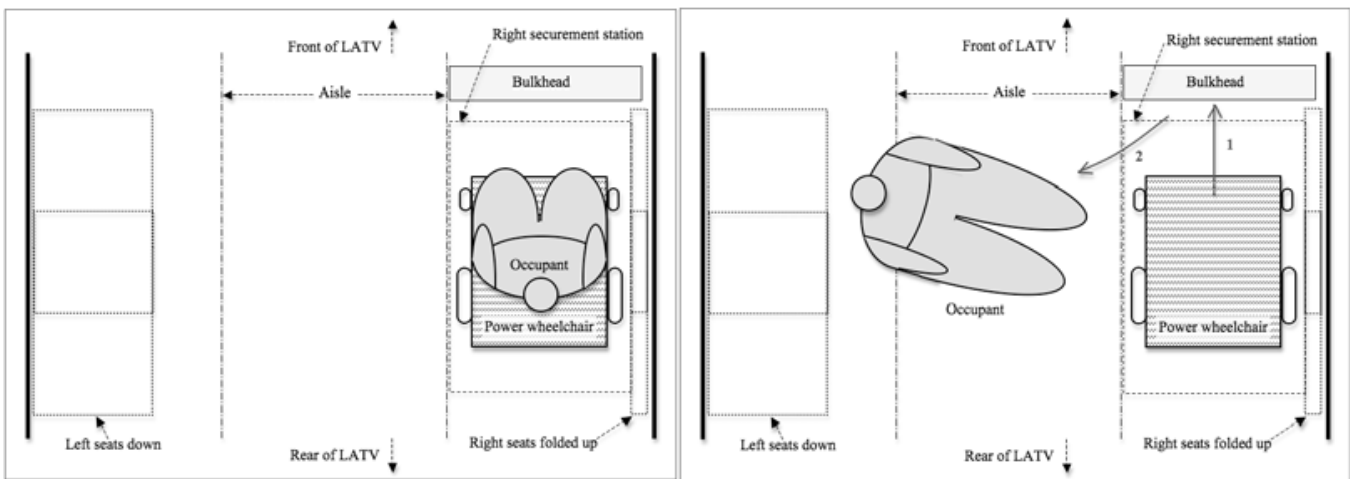

Figure 9. Diagram showing power wheelchair passenger ejected from power wheelchair during an apparent routine braking maneuver. During ejection, (1) the wheelchair passenger struck the LATV bulkhead directly in front of the right securement station, and then (2) fell to the floor in the aisle.

\section{Discussion and Conclusions}

To our knowledge, this is the first observational study to examine wheelchair instability in LATVs based on in-vehicle video footage of actual events. Our objective was to characterize adverse events involving wheelchair instability related to WTORS disuse and misuse onboard LATVS.

The overall relatively high occurrence (20.3\%) of adverse events (wheelchair related instabilities) found in this study could potentially be lowered if the frequency of proper WTORS use is increased. This may be achieved through additional training of the LATV operators by transit agencies and through increased wheelchair passenger awareness as to the risks associated with improper WTORS use. 


\section{Wheelchair instability based on wheelchair type}

We found that power wheelchair users represented the greatest percentage of wheelchair passengers in fixed-route transit $(68.5 \%)$, followed by manual wheelchair users $(24.7 \%)$, and scooter users (6.4\%). This finding is consistent with data published by Buning, et al. ${ }^{10}$. Buning, et al. surveyed public transit wheelchair passengers $(\mathrm{n}=283)$ nationally and found that $63.3 \%$ used power wheelchairs, $27.9 \%$ used manual wheelchairs, and $5.9 \%$ used power scooters.

The results from this study also show that despite the greater percentage of power wheelchairs used as seats in LATVs, scooter and manual wheelchairs exhibit greater instability during transit (36.8 \% and 30.1\%, respectively vs. $15.3 \%$ for power wheelchairs). Although the sample size may be limited, these findings may suggest a pattern of wheelchair instability by wheelchair type. All scooters were three-wheeled designs, however there was no specific pattern in the direction of excursion for scooter instability cases. Scooters have been found to possess a lower center of gravity (CG) in comparison to power and manual wheelchairs ${ }^{16}$, a characteristic that generally increases stability. There may also be structural features (e.g. three-wheeled designs) that contribute to scooter instability as well as inaccessible frames that generally do not allow for easy securement using four-point, strap type tiedowns. Scooters may have been less likely to be secured properly given housings that typically enclosed their structural frames. Design differences between manual and power wheelchairs may also contribute to differences in wheelchair stability during LATV transit. Compared to power wheelchairs, manual wheelchairs have smaller mass and lower inertia, higher $\mathrm{CG}^{16}$, and generally less robust brake hardware. When a power wheelchair is turned off, the 
transmission becomes disengaged, acting as a brake. Manual wheelchair brakes can become misaligned with the rear wheels, making the braking mechanism less effective. Additionally, manual wheelchair brakes are operated by hand and require the application of force to the brake mechanism lever to engage the brake, a task that may be challenging for some manual wheelchair users.

\section{Cases with minor instability}

Tiedown usage patterns may be directly related to wheelchair instability. In this study we found that zero (0) was the most frequent number of tiedowns used for each type of instability and in only 2 out of 57 cases (3.5\%) were all four tiedowns used to secure the wheelchair (tree-diagram, Figure 2). However, given our reliance on video footage it is not known if tiedowns were applied correctly, using the appropriate securement point on the wheelchair and properly tensioning the tiedowns when used; both could substantially affect the tiedowns' ability to maintain wheelchair securement during LATV transit.

We also found that the most prevalent occupant restraint system (ORS) configuration associated with zero tiedowns across all instability types was the misuse of the lap belt in an attempt to secure the wheelchair. Previously reported by Frost and Bertocci ${ }^{7}$, this type of ORS misuse consisted of the lap belt routed around the wheelchair seatback with no use of the shoulder belt. The lap belt tension during such ORS misuse was indeterminable. Since the most frequent number of tiedowns used was zero and misuse of the lap belt was most the prevalent ORS configuration observed for all instability types, it may be concluded that the LATV operators observed in this study are 
aware of the need to provide wheelchair securement, but are uncertain or are unable to apply WTORS properly. LATV operators may also believe that this short cut method is good enough to provide adequate transportation safety.

The WTORS policy of the transit agency in study is to require tiedown use, but leave ORS use at the discretion of wheelchair passengers. An improved understanding as to why operators are not properly implementing WTORS is needed. Currently, LATV operator training in the proper use of WTORS includes classroom instruction and demonstration, as well as supervised hands-on practice performed in an LATV. Perhaps, additional and improved in-depth operator training on proper WTORS implementation could be helpful in avoiding future misuse and disuse of WTORS. LATV operator training could also feature examples of adverse outcomes associated with WTORS disuse and misuse. Wheelchair passengers' awareness of and eagerness to request proper WTORS use can also be important in reducing adverse events.

\section{Cases with severe instability}

\section{Case 1: Power wheelchair driven into aisle}

Turning off the power to the wheelchair prior to transit may have prevented the power wheelchair from rotating into the aisle, even if the wheelchair passenger inadvertently struck the control joystick. Also, had all four tiedowns been applied properly (appropriate securement point locations with proper tiedown tension), the power wheelchair would likely not have been able to enter the aisle space.

Although no passengers were injured, the outcome may have been different if passengers were standing in the aisle during transit. This raises the point that proper 
WTORS application can not only increase transportation safety for wheelchair passengers, but also increase safe transit for general ridership. Additionally, had the passenger sitting across the aisle from the securement station not attempted to prevent additional wheelchair excursion into the aisle, injuries to both individuals may have occurred.

\section{Case 2: Manual wheelchair rotated laterally into aisle}

As the manual wheelchair and wheelchair passenger rotated into the aisle during a routine LATV right turn, the passenger's ability to restrain himself using his extremities appeared to limit additional excursion into the aisle. Other wheelchair passengers may not have the same level of voluntary muscle control or the ability (strength) to restrain themselves as effectively, placing them at an increased risk for injury. Had the wheelchair been properly secured with tiedowns, the lateral wheelchair rotation into the aisle may have been prevented.

\section{Case 3: Power wheelchair passenger ejected from wheelchair}

The power wheelchair passenger appeared to be using an undersized power wheelchair, equipped with captain seating. This type of seating system in general is not easily adjustable and is typically not designed for persons who may have special seating needs, such as bariatric wheelchair users. This illustrates that wheelchair passenger safety on LATVs may not be limited to the issue of proper WTORS application, as proper wheelchair prescription (through involvement of qualified therapist) is also important in wheelchair passenger safety. The use of properly applied occupant restraints (lap and/or 
shoulder belt) would likely have prevented the wheelchair passenger's ejection from her power wheelchair. Finally, without the restraining assistance provided by the ambulatory passenger, the wheelchair passenger may have incurred additional injuries.

\section{$\underline{\text { Summary of cases with severe instability }}$}

It is important to note that all three cases of severe instability occurred during normal driving maneuvers. This finding adds to an emerging body of evidence indicating that crash conditions need not be present to subject wheelchair passengers to increased risks while traveling in LATVs. According to a study by Frost and Bertocci ${ }^{7}$, of the wheelchair-related incidents on LATVs that took place when the LATV was moving, $76.7 \%$ occurred under normal driving conditions compared to $23.3 \%$ of incidents that occurred during emergency maneuvers. Wretstrand, et al. ${ }^{12}$ also found similar results, reporting that most wheelchair user injuries occurred during routine driving maneuvers of para-transit vehicles.

Under the broad non-discrimination provisions in the ADA regulations, transit providers must provide tiedowns, but may choose whether or not to mandate their use. However, transit providers may not mandate the use of lap and shoulder belts by wheelchair users unless they mandate this use by all passengers ${ }^{8}$. As a result, wide disparities amongst transit agency WTORS policies have been documented ${ }^{9,10}$. Buning, et al. ${ }^{10}$ found that while the majority $(66.7 \%)$ of transit providers surveyed required tiedown use for transporting wheelchair passengers, only $25.6 \%$ of those providers also required occupant restraints, and 69.5\% made occupant restraints optional. Almost eighteen percent (17.9\%) of transit providers reported optional wheelchair securement, 
and out of those, $90.9 \%$ have policies with optional occupant restraints for wheelchair passengers. The findings from our study suggest that proper use of WTORS on LATVs may reduce the number of wheelchair-related adverse events. Increasing the uniformity of transit agency WTORS policies may also increase proper use of WTORS, thus reducing wheelchair related adverse events on LATVs.

Regarding use of four-point, strap-type WTORS on LATVs, confusion is also evident on the part of wheelchair passengers ${ }^{9,10}$. With regard to requesting securement, Buning, et al. ${ }^{10}$ found that $39 \%$ of wheelchair passengers never asked to be secured compared with $36.6 \%$ who routinely asked to be secured. Increased awareness regarding the importance of proper WTORS use on the part of wheelchair passengers may lead to increased compliance with transit agency policies regarding WTORS usage. Additionally this might encourage some wheelchair passengers to play a more active role in responsibility for their safety by requesting WTORS and directing their proper use.

As indicated herein, challenges exist with the use of four-point strap-type WTORS to secure wheelchair passengers on LATVs. Thus alternative strategies for wheelchair securement or retention such as auto-docking systems and rear-facing wheelchair passenger stations are being explored ${ }^{17,18}$. Auto-docking systems use hardware that interfaces the wheelchair directly to the docking system, which is installed in the LATV. Rear-facing wheelchair passenger stations require passengers to back their wheelchair against a vehicle-mounted, padded restraint that fits in close proximity to the passenger's head and back to prevent against forward wheelchair excursions. A vertical stanchion may be present, or a horizontal side barrier may fold down to prevent lateral wheelchair excursion. These alternative systems may increase wheelchair retention or 
securement, since they can be used independently or with minimal LATV operator

involvement. National and international standards (SAE J2249 ${ }^{14}$, ISO $10542{ }^{19}$, and ISO

$10865-1^{20}$ ) addressing these systems allow for design flexibility, providing an opportunity for alternative means to safely secure wheelchairs and restrain wheelchair passengers. While these alternatives provide benefits, there are drawbacks, such as the need for additional hardware on wheelchairs to use the auto-docking system (affecting ground clearance, weight, and in some cases overall wheelchair dimensions ${ }^{18}$ ), and difficulties associated with facing rearward during transit. Additionally, the safety of rear-facing passenger systems has yet to be thoroughly investigated.

\section{$\underline{\text { Limitations }}$}

The WoFT data is based on video surveillance footage from only one transit agency from a major metropolitan area in a mid-western geographical area. Wheelchairrelated data from other transit agencies in other regions of the country may produce different results. The video data reviewed for this study was of relatively low resolution (640x480 pixels) and low frequency $(30 \mathrm{~Hz})$. Because of low frequency and lowresolution video, the categorization of minor and severe instability was somewhat subjective; others may have included/excluded different events. Higher resolution and higher frequency video data may provide improved details including quantifiable wheelchair and/or passenger excursions. Since LATVs were equipped with varying numbers of cameras (4-6), limited view angles were available in some cases. In these cases, additional cameras may have provided more detailed information. Our assessment of video footage only determined whether WTORS were used, but did not allow us to 
determine WTORS tension/angles and the exact location of securement on wheelchair. Additionally, the transit agency's practices include random assignment of LATVs to routes on a daily basis; and the majority of LATV operators are also randomly assigned routes for each work shift. Given these practices, and the relatively low resolution of the video images, we made no effort to identify or filter videos for wheelchair passengers or LATV operators who may have been recorded more than once. As a result, it is possible that the data contain multiple trips of one or more wheelchair passengers, and multiple trips involving one or more LATV operators. Finally, this study did not include testimonies from involved parties; the LATV operator, wheelchair passenger, and other passengers may have been able to provide supplementary information to aid in better understanding the underlying causes of the adverse events.

\section{$\underline{\text { Conclusions }}$}

The identified adverse events in this study occurred during normal driving, and the vast majority involved minor instability and only a small number were severe instability cases. When normalized by the number of trips for each wheelchair type, scooters had the highest proportion of instability, followed by manual wheelchairs, and power wheelchairs.

The most common direction of wheelchair excursion was a combination of fore/aft and lateral excursions; followed by fore/aft excursion only, lateral excursion only, and other excursion types. For adverse events, the most common number of wheelchair tiedowns used was zero, while the most common occupant restraint 
configuration was the misuse of the lap belt to attempt to secure the wheelchair and not to restrain the wheelchair passenger.

The three cases of severe instability suggested that wheelchair-related adverse events could occur during normal LATV driving maneuvers. Applying wheelchair brakes or turning off power wheelchairs, using all four tiedowns correctly, and using the lap and shoulder belt properly may have prevented these adverse events. Ambulatory passengers may also be at increased risk of injury if a wheelchair related adverse event occurs during LATV transit. 


\title{
CHAPTER 3
}

\section{INFLUENCE OF TRANSIT BUS NORMAL DRIVING CONDITIONS ON WHEELCHAIR OCCUPANTS AND WTORS LOADS}

\begin{abstract}
Overview
Large accessible transit vehicles (LATVs, large city transit buses) are a common mode of transportation for wheelchair users, many of whom remain seated in their wheelchairs during transit. The purpose of this study was to gain an understanding of the kinetic environment experienced by a wheelchair-seated passenger onboard an LATV under normal driving conditions. The wheelchair passenger was represented by an anthropomorphic testing device (ATD) seated in manual transit wheelchair and was restrained using a lap and shoulder belt. The wheelchair was secured with 4-point, straptype tiedowns. During intra-city, normal driving the LATV underwent 87 acceleration, 81 braking, 10 left turn, and 10 right turn maneuvers. The LATV acceleration, ATD head, chest and pelvis acceleration, lap and shoulder belt and tiedown loads were recorded for all maneuvers. Results revealed that mean peak LATV acceleration was highest for right turns $(0.37 \mathrm{~g})$, likely because of a relatively small turning radius associated with a right turn maneuver. Across all maneuvers, the mean peak tiedown load was less than $12 \mathrm{lb}$ while mean peak occupant restraint load was less than $3 \mathrm{lb}$. WTORS loads were
\end{abstract}


substantially lower than those reported in other high-g (crash environment) studies. Finally, our study provided data, which may advance alternative approaches to wheelchair securement/retention and passenger restraint.

\section{Introduction}

Large accessible transit vehicles (LATVs, large city transit buses) are generally considered a safe mode of transportation with a low fatality incidence of all passengers ${ }^{1}$. This is due in part to overall vehicle mass and the slow speed of LATV travel associated with intra-city routes. Despite this relatively safe environment, Shaw and Gillispie ${ }^{3}$ found that wheelchair seated passengers have an accident rate over 350 times greater than that of passengers seated in original equipment manufacturer vehicle seats. The U.S. National Highway Transportation Safety Administration (NHTSA) reported that 35\% of injuries and deaths that occurred between 1990-1995 involving wheelchair-seated passengers or drivers were due to improper or lack of securement of the wheelchair, and $17 \%$ of these incidents occurred in LATVs ${ }^{4}$.

The vast majority of surveyed wheelchair passengers $(85 \%)$ remain seated in their wheelchair during transit ${ }^{10}$, which may put them at increased risk of injury if their wheelchairs are not properly secured. Songer, et al. (2004) found that passengers using both public and private transit, who remained seated in their wheelchair during transit experienced higher injury rates (5.2 passenger injuries per 100,000 miles) than those who transferred to a motor vehicle seat (0.6 passenger injuries per 100,000 miles) ${ }^{6}$. In a study on wheelchair related incidents specific to LATV transit, Frost and Bertocci ${ }^{7}$ found that $20 \%$ of all incidents occurred during normal driving. Certainly the proper usage of 
wheelchair tiedown and occupant restraint systems (WTORS) would be expected to provide safe transit under normal LATV driving conditions.

It is necessary to afford wheelchair passengers the same level of transportation safety as the general population. The Americans with Disabilities Act (ADA) ${ }^{13}$ requires that LATVs be equipped with a wheelchair securement device and lap and shoulder belts. A common method to secure the wheelchair and restrain the wheelchair occupant is with the use of four-point, strap type WTORS, consisting of front left tiedown (FLTD), front right tiedown (FRTD), rear left tiedown (RLTD), rear right tiedown (RRTD), a lap belt (LB), and a shoulder belt (SB). Although the ADA requires the provision of a wheelchair securement device and occupant restraints (lap and shoulder belts), it does not mandate the use of such systems ${ }^{8}$. Individual transit agencies are left to establish policies requiring the use of wheelchair tiedowns. Additionally, the ADA does not require mandatory use of lap and/or shoulder belt restraints for wheelchair-seated passengers unless the same mandate has been made for the general ridership ${ }^{8}$. As a result, there are disparities across transit agency policies with respect to WTORS use ${ }^{10}$.

Disuse and misuse of WTORS have been shown to place wheelchair-seated passengers at greater risk of injury. Shaw and Gillispie ${ }^{3}$ identified misuse as a factor contributing to wheelchair passengers injuries in mass transit. In our previous study ${ }^{21}$, we reviewed video surveillance records of WTORS usage on LATVs and found that the majority (76\%) of wheelchairs were not secured using four point strap-type tiedowns during transport, and that misuse of the lap belt was common ( $44 \%$ of cases). Misuse of the lap belt often consisted of the LATV operators attempting to secure the wheelchair by 
routing the lap belt around the wheelchair seatback. The lap belt was used to restrain the wheelchair passenger in only $20 \%$ of observed cases.

Because of reported disuse and misuse of strap-type, four-point WTORS in the LATV low-g environment, alternative solutions to wheelchair securement or retention such as auto-docking ${ }^{18}$ and front-facing ${ }^{22}$ and rear-facing ${ }^{17}$ wheelchair passenger stations are being investigated. A study by Zaworski, et al. ${ }^{11}$ has described in part the low-g LATV environment (LATV accelerations for various normal and emergency driving maneuvers). Another study by Wolf, et al. ${ }^{15}$ used an unvalidated computer simulation model to investigate effects of WTORS configuration on wheelchair passenger safety during normal LATV maneuvers. Previous studies by Sprigle and Linden ${ }^{23,24}$ focused on describing the dynamic environment wheelchair passengers encounter while traveling in a van (reporting vehicle and wheelchair passenger accelerations). Kamper, et al. ${ }^{25-27}$ developed a technique and portable system to assess postural response of wheelchair passengers in vehicles. However, the authors are not aware of any studies to-date that experimentally describe the kinetic environment that a manual wheelchair passenger experiences during normal driving. By providing WTORS loading, and ATD accelerations, in addition to LATV accelerations, our study provides additional data that may advance alternative WTORS methods in the LATV environment.

\section{Methods}

This experimental study focused on describing the kinetic environment experienced by a surrogate wheelchair passenger along with WTORS loading during normal LATV driving. The LATV (Gillig, Year: 2010, Model \# G30D102N4) used in the 
experimental evaluation was a hybrid, $40 \mathrm{ft}(12.2 \mathrm{~m})$, low floor, pneumatic suspension, fixed-route, intra-city bus. The LATV was one of 285 large, public transit buses in the transit agency's fleet. The route used for testing ("Medical Route Circulator," Figure 1) connects various medical facilities in a medium-sized metropolitan city; a relatively level-terrained ( $461 \pm 9 \mathrm{ft}, 141 \pm 3 \mathrm{~m}$ above sea level), urban setting with stop-and-go, inner-city traffic. This route was selected because of a high prevalence of wheelchair passengers.

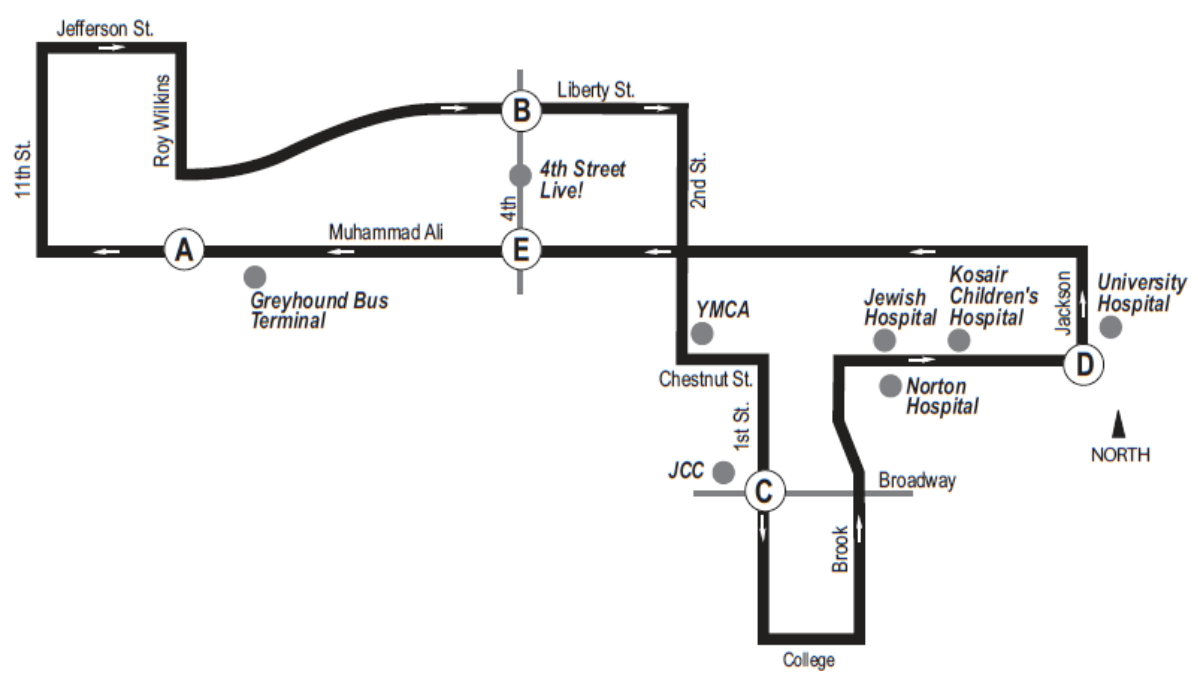

Figure 1. LATV route driven during testing.

The complete route was driven two times and included a total of 87 acceleration (from standstill), 81 braking (to standstill), 10 left turn, and 10 right turn maneuvers. Testing was performed in dry weather conditions and excluding the research group, no passengers were on board the LATV during testing. The same licensed LATV operator was used during all testing and was instructed to drive as if the LATV were in service (making all stops, obeying standard traffic laws, kneeling the LATV if necessary, etc.) 
The wheelchair passenger was represented by a wheelchair-seated, $50^{\text {th }}$ percentile Hybrid II anthropomorphic testing device (ATD - $172 \mathrm{lb}, 78.2 \mathrm{~kg}$ ), while the wheelchair (Quickie 2, Sunrise Medical) used during testing was an ultra-light, X-braced, manual wheelchair with the transit option (conforming to the ISO 7176-19 standard "Wheeled mobility devices for use as seats in motor vehicles" ${ }^{28}$ ). The wheelchair was adjusted to properly fit the ATD and featured a medium contoured seatback (J3, Jay Seating Systems) and a 2.8 in contoured seat cushion (Ride Designs). Figure 2 illustrates the test set up.
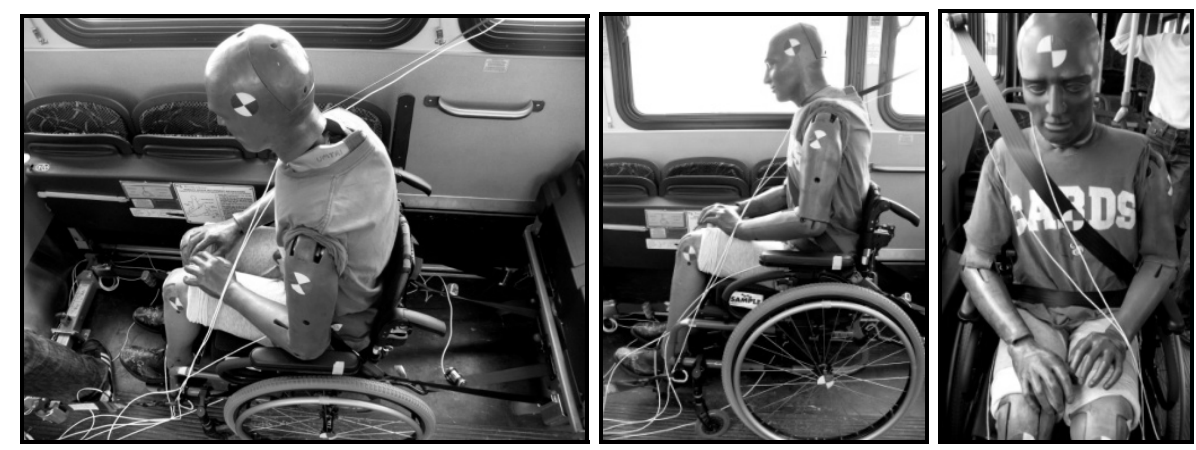

Figure 2A. Typical test setup of $50^{\text {th }}$ percentile ATD seated in manual wheelchair with four tiedowns and lap and shoulder belt applied. Low-g accelerometers were mounted in the ATD head, chest, and pelvis as well as on the LATV floor (at securement station). Load cells were used to measure webbing tension in the tiedowns and lap and shoulder belts. 


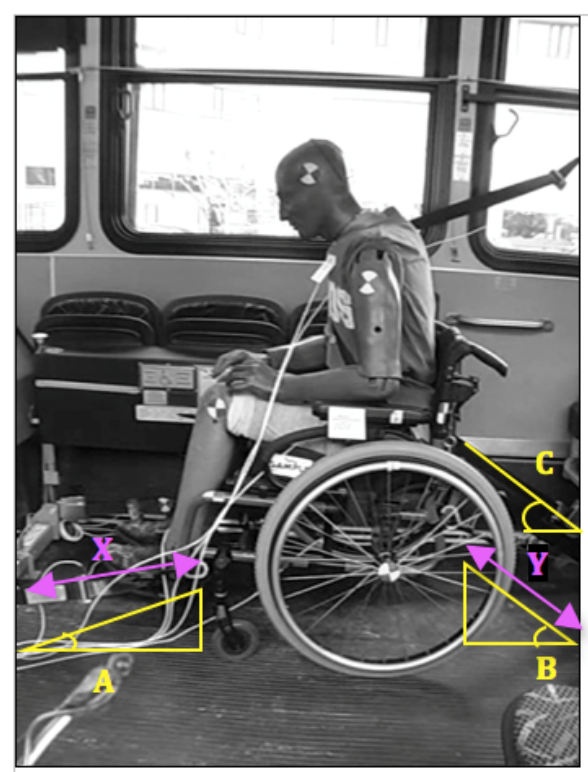

Side View

A - Front tiedown angle (side view) B - Rear tiedown angle (side view) $\mathrm{C}$ - Lap belt angle (side view)

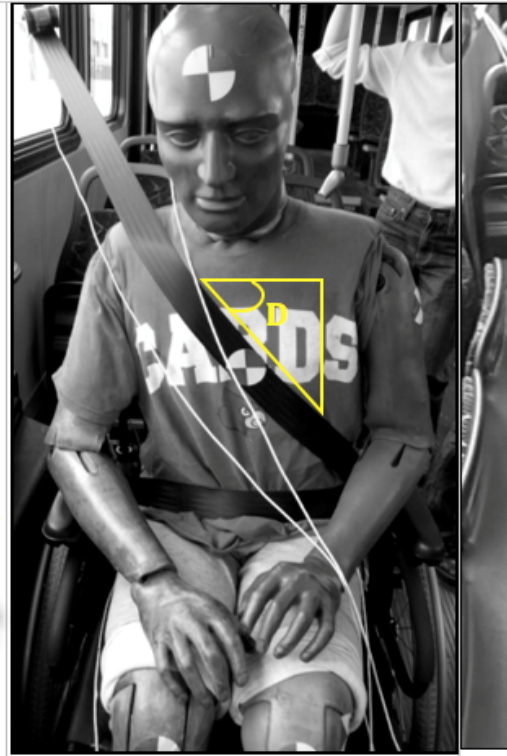

Front View (close)

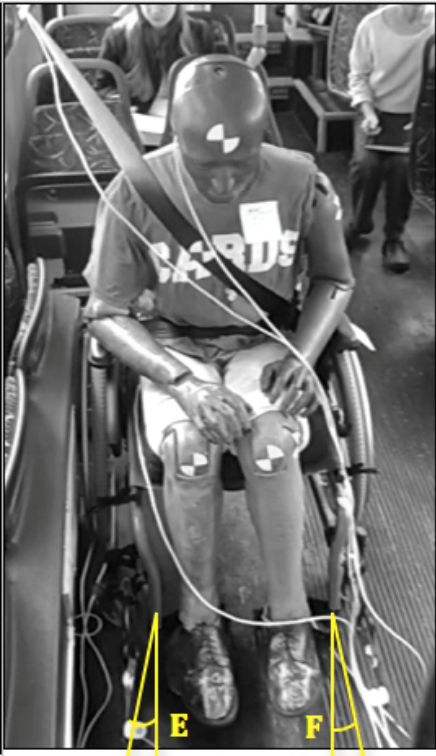

Front View (far)

D - Shoulder belt angle (front view)

$\mathrm{X}$ - Front tiedown length

$\mathrm{E}$ - Front right tiedown angle (front view) $\mathrm{Y}$ - rear tiedown length

$\mathrm{F}$ - Front left tiedown angle (front view)

Figure 2B. Test setup measurements obtained during test setup.

\begin{tabular}{|c|c|c|c|c|c|c|c|c|c|c|c|c|c|c|}
\hline Test & $\begin{array}{l}\text { FRTD } \\
\text { length }\end{array}$ & $\begin{array}{l}\text { FLTD } \\
\text { length }\end{array}$ & $\begin{array}{l}\text { FRTD } \\
\text { angle, } \\
\text { front- } \\
\text { view }\end{array}$ & $\begin{array}{l}\text { FLTD } \\
\text { angle, } \\
\text { front- } \\
\text { view }\end{array}$ & $\begin{array}{c}\text { FRTD } \\
\text { angle, } \\
\text { side- } \\
\text { view }\end{array}$ & $\begin{array}{c}\text { FLTD } \\
\text { angle, } \\
\text { side- } \\
\text { view }\end{array}$ & $\begin{array}{l}\text { RRTD } \\
\text { length }\end{array}$ & $\begin{array}{l}\text { RLTD } \\
\text { length }\end{array}$ & $\begin{array}{c}\text { RRTD } \\
\text { angle, } \\
\text { top- } \\
\text { view }\end{array}$ & $\begin{array}{c}\text { RLTD } \\
\text { angle, } \\
\text { top- } \\
\text { view }\end{array}$ & $\begin{array}{c}\text { RRTD } \\
\text { angle, } \\
\text { side- } \\
\text { view }\end{array}$ & $\begin{array}{c}\text { RLTD } \\
\text { angle, } \\
\text { side- } \\
\text { view }\end{array}$ & $\begin{array}{c}\text { LB } \\
\text { angle, } \\
\text { side- } \\
\text { view }\end{array}$ & $\begin{array}{c}\text { SB } \\
\text { angle, } \\
\text { front- } \\
\text { view }\end{array}$ \\
\hline ig 3 & $\mathrm{X}$ & $\mathrm{x}$ & E & $\mathrm{F}$ & A & A & $\mathrm{Y}$ & $\mathrm{Y}$ & -.. & -.. & B & B & C & D \\
\hline alue & $22 \frac{1}{2}$ in & $22 \frac{1}{4}$ in & $9.0^{\circ}$ & $6.8^{\circ}$ & $14.4^{\circ}$ & $13.5^{\circ}$ & $251 / 4$ in & $261 / 4$ in & $4.0^{\circ}$ & $30.0^{\circ}$ & $20.3^{\circ}$ & $18.8^{\circ}$ & $31.0^{\circ}$ & $27.0^{\circ}$ \\
\hline
\end{tabular}

Legend:

FRTD - Front Right Tiedown FLTD - Front Left Tiedown RRTD - Rear Right Tiedown $\quad$ RLTD - Rear Left Tiedown LB - Lap belt SB - Shoulder belt

Table 1. Experimental test set up WTORS measurements.

The wheelchair was secured at the securement station on the right side of the LATV (previous experience showed that wheelchair passengers use the right securement station). The securement station within the LATV conformed to ADA regulation with respect to size (measured size: 66 in long by 36 in wide), and featured a four-point, straptype, retractor-style WTORS (shoulder belt: SURE-LOK, FE200936M-AMS, lap belt: 
SURE-LOK, AL701060M-AMS, rear tiedowns: SURE-LOK, DAL 3300, front tiedowns:

AL700963S-AMS) that were used to secure the wheelchair and restrain the occupant.

The wheelchair-seated passenger represented by the ATD was instrumented with low-g, tri-axial accelerometers $(0 \pm 6 \mathrm{~g}$, Freescale Semiconductors, Inc., Model \# MMA7260Q) positioned at the head center of gravity (CG) and inside the thorax and pelvis instrumentation cavities, while the wheelchair was equipped with a tri-axial accelerometer at its CG. An additional tri-axial accelerometer $(0 \pm 6 \mathrm{~g}$, Freescale Semiconductors, Inc., Model \# MMA7260Q) was mounted on the floor of the LATV securement station (geometric center). The WTORS were instrumented with three-bar belt load cells (Denton Corp.), measuring tension forces in the webbing during testing. The load cells were calibrated up to $60 \mathrm{lb}$ at $5 \mathrm{lb}$ increments and zeroed prior to testing. WTORS retractors determined the pretension in all webbing. All acceleration and loading data was acquired at $500 \mathrm{~Hz}$ using a portable on-board customized data acquisition system. To minimized noise, this data was filtered using a Butterworth low pass filter. Fourier Transform revealed an appropriate cutoff frequency of $20 \mathrm{~Hz}$.

Statistical analysis was performed using SPSS v. 12.0.1 for Windows. Outcome measures (WTORS loading, ATD accelerations, and LATV accelerations) were evaluated for assumptions of normality using the Kolmogorov-Smirnov and ShapiroWilk statistical tests. If assumptions of normality were met, differences between outcomes measures within each maneuver were evaluated using repeated measures analysis of variance (ANOVA) and dependent means t-tests, while differences between outcome measures across various maneuvers were evaluated with one-way ANOVA and independent means t-test. When assumptions of normal data distribution were not met, 
non-parametric equivalent statistical tests (Friedman and the Wilcoxon Signed Ranks post hoc tests for within maneuver comparison; Kruskal-Wallis and the Mann-Whitney U post hoc tests for across maneuver comparison) were used to compare means. Statistical significance was set at $\mathrm{p} \leq 0.05$ and in cases when multiple comparisons were made Bonferroni correction was used to control the familywise error rate.

\section{Results}

Representative LATV acceleration time histories during acceleration, braking, left turn and right turn maneuvers are shown in Figure 3. Lateral LATV accelerations were negligible during acceleration and braking maneuvers.
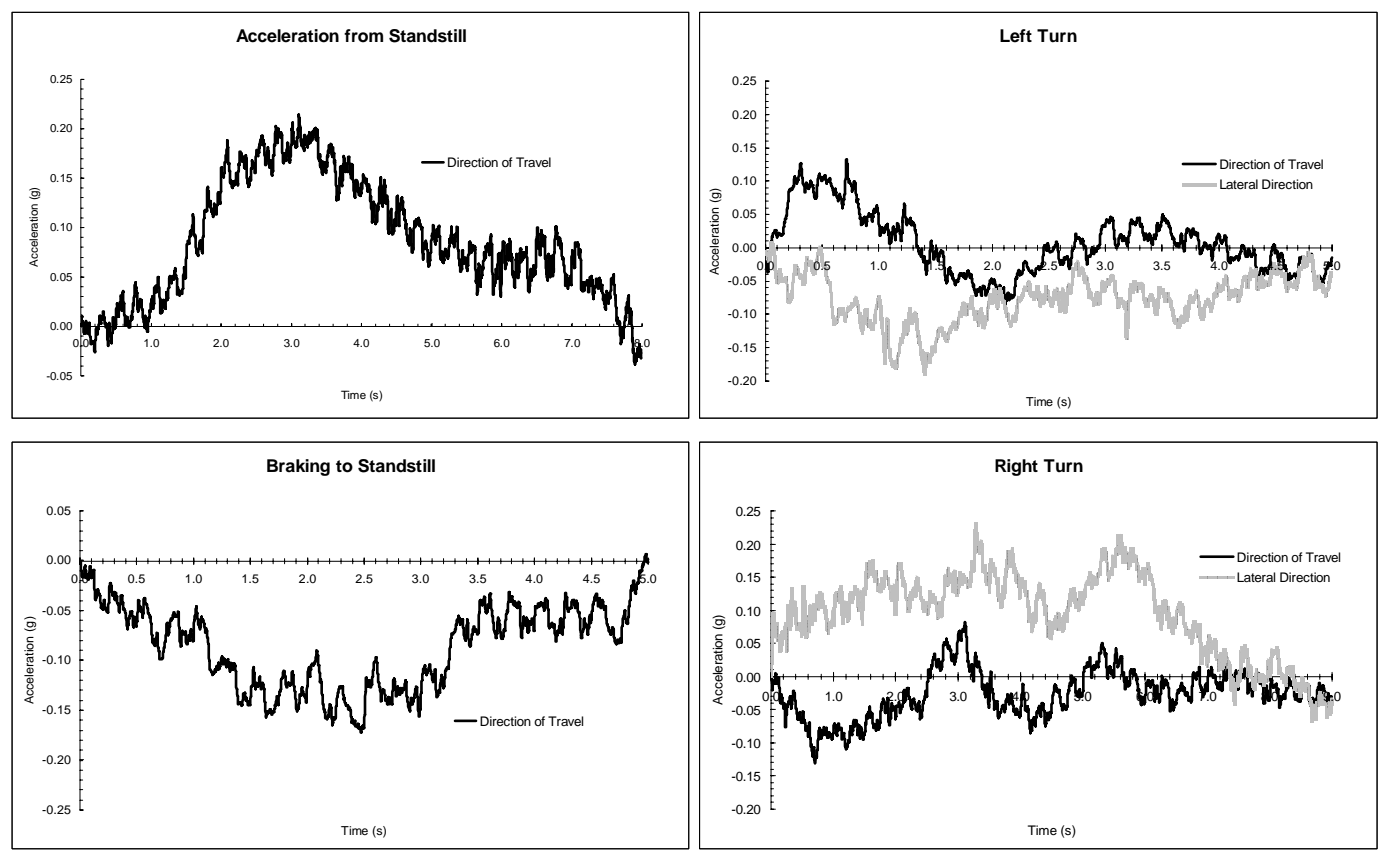

Figure 3. Typical LATV acceleration time histories for acceleration, braking, left turn, and right turn maneuvers under normal driving conditions. 
Figure 4 shows the mean peak LATV and ATD accelerations under normal driving conditions for accelerations from standstill, braking to standstill, left turns, and right turns. An attempt was made to measure wheelchair acceleration, but a defective accelerometer rendered wheelchair acceleration data unattainable.

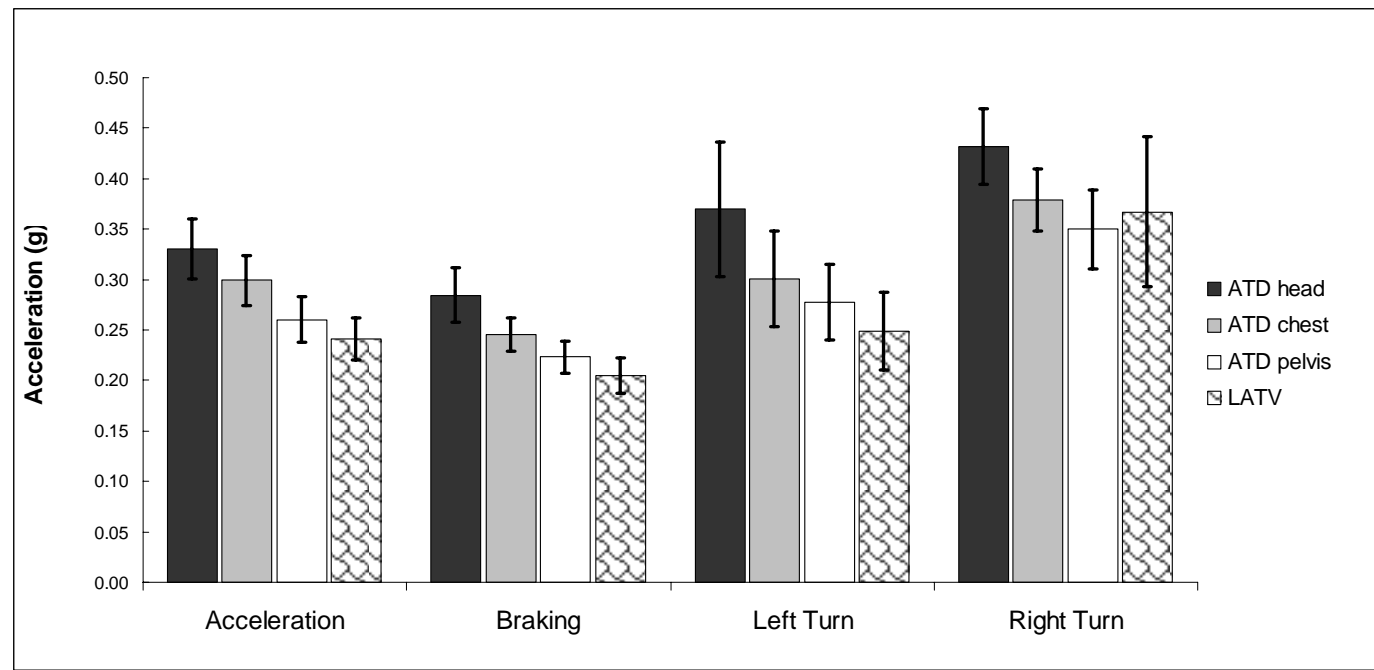

Figure 4. Mean peak resultant accelerations for ATD and LATV for acceleration $(n=87)$, braking $(n=81)$, left turn $(n=10)$, and right turn maneuvers $(n=10)$ under normal driving conditions. (Error bars indicate $95 \%$ confidence intervals).

The highest mean peak ATD accelerations occurred during right turns $(0.43 \mathrm{~g}$ head; $0.38 \mathrm{~g}$ chest; $0.35 \mathrm{~g}$ pelvis). Accelerations failed to meet assumptions of normality. Non-parametric analysis revealed no statistical differences across maneuvers for the ATD head, chest, and pelvis. Across all maneuvers, the overall peak resultant ATD accelerations (ATD head $1.07 \mathrm{~g}$, ATD chest $0.89 \mathrm{~g}$, and ATD pelvis $0.78 \mathrm{~g}$ ) occurred during acceleration maneuvers. The overall peak LATV acceleration of $0.70 \mathrm{~g}$ was recorded during a right turn maneuver.

Figures 5-8 illustrate mean peak WTORS loading during acceleration, braking, left, and right turns under normal LATV driving. [Sample size for the right rear tiedown 
(RRTD) was limited compared to other WTORS because the RRTD load cell failed during testing].

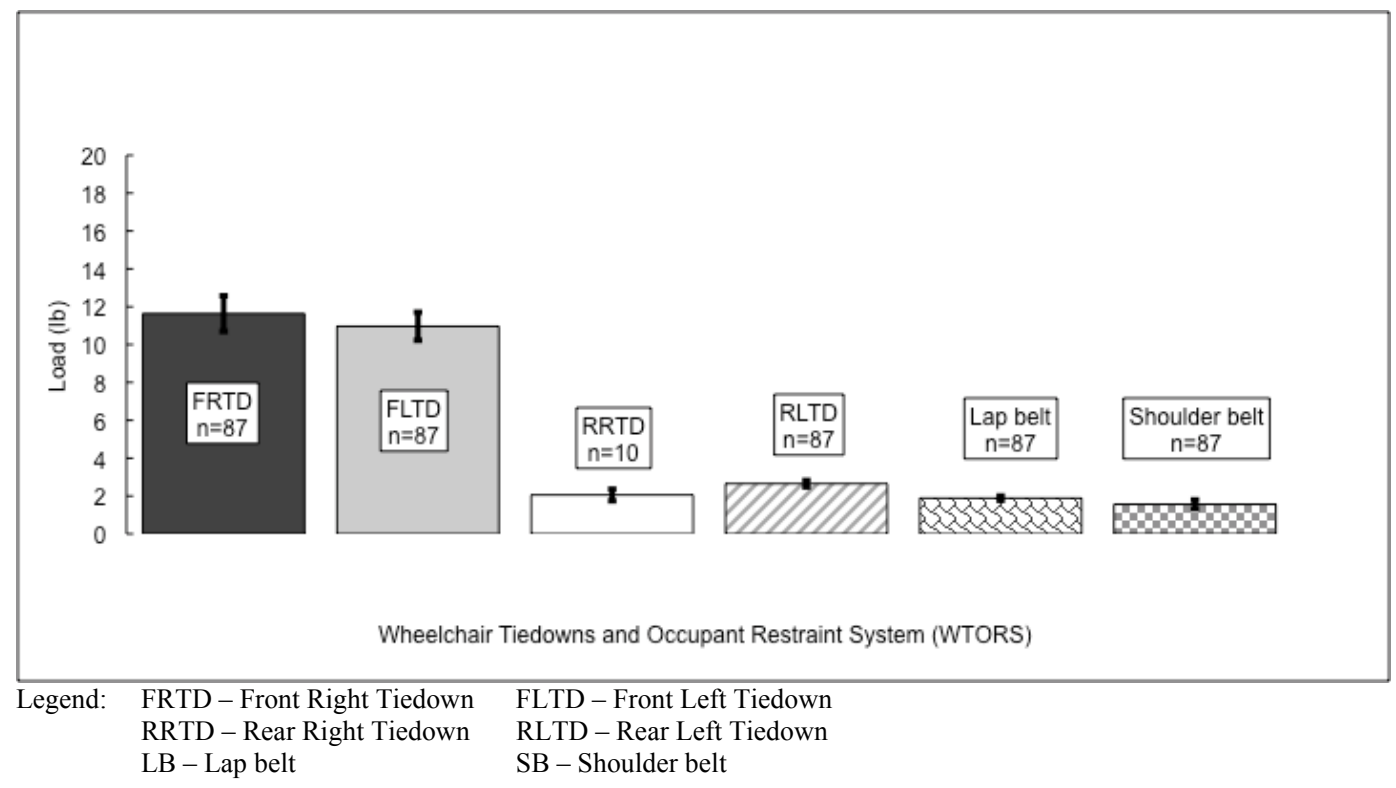

Figure 5. Mean peak WTORS loading for acceleration (from standstill) maneuvers under normal LATV driving conditions. (Error bars indicate $95 \%$ confidence intervals).

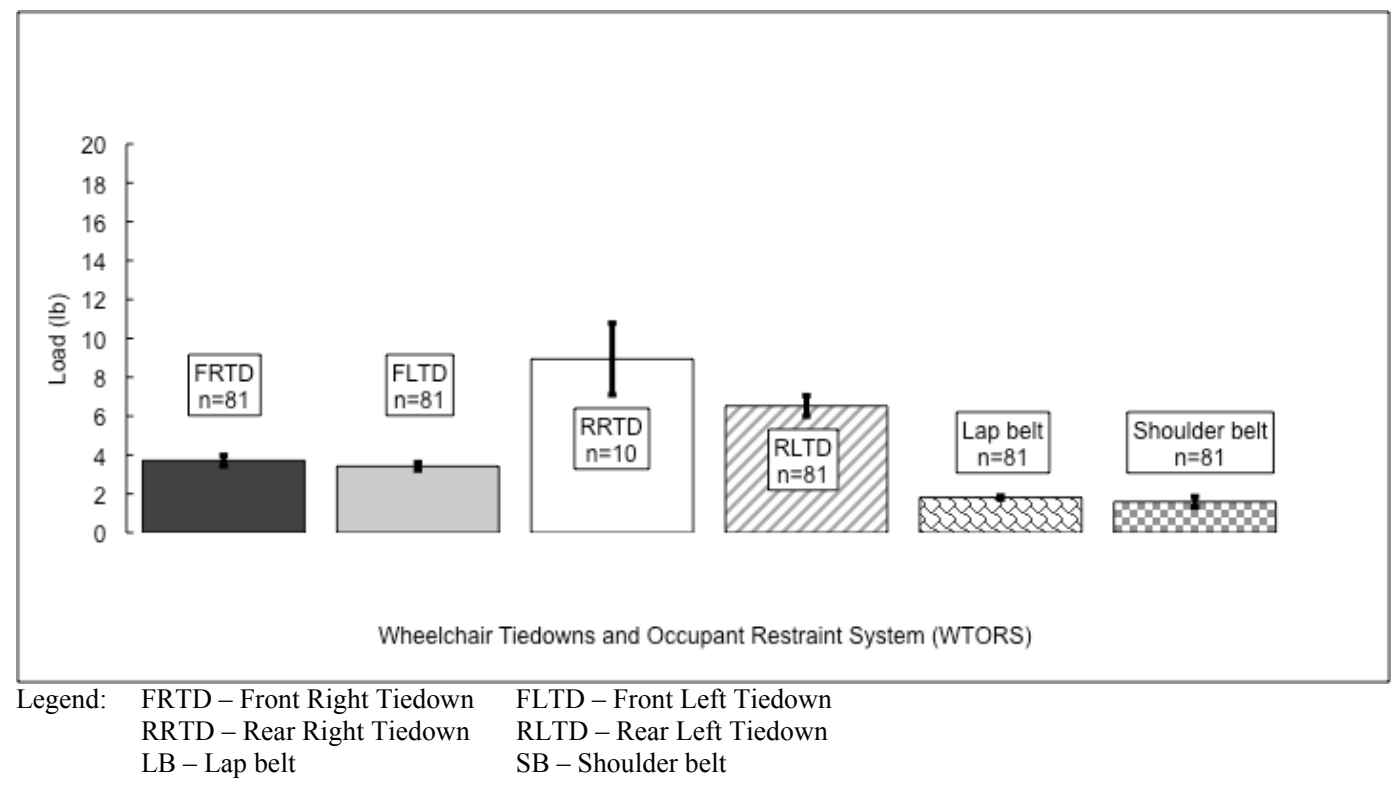

Figure 6. Mean peak WTORS loading for braking (to standstill) maneuvers under normal LATV driving conditions. (Error bars indicate 95\% confidence intervals). 


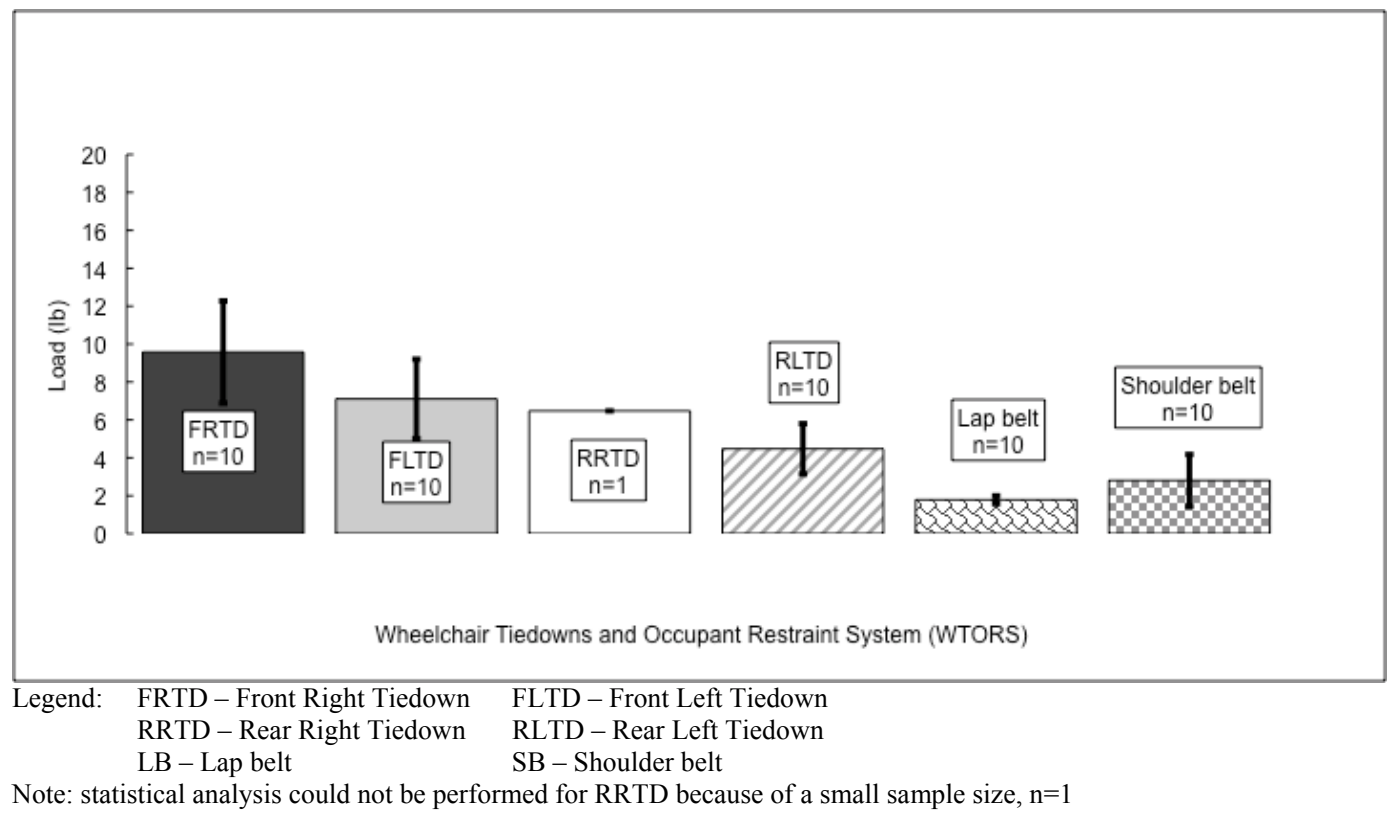

Figure 7. Mean peak WTORS loading for left turn maneuvers under normal LATV driving conditions. (Error bars indicate 95\% confidence intervals).

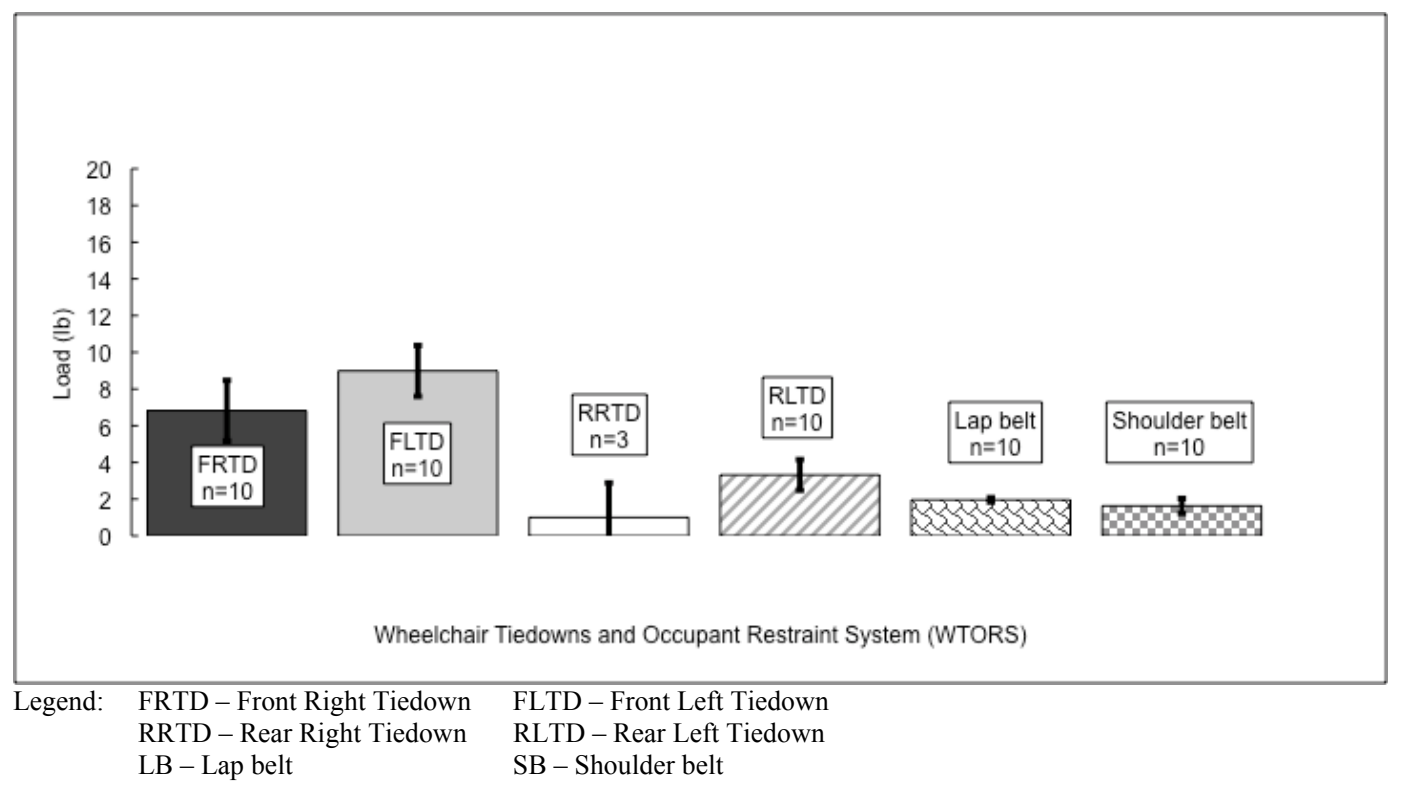

Figure 8. Mean peak WTORS loading for right turn maneuvers under normal LATV driving conditions. (Error bars indicate 95\% confidence intervals).

LATV acceleration maneuvers produced the highest mean peak loads $(11.0 \mathrm{lb}$

FRTD). Loading data failed to meet assumptions of normality, thus non-parametric tests 
were used for comparison. During acceleration maneuvers the mean peak loading for the front tiedowns was significantly higher than the mean peak loading for the rear tiedowns (FRTD vs. RRTD, $\mathrm{p}=0.007$ and FLTD vs. RLTD, $\mathrm{p}<0.001$ ). During braking maneuvers

the mean peak loading for the rear tiedowns was significantly higher than that of the front tiedowns (RRTD vs. FRTD, $\mathrm{p}=0.005$ and RLTD vs. FLTD, $\mathrm{p}<0.001$ ). LATV braking maneuvers led to the highest mean peak loads for the rear tiedowns ( $9.0 \mathrm{lb}$ for RRTD, 6.5 $\mathrm{lb}$ for RLTD). The overall peak front tiedown loading (FRTD $19.0 \mathrm{lb}$, FLTD $16.4 \mathrm{lb}$ ) was recorded during acceleration maneuvers, while the overall peak rear tiedown loading (RRTD 14.8 lb, RLTD $14.3 \mathrm{lb}$ ) was recoded during braking maneuvers.

For left turn maneuvers mean peak FLTD loading was significantly lower than mean peak FRTD loading $(\mathrm{p}=0.005)$. During right turns mean peak FRTD loading was significantly lower than mean peak FLTD loading $(\mathrm{p}=0.009)$. Additionally, during right turns mean peak FLTD loading was significantly higher than mean peak RLTD loading $(\mathrm{p}=0.007)$. No other significant differences were identified during turning.

Mean peak LB loading was negligible across all maneuvers $(\leq 2 \mathrm{lb})$. The mean peak SB loading was also negligible across all maneuvers $(\leq 3 \mathrm{lb})$.

\section{Discussion and Conclusions}

The LATV accelerations found in our study indicate that the mean peak resultant accelerations for acceleration maneuvers $(0.24 \mathrm{~g})$ are slightly higher than those for braking maneuvers $(0.21 \mathrm{~g})$. Mean peak resultant LATV accelerations during left turns $(0.25 \mathrm{~g})$ were higher than during braking maneuvers $(0.21 \mathrm{~g})$, while mean peak resultant LATV accelerations during right turns $(0.37 \mathrm{~g})$ were highest overall [greater than during 
left turn $(0.25 \mathrm{~g})$, acceleration $(0.24 \mathrm{~g})$, and braking maneuvers $(0.21 \mathrm{~g})]$. Higher magnitude in LATV resultant acceleration for right turn maneuvers can be attributed to higher lateral accelerations. Mean peak LATV accelerations were greater for right turns vs. left turns, given that the turning radius was typically much smaller for right turns than left turns (during right turns the LATV did not have to "cross the intersection" as it did during left turns).

Zaworski, et al. ${ }^{11}$ reported peak LATV accelerations for various normal driving maneuvers on multiple routes and for a variety of LATV designs and models. In their study, they reported that LATV forward accelerations (accelerations from standstill) ranged from $0 \mathrm{~g}$ to $0.20 \mathrm{~g}$, left turn and right turn accelerations ranged from $0.05 \mathrm{~g}$ to $0.40 \mathrm{~g}$, while LATV accelerations for stopping maneuvers ranged from $0.05 \mathrm{~g}$ to $0.40 \mathrm{~g}$. The findings in our study fall within these reported ranges, with the exception of our mean peak accelerations from standstill being slightly higher $(0.24 \mathrm{~g})$. Another study by van Roosmalen, et al. ${ }^{29}$ reported maximum LATV accelerations of $0.60 \mathrm{~g}$ during normal driving maneuvers; a range of $0.56 \mathrm{~g}$ to $0.75 \mathrm{~g}$ (mean $0.64 \mathrm{~g}$, standard deviation, SD $0.1 \mathrm{~g}$ ) for braking maneuvers, and $0.38 \mathrm{~g}$ to $0.56 \mathrm{~g}$ (mean $0.47 \mathrm{~g}$, SD $0.1 \mathrm{~g}$ ) for turning maneuvers. The mean peak accelerations in our study were lower than those reported by van Roosmalen, et al. ${ }^{29}$ across all maneuvers. Variations in our findings compared to other studies may be attributed to the different LATV manufacturers and models, LATV suspension systems, routes, terrain, and LATV operator driving. Kamper, et al. ${ }^{26}$ used vehicle accelerations of $0.2 \mathrm{~g}$ and $0.4 \mathrm{~g}$ to assess wheelchair passenger postural response to vehicle perturbations in normal driving. Sprigle and Linden ${ }^{24}$ also studied accelerations wheelchair passengers experience in modified van and reported vehicle accelerations of 
0.084-0.250g during acceleration, $0.063-0.719 \mathrm{~g}$ during braking, and $0.133-0.701 \mathrm{~g}$ during turning. In another study ${ }^{23}$, they reported mean total vehicle accelerations of $0.5 \mathrm{~g}$ and less in van under various normal driving maneuvers and across various positions in the vehicle (driver, front, and rear). The mean peak accelerations in our study fall within the vehicle acceleration ranges reported by Kamper, et al. ${ }^{26}$ and Sprigle and Linden ${ }^{23,24}$.

For each ATD accelerometer location (head, chest and pelvis), the mean peak accelerations were greatest during right and left turn maneuvers, followed by accelerations from standstill, and braking to standstill. LATV accelerations paralleled this, so it may be concluded that this acceleration pattern was transferred to the ATD. Sprigle and Linden ${ }^{24}$ also reported mean wheelchair passenger trunk accelerations under normal driving conditions in a modified van: acceleration $(0.028-0.151 \mathrm{~g})$, braking $(0.086-$ $0.363 \mathrm{~g})$, and turning maneuvers $(0.062-0.194 \mathrm{~g})$. The ATD mean chest accelerations in our study of LATV normal maneuvers were higher than those found by Sprigle and Linden ${ }^{24}$ in van normal driving, with the exception of those occurring during braking maneuvers. Since vehicle accelerations in our study and the Sprigle and Linden study ${ }^{24}$ were comparable, differences in wheelchair passenger chest accelerations may be explained by our use of an ATD to represent a wheelchair passenger and Sprigle and Linden using human wheelchair passengers with spinal cord injuries. Additional factors such as the wheelchair, and wheelchair seat cushion that can contribute to differences in wheelchair passenger accelerations were reported by Linden and Sprigle in a separate study ${ }^{23}$.

The front tiedowns exhibited higher mean peak loads than the rear tiedowns during LATV acceleration maneuvers, while the rear tiedowns showed higher mean peak 
loading during LATV braking maneuvers. This loading pattern is consistent with the dynamics the wheelchair is subjected to during these maneuvers: LATV braking causes forward wheelchair excursions, loading the rear tiedowns, while LATV acceleration causes aft wheelchair excursions, loading the front tiedowns. The lap and shoulder belt mean peak loading in both LATV acceleration and braking maneuvers was negligible. Even though shoulder belt loading appeared slightly higher in left turns vs. right turns (2.8 lb vs. $1.6 \mathrm{lb}$ ), this difference was not statistically significant.

During all maneuvers, we observed minor ATD chest and head excursions, while observing negligible excursions for the ATD pelvis and wheelchair. Minor ATD chest and head excursions are substantiated by relatively low ATD chest and pelvis accelerations across all maneuvers (highest mean peak ATD acceleration, measured at the head was $0.43 \mathrm{~g}$ ). Wheelchair and ATD instability can have a direct impact on ATD accelerations and WTORS loading. The relatively low ATD accelerations and low WTORS loading in this study indicate that proper WTORS use is associated with low wheelchair and wheelchair passenger instability in LATV normal driving.

Tiedown geometry can also affect tiedown loading. The tiedown asymmetry in this study was a function, in part, of anchor point location in the securement station of the LATV used in this study. Even though asymmetric tiedown geometry existed, the tiedowns were in compliance with recognized standards (SAE J2249 ${ }^{14}$, ISO 10542-2 ${ }^{19}$, and $\mathrm{ADA}{ }^{13}$ ). It should be pointed out that the securement point locations on the wheelchair can also affect the overall geometry of the tiedown system. Differently sized wheelchairs, with different securement point locations would have produced different tiedown geometry, which may have influenced WTORS loading. 
During left turn maneuvers, mean peak FLTD loading was significantly lower than mean peak FRTD loading. During right turn maneuvers, mean peak FRTD loading was significantly lower than mean peak FLTD loading. This loading pattern was not expected since the dynamics the wheelchair is typically subjected to during turning maneuvers is as follows: LATV right turns causes tendency for wheelchair excursions to the left, loading the right tiedowns, while LATV left turns causes tendency for wheelchair excursions to the right, loading the left tiedowns. There are several factors that could have influenced FRTD and FLTD loading during LATV turning maneuvers. Firstly, the folding seat under the window (depicted in Figures 2 and 3) served to limit wheelchair tendency for movement to the right side, thus reducing FLTD loading during left turns. Additionally, the LATV lateral tilt (LATV right side lower than left side) due to the road tilt for rain-water run off in the right lane may have produced increased FLTD loading during right turns. Finally, the asymmetric tiedown geometry (Table 1) could have influenced front tiedown loading during LATV turning maneuvers. Even though the mean difference between FRTD and FLTD peak loading (2.3lb for right turns, 2.5lb for left turns) was statistically significant, this difference is likely not meaningful when considering tiedown design guidelines (design tolerances are expected to be higher). The LATV was usually at or near standstill before performing any turning maneuver; LATV forward acceleration resulted in increased front tiedown loading compared to rear tiedown loading (FLTD was significantly higher than RLTD loading during right turns). It is important to note that statistical analysis of rear tiedown loading during turning maneuvers was difficult: comparison between mean peak RRTD and RLTD could not be made for left turns because of limited sample size $(n=1)$, while mean peak RRTD and 
RLTD loading for right turns were not significantly different (perhaps due to small sample size, $n=3$ ). In these instances, limited sample size is because of associated load cell failure during testing.

As anticipated, the mean peak WTORS loading in our study was lower than WTORS loading reported in other studies ${ }^{30-34}$ evaluating high-g deceleration environments. In our study, across all maneuvers the mean peak load range per tiedown was 3.4-11.6 $\mathrm{lb}$ for front tiedowns, $1.0-8.9 \mathrm{lb}$ for rear tiedowns, $1.8-2.0 \mathrm{lb}$ for the $\mathrm{LB}$, and 1.6-2.8 $\mathrm{lb}$ for the SB. The maximum peak loading per tiedown was $19.0 \mathrm{lb}$ for front tiedowns, $14.8 \mathrm{lb}$ for rear tiedowns, $4.9 \mathrm{lb}$ for $\mathrm{LB}$, and $7.3 \mathrm{lb}$ for SB. In previous studies simulating high-g frontal impacts ${ }^{30,32,33}$, the peak front tiedown load (per tiedown) ranged from 22.5-471.0 lb, while the peak rear tiedown load (per tiedown) ranged from 1400.1-4728.4 lb. Rear impact studies ${ }^{31,34}$ reported peak front tiedown loads per tiedown ranging from 1079.1-1765.0 lb, while peak rear tiedown loads per tiedown ranged from 57.8-427.1 lb. As expected, WTORS loads measured in our study for normal driving conditions are substantially less than those during simulated impact events. One other study ${ }^{35}$ reported mean estimated LATV accelerations of up to $2.60 \mathrm{~g}$ during rear impact crashes, which is substantially higher than the highest mean peak LATV acceleration of $0.37 \mathrm{~g}$ found in our study. Since most WTORS are designed for high-g (crash) environments, their robust design requirement may reduce ease of use and need for alternative WTORS designs.

Our study and the aforementioned studies ${ }^{30-34}$ utilized a strap-type, 4-point WTORS system; this system conforms to both national and international standards (SAE $\mathrm{J} 2249^{14}$, ISO $10542-2^{19}$ ) as well as ADA requirements ${ }^{8}$ and is designed to withstand 
loading associated with a high-g crash environment. ADA requirements ${ }^{8}$ include static loading thresholds of at least $2500 \mathrm{lb}$ per tiedown (and at least $5000 \mathrm{lb}$ for entire securement system), while SAE J2249 ${ }^{14}$ and ISO $10542-2{ }^{19}$ have performance requirements that incorporate dynamic testing (frontal impact, 20g, 30mph). However, as previously mentioned, problems with WTORS ease and frequency of use have been reported $^{3,21}$. In a previous study, we reported that complete use of tiedowns (application of all four tiedowns) to secure wheelchairs was observed in only $7.5 \%$ of wheelchair passenger trips in LATVs, while tiedown misuse (application of one to three tiedowns) was observed in $18.9 \%$ of trips and misuse of the lap belt in an attempt to secure the wheelchair was common (44\% of trips $)^{21}$. Additionally, we found in our previous study that the majority $(73.6 \%)$ of observed wheelchair passenger trips in LATVs had evidence of nonuse (zero tiedowns used) during transit ${ }^{21}$. Current research is underway to identify alternative solutions to wheelchair securement in a low-g environment $(<1 \mathrm{~g})$. These alternative solutions include forward-facing ${ }^{22}$ and rear-facing ${ }^{17}$ wheelchair passenger stations and auto-docking systems ${ }^{18}$. They offer improved ease of use and may exhibit reduced frequencies of disuse and misuse in comparison to the strap-type, 4-point WTORS systems. Rear facing wheelchair passenger stations require the wheelchair passenger to back their wheelchair up to a forward excursion barrier that would prevent forward excursions in case of a sudden stop and may feature a lateral excursion barrier to prevent wheelchair rotation into the aisle, as well. Auto-docking systems require additional hardware that interlocks an adaptor mounted on the wheelchair and the docking system installed in the vehicle. Both of these alternatives to traditional straptype, 4-point WTORS offer a more independent approach for wheelchair securement or 
retention, but future research is needed to demonstrate their feasibility and provision of safety. Our study provided data on LATV accelerations and WTORS loading during normal driving conditions, which may prove helpful in advancing these alternative approaches to wheelchair securement or retention and passenger restraint.

There are several limitations associated with our study. An ATD was used to represent the wheelchair passenger, which may not be an ideal representation. The ATD was designed based on the $50^{\text {th }}$ percentile anthropometric measures of the general male population; these measures may not be the same for the wheelchair passenger population. Additionally, the ATD was developed and validated for use in a high-g (20g) crash environment, while our study was conducted in a low-g $(<1 \mathrm{~g})$ setting. The ATD's joint properties may be too stiff to accurately simulate actual wheelchair passenger response in a low-g environment. However, as surrogate humans based on the wheelchair population and/or surrogate humans validated for low-g environments have not yet been developed, the ATD used in this study is a reasonable first step in representing a wheelchair passenger.

The wheelchair used in our study was an ultra-light, $\mathrm{x}$-braced frame, manual wheelchair; other wheelchairs may have produced different tiedown loading. Also, our study was limited to only one LATV make and model, one LATV operator, and one LATV route; varying LATV models, LATV operators, and LATV routes with differing topography may have produced different LATV accelerations resulting in different ATD accelerations and tiedown loading.

Our study also failed to provide wheelchair accelerations during normal driving maneuvers, because of a malfunctioning accelerometer. Wheelchair acceleration data 
would have provided an additional measure describing the kinematic environment experienced by a wheelchair passenger while the LATV is in transit. Additionally, the sample size for the right rear tiedown (RRTD) was limited because the associated load cell failed during testing. Restrictions on time and use of the LATV did not allow us to repeat testing to gather these data.

Finally, our findings only described normal LATV driving maneuvers. Emergency driving maneuvers will likely be associated with increased LATV and ATD accelerations, as well as WTORS loading. (Concurrent efforts are underway by the authors to analyze data from similar LATV emergency driving maneuvers.) While this study describes the kinetic environment of normal LATV driving maneuvers experienced by a wheelchair and wheelchair passenger, it does not provide insight into consequences of WTORS disuse and misuse under normal LATV driving conditions.

In conclusion, our study found the highest mean peak LATV accelerations during right turn maneuvers. The mean peak LATV accelerations in our study were comparable or slightly lower than peak LATV accelerations in other published studies. ATD accelerations (head, chest and pelvis) were greatest during rights and left turns, followed by accelerations, and braking maneuvers. Mean peak ATD accelerations increased moving from inferior to superior ATD locations due to an "articulation effect" of the ATD. Mean peak front tiedown loading in our study was highest during LATV acceleration maneuvers, while mean peak rear tiedown loading was highest during LATV braking maneuvers. Right-side mean peak tiedown loading was higher in comparison to left-side mean peak tiedown loading for right turn maneuvers, while left-side mean peak tiedown loading was higher than right-side mean peak tiedown loading for left turn 
maneuvers. WTORS loading measured in our study was substantially less than WTORS loading reported in other studies with high-g (crash) deceleration environments. Our study provided LATV and ATD accelerations and WTORS loading during LATV normal driving conditions; data that may prove helpful in advancing alternative approaches to wheelchair securement/retention and passenger restraint, such as the forward-facing and rear-facing wheelchair passenger stations and auto-docking systems. 


\title{
CHAPTER 4
}

\author{
KINETIC ENVIRONMENT OF A MANUAL WHEELCHAIR SEATED PASSENGER \\ ONBOARD A LARGE ACCESSIBLE TRANSIT VEHICLE DURING EMERGENCY \\ DRIVING MANEUVERS
}

\begin{abstract}
Overview
The goal of this portion of the study was to describe the kinetic environment experienced by a wheelchair-seated passenger onboard an LATV under emergency driving conditions. This study also described consequences of WTORS disuse and misuse during LATV emergency driving maneuvers. WTORS disuse was defined as complete non-use of all WTORS belts (no occupant restraints, zero tiedowns), while WTORS misuse was defined as the lap belt routed between the passenger and the wheelchair seatback in an attempt to secure the wheelchair. An anthropomorphic testing device (ATD) represented the wheelchair passenger, while the wheelchair used in this study was a transit, manual, X-braced-frame wheelchair. The ATD was restrained using occupant restraints (lap and shoulder belt) while the wheelchair was secured using 4-point, strap type tiedowns. For this study the LATV performed the following emergency driving maneuvers on a closed course: braking, acceleration, left turn, right turn, left turn while braking, right turn while braking, and abrupt lane changes. The LATV acceleration,
\end{abstract}


wheelchair acceleration, ATD head acceleration, ATD chest acceleration, lap and shoulder belt loads along with tiedown loads were recorded for all maneuvers, while front- and side-view video data was also collected. We found that LATV and ATD head and chest accelerations, in addition to WTORS loads are higher during emergency driving than normal driving. When WTORS are properly applied, the wheelchair passenger does not appear to be at increased risk of injury. WTORS disuse and misuse are associated with a high frequency of wheelchair passenger ejection from the wheelchair.

\section{Introduction}

Since large accessible transit vehicles (LATVs) have low fatality incidence of all passengers ${ }^{1}$, they are generally considered a safe mode of transportation. Major reasons include overall vehicle mass and low velocity of LATV travel. Despite this relatively safe environment, Shaw and Gillispie ${ }^{3}$ found that wheelchair passengers have an accident rate over 350 times greater than that of ambulatory passengers. The U.S. National Highway Transportation Safety Administration (NHTSA) reported that 35\% of injuries and deaths that occurred between 1990-1995 involving wheelchair-seated passengers or drivers were likely due to improper or lack of securement of the wheelchair, and $17 \%$ of these incidents occurred in LATVs ${ }^{4}$.

The vast majority of surveyed wheelchair passengers $(85 \%)$ remain seated in their

wheelchair during transit, which may put them at increased risk of injury ${ }^{10}$. Songer, et al. found that passengers who remained seated in their wheelchair during transit experienced higher injury rates (5.2 passenger injuries per 100,000 miles) than those who transferred to a motor vehicle seat ( 0.6 passenger injuries per 100,000 miles $)^{6}$. 
It is necessary to afford wheelchair passengers the same level of transportation safety as the general population. The Americans with Disabilities Act (ADA) ${ }^{13}$ requires that LATVs be equipped with a wheelchair securement device and lap and shoulder belts. A common method to secure the wheelchair and restrain the wheelchair occupant is with the use of four-point, strap type wheelchair tiedown and occupant restraint systems (WTORS). Although the ADA requires the provision of a wheelchair securement device and occupant restraints (lap and shoulder belts), it does not mandate the use of such systems ${ }^{8}$. Individual transit agencies are left to establish policies requiring the use of wheelchair tiedowns. Additionally, the ADA does not require mandatory use of lap and/or shoulder belt restraints for wheelchair-seated passengers unless the same mandate has been made for the general ridership ${ }^{8}$. As a result, there are disparities between transit agency policies with respect to WTORS use ${ }^{10}$.

Disuse and misuse of WTORS have been shown to place wheelchair-seated passengers at greater risk of injury. Shaw and Gillispie ${ }^{3}$ identified misuse as a factor contributing to wheelchair passengers injuries. In our previous study ${ }^{7}$, we reviewed video surveillance records of WTORS usage on LATVs and found for cases when passengers remained seated in wheelchairs during transit, the majority ( $76 \%$ ) of wheelchairs were not secured using four point strap-type tiedowns during transport, and that misuse of the lap belt was common ( $44 \%$ of cases). Misuse of the lap belt often consisted of the LATV operators attempting to secure the wheelchair by routing the lap belt around the wheelchair seatback in an attempt to secure the wheelchair. Reported disuse and misuse of four-point, strap-type tiedowns and occupant restraint systems (ORS) in the LATV low-g environment may be evidence that these WTORS are not user 
friendly and do not allow for wheelchair passenger independence with respect to wheelchair securement and passenger restraint. Alternative solutions to wheelchair securement or retention such as auto-docking ${ }^{18}$ and rear-facing stations ${ }^{17}$ are being investigated in an attempt to increase ease of use and independence for wheelchair passenger securement and restraint.

One study by Zaworski, et al. ${ }^{11}$ has described in part the low-g LATV environment (LATV accelerations for various normal and emergency driving maneuvers). Another study by Wolf, et al. ${ }^{15}$ used an unvalidated computer simulation model to investigate effects of WTORS configuration on wheelchair passenger safety in during normal LATV maneuvers. Previous studies by Sprigle and Linden ${ }^{23,24}$ focused on describing the dynamic environment wheelchair passengers encounter while traveling in a van (reporting vehicle and wheelchair passenger accelerations). Kamper, et al. ${ }^{25-27}$ developed a technique and portable system to assess postural response of wheelchair passengers in vehicles. However, we are not aware of any studies to-date that describe the kinetic environment (accelerations and loads) a manual wheelchair passenger experiences during LATV emergency driving. By describing WTORS loading, ATD accelerations and LATV accelerations, our study provides data that may advance alternative WTORS methods in the LATV environment.

\section{Methods}

This experimental study focused on describing the kinetic environment experienced by a surrogate wheelchair passenger during emergency LATV driving maneuvers. The LATV (Gillig, Year: 2010, Model \# G30D102N4) used in the 
experimental evaluation was a hybrid, $40 \mathrm{ft}(12.2 \mathrm{~m})$, low floor, pneumatic suspension, fixed-route, intra-city bus. The emergency driving course was based on a previous study ${ }^{11}$ and designed to maximize LATV acceleration. We attempted to drive the course three times (closed off to the public, Figure 1). The course included maximum acceleration from standstill ("pedal-to-the-metal" up to $30 \mathrm{mph}$ ), right turn, left turn, lane change, braking ("panic stop") while turning left (from $30 \mathrm{mph}$, braking/left-turn), braking ("panic stop") while turning right (from $30 \mathrm{mph}$, braking/right-turn), and braking ("panic stop" from $30 \mathrm{mph}$ ) maneuvers.

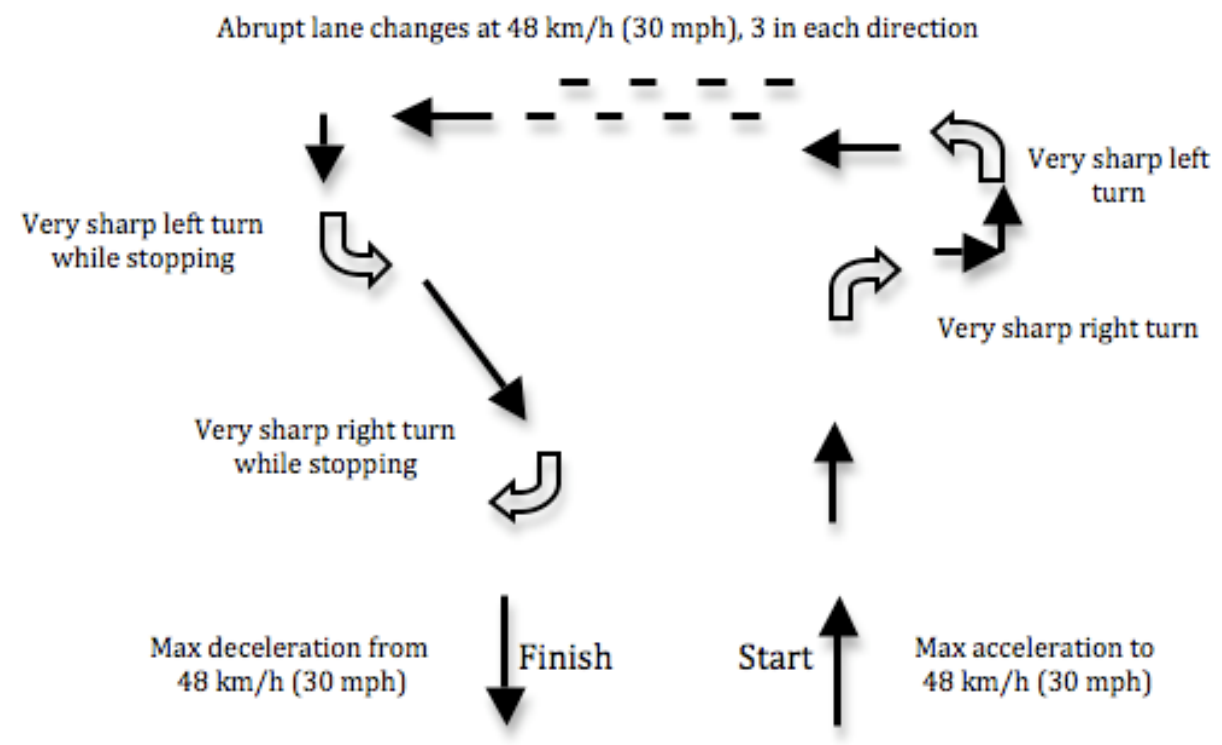

Figure 1. Emergency driving course conducted on a closed driving course.

Testing was performed in dry weather conditions and excluding the research group, no passengers were on board the LATV during testing. The same licensed LATV operator was used during all testing and was instructed to perform all emergency maneuvers uniformly and in a manner that would maximize LATV accelerations without compromising vehicle stability and safety. 
The wheelchair passenger was represented by a wheelchair-seated, $50^{\text {th }}$ percentile Hybrid II anthropomorphic testing device (ATD - $172 \mathrm{lb}, 78.2 \mathrm{~kg}$ ), while the wheelchair (Quickie 2, Sunrise Medical) used during testing was an ultra-light, X-braced, manual wheelchair with the transit option (conforming to the ISO 7176-19 standard "Wheeled mobility devices for use as seats in motor vehicles" ${ }^{28}$ ). The wheelchair was adjusted to properly fit the ATD and featured a medium contoured seatback (J3, Jay Seating Systems) and a 2.8 in contoured seat cushion (Ride Designs).

The wheelchair was secured at the securement station on the right side of the LATV (previous experience showed that wheelchair passengers use the right side more often). The securement station on the LATV conformed to ADA regulation with respect to size (measured size: 66 in by 36 in), and featured four-point, strap-type, retractor-style WTORS (shoulder belt: SURE-LOK, FE200936M-AMS, lap belt: SURE-LOK, AL701060M-AMS, rear tiedowns: SURE-LOK, DAL 3300, front tiedowns: AL700963SAMS).

Table 1 below shows a test matrix, detailing the three WTORS configurations evaluated in this study. The WTORS consisted of the front right tiedown (FRTD), front left tiedown (FLTD), rear right tiedown (RRTD), rear left tiedown (RLTD), and the lap belt (LB) and shoulder belt (SB). Because of limited resources (LATV time and LATV operator time), the WTORS configurations in this study were limited and do not include all possible WTORS combinations. We attempted to drive the emergency driving course (Figure 1) three times for each WTORS configuration (resulting in sample size $\mathrm{n}=3$ for each maneuver under each WTORS configuration). The wheelchair and ATD were reset for each maneuver trial. 


\begin{tabular}{|c|c|c|c|}
\hline & \multicolumn{3}{|c|}{ Occupant Restraint System (ORS) } \\
\hline $\begin{array}{c}\text { Tiedowns } \\
\text { Applied }\end{array}$ & None & $\begin{array}{c}\text { Lap belt misused to } \\
\text { secure wheelchair * }\end{array}$ & $\begin{array}{c}\text { Correct use of ORS } \\
\text { (lap \& shoulder belt) }\end{array}$ \\
\hline 0 & A & B & \\
\hline 4 & & & C \\
\hline
\end{tabular}

Legend: A, B, C-Combinations of tiedowns and occupant restraints (ORS) evaluated during driving experiments * - Commonly implemented WTORS configuration ${ }^{7}$

Table 1. Test Matrix of WTORS configurations used in emergency LATV driving.

Combinations of WTORS to be evaluated were determined based on previous findings: monitoring of wheelchair activities has given insight into the patterns of fourpoint, strap-type tiedowns and occupant restraint use. Findings have shown that the use of WTORS on LATVs varies from the worst-case scenario with no use of occupant restraint system (ORS) and zero tiedowns (A) to the ideal and best-case circumstance with correct use of ORS (both lap \& shoulder belt) and all four tiedowns (C). Previous findings also indicated that the lap belt is often misused to secure the wheelchair during transit: the lap belt is routed around the seatback only, and makes no contact with the wheelchair passenger (B). The test matrix shows three WTORS configurations (A, B, and C) evaluated and is intended to represent a range of conditions, including the misuse of the lap belt to secure the wheelchair.

Figure 2 illustrates the general test set up, and Table 2 shows WTORS measurements when the WTORS were properly employed (WTORS configuration C). Figure 3 shows how the lap belt was misused in an attempt to secure the wheelchair (WTORS configuration B). 

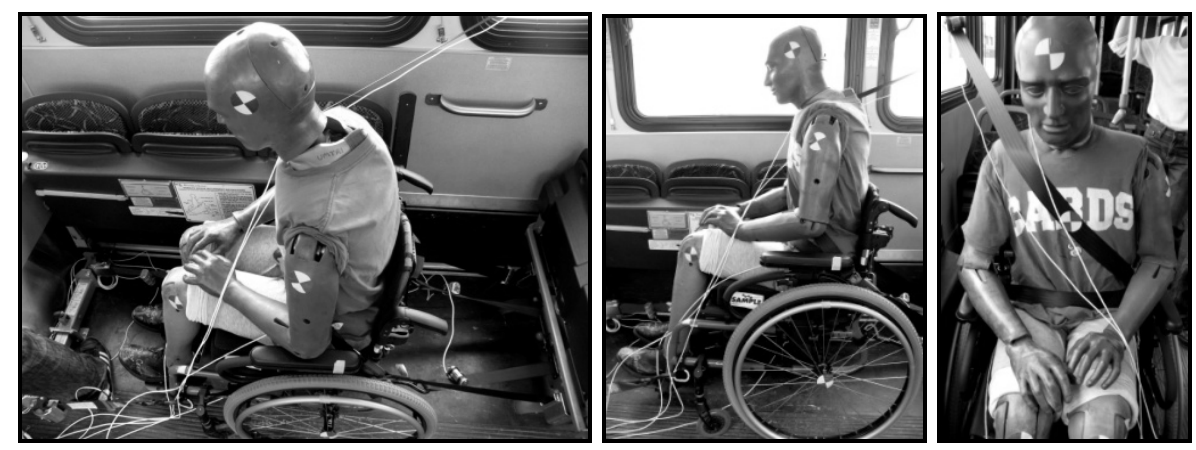

Figure 2A. Typical test setup of $50^{\text {th }}$ percentile ATD seated in manual wheelchair with four tiedowns and lap and shoulder belt applied. Low-g accelerometers were mounted in the ATD head, chest, and pelvis as well as the LATV floor (at securement station) while load cells were used to measure webbing tension in the tiedowns and lap and shoulder belts.

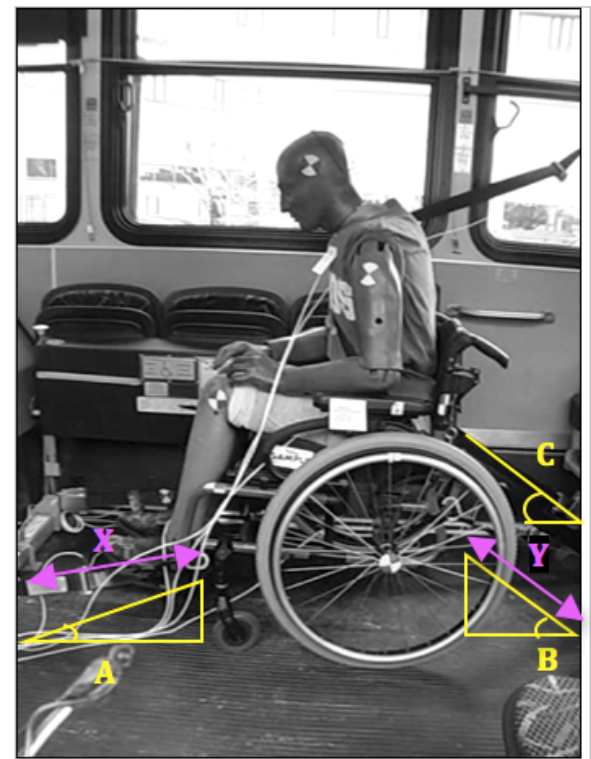

Side View

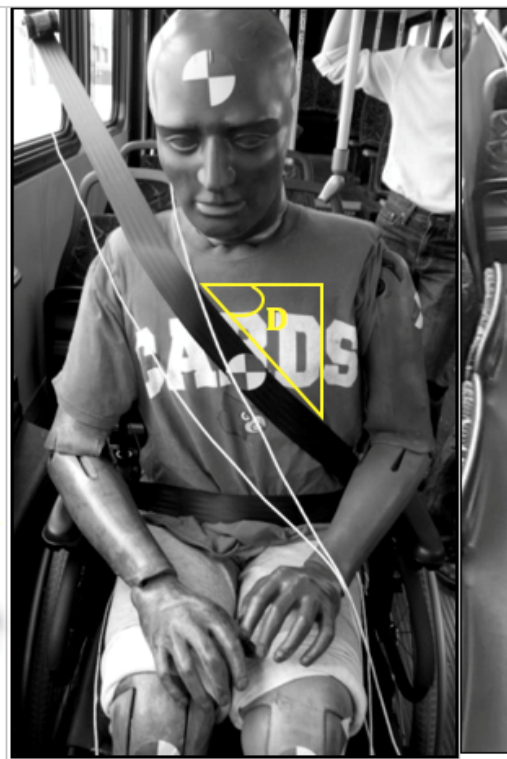

Front View (close)

A - Front tiedown angle (side view)

$\mathrm{B}$ - Rear tiedown angle (side view)

C - Lap belt angle (side view)

$\mathrm{D}$ - Shoulder belt angle (front view)

$\mathrm{E}-$ Front right tiedown angle (front view)

$\mathrm{F}-$ Front left tiedown angle (front view)

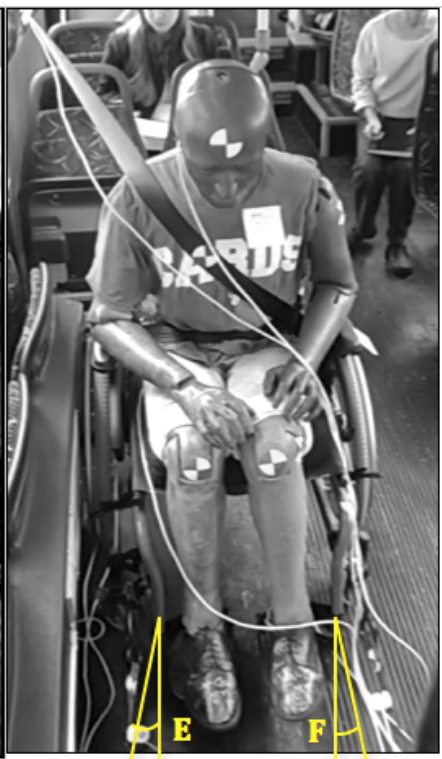

Front View (far)

$\mathrm{X}$ - Front tiedown length

$\mathrm{Y}$ - rear tiedown length

Figure 2B. Test setup measurements obtained during test setup. 


\begin{tabular}{|c|c|c|c|c|c|c|c|c|c|c|c|c|c|c|}
\hline Test & $\begin{array}{l}\text { FRTD } \\
\text { length }\end{array}$ & $\begin{array}{l}\text { FLTD } \\
\text { length }\end{array}$ & $\begin{array}{l}\text { FRTD } \\
\text { angle, } \\
\text { front- } \\
\text { view }\end{array}$ & $\begin{array}{l}\text { FLTD } \\
\text { angle, } \\
\text { front- } \\
\text { view }\end{array}$ & $\begin{array}{c}\text { FRTD } \\
\text { angle, } \\
\text { side- } \\
\text { view }\end{array}$ & $\begin{array}{c}\text { FLTD } \\
\text { angle, } \\
\text { side- } \\
\text { view }\end{array}$ & $\begin{array}{l}\text { RRTD } \\
\text { length }\end{array}$ & $\begin{array}{l}\text { RLTD } \\
\text { length }\end{array}$ & $\begin{array}{c}\text { RRTD } \\
\text { angle, } \\
\text { top- } \\
\text { view }\end{array}$ & $\begin{array}{l}\text { RLTD } \\
\text { angle, } \\
\text { top- } \\
\text { view }\end{array}$ & $\begin{array}{c}\text { RRTD } \\
\text { angle, } \\
\text { side- } \\
\text { view }\end{array}$ & $\begin{array}{l}\text { RLTD } \\
\text { angle, } \\
\text { side- } \\
\text { view }\end{array}$ & $\begin{array}{c}\text { LB } \\
\text { angle, } \\
\text { side- } \\
\text { view }\end{array}$ & $\begin{array}{c}\text { SB } \\
\text { angle, } \\
\text { front- } \\
\text { view }\end{array}$ \\
\hline Fig 3 & $\mathrm{X}$ & $\mathrm{X}$ & E & $\mathrm{F}$ & A & A & $\mathrm{Y}$ & Y & -- & $--\cdot$ & B & B & $\mathrm{C}$ & D \\
\hline Value & $22 \frac{1}{2}$ in & $221 / 4$ in & $9.0^{\circ}$ & $6.8^{\circ}$ & $14.4^{\circ}$ & $13.5^{\circ}$ & $251 / 4$ in & $261 / 4$ in & $4.0^{\circ}$ & $30.0^{\circ}$ & $20.3^{\circ}$ & $18.8^{\circ}$ & $31.0^{\circ}$ & $27.0^{\circ}$ \\
\hline
\end{tabular}

Legend: $\begin{array}{ll}\text { FRTD }- \text { Front Right Tiedown } & \text { FLTD }- \text { Front Left Tiedown } \\ \text { RRTD - Rear Right Tiedown } & \text { RLTD - Rear Left Tiedown } \\ \text { LB }- \text { Lap belt } & \text { SB }- \text { Shoulder belt }\end{array}$

Table 2. Experimental test set up WTORS measurements.

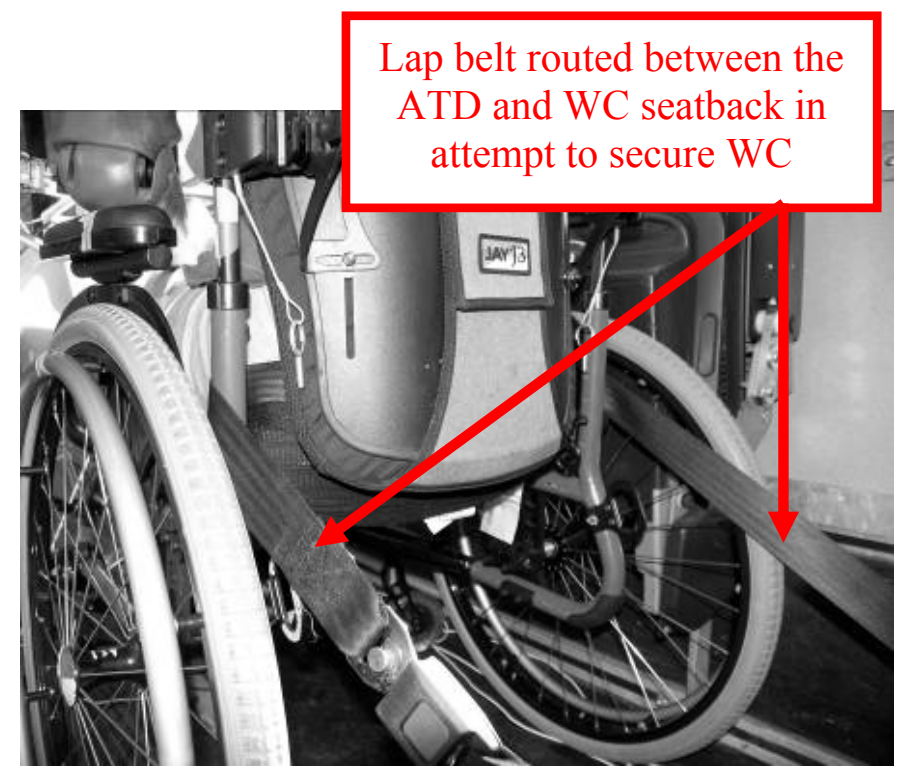

Figure 3. Test setup of $50^{\text {th }}$ percentile ATD seated in manual wheelchair with lap belt used in an attempt to secure the wheelchair (routed around the seatback, WTORS configuration B). The tiedowns and shoulder belt were not applied.

The wheelchair-seated passenger represented by the ATD was instrumented with low-g, tri-axial accelerometers $(0 \pm 6 \mathrm{~g}$, Freescale Semiconductors, Inc., Model \# MMA7260Q) positioned at the head center of gravity (CG), and inside the thorax and pelvis instrumentation cavities. The wheelchair was equipped with a tri-axial accelerometer at its CG. An additional tri-axial accelerometer $(0 \pm 6 \mathrm{~g}$, Freescale Semiconductors, Inc., Model \# MMA7260Q) was placed at the securement station on the 
floor of the LATV. The WTORS were instrumented with three-bar belt load cells (Denton Corp.), measuring tension forces in the webbing during testing. The load cells were zeroed after placement within the belt webbing. WTORS retractors determined the pretension in all webbing. All acceleration and loading data were acquired at $500 \mathrm{~Hz}$ using a portable on-board data acquisition system. To minimize noise, this data was filtered using a Butterworth low pass filter.

Statistical analysis was performed using SPSS v. 12.0.1 for Windows. Accelerations and WTORS loading data were evaluated for assumptions of normality using the Kolmogorov-Smirnov and Shapiro-Wilk statistical tests. If assumptions of normality were met, differences between outcomes measures within each maneuver were evaluated using repeated measures analysis of variance (ANOVA) and dependent means t-tests, while differences between outcome measures across various maneuvers were evaluated with one-way ANOVA and independent means t-test. When assumptions of normal data distribution were not met, non-parametric equivalent statistical tests (Friedman and the Wilcoxon Signed Ranks post hoc tests for within maneuver comparison; Kruskal-Wallis and the Mann-Whitney U post hoc tests for across maneuver comparison) were used to compare means. Statistical significance was set at $\mathrm{p} \leq 0.05$.

\section{Results}

Representative LATV acceleration time histories during emergency acceleration, braking, left turn, right turn, braking/left-turn, braking/right-turn, and lane change maneuvers are shown in Figure 4. Lateral LATV accelerations were negligible during acceleration and braking maneuvers. 
Despite our intention to drive the emergency driving course three times for each WTORS configuration, the number of trials for WTORS configurations A and B had to be limited to protect sensitive sensor and data acquisition equipment. The high frequency of ATD ejections endangered the equipment. A faulty accelerometer rendered ATD pelvic acceleration data unattainable for all emergency driving maneuvers.

Figure 5 shows the mean peak LATV resultant accelerations across all emergency driving maneuvers (independent of WTORS configuration). Even though deviance from normal distribution could not be confirmed because of limited sample sizes, previous experimental testing results in normal driving (chapter 3 ) lead to the assumption of nonnormality for both acceleration and loading data. Non-parametric analysis revealed that mean peak LATV resultant acceleration was significantly higher during braking $(0.87 \mathrm{~g})$ than during acceleration $(0.28 \mathrm{~g})$ maneuvers $(\mathrm{p}=0.003)$. The mean peak LATV resultant acceleration in right turns $(0.84 \mathrm{~g})$ was higher than in left turns $(0.72 \mathrm{~g})$, but this difference was not statistically significant $(\mathrm{p}=0.180)$. The mean peak LATV resultant acceleration in braking/left-turn maneuvers $(1.04 \mathrm{~g})$ was higher than in braking/right-turn maneuvers $(0.97 \mathrm{~g})$, but this difference was also not statistically significant $(\mathrm{p}=0.557)$. The highest overall peak LATV resultant acceleration (1.26g) across all maneuvers was measured during a braking/left-turn maneuver. 


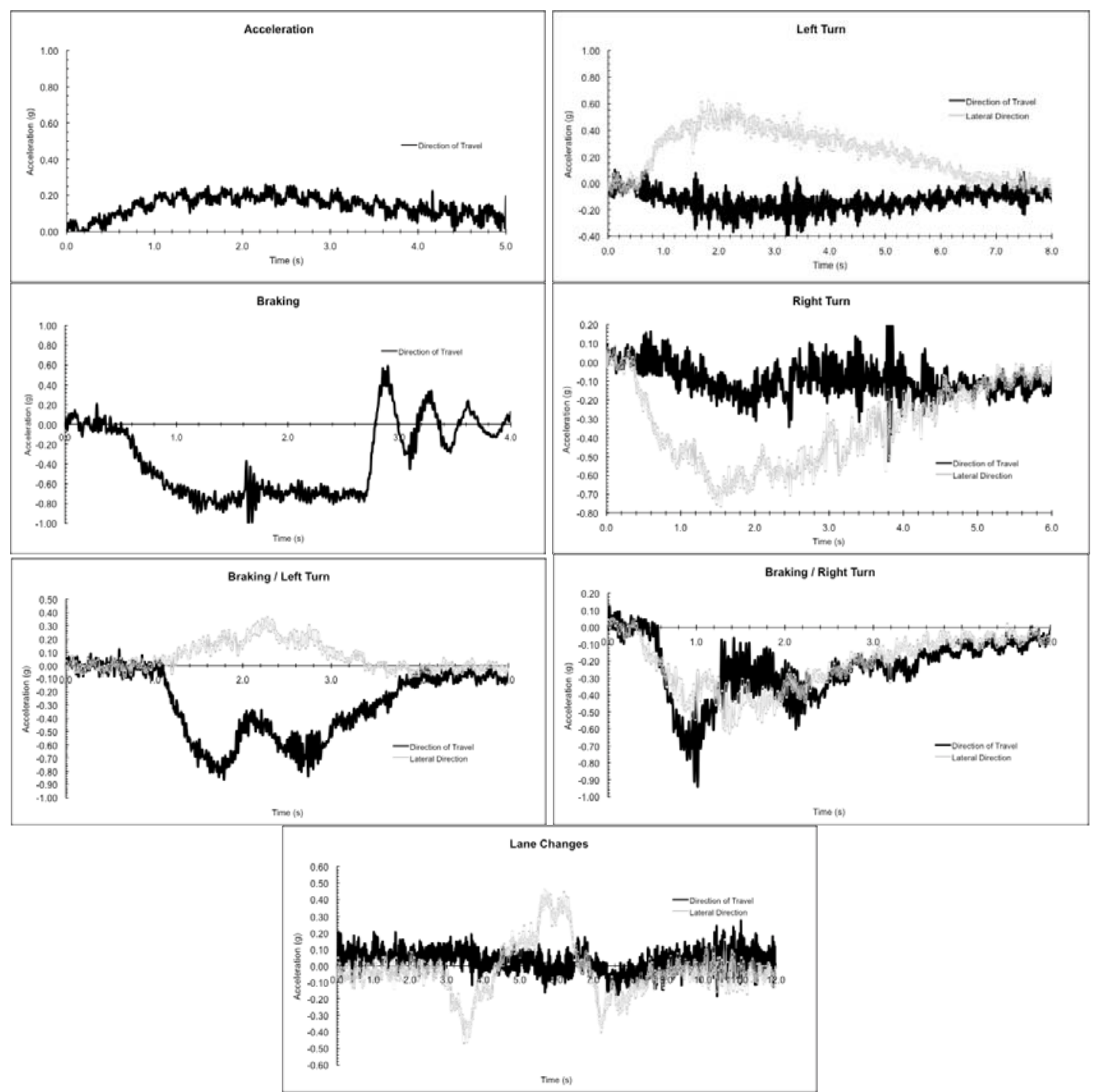

Figure 4. Representative LATV acceleration time histories for acceleration, braking, left turn, right turn, braking/left-turn, braking/right-turn, and lane change maneuvers under emergency driving conditions. 


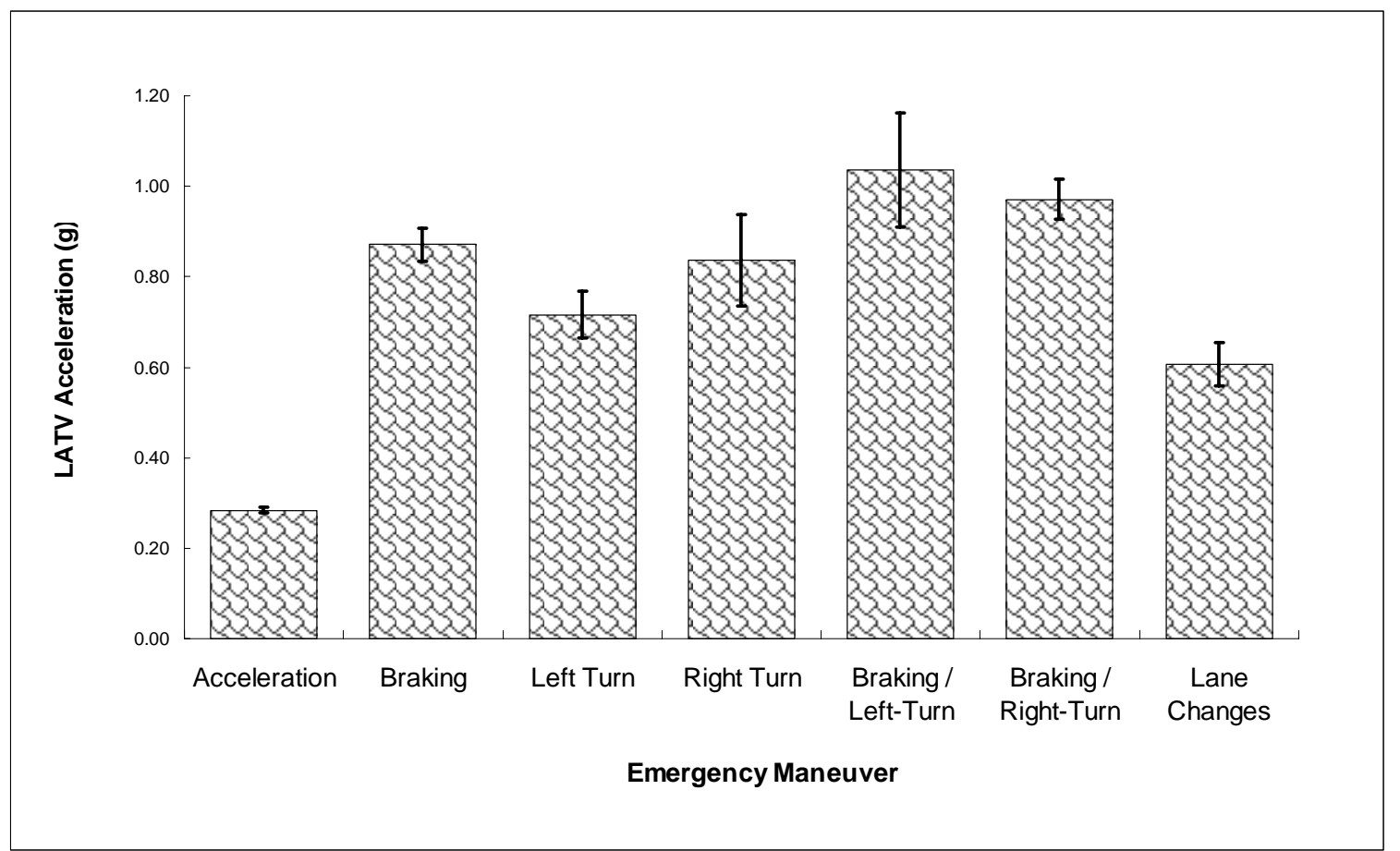

Figure 5. Mean peak LATV resultant accelerations (independent of WTORS configuration) for acceleration $(n=7)$, braking $(n=5)$, left turn $(n=6)$, right turn $(n=6)$, braking/left-turn $(n=4)$, braking/right-turn $(n=5)$, and lane change $(n=4)$ maneuvers under emergency driving conditions. (Error bars indicate 95\% confidence intervals).

Figure 6 shows mean peak ATD head, ATD chest, and wheelchair resultant accelerations across the LATV emergency maneuvers (with proper WTORS use, configuration C). An attempt was made to measure ATD pelvis acceleration, but a defective accelerometer rendered ATD pelvis acceleration data unattainable. Differences for mean peak ATD and wheelchair accelerations were not statistically significant (ATD head: acceleration vs. braking $\mathrm{p}=0.100$, left turn vs. right turn $\mathrm{p}=0.100$; ATD chest: acceleration vs. braking $\mathrm{p}=0.100$, left turn vs. right turn $\mathrm{p}=0.100$; and wheelchair: acceleration vs. braking $\mathrm{p}=0.100$, left turn vs. right turn $\mathrm{p}=0.100$ ). These differences were not statistically significant due to small sample size (small number of maneuvers). Sample size power analysis ${ }^{\dagger}$ revealed a minimum sample size of $n=21$ per group is

\footnotetext{
${ }^{\dagger}$ Online statistics calculator v. 3.0 (http://www.danielsoper.com)
} 
necessary to find statistical significance (for $1-\beta=0.80, \alpha=0.05$, and effect size Cohen's $\mathrm{d}=0.80$ ). For proper WTORS use (configuration $\mathrm{C}$ ), the highest overall peak wheelchair resultant acceleration (1.33g) occurred during a braking/left-turn maneuver, while the highest overall peak ATD chest $(3.15 \mathrm{~g})$ and head $(4.65 \mathrm{~g})$ resultant accelerations occurred during a braking/right-turn maneuver.

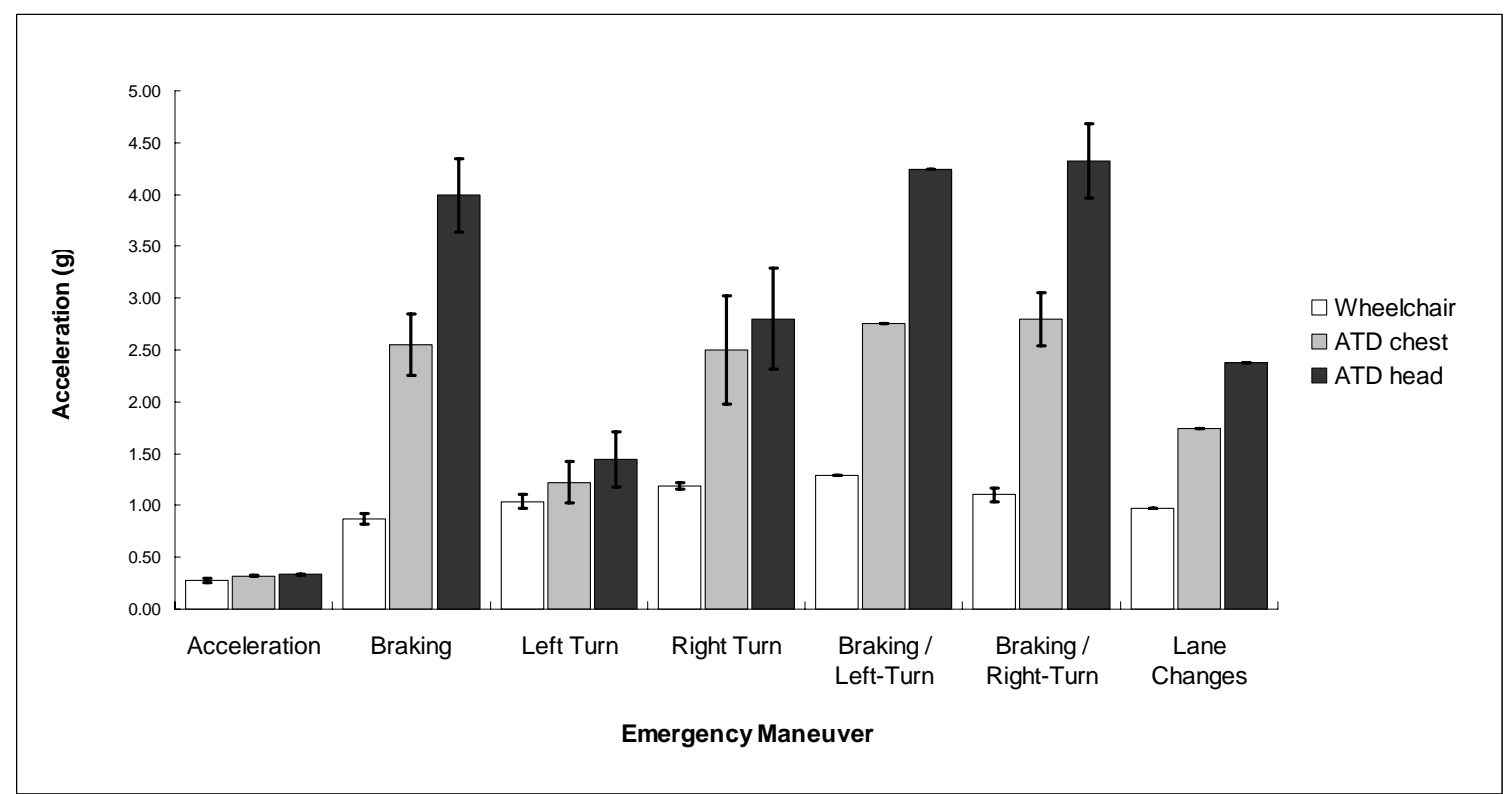

Figure 6. Mean peak resultant accelerations for ATD and wheelchair (proper WTORS use, configuration C) for acceleration $(\mathrm{n}=3)$, braking $(\mathrm{n}=3)$, left turn $(\mathrm{n}=3)$, right turn $(\mathrm{n}=3)$, braking/left-turn $(\mathrm{n}=2)$, braking/right-turn $(\mathrm{n}=3)$, and lane change $(\mathrm{n}=2)$ maneuvers under emergency driving conditions. (Error bars indicate $95 \%$ confidence intervals; error bars not shown for sample sizes $\mathrm{n}<3$ ).

Figures 7-13 show mean peak WTORS loading across each LATV emergency maneuver (with proper WTORS use, configuration C). The mean peak front tiedown loading is higher than mean peak rear tiedown loading (FRTD vs. RRTD, FLTD vs. RLTD) during acceleration maneuvers, while mean peak rear tiedown loads are higher than mean peak front tiedown loads during braking maneuvers. The right side tiedown loads are higher than the left side tiedown loads during right turns (FRTD vs. FLTD, RRTD vs. RLTD). For braking, braking/left-turn, and braking/right-turn maneuvers the 
shoulder belt loading is higher than the lap belt loading. However, because of small sample sizes $(\mathrm{n} \leq 3)$, we were not able to confirm that these differences in WTORS loading were statistically significant (power analysis revealed minimum sample size of $\mathrm{n}=21)$. The highest overall peak FRTD loading $(135.5 \mathrm{lb})$, the highest overall peak RRTD loading (138.4 lb), and the highest overall peak LB loading $(34.0 \mathrm{lb})$ were recorded during right turns. The highest overall peak FLTD loading $(53.0 \mathrm{lb})$ occurred during lane changes, while the highest peak RLTD loading $(44.9 \mathrm{lb})$ occurred during rightturn/braking. The highest overall peak SB loading (107.2 lb) occurred during rightturn/braking and left-turn/braking maneuvers.

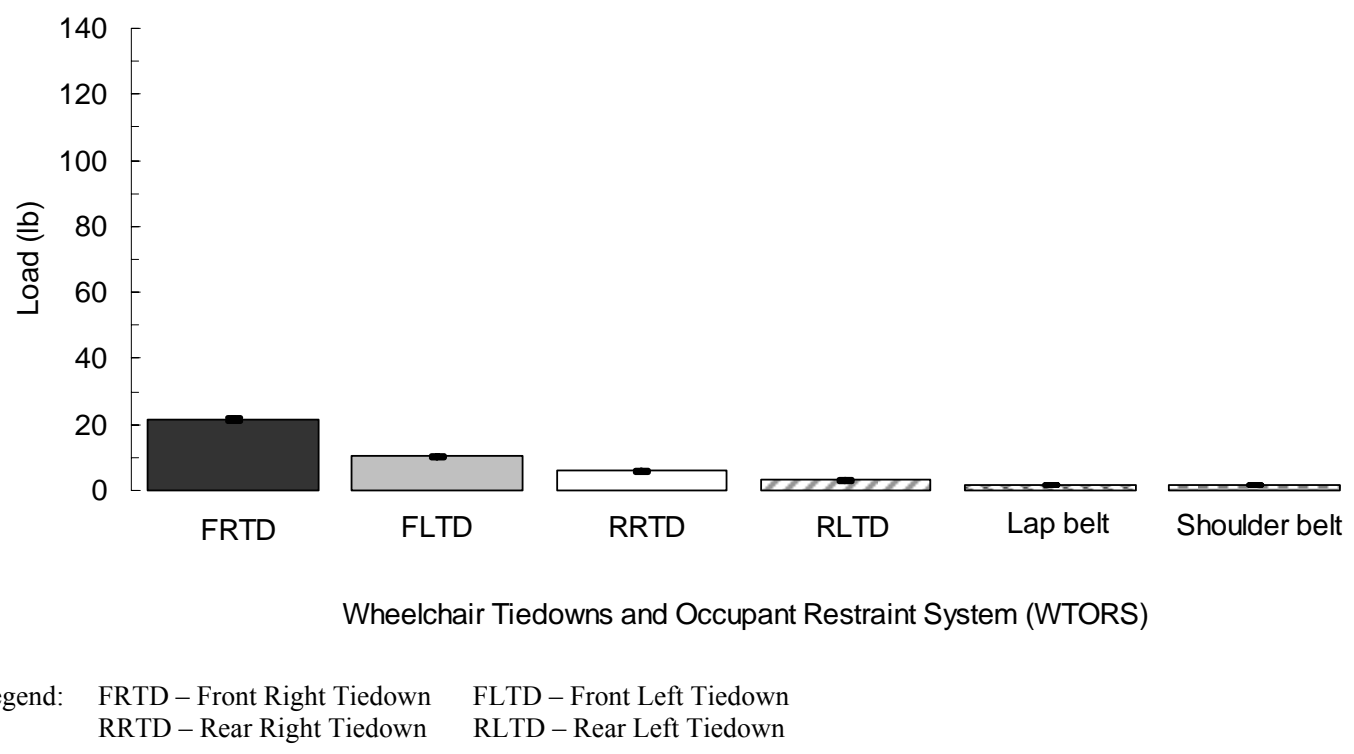

Figure 7. Mean peak WTORS loading during LATV emergency acceleration $(\mathrm{n}=3$, error bars indicate $95 \%$ confidence intervals). 


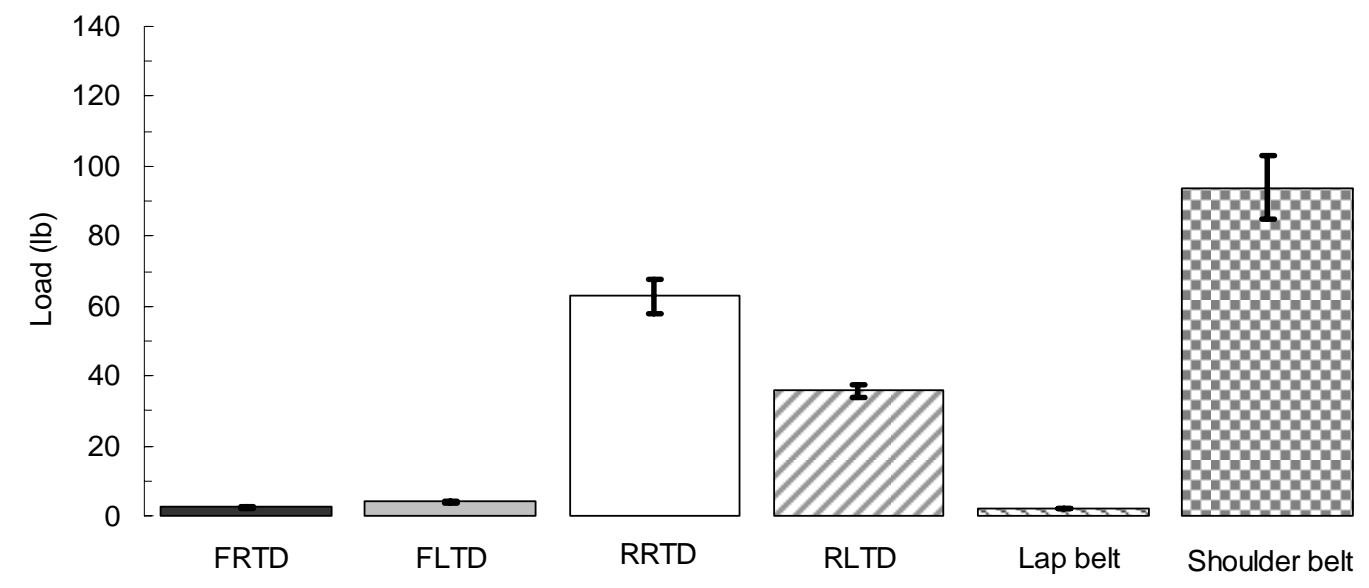

Wheelchair Tiedowns and Occupant Restraint System (WTORS)

Legend: $\quad$ FRTD - Front Right Tiedown $\quad$ FLTD - Front Left Tiedown RRTD - Rear Right Tiedown RLTD - Rear Left Tiedown

Figure 8. Mean peak WTORS loading during LATV emergency braking $(\mathrm{n}=3$, error bars indicate $95 \%$ confidence intervals).

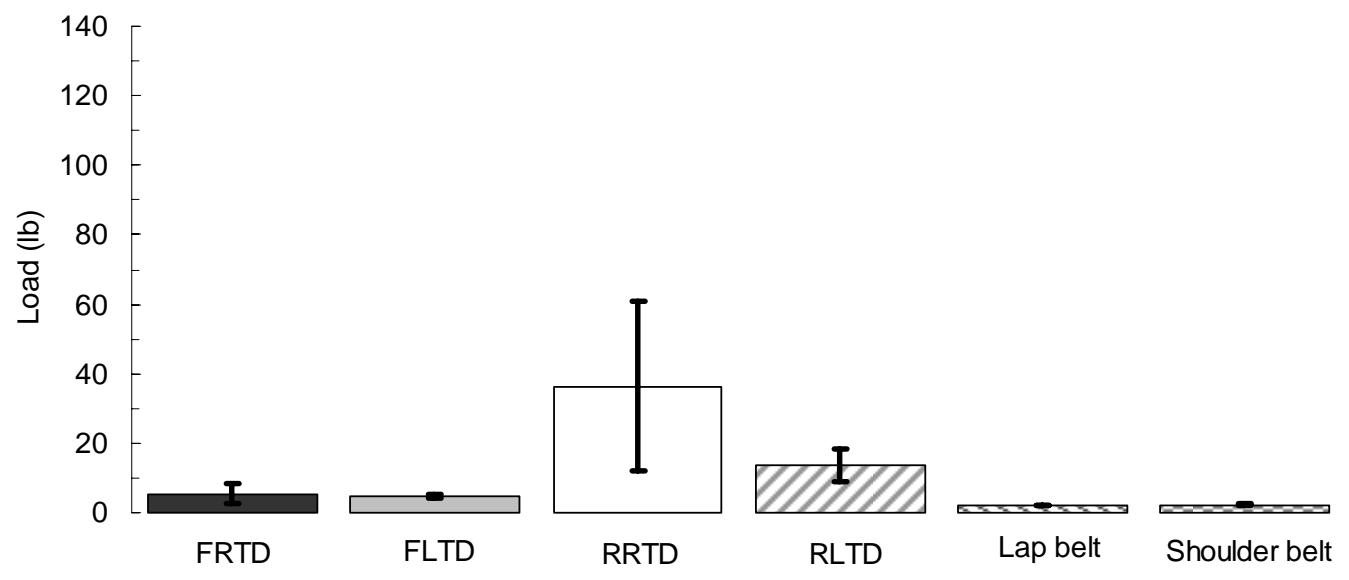

Wheelchair Tiedowns and Occupant Restraint System (WTORS)

$\begin{array}{lll}\text { Legend: } & \text { FRTD }- \text { Front Right Tiedown } & \text { FLTD }- \text { Front Left Tiedown } \\ & \text { RRTD - Rear Right Tiedown } & \text { RLTD }- \text { Rear Left Tiedown }\end{array}$

Figure 9. Mean peak WTORS loading during LATV emergency left turns ( $\mathrm{n}=3$, error bars indicate $95 \%$ confidence intervals). 


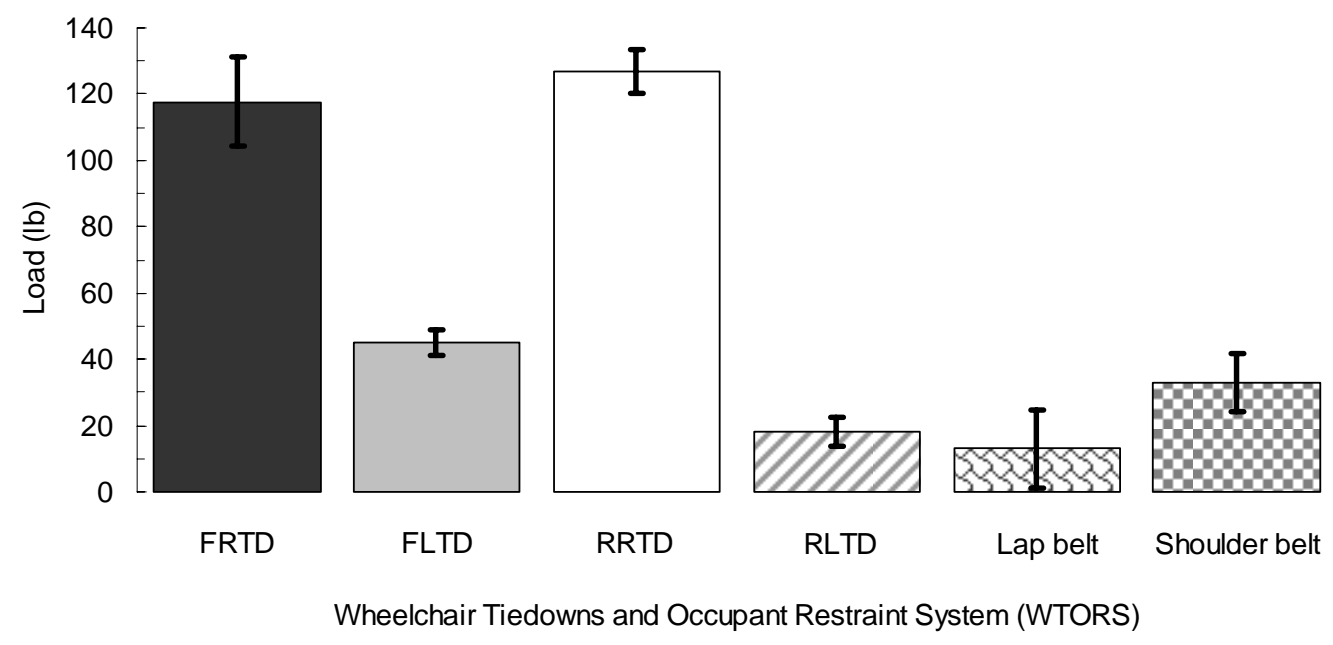

$\begin{array}{lll}\text { Legend: } & \text { FRTD - Front Right Tiedown } & \text { FLTD - Front Left Tiedown } \\ & \text { RRTD - Rear Right Tiedown } & \text { RLTD - Rear Left Tiedown }\end{array}$

Figure 10. Mean peak WTORS loading during LATV emergency right turns ( $\mathrm{n}=3$, error bars indicate $95 \%$ confidence intervals).

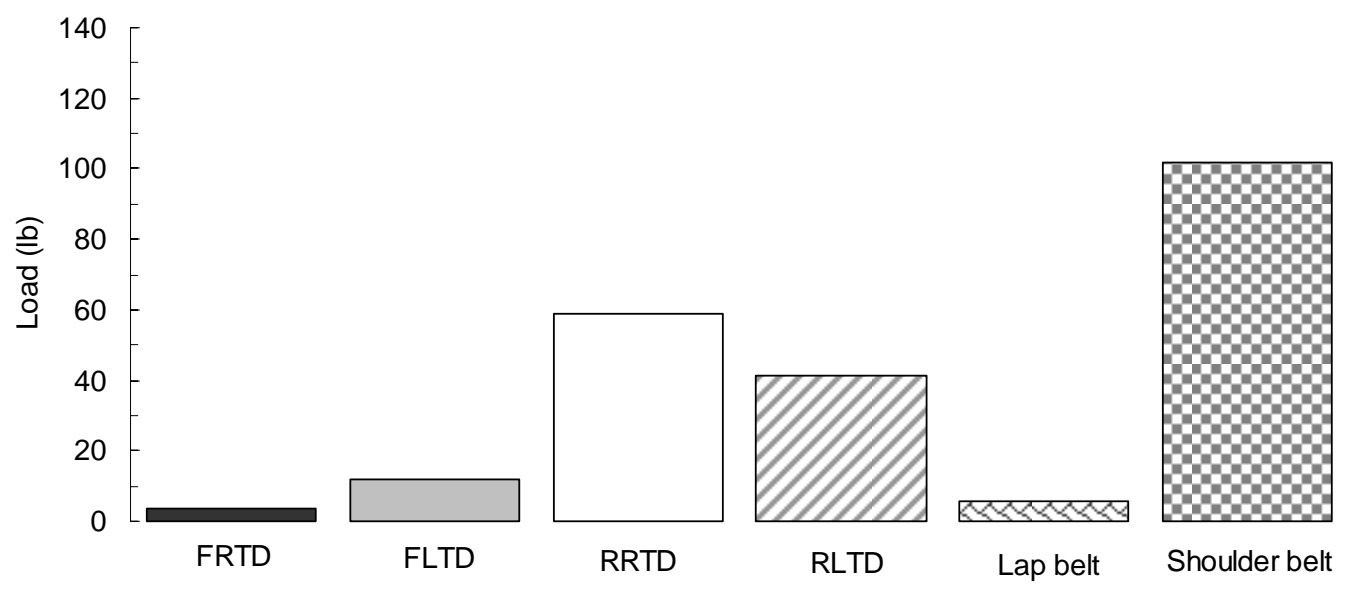

Wheelchair Tiedowns and Occupant Restraint System (WTORS)

Legend: $\quad$ FRTD - Front Right Tiedown $\quad$ FLTD - Front Left Tiedown RRTD - Rear Right Tiedown RLTD - Rear Left Tiedown

Figure 11. Mean peak WTORS loading for LATV emergency braking/left-turn maneuvers $(n=2$, error bars not shown for sample sizes $n<3)$. 


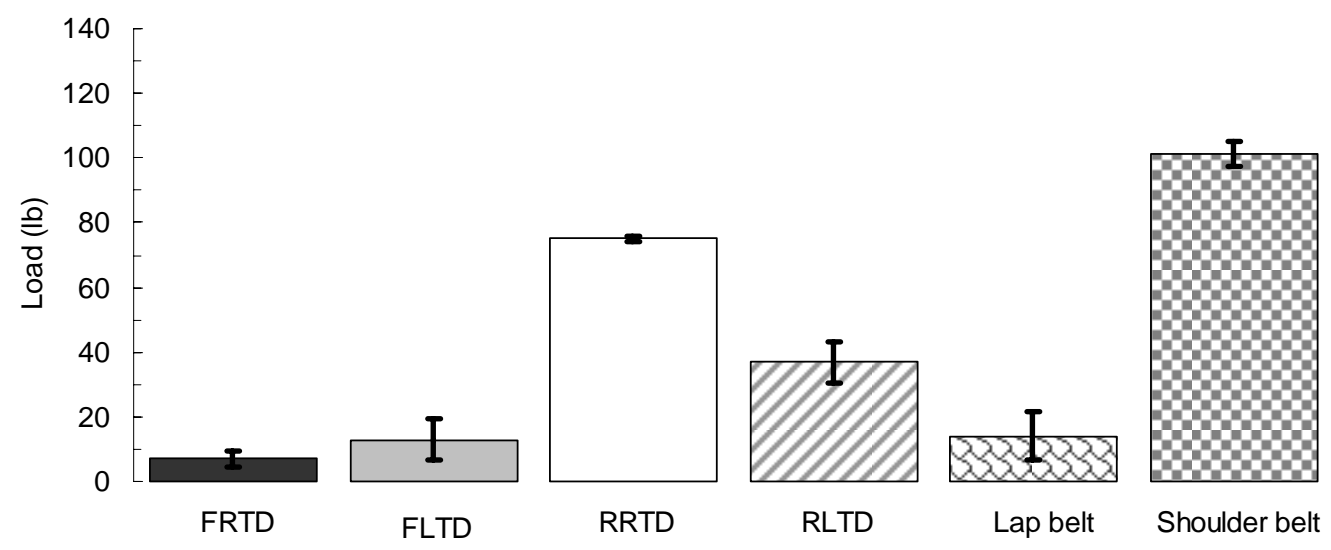

Wheelchair Tiedowns and Occupant Restraint System (WTORS)

Legend: $\quad$ FRTD - Front Right Tiedown $\quad$ FLTD - Front Left Tiedown

RRTD - Rear Right Tiedown $\quad$ RLTD - Rear Left Tiedown

Figure 12. Mean peak WTORS loading for LATV emergency braking/right-turn maneuvers ( $\mathrm{n}=3$, error bars indicate $95 \%$ confidence intervals).

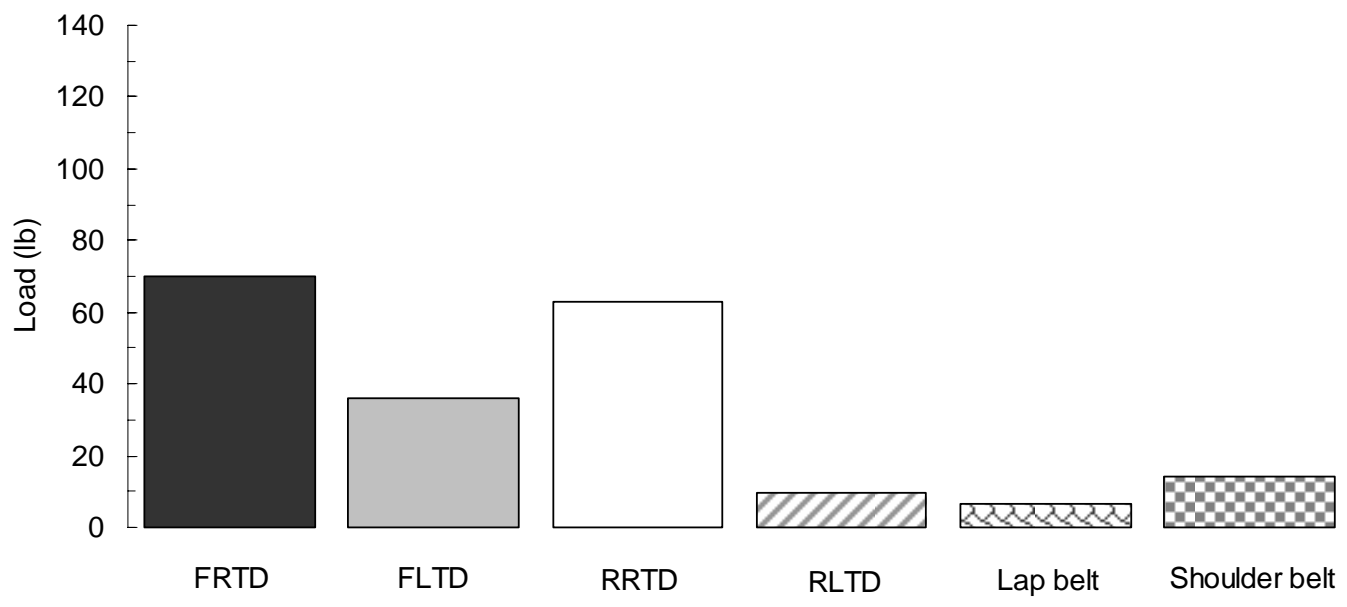

Wheelchair Tiedowns and Occupant Restraint System (WTORS)

Legend: $\quad$ FRTD - Front Right Tiedown $\quad$ FLTD - Front Left Tiedown RRTD - Rear Right Tiedown $\quad$ RLTD - Rear Left Tiedown

Figure 13. Mean peak WTORS loading for LATV emergency lane change maneuvers $(\mathrm{n}=2$, error bars not shown for sample sizes $\mathrm{n}<3$ ). 
Tables 3-5 show outcome measures for all LATV emergency maneuvers across all three WTORS configurations. Table 3 summarizes outcome measures for LATV emergency maneuvers with proper WTORS use (configuration C). The wheelchair made benign contact with securement station right side barrier (folded-seat under window) during left turn, braking/left-turn, and lane change maneuvers. The ATD remained seated in the wheelchair during all LATV emergency maneuvers with WTORS configuration C.

Table 4 depicts outcome measures for LATV emergency maneuvers with WTORS misuse (configuration B), when the lap belt was routed around the wheelchair seatback in an attempt to secure the wheelchair. For WTORS configuration B, the highest overall peak LB load was $174.1 \mathrm{lb}$ and occurred during a right turn. The wheelchair and/or ATD impacted the LATV interior in nine out of eleven (82\%) maneuvers, while the ATD was ejected from the wheelchair in seven out of eleven (64\%) maneuvers. ATD ejection occurred during all maneuvers, except for acceleration. The wheelchair tipped over in four of eleven (36\%) maneuvers.

Table 5 depicts outcome measures for LATV emergency maneuvers with WTORS disuse (configuration A). For WTORS configuration A, the wheelchair and/or ATD impacted the LATV interior in six out of seven (87\%) maneuvers, while the ATD was ejected from the wheelchair in four out of seven (57\%) maneuvers. ATD ejection occurred during braking, left turn, right turn, braking/left-turn, and braking/right-turn maneuvers. 


\begin{tabular}{|c|c|c|c|c|c|c|c|c|c|c|c|c|c|c|c|c|}
\hline \multirow[b]{2}{*}{ Trial } & \multirow[b]{2}{*}{ Maneuver } & \multicolumn{3}{|c|}{ Peak LATV accelerations } & \multirow{2}{*}{\begin{tabular}{|l|}
$\begin{array}{c}\text { Peak WC } \\
\text { acceleration }\end{array}$ \\
$\begin{array}{c}\text { Peak WC resultant } \\
\text { acceleration (g) }\end{array}$ \\
\end{tabular}} & \multicolumn{2}{|c|}{ Peak ATD accelerations } & \multicolumn{6}{|c|}{ Peak WTORS Loading } & \multicolumn{2}{|c|}{$\begin{array}{l}\text { Gross WC and ATD } \\
\text { dynamics }\end{array}$} & \multirow[b]{2}{*}{ Notes } \\
\hline & & $\begin{array}{c}\text { Peak LATV } \\
\text { fore/aft } \\
\text { acceleration }(g\end{array}$ & $\begin{array}{c}\text { Peak LATV } \\
\text { latreal } \\
\text { acceleration (g) }\end{array}$ & $\begin{array}{c}\text { Peak LATV } \\
\text { resultant } \\
\text { acceleration (g) }\end{array}$ & & \begin{tabular}{|c}
$\begin{array}{c}\text { Peak ATD } \\
\text { chest resultat } \\
\text { acceleration (g) }\end{array}$ \\
at
\end{tabular} & \begin{tabular}{|c}
$\begin{array}{c}\text { Peak ATD } \\
\text { head resultatt } \\
\text { acceleration (g) }\end{array}$ \\
(9)
\end{tabular} & \begin{tabular}{|c}
$\begin{array}{c}\text { Peak FRTD } \\
\text { Loading } \\
\text { (b) }\end{array}$ \\
\end{tabular} & \begin{tabular}{|c}
$\begin{array}{c}\text { Peak FLTD } \\
\text { Loading } \\
\text { (lb) }\end{array}$ \\
\end{tabular} & \begin{tabular}{|c|} 
Peak \\
RRTD \\
Loading \\
(b)
\end{tabular} & $\begin{array}{c}\text { Peak } \\
\text { RLTD } \\
\text { Loading } \\
\text { (b) }\end{array}$ & $\begin{array}{l}\text { Peak LB } \\
\text { Loading } \\
\text { (b) }\end{array}$ & $\begin{array}{l}\text { Peak SB } \\
\text { Loading } \\
\text { (lb) }\end{array}$ & \begin{tabular}{|c|} 
wC contact \\
sec station \\
boundries \\
(YN)?
\end{tabular} & $\begin{array}{c}\text { ATD ejected } \\
\text { from WC } \\
(\mathrm{YN}) ?\end{array}$ & \\
\hline 401 & Acceleration & 0.26 & 0.07 & 0.27 & 0.25 & 0.32 & 0.35 & 21.1 & 10.1 & 6.2 & 3.6 & 1.8 & 1.6 & $\mathrm{~N}$ & $\mathrm{~N}$ & WC remains secured ATD remains restrained \\
\hline 402 & Acceleration & 0.28 & 0.06 & 0.29 & 0.32 & 0.33 & 0.33 & 20.6 & 10.1 & 5.7 & 2.9 & 1.8 & 1.5 & $\mathrm{~N}$ & $\mathrm{~N}$ & WC remains secured ATD remains restrained \\
\hline 403 & Acceleration & 0.30 & 0.05 & 0.30 & 0.26 & 0.31 & 0.33 & 22.6 & 10.8 & 5.8 & 2.7 & 1.9 & 1.8 & $\mathrm{~N}$ & $\mathrm{~N}$ & WC remains secured ATD remains restrained \\
\hline 419 & Braking & 0.89 & 0.07 & 0.89 & 0.85 & 3.02 & 4.55 & 2.0 & 3.7 & 68.7 & 37.6 & 1.9 & 105.6 & $\mathrm{~N}$ & $\mathrm{~N}$ & WC remains secured ATD remains restrained \\
\hline 420 & Braking & 0.89 & 0.07 & 0.89 & 0.82 & 2.21 & 3.62 & 2.5 & 4.0 & 63.9 & 32.7 & 1.9 & 80.9 & $\mathrm{~N}$ & $\mathrm{~N}$ & WC remains secured ATD remains restrained \\
\hline 421 & Braking & 0.80 & 0.12 & 0.81 & 0.95 & 2.44 & 3.81 & 2.7 & 4.5 & 55.5 & 36.8 & 2.0 & 95.2 & $\mathrm{~N}$ & $\mathrm{~N}$ & WC remains secured ATD remains restrained \\
\hline 404 & Left Turn & 0.13 & 0.64 & 0.65 & 1.11 & 1.03 & 1.26 & 11.2 & 3.5 & 10.0 & 11.0 & 1.8 & 1.7 & $\mathrm{Y}$ & $\mathrm{N}$ & $\begin{array}{l}\text { WC remains secured ATD remains restrained, WC makes contact with right securement } \\
\text { station barrier (folding seat under window) }\end{array}$ \\
\hline 408 & Left Turn & 0.10 & 0.62 & 0.63 & 0.93 & 1.06 & 1.17 & 2.6 & 5.5 & 20.1 & 8.0 & 1.9 & 2.1 & $\mathrm{Y}$ & $\mathrm{N}$ & $\begin{array}{l}\text { WC remains secured ATD remains restrained, WC makes contact with right securement } \\
\text { station barrier (folding seat under window) }\end{array}$ \\
\hline 410 & Left Turn & 0.32 & 0.71 & 0.78 & 1.08 & 1.58 & 1.91 & 2.4 & 4.8 & 79.0 & 21.5 & 2.0 & 2.9 & $\mathrm{Y}$ & $\mathrm{N}$ & $\begin{array}{l}\text { WC remains secured AAD remains restrained, WC makes contact with right securement } \\
\text { station barrier (folding seat under window). LATV Operator applied brake slightly durin } \\
\text { maneuver to avoid camera man. }\end{array}$ \\
\hline 405 & Right Turn & 0.21 & 0.68 & 0.71 & 1.16 & 1.58 & 1.96 & 93.3 & 51.5 & 120.0 & 11.2 & 2.0 & 18.1 & $\mathrm{~N}$ & $\mathrm{~N}$ & WC remains secured ATD remains restrained \\
\hline 407 & Right Turn & 0.24 & 0.64 & 0.68 & 1.16 & 2.84 & 3.08 & 124.5 & 40.6 & 138.4 & 24.3 & 3.3 & 36.2 & $\mathrm{~N}$ & $\mathrm{~N}$ & WC remains secured ATD remains restrained \\
\hline 409 & Right Turn & 0.25 & 0.73 & 0.77 & 1.25 & 3.06 & 3.36 & 135.5 & 43.0 & 122.1 & 18.9 & 34.0 & 44.4 & $\mathrm{~N}$ & $\mathrm{~N}$ & WC remains secured ATD remains restrained \\
\hline 414 & \begin{tabular}{|c|}
$\begin{array}{c}\text { Braking/Left } \\
\text { Turn }\end{array}$ \\
\end{tabular} & 0.88 & 0.42 & 0.98 & 1.26 & 2.86 & 4.60 & 2.6 & 20.5 & 73.2 & 44.4 & 9.8 & 96.3 & $\mathrm{Y}$ & $\mathrm{N}$ & $\begin{array}{l}\text { WC remains secured ATD remains restrained, WC makes contact with right securement } \\
\text { station barrier f(folding seat under window) }\end{array}$ \\
\hline 418 & \begin{tabular}{|c|}
$\begin{array}{c}\text { Braking / Left } \\
\text { Turn }\end{array}$ \\
\end{tabular} & 0.87 & 0.35 & 0.94 & 1.33 & 2.65 & 3.88 & 4.3 & 3.3 & 44.4 & 38.4 & 1.9 & 107.2 & $\mathrm{Y}$ & $\mathrm{N}$ & 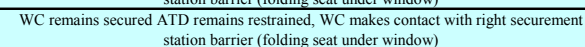 \\
\hline 413 & $\begin{array}{c}\text { Braking / Right } \\
\text { Turn }\end{array}$ & 0.77 & 0.57 & 0.96 & 1.22 & 2.82 & 4.65 & 10.1 & 11.7 & 75.7 & 38.6 & 16.9 & 99.9 & $\mathrm{~N}$ & $\mathrm{~N}$ & 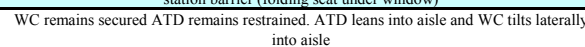 \\
\hline 415 & \begin{tabular}{c|c}
$\begin{array}{c}\text { Braking } / \text { Right } \\
\text { Turn }\end{array}$ \\
\end{tabular} & 0.87 & 0.61 & 1.06 & 1.06 & 3.15 & 4.57 & 8.4 & 22.2 & 75.9 & 44.9 & 22.4 & 107.2 & $\mathrm{~N}$ & $\mathrm{~N}$ & $\begin{array}{l}\text { WC remains secured ATD remains restraindised ATD leans into aisle and WC tilts laterally } \\
\text { into aisle }\end{array}$ \\
\hline 417 & \begin{tabular}{|c|c|c|c|}
$\begin{array}{c}\text { Braking } / \text { Right } \\
\text { Turn }\end{array}$ \\
\end{tabular} & 0.74 & 0.54 & 0.92 & 1.04 & 2.43 & 3.74 & 2.5 & 4.6 & 73.6 & 27.4 & 2.3 & 97.1 & $\mathrm{~N}$ & $\mathrm{~N}$ & $\begin{array}{l}\text { WC remains secured ATD remains restraindadi ATD leans into aisle and WC tilts laterally } \\
\text { into aisle }\end{array}$ \\
\hline 411 & Lane Changes & 0.25 & 0.51 & 0.57 & 0.90 & 1.33 & 1.86 & 44.4 & 18.8 & 43.9 & 5.4 & 7.3 & 14.3 & $\mathrm{Y}$ & $\mathrm{N}$ & $\begin{array}{l}\text { WC remains secured ATD remains restrained. ATD leans into aisle and WC tilts lateralllly } \\
\text { into aisle and WC makes contact with right securement station barrier (folding seat under } \\
\text { window). }\end{array}$ \\
\hline 412 & Lane Changes & 0.28 & 0.57 & 0.64 & 1.04 & 2.15 & 2.88 & 95.5 & 53.0 & 82.0 & 14.3 & 5.4 & 14.3 & $\mathrm{Y}$ & $\mathrm{N}$ & $\begin{array}{l}\text { WC remains secured ATD remains restrained. ATD leans into aisle and WC tilts laterallle } \\
\text { into aisle and WC makes contact with hights securement station barrier foldding seat under } \\
\text { window). }\end{array}$ \\
\hline
\end{tabular}

Highest peak outcome measure value

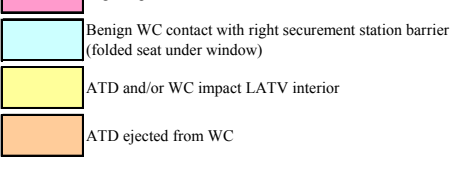

Table 3. Outcome measures recorded during LATV emergency driving maneuvers with proper WTORS use (configuration C). 


\begin{tabular}{|c|c|c|c|c|c|c|c|c|c|c|c|c|c|c|c|c|}
\hline \multirow[b]{2}{*}{ Trial } & \multirow[b]{2}{*}{ Maneuver } & \multicolumn{3}{|c|}{ Peak LATV accelerations } & \multirow{2}{*}{\begin{tabular}{|c|} 
Peak WC \\
acceleration
\end{tabular}} & \multicolumn{2}{|c|}{ Peak ATD accelerations } & \multicolumn{6}{|c|}{ Peak WTORS Loading } & \multicolumn{2}{|c|}{$\begin{array}{c}\text { Gross WC and ATD } \\
\text { dynamics }\end{array}$} & \multirow[b]{2}{*}{ Notes } \\
\hline & & \begin{tabular}{|l} 
Peak LATV \\
fore/at \\
acceleration $(\mathrm{g})$
\end{tabular} & \begin{tabular}{|c|c} 
Peak LATV \\
lateral \\
acceleration (g)
\end{tabular} & $\begin{array}{c}\text { Peak LATV } \\
\text { resultant } \\
\text { acceleration }(\mathrm{gg}\end{array}$ & & $\left|\begin{array}{c}\text { Peak ATD } \\
\text { chest resultant } \\
\text { acceleration (g) }\end{array}\right|$ & $\left|\begin{array}{c}\text { Peak ATD } \\
\text { head resultant } \\
\text { acceleration }(\mathrm{g}\end{array}\right|$ P & $\begin{array}{l}\text { Peak FRTD } \\
\text { Loading } \\
\text { (b) }\end{array}$ & $\left|\begin{array}{c}\text { Peak FLTD } \\
\text { Loading } \\
\text { (lb) }\end{array}\right|$ & \begin{tabular}{|c|} 
Peak \\
RRTD \\
Loading \\
(b)
\end{tabular} & $\begin{array}{c}\text { Peak } \\
\text { RLTD } \\
\text { Loading } \\
\text { (b) }\end{array}$ & $\begin{array}{l}\text { Peak LB } \\
\text { Loading } \\
\text { (lb) }\end{array}$ & $\begin{array}{l}\text { Peak SB } \\
\text { Loading } \\
\text { (lb) }\end{array}$ & $\begin{array}{c}\text { WC contact } \\
\text { sec station } \\
\text { boundries } \\
\text { (YN)? }\end{array}$ & $\begin{array}{c}\text { ATD ejected } \\
\text { from WC } \\
(\mathrm{YN}) ?\end{array}$ & \\
\hline 501 & Acceleration & 0.27 & 0.05 & 0.28 & $\mathrm{EX}$ & $\mathrm{EX}$ & $\mathrm{EX}$ & 0.0 & 0.0 & 0.0 & 0.0 & N/A & 0.0 & $\mathrm{Y}$ & $\mathrm{N}$ & WC tipped over backwards, WC seatback and ATD head and back strike rear securement \\
\hline 502 & Acceleration & 0.28 & 0.07 & 0.29 & $\mathrm{EX}$ & $\mathrm{EX}$ & $\mathrm{EX}$ & 0.0 & 0.0 & 0.0 & 0.0 & 1.9 & 0.0 & $\mathrm{Y}$ & $\mathrm{N}$ & $\begin{array}{l}\text { WC tipped over backwards, WC seatback and ATD head and back strike rear securement } \\
\text { station barrier }\end{array}$ \\
\hline 503 & Acceleration & 0.27 & 0.07 & 0.28 & $\mathrm{EX}$ & $\mathrm{EX}$ & $\mathrm{EX}$ & 0.0 & 0.0 & 0.0 & 0.0 & 4.0 & 0.0 & $\mathrm{Y}$ & $\mathrm{N}$ & $\begin{array}{l}\text { WC tipped over backwards, WC seatback and ATD head and back strike rear securement } \\
\text { station barrier }\end{array}$ \\
\hline 509 & Braking & 0.83 & 0.10 & 0.84 & 1.25 & $\mathrm{EX}$ & $\mathrm{EX}$ & 0 & 00 & 00 & 00 & 55.3 & 00 & $\mathrm{~N}$ & $\mathrm{Y}$ & ATD ejected to the front of LATV. WC remains stationary. \\
\hline 504 & Left Turn & 0.26 & 0.64 & 0.69 & 1.10 & 1.35 & 2.04 & 0.0 & 0.0 & 0.0 & 0.0 & 0.5 & 0.0 & $\mathrm{Y}$ & $\mathrm{N}$ & WC makes contact with securement station right side barrier (folded seat under window) \\
\hline 511 & Left Turn & 0.23 & 0.70 & 0.74 & 1.18 & $\mathrm{EX}$ & $\mathrm{EX}$ & 00 & 00 & 00 & 00 & 4.6 & 00 & $\mathrm{Y}$ & $\mathrm{Y}$ & $\begin{array}{l}\text { ATD ejected to the front of LATV. WC makes contact with securement station right side } \\
\text { barrier (folded seat under window) }\end{array}$ \\
\hline 505 & Right Turn & 0.21 & 0.96 & 0.98 & $\mathrm{EX}$ & $\mathrm{EX}$ & $\mathrm{EX}$ & 20 & 20 & 20 & 20 & 0.3 & 0 & $\overline{\mathrm{Y}}$ & $\mathrm{Y}$ & $\begin{array}{l}\text { ATD ejected laterally into aisle. WC and ATD strike camera mounted in place of left side } \\
\text { securement station. }\end{array}$ \\
\hline 510 & Right Turn & 0.20 & 0.83 & 0.85 & EX & $\mathrm{EX}$ & EX & 0 & 00 & 00 & 00 & 174.1 & 0 & $\mathrm{Y}$ & $\mathrm{Y}$ & $\begin{array}{l}\text { ATD ejected laterally into aisle. WC and ATD strike camera mounted in place of left side } \\
\text { securement station. }\end{array}$ \\
\hline 508 & \begin{tabular}{|c|} 
Braking / Left \\
Turn
\end{tabular} & 1.10 & 0.61 & 1.26 & 1.59 & $\mathrm{EX}$ & $\mathrm{EX}$ & 0 & 00 & 0 & 00 & 54.3 & 0.0 & $\mathrm{Y}$ & $\mathrm{Y}$ & $\begin{array}{l}\text { ATD ejected to the front of LATV. WC makes contact with securement station right side } \\
\text { barrier (folded seat under window) }\end{array}$ \\
\hline 507 & \begin{tabular}{|c|c|c|}
$\begin{array}{c}\text { Braking/Right } \\
\text { Tum }\end{array}$ \\
\end{tabular} & 0.86 & 0.38 & 0.94 & 1.91 & $\mathrm{EX}$ & $\mathrm{EX}$ & 00 & 00 & 00 & 0.0 & 75.6 & 00 & $\mathrm{~N}$ & $\mathrm{Y}$ & ATD ejected to the front of LATV. WC remains stationary. \\
\hline 506 & Lane Changes & 0.17 & 0.52 & 0.55 & $\mathrm{EX}$ & EX & EX & 00 & 00 & 0.0 & 000 & 62.0 & & $\mathrm{Y}$ & $\mathrm{Y}$ & WC tips over laterally into aisle, ATD ejected from WC. \\
\hline
\end{tabular}

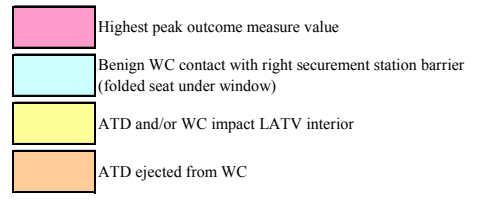

EX-Exceeded accelerometer range

Table 4. Outcome measures recorded during LATV emergency driving maneuvers with WTORS misuse (configuration B). 


\begin{tabular}{|c|c|c|c|c|c|c|c|c|c|c|c|c|c|c|c|c|}
\hline \multirow[b]{2}{*}{ Trial } & \multirow[b]{2}{*}{ Maneuver } & \multicolumn{3}{|c|}{ Peak LATV accelerations } & \multirow{2}{*}{\begin{tabular}{|c|}
$\begin{array}{c}\text { Peak WC } \\
\text { acceleration }\end{array}$ \\
$\begin{array}{c}\text { Peak WC } \\
\text { resultant } \\
\text { acceleration }(g)\end{array}$ \\
\end{tabular}} & \multicolumn{2}{|c|}{ Peak ATD accelerations } & \multicolumn{6}{|c|}{ Peak WTORS Loading } & \multicolumn{2}{|c|}{$\begin{array}{l}\text { Gross WC and ATD } \\
\text { dynamics }\end{array}$} & \multirow[b]{2}{*}{ Notes } \\
\hline & & $\begin{array}{l}\text { Peak LATV } \\
\text { fore aft } \\
\text { icceleration }(g)\end{array}$ & $\begin{array}{c}\text { Peak LATV } \\
\text { latral } \\
\text { acceleration (g) }\end{array}$ & $\begin{array}{c}\text { Peak LATV } \\
\text { resultant } \\
\text { acceleration }(\mathrm{g})\end{array}$ & & $\begin{array}{c}\text { Peak ATD } \\
\text { chest resultant } \\
\text { acceleration }(\mathrm{g}\end{array}$ & $\begin{array}{l}\begin{array}{c}\text { Peak ATD } \\
\text { head resultat } \\
\text { acceleration (g) }\end{array} \\
\text { (g) }\end{array}$ & $\begin{array}{c}\text { Peak FRTD } \\
\text { Loading } \\
\text { (b) }\end{array}$ & $\mid \begin{array}{c}\text { Peak FLTD } \\
\text { Loading } \\
\text { (lb) }\end{array}$ & \begin{tabular}{|c} 
Peak \\
RRTD \\
Loading \\
(lb)
\end{tabular} & $\begin{array}{c}\text { Peak } \\
\text { RLTD } \\
\text { Loading } \\
\text { (b) }\end{array}$ & $\begin{array}{l}\text { Peak LB } \\
\text { Loading } \\
\text { (lb) }\end{array}$ & \begin{tabular}{|l} 
Peak SB \\
Loading \\
(lb)
\end{tabular} & $\begin{array}{c}\text { WC contact } \\
\text { sec station } \\
\text { boundries } \\
(\mathrm{Y} / \mathrm{N}) ?\end{array}$ & $\begin{array}{c}\text { ATD ejected } \\
\text { from WC } \\
\text { (YN)? }\end{array}$ & \\
\hline 601 & Acceleration & 0.27 & 0.07 & 0.28 & $\mathrm{EX}$ & 0.98 & 1.38 & 0.0 & 0.0 & 0.0 & 0.0 & 0.0 & 0.0 & $\mathrm{Y}$ & $\mathrm{N}$ & WC rotates laterally into aisle and rolls reward. WC strikes rear securement station barrier \\
\hline 607 & Braking & 0.92 & 0.09 & 0.92 & $\mathrm{EX}$ & $\mathrm{EX}$ & $\mathrm{EX}$ & +0 & 00 & 00 & +0 & 00 & 00 & $\mathrm{Y}$ & $\mathrm{Y}$ & ATD ejected to the front of LATV. WC is projected into the aisle. \\
\hline 603 & Left Turn & 0.16 & 0.79 & 0.81 & 1.22 & 1.86 & 1.19 & 0.0 & 0.0 & 0.0 & 0.0 & 0.0 & 0.0 & $\bar{Y}$ & $\mathrm{~N}$ & $\begin{array}{l}\text { WC and ATD make contact with securement station right side barrier (folded seat under } \\
\text { window) }\end{array}$ \\
\hline 602 & Right Turn & 0.25 & 0.98 & 1.01 & EX & $\mathrm{EX}$ & $\mathrm{EX}$ & 00 & 00 & 00 & 0 & 00 & 00 & $\bar{Y}$ & $\mathrm{Y}$ & $\begin{array}{l}\text { ATD ejected laterally into aisle. WC and ATD strike camera mounted in place of leff side } \\
\text { securement station. }\end{array}$ \\
\hline 605 & \begin{tabular}{|c|c|c|c|c|} 
Braking/Left \\
Turn
\end{tabular} & 0.87 & 0.44 & 0.97 & $\mathrm{EX}$ & $\mathrm{EX}$ & $\mathrm{EX}$ & 20 & 20 & 20 & 20 & 20 & 20 & $\mathrm{Y}$ & $\mathrm{Y}$ & $\begin{array}{l}\text { ATD ejected to the front of LATV. WC makes contact with securement station right side } \\
\text { barrier (folded seat under window) and is then projected into the aisle. }\end{array}$ \\
\hline 606 & \begin{tabular}{|c|c|c|c|} 
raking/ Right \\
Tum
\end{tabular} & 0.89 & 0.41 & 0.98 & $\mathrm{EX}$ & EX & $\mathrm{EX}$ & 0 & 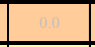 & 20 & 30 & 0 & 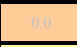 & $\mathrm{Y}$ & $\mathrm{Y}$ & ATD ejected to the front of LATV. WC is projected into the aisle. \\
\hline 604 & Lane Changes & 0.22 & 0.63 & 0.67 & $\mathrm{EX}$ & EX & EX & 0.0 & 0.0 & 0.0 & 0.0 & 0.0 & 0.0 & $\mathrm{Y}$ & $\mathrm{N}$ & $\begin{array}{l}\text { WC rotates laterally into aisle and rolls reward. WC and ATD strike right-side sec station } \\
\text { barrier (folded seat under window) and securement station across the aisle }\end{array}$ \\
\hline
\end{tabular}

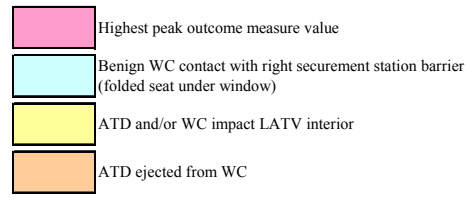

WC rotates laterally into aisle and rolls reward. WC and ATD strike right-side sec
barrier (folded seat under window) and securement station across the aisle

Table 5. Outcome measures recorded during LATV emergency driving maneuvers with WTORS disuse (configuration A). 


\section{Discussion and Conclusions}

As expected, LATV accelerations associated with emergency maneuvers in this study were higher than those reported in normal driving. We found mean peak LATV accelerations in normal driving of $0.21 \mathrm{~g}$ during braking maneuvers, $0.24 \mathrm{~g}$ during acceleration maneuvers, $0.25 \mathrm{~g}$ during left turns, and $0.37 \mathrm{~g}$ during right turns. Zaworski, et al. ${ }^{11}$ reported peak LATV accelerations for various normal driving maneuvers on multiple routes and for a variety of LATV designs. In their study, they reported that LATV forward accelerations (accelerations from standstill) ranged from $0 \mathrm{~g}$ to $0.20 \mathrm{~g}$, left turn and right turn accelerations ranged from $0.05 \mathrm{~g}$ to $0.40 \mathrm{~g}$, while LATV accelerations for stopping maneuvers ranged from $0.05 \mathrm{~g}$ to $0.40 \mathrm{~g}$. Another study by van Roosmalen, et al. ${ }^{29}$ reported maximum LATV accelerations of $0.60 \mathrm{~g}$ during normal driving maneuvers; a range of $0.56 \mathrm{~g}$ to $0.75 \mathrm{~g}$ (mean $0.64 \mathrm{~g}$, standard deviation, SD $0.1 \mathrm{~g}$ ) for braking maneuvers, and $0.38 \mathrm{~g}$ to $0.56 \mathrm{~g}$ (mean $0.47 \mathrm{~g}$, SD $0.1 \mathrm{~g}$ ) for turning maneuvers. While developing a platform system to assess wheelchair passenger postural response to vehicle perturbations in normal driving, Kamper, et al. ${ }^{26}$ used vehicle accelerations of $0.2 \mathrm{~g}$ and $0.4 \mathrm{~g}$ to investigate wheelchair passenger postural stability. Sprigle and Linden ${ }^{24}$ studied accelerations wheelchair passengers may experience in modified van and reported vehicle accelerations of $0.084-0.250 \mathrm{~g}$ during acceleration, $0.063-0.719 \mathrm{~g}$ during braking, and

0.133-0.701g during turning. In another study investigating vehicle accelerations experienced by wheelchair passengers, Linden and Sprigle ${ }^{23}$ reported mean total vehicle accelerations of $0.5 \mathrm{~g}$ and less in a modified van under various normal driving maneuvers and across various positions in the vehicle (driver, front, and rear). The mean peak LATV resultant accelerations during emergency driving in our study were higher than those in 
reported in normal driving (acceleration: mean $0.28 \mathrm{~g}, 95 \%$ confidence interval, CI $0.01 \mathrm{~g}$; braking: mean $0.87 \mathrm{~g}$, CI $0.04 \mathrm{~g}$; left turn: mean $0.72 \mathrm{~g}$, CI $0.05 \mathrm{~g}$; right turn: $0.84 \mathrm{~g}$, CI $0.10 \mathrm{~g}$; braking/left-turn: mean $1.04 \mathrm{~g}$, CI $0.13 \mathrm{~g}$; braking/right-turn: mean $0.97 \mathrm{~g}$, CI $0.04 \mathrm{~g}$; lane changes: mean $0.61 \mathrm{~g}$, CI $0.05 \mathrm{~g}$ ). Higher LATV accelerations during emergency vs. normal driving were expected, as Zaworski, et al. ${ }^{11}$ also reported substantially higher LATV accelerations associated with emergency driving compared to normal driving. The emergency driving mean peak LATV accelerations for the LATV fleet in the Zaworski, et al. ${ }^{11}$ study were $0.79 \mathrm{~g}$ during braking, $0.38 \mathrm{~g}$ during acceleration, $0.38 \mathrm{~g}$ during left turns, and $0.42 \mathrm{~g}$ during right turns. The LATV accelerations in our study were higher than those found by Zaworski, et al. ${ }^{11}$, with the exception of LATV acceleration maneuvers. This difference may be because the LATV in our study was powered by a hybrid (diesel-electric) engine, which may not accelerate as quickly as an LATV powered by a standard diesel engine, such as those used in the Zaworski, et al. ${ }^{11}$ study. Other factors that may have contributed to the difference in LATV accelerations in our study are LATV suspension systems and the LATV operator driving.

The peak ATD resultant chest and head accelerations measured during LATV emergency driving maneuvers in this study were generally higher than those measured during LATV normal driving. The range of peak ATD resultant chest acceleration was $0.31-3.15 \mathrm{~g}$ during emergency driving and $0.25-0.38 \mathrm{~g}$ under normal driving conditions. The range of peak ATD resultant head acceleration was 0.33-4.65g during emergency driving and $0.33-0.44 \mathrm{~g}$ under normal driving conditions. It should be noted that peak ADT accelerations could not be measured when the ATD impacted the LATV interior. These impacts are associated with accelerations beyond the range of the accelerometer 
sensors used in this study (impact instances occurred in WTORS configurations A and B, indicated with EX in Tables 4 and 5). Sprigle and Linden ${ }^{24}$ also reported mean wheelchair passenger trunk accelerations under normal driving conditions in a modified van: acceleration $(0.028-0.151 \mathrm{~g})$, braking $(0.086-0.363 \mathrm{~g})$, and turning maneuvers $(0.062-$ $0.194 \mathrm{~g})$. The ATD chest accelerations in our study of LATV emergency maneuvers were higher than those found by Sprigle and Linden ${ }^{24}$ in van normal driving (perhaps because vehicle accelerations in our study were also higher).

Under proper WTORS use (configuration C), WTORS loading was generally higher during emergency driving measured in this study than during normal. Highest peak front tiedown loading (FRTD $135.5 \mathrm{lb}$, FLTD $53.0 \mathrm{lb}$ ) during emergency driving was higher than during normal driving (FRTD $19.0 \mathrm{lb}$, FLTD $16.4 \mathrm{lb}$ ). Highest peak rear tiedown loading (RRTD $138.4 \mathrm{lb}$, RLTD $44.9 \mathrm{lb}$ ) during emergency driving was also higher than during normal driving (RRTD $14.8 \mathrm{lb}$, RLTD $14.3 \mathrm{lb}$ ). While LB and SB loads were negligible during normal driving, highest peak LB load was $34.0 \mathrm{lb}$ and the highest peak SB load was 107.2 lb. Even though WTORS loads during emergency driving were higher than those during normal driving, they were substantially less than those measured in high-g frontal impact studies ${ }^{30,32,33}$ (22.5-471.0 lb per front tiedown, 1400.1-4728.4 lb per rear tiedown) and high-g rear impact studies ${ }^{31,34}$ (1079.1-1765.0 lb per front tiedown, 57.8-427.1 lb per rear tiedown).

The findings in this study suggest that when WTORS are properly used, wheelchair passengers are more likely to safely endure an LATV emergency driving maneuver. When WTORS are properly used, the ATD remains seated in the wheelchair, while the wheelchair remains relatively stationary within the securement station. The 
only wheelchair contact with securement station boundaries is with the folded-seat (nonpadded, de facto barrier) under the window. The securement station set-up in this study was such that the wheelchair's right wheel was approximately 0.5 in from folded-seat barrier. Thus, lateral wheelchair frame flexing can cause wheelchair contact with the folded-seat barrier. This minor contact is benign and can be considered negligible as it does not increase wheelchair passenger injury risk.

The securement station right side folded-seat barrier prevented wheelchair and ATD impact with the LATV window. This is an important observation to consider as researchers attempt to design alternative methods of wheelchair securement through means of "containment". However, more research is needed to determine if containment devices are safe for wheelchair passengers on LATVs.

With WTORS disuse or misuse, wheelchair passengers are at greater risk of injury. This is evidenced by the high frequency of ATD ejections from the wheelchair (57\% WTORS disuse, 64\% WTORS misuse) and the wheelchair tipping over (36\% WTORS misuse). ATD ejection frequency was even higher when examining any maneuver that involved a form of LATV braking (braking, left-turn/braking, and rightturn/braking) or turning right. With WTORS disuse and misuse, the ATD was ejected from the wheelchair in all maneuvers that involved a form of LATV braking or turning right. Under these conditions, the ATD continues to move forward or to the left and into the aisle. If there is no LB or SB to keep the ATD in the wheelchair, the ATD's forward or lateral momentum causes ejection. The only times the ATD was offered limited ejection protection is during LATV accelerations and left turns. The ATD was not ejected during any acceleration maneuver and remained seated in the wheelchair during two out 
of three left turn maneuvers. During LATV acceleration, the ATD's inertia tends to cause rearward ATD motion, so the ATD is typically contained within the wheelchair by the wheelchair seatback. As previously mentioned, the folded-seat may serve as a barrier to prevent wheelchair and ATD excursions to the right that would typically occur during a left turn.

There are several limitations associated with this study. An ATD was used to represent the wheelchair passenger, which may not be an ideal representation. The ATD was designed based on the $50^{\text {th }}$ percentile anthropometric measures of the general male population; these measures may not be the same for the wheelchair passenger population. Additionally, the ATD was developed and validated for use in a high-g (20g) crash environment, while our study was conducted in a low-g $(<1.26 \mathrm{~g})$ setting. The ATD's joint properties may be too stiff to accurately simulate actual wheelchair passenger response in a low-g environment. However, since surrogate humans based on the wheelchair population and/or surrogate humans validated for low-g environments have not yet been developed, the ATD used in this study is a reasonable first step in representing a wheelchair passenger.

The wheelchair used in our study was an ultra-light, $\mathrm{x}$-braced frame, manual wheelchair; other wheelchairs may have produced different tiedown loading or different wheelchair accelerations. Also, our study was limited to only one LATV make and model, and one LATV operator; varying LATV models and LATV operators may have produced different LATV accelerations resulting in different wheelchair and ATD accelerations and tiedown loading. 
Some data was not measurable during testing because of limited accelerometer range $(0 \pm 6 \mathrm{~g})$. This occurred specifically when the wheelchair and ATD impacted the LATV interior. Our study did not provide ATD pelvic accelerations because of a malfunctioning accelerometer. Additionally, the number of trials for each emergency maneuver was limited under WTORS disuse and misuse configurations (A and B) because of the threat of equipment damage from ATD ejections. Restrictions on LATV and LATV operator time did not allow us to repeat testing.

In conclusion, we found that LATV accelerations, ATD chest and head accelerations, and WTORS loads are higher during emergency driving maneuvers than normal driving maneuvers. When WTORS are properly used, the wheelchair passenger does not appear to be at increased risk of injury, while WTORS disuse and misuse are associated with a high frequency of wheelchair passenger ejection from the wheelchair, especially during an emergency maneuver that involves any form of LATV braking (braking, left-turn/braking, and right-turn/braking). 
CHAPTER 5

\author{
MANUAL WHEELCHAIR EXCURSIONS UNDER VARYING WHEELCHAIR \\ BRAKE MOUNTING HARDWARE CONDITIONS DURING LARGE ACCESSIBLE \\ TRANSIT VEHICLE NORMAL DRIVING
}

\begin{abstract}
Overview
Large accessible transit vehicles (LATVs) are generally considered a safe mode of transportation, but have been proven less safe for persons who are seated in their wheelchairs during transit. There are documented disparities between transit provider practices regarding the use of four-point, strap-type wheelchair tiedown and occupant restraint systems (WTORS) to secure wheelchairs and restrain wheelchair-seated passengers during LATV transit. Additionally, recent research suggests WTORS disuse and misuse is prevalent onboard LATVs, which has, in part, led to the design and development of alternative approaches to wheelchair securement and containment. Some of these alternatives may rely, to some extent, on wheelchair brakes to limit wheelchair excursions. The objective of this portion of the study was to report wheelchair excursions in LATV normal driving for two manual wheelchair brake mounting hardware conditions: securely clamped to the wheelchair frame vs. loosely clamped to the wheelchair frame.
\end{abstract}


The wheelchair passenger was represented by an anthropomorphic test device (ATD) seated in a transit, manual, X-braced-frame wheelchair. The wheelchair securement station was located on the right side of the LATV. The ATD was not restrained while the wheelchair was secured only with the lap belt routed around the wheelchair seatback (common WTORS misuse). A total of eight 2-minute normal driving trials were performed (four trials with securely clamped brake hardware, four trials with brake mounting hardware where the clamping mechanism had loosened from the wheelchair frame). The results revealed substantially higher wheelchair excursions when the wheelchair brake hardware had loosened from the frame (mean 87.1 +/- 25.1 in per trial) in comparison to when it was securely fastened to the frame (mean $4.0+/-5.0$ in per trial). We also found that during LATV normal driving an improperly secured manual wheelchair can tip over in the aft direction, regardless of brake hardware condition.

\section{Introduction}

Large accessible transit vehicles (LATVs) are considered a safe mode of transportation with a low fatality incidence of the general ridership passengers ${ }^{1}$. This stems in part from large vehicle mass and slow speed of travel in city traffic. Despite this relatively safe environment, LATVs may not be as safe for wheelchair seated passenger ${ }^{4}$, ${ }^{6}$, even under normal driving conditions ${ }^{7}$.

Wheelchair users depend on public transit to access employment, education, health care, and recreation. It is necessary to afford wheelchair passengers the same level of transportation safety as the general population. The Americans with Disabilities Act (ADA) ${ }^{13}$ requires the provision of a wheelchair securement device and occupant 
restraints (lap and shoulder belts), but does not mandate their $u^{8} e^{8}$. As a result, there are disparities across transit agency policies with respect to WTORS use ${ }^{10}$. Disuse and misuse of WTORS have been suspected to place wheelchair-seated passengers at greater risk of injury ${ }^{3}$. In our previous study ${ }^{21}$, we reviewed video surveillance records of WTORS usage on LATVs and found that the majority (76\%) of wheelchairs were not secured using four point strap-type tiedowns during transport, and that misuse of the lap belt was common ( $44 \%$ of cases). Lap belt misuse consisted of the LATV operators routing the lap belt around the wheelchair seatback in an attempt to secure the wheelchair. The lap belt was used to restrain the wheelchair passenger in only $20 \%$ of observed cases.

Alternative solutions to wheelchair securement or retention such as auto-docking ${ }^{18}$ and forward-facing ${ }^{22}$ and rear-facing ${ }^{17}$ wheelchair passenger stations are being explored, in part, because of reported disuse and misuse of strap-type, four-point tiedowns and occupant restraint systems (ORS) in LATVs. As these alternative systems are being evaluated for feasibility and safety with respect to wheelchair securement and wheelchair passenger restraint in LATV transit, failure of wheelchair components to function properly can adversely affect wheelchair stability in LATV travel. Wheelchair transportation research has already yielded the design and introduction of transit-safe wheelchairs (conforming to the ISO 7176-19 standard "Wheeled mobility devices for use as seats in motor vehicles" ${ }^{28}$ and ANSI/RESNA WC19 standard "Wheelchairs used as seats in motor vehicles" ${ }^{36}$ ). These transit-safe wheelchairs must meet design criteria and have to prove crashworthy in high-g $(20 \mathrm{~g})$ frontal-impact. It is reasonable to assume that wheelchairs that are able to withstand $20 \mathrm{~g}$ frontal impact perform well in a low-g $(<3 \mathrm{~g})$ 
environment, provided that WTORS are used properly. However, in WTORS disuse and misuse circumstances, the wheelchair must rely on its brake system to prevent wheelchair excursion. Given the prevalence of WTORS disuse and misuse on LATVs, we evaluated the influence of manual wheelchair brake effectiveness under LATV normal driving conditions.

\section{Methods}

This portion of the study focused on describing wheelchair excursions with a common misused WTORS configuration (lap belt routed around the wheelchair seatback in an attempt to secure the wheelchair) during normal LATV driving. Under these conditions, we investigated two different manual wheelchair brake hardware conditions: securely clamped to the wheelchair frame vs. loosely clamped to the wheelchair frame.

The LATV (Gillig, Year: 2010, Model \# G30D102N4) used in the experimental evaluation was a hybrid, $40 \mathrm{ft}(12.2 \mathrm{~m})$, low floor, pneumatic suspension, fixed-route, intra-city bus. The route used for testing connects various medical facilities in a mediumsized metropolitan city; a relatively level-terrained $(461 \pm 9 \mathrm{ft}, 141 \pm 3 \mathrm{~m}$ above sea level), urban setting with stop-and-go, inner-city traffic. This route was selected because of a high prevalence of wheelchair passengers. The testing included a total of 16 acceleration, 12 braking, 2 left turn, and 1 right turn maneuvers. Testing was performed in dry weather conditions and excluding the research group, no passengers were on board the LATV during testing. The same licensed LATV operator was used during all testing and was instructed to drive as if the LATV were in service (making all stops, obeying standard traffic laws, kneeling the LATV if necessary, etc.) 
The wheelchair passenger was represented by a wheelchair-seated, $50^{\text {th }}$ percentile Hybrid II anthropomorphic testing device (ATD - $74.5 \mathrm{~kg}$,) with abdominal insert removed to better simulate wheelchair passenger kinematics in LATV transit ${ }^{37}$. The wheelchair (Quickie 2, Sunrise Medical) used during testing was an ultra-light, X-braced, manual wheelchair with the transit option (conforming to the ISO 7176-19 standard "Wheeled mobility devices for use as seats in motor vehicles" ${ }^{28}$ ). The wheelchair was adjusted to properly fit the ATD and featured a medium contoured seatback (J3, Jay Seating Systems) and a 2.8 in contoured seat cushion (Ride Designs). Figure 1 illustrates the test set up.
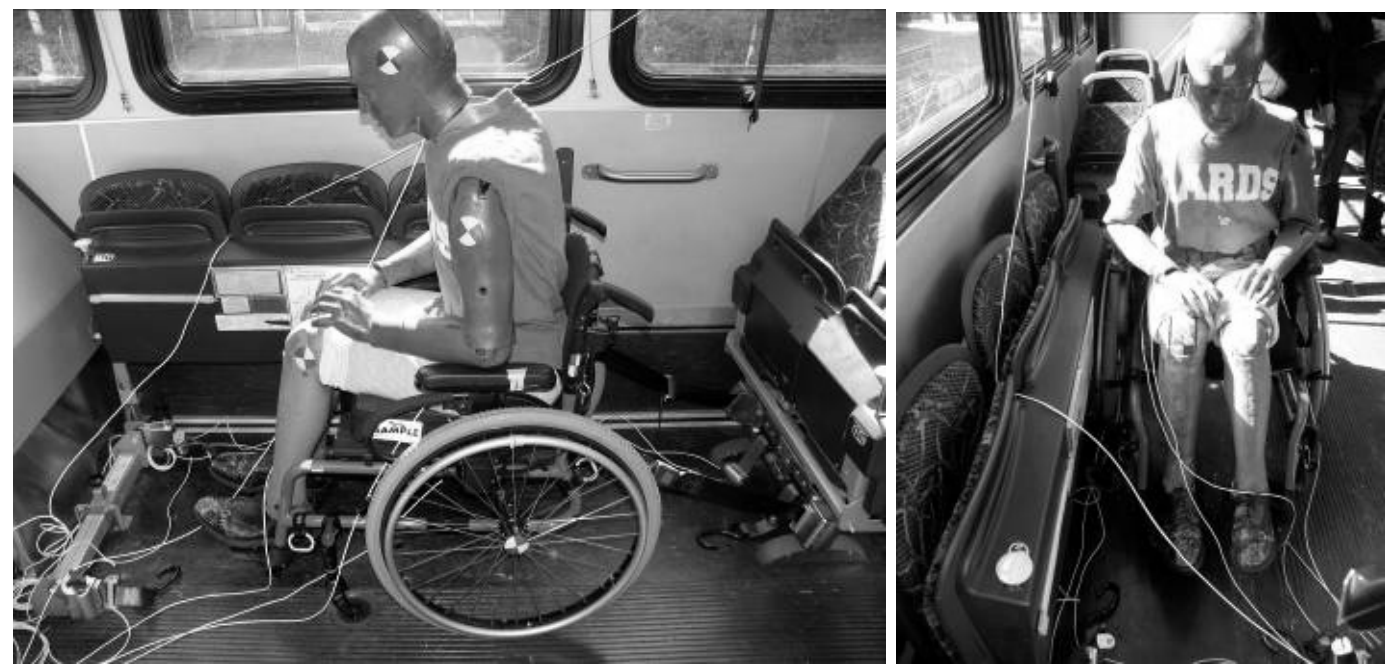

Figure 1A. Test setup of $50^{\text {th }}$ percentile ATD seated in manual wheelchair within LATV securement station. The lap belt was used in an attempt to secure the wheelchair (routed around the seatback). The tiedowns and shoulder belt were not applied. 


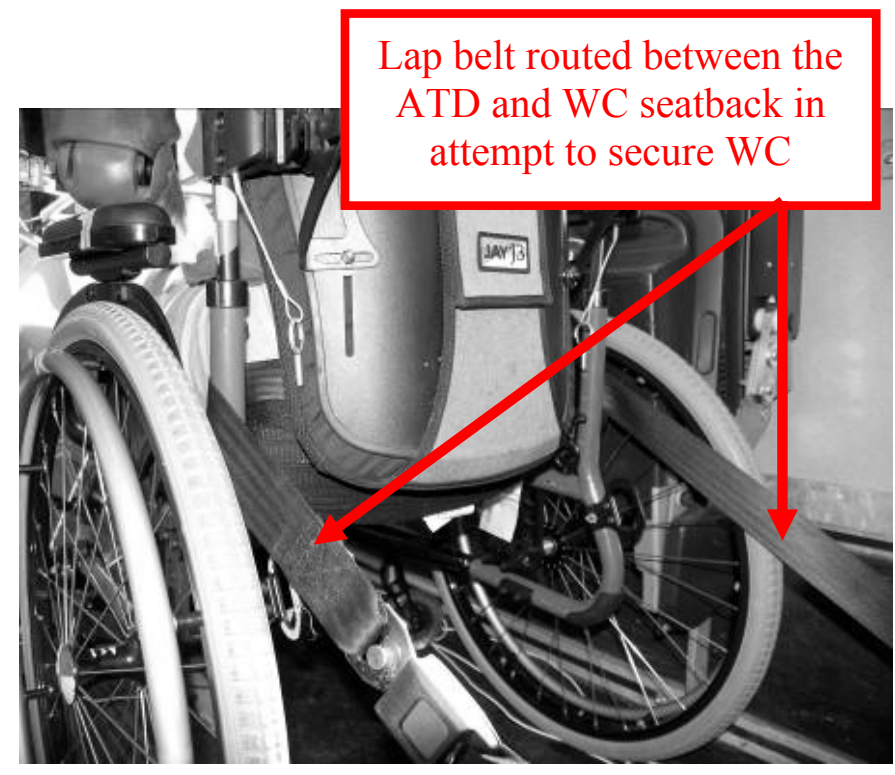

Figure 1B. Test setup of $50^{\text {th }}$ percentile ATD seated in manual wheelchair with lap belt used in an attempt to secure the wheelchair (routed around the seatback). The tiedowns and shoulder belt were not applied.

The wheelchair was secured at the securement station on the right side of the LATV (previous experience showed that wheelchair passengers use the right securement station most often). The securement station within the LATV conformed to ADA regulation with respect to size (measured size: 66 in long by 36 in wide). The four-point, strap-type, tiedowns were not used to secure the wheelchair; instead, the retractor-style lap belt (SURE-LOK, AL701060M-AMS) was used to in an attempt to secure the wheelchair by positioning the lap belt between the ATD and wheelchair seatback. Previous observation showed (published in our previous study ${ }^{38}$ ) using the lap belt in an attempt to secure the wheelchair was the most common WTORS configuration in LATV transit.

To investigate the effect of the manual wheelchair brake mounting hardware condition on wheelchair stability in normal LATV driving, we compared wheelchair 
excursions associated with two brake hardware mounting conditions: securely clamped to the wheelchair frame vs. loosely clamped to the wheelchair frame. Figure 2 shows the wheelchair brake mounting hardware design and how the mounting hardware was adjusted. To securely clamp the brake to the wheelchair frame, two bolts were tightened to lock the position rod inside the mounting bracket. Once the bolts are tightened, the position rod cannot move relative to the mounting bracket and wheelchair frame. With a fixed position rod, the impingement lever, attached to the position rod, is able to effectively impinge the rear wheel and prevent rear wheel rotation with respect to the wheelchair frame when the brake is engaged using the activation lever. The loosely clamped to the wheelchair frame condition was achieved by first securely clamping the brake to the wheelchair frame bilaterally, as described above, and subjecting the wheelchair with the seated ATD (Figure 1) to approximately 80 min of LATV normal driving (described in more detail in chapter 2). During this time, the vibrations (from LATV engine and transmission, road surface conditions, etc.) loosened the bolts that fix the position rod to the mounting bracket in both wheelchair brakes. This loosened brake mounting hardware condition would be typically associated with prolonged LATV transit. For both wheelchair brake hardware mounting conditions, the brakes were engaged bilaterally by pulling the activation lever toward the rear wheel, forcing the impingement lever to press against the rear wheel tire. 


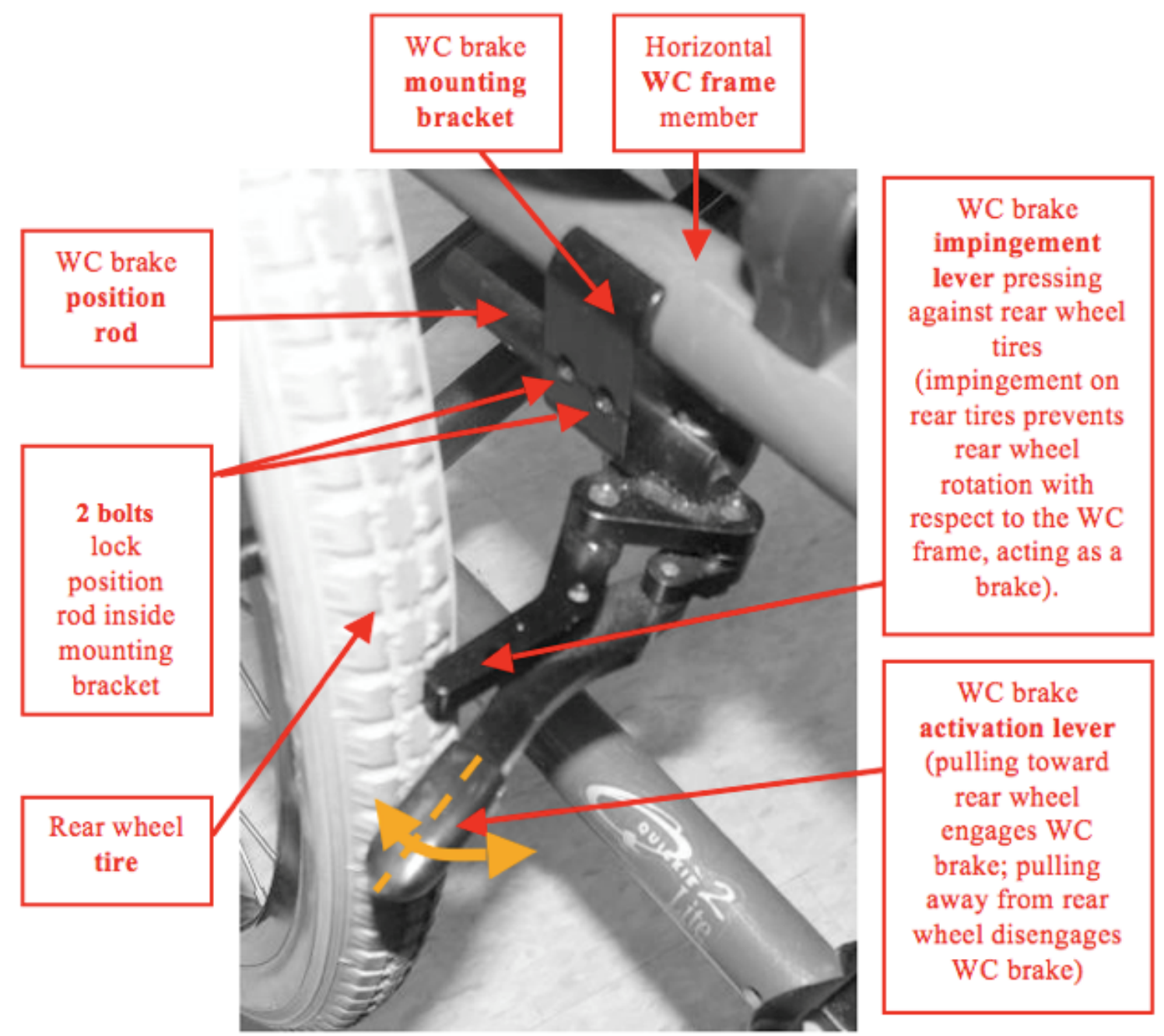

Figure 2. Design of manual wheelchair (compact scissor) brake. The brake is shown in the engaged position. To securely clamp the brake to the wheelchair frame, the two bolts were tightened to lock the position rod inside the mounting bracket. With the position rod fixed relative to the mounting bracket (and therefore the wheelchair frame), the impingement lever (attached to the position rod) was able to effectively impinge the rear wheel tire to prevent rear wheel rotation with respect to the wheelchair frame.

We performed four LATV normal driving trials with the wheelchair and wheelchair seated ATD for each brake mounting hardware condition. One trial consisted of 2 minutes of LATV normal driving along the previously described route. The brake mounting hardware was loosely clamped to the wheelchair frame for the first four trials, while trials five through eight featured securely clamped brake mounting hardware. At the conclusion of each trial the wheelchair and ATD were reset to their original position. 
To track wheelchair excursions for each trial, high contrast markers were placed on the wheelchair rear wheel center hub (rear-axle) and wheelchair frame (front left vertical frame member). These markers and front-view and side-view video data (30 frames per second) were used to track wheelchair fore-aft and lateral excursions with MaxTRAQ v. 2.3.2.3 video analysis software.

We summed the measured wheelchair excursions in each trial to calculate the total wheelchair excursion for that trial. The total wheelchair excursion means were compared across the two brake mounting hardware conditions.

\section{Results}

While documenting wheelchair excursions, we observed discrete events for each trial. These events were end points that led to a change in wheelchair excursion direction. A list of events is included below the wheelchair trajectory map for each trial (Figures 3$10)$.

Figure 3 shows wheelchair trajectory during trial 1 . In trial 1, the wheelchair rolled to strike both the front securement station barrier (front bulkhead) and rear securement station barrier (rear bulkhead). The wheelchair also tilted rearward and rotated laterally into the aisle. 

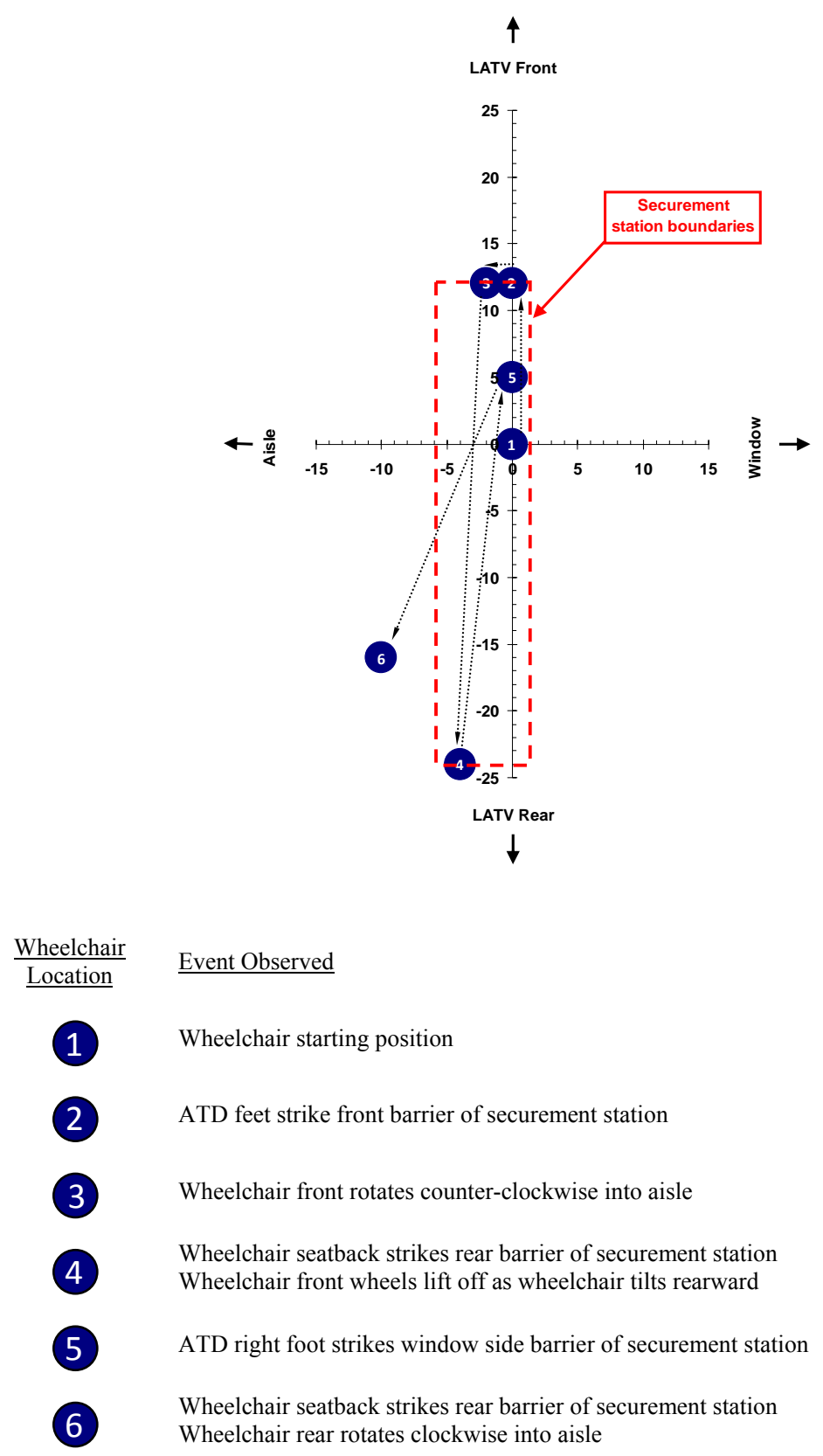

Figure 3. Wheelchair trajectory during trial 1 (brake mounting hardware loosely clamped to the wheelchair frame). The dark-shaded circular markers represent wheelchair locations (rear axle) at sequential points over trial duration. Dimensions are in inches.

Figure 4 shows wheelchair trajectory during trial 2. In trial 2 the wheelchair rolled to strike the rear securement station barrier, tilted rearward, and rotated laterally into the aisle. 


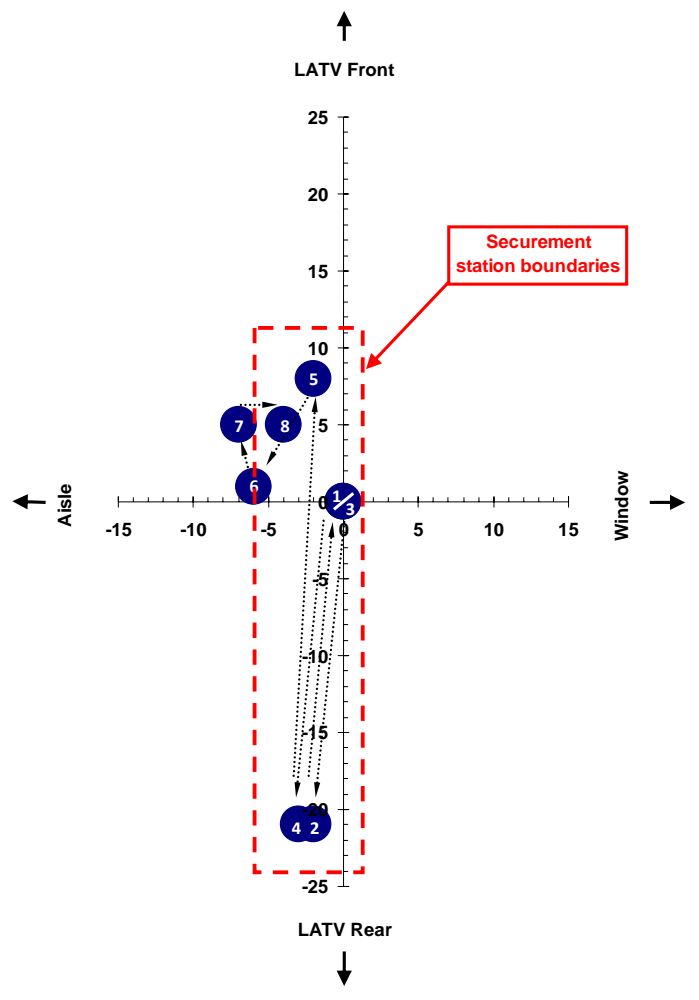

Wheelchair Event Observed
(1) Wheelchair starting position
Wheelchair seatback strikes rear barrier of securement station
Wheelchair front wheels lift off as wheelchair tilts rearward
(4) Wheelchair rolls back to initial position
(5) Wheelchair seatback strikes rear barrier of securement station
(6) Wheelchair rolls forward
(7) Wheelchair front rotates counter-clockwise into aisle
(8)

Figure 4. Wheelchair trajectory during trial 2 (brake mounting hardware loosely clamped to the wheelchair frame). The dark-shaded circular markers represent wheelchair locations (rear axle) at sequential points over trial duration. Dimensions are in inches. 
Figure 5 shows wheelchair trajectory during trial 3. During trial 3 the wheelchair rolled to strike the rear securement station barrier, tilted rearward, and also rotated laterally into the aisle. 

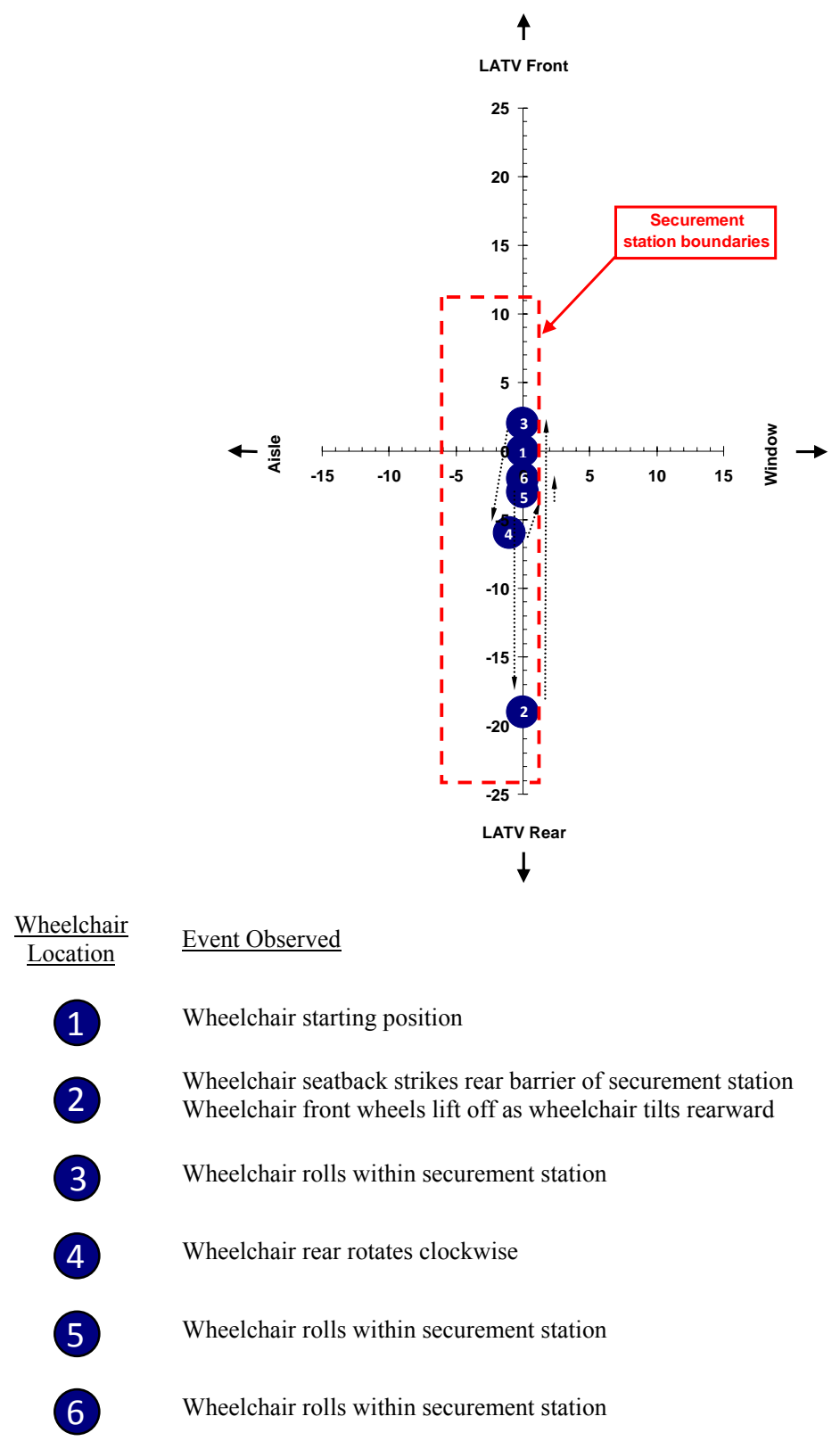

Figure 5. Wheelchair trajectory during trial 3 (brake mounting hardware loosely clamped to the wheelchair frame). The dark-shaded circular markers represent wheelchair locations (rear axle) at sequential points over trial duration. Dimensions are in inches.

Figure 6 shows wheelchair trajectory during trial 4. In trial 4 the wheelchair rolled to strike both the front and rear securement station barriers, tilted rearward, as well as rotated laterally into the aisle. During trial 4, the wheelchair ultimately flipped over rearward resulting in ATD head impact with the rear securement station barrier. 

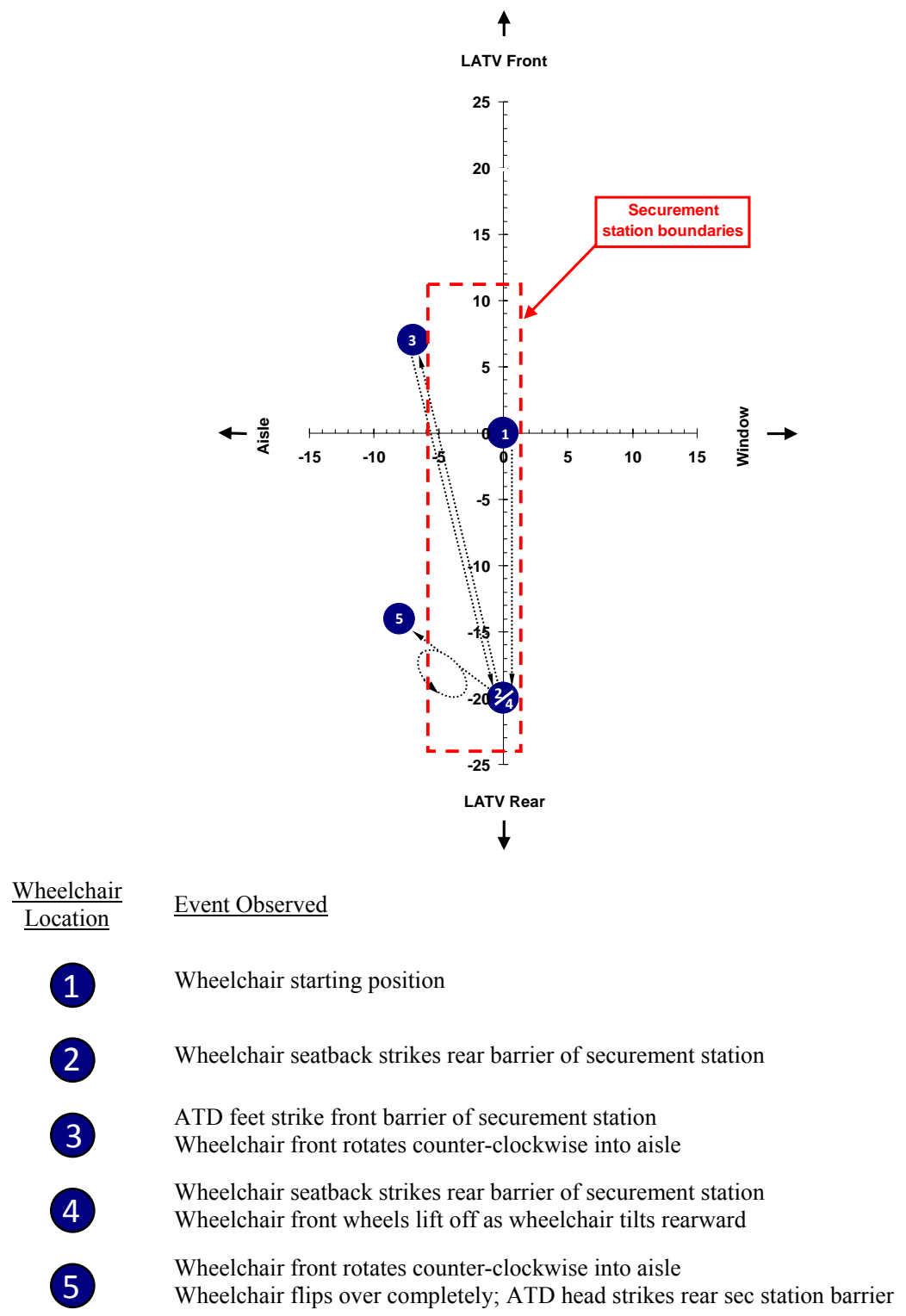

Figure 6. Wheelchair trajectory during trial 4 (brake mounting hardware loosely clamped to the wheelchair frame). The dark-shaded circular markers represent wheelchair locations (rear axle) at sequential points over trial duration. Dimensions are in inches.

Figure 7 shows wheelchair trajectory during trial 5. During trial 5 the wheelchair flipped over rearward resulting in ATD head impact with the rear securement station barrier. 

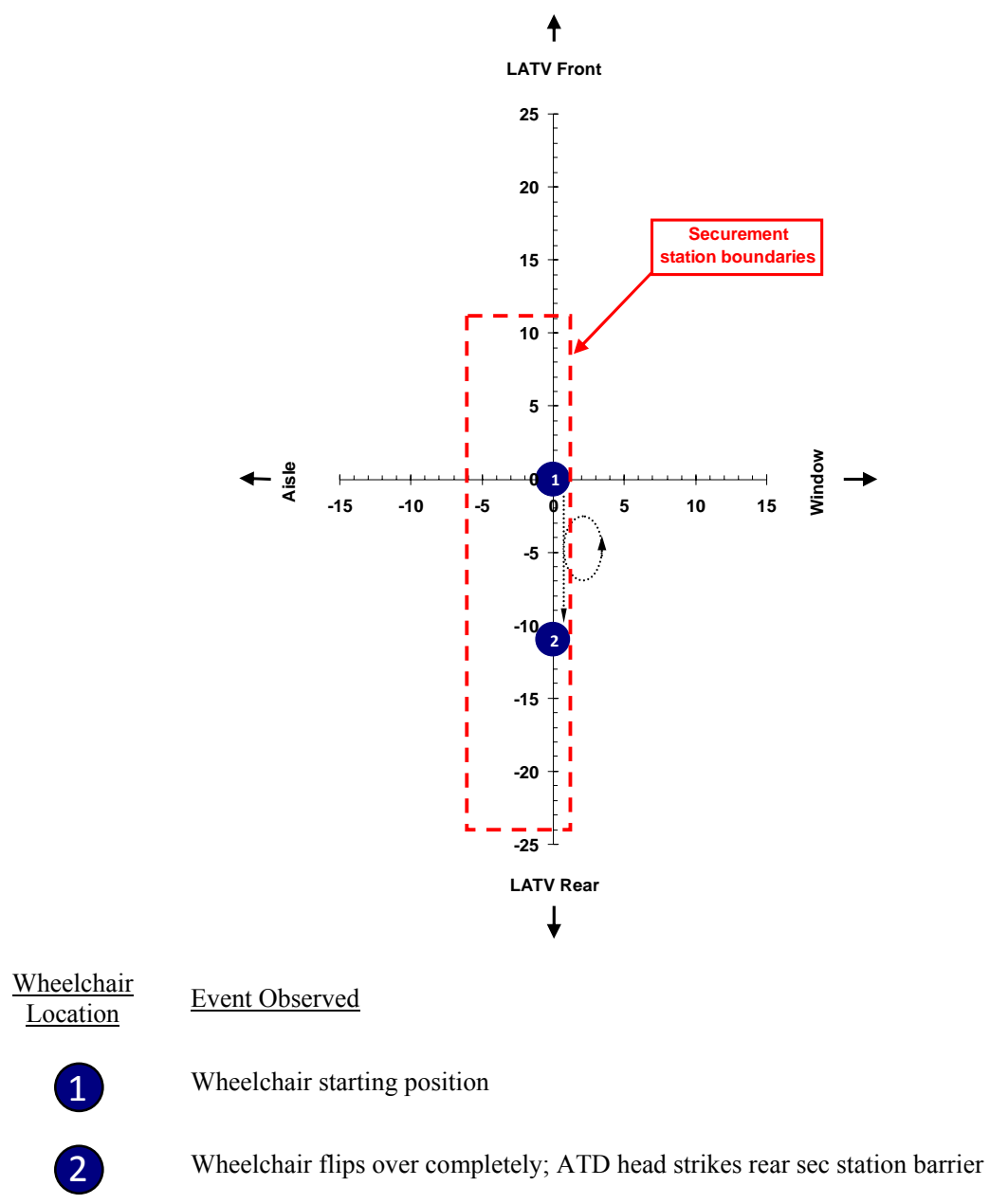

Figure 7. Wheelchair trajectory during trial 5 (brake mounting hardware securely clamped to the wheelchair frame). The dark-shaded circular markers represent wheelchair locations (rear axle) at sequential points over trial duration. Dimensions are in inches.

Figure 8 shows wheelchair trajectory during trial 6 . In trial 6 the wheelchair rolled forward slightly. 

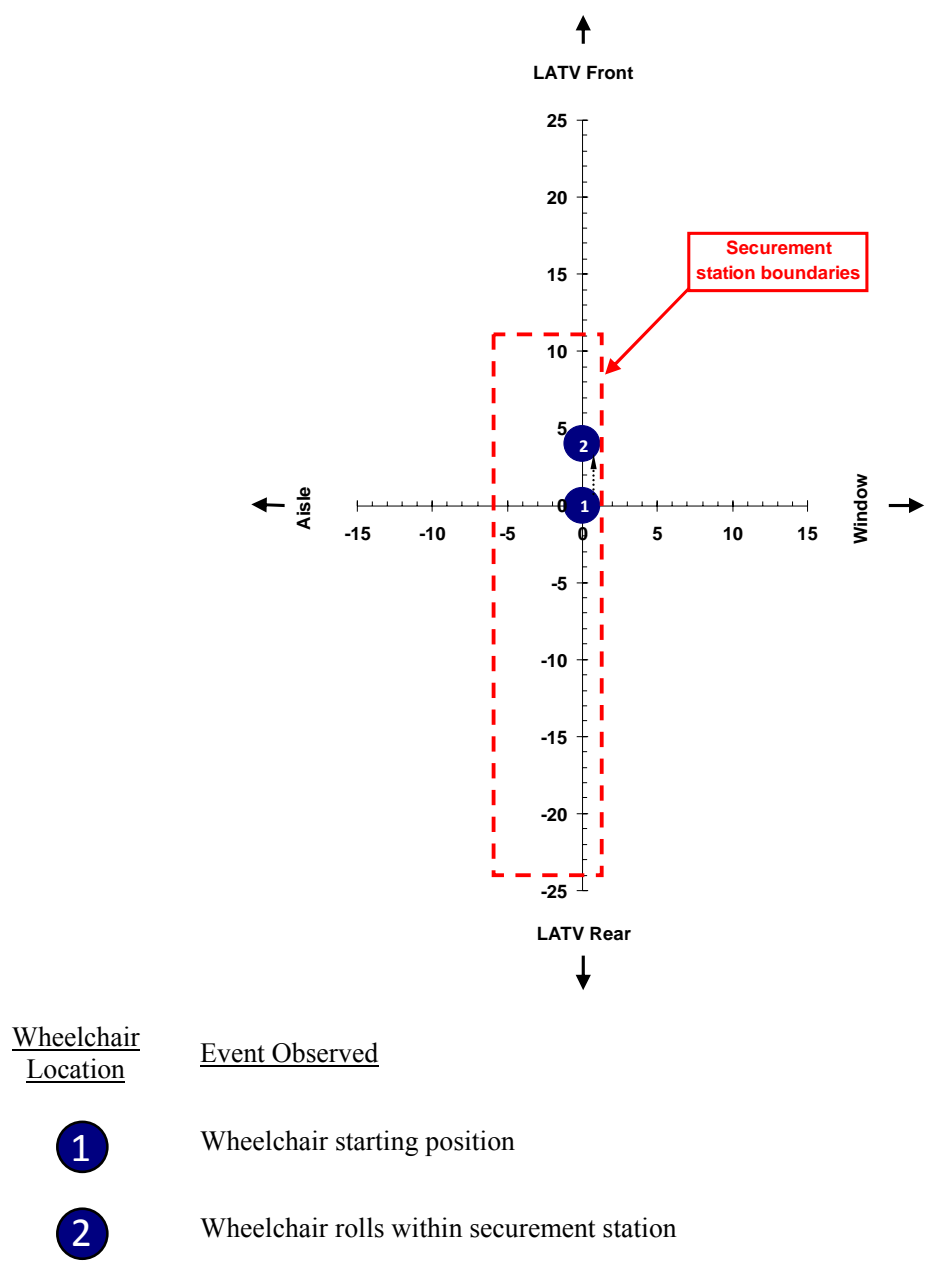

Figure 8. Wheelchair trajectory during trial 6 (brake mounting hardware securely clamped to the wheelchair frame). The dark-shaded circular markers represent wheelchair locations (rear axle) at sequential points over trial duration. Dimensions are in inches.

Figure 9 shows wheelchair trajectory during trial 7. In trial 7 the wheelchair rolled forward slightly. 

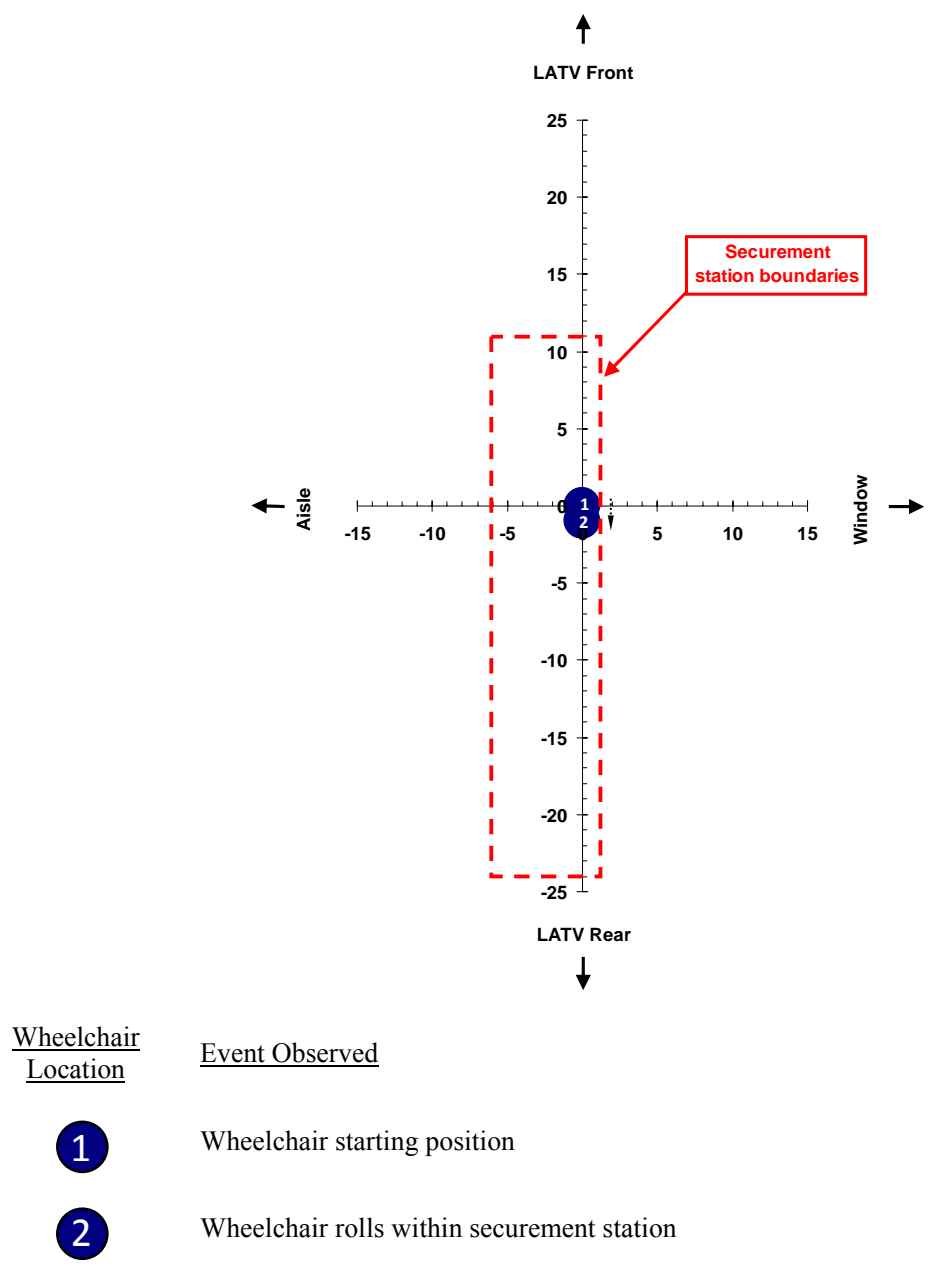

Figure 9. Wheelchair trajectory during trial 7 (brake mounting hardware securely clamped to the wheelchair frame). The dark-shaded circular markers represent wheelchair locations (rear axle) at sequential points over trial duration. Dimensions are in inches.

Figure 10 shows wheelchair trajectory during trial 8 . Throughout trial 8 the wheelchair remained in its original position. 

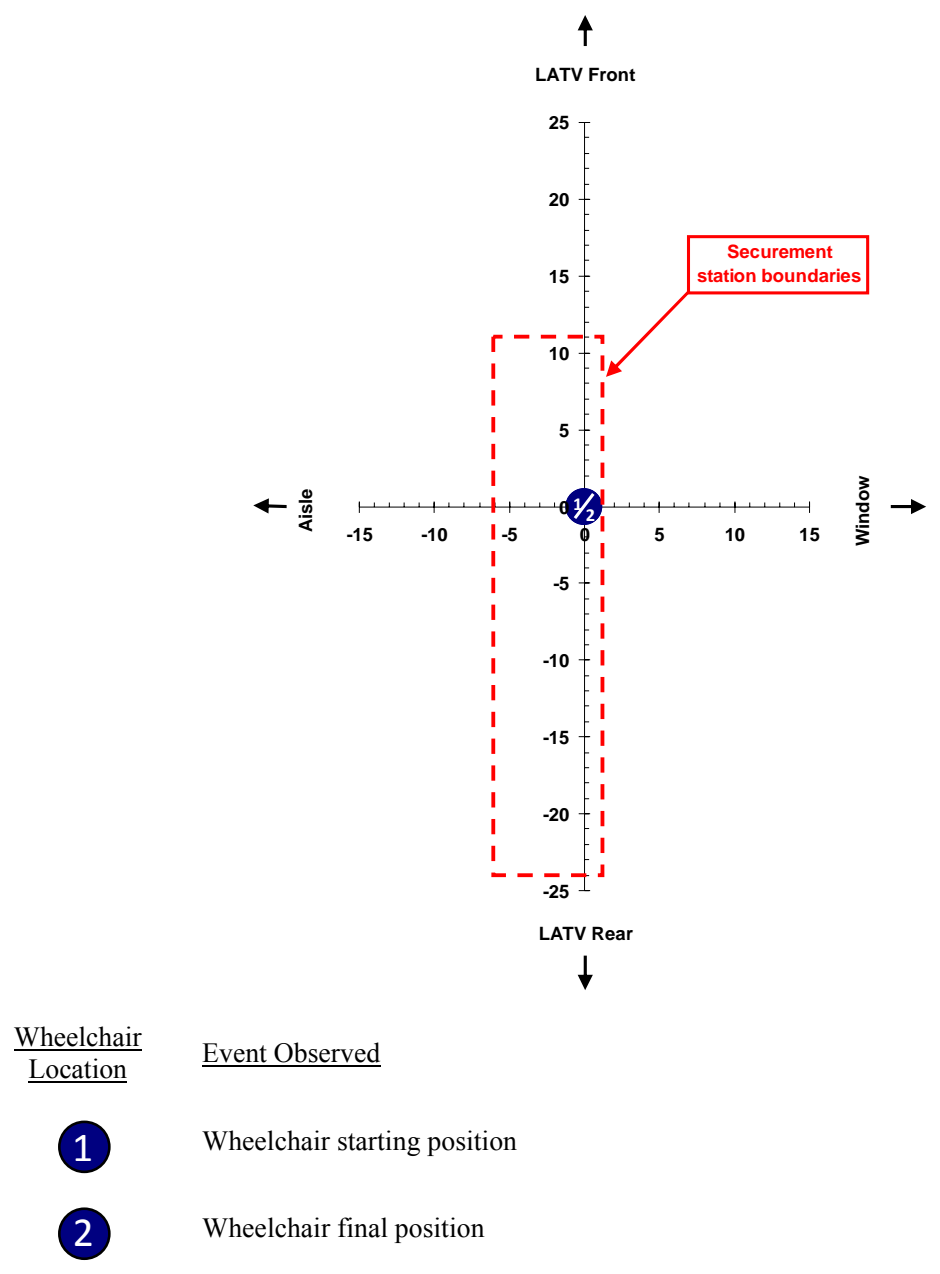

Figure 10. Wheelchair trajectory during trial 8 (brake mounting hardware securely clamped to the wheelchair frame). The dark-shaded circular markers represent wheelchair locations (rear axle) at sequential points over trial duration. Dimensions are in inches.

Table 1 shows the total wheelchair excursions (total distance wheelchair moved) in each trial across both brake mounting hardware conditions. The mean wheelchair excursion when brake mounting hardware was loosely clamped to the wheelchair frame was 87.1 in (+/- $25.1 \mathrm{in})$, while the mean wheelchair excursion was $4.0 \mathrm{in}(+/-5.0 \mathrm{in})$ when brake mounting hardware was securely clamped to the wheelchair frame. 


\begin{tabular}{|c|c|c|c|}
\hline \multicolumn{4}{|c|}{ Total wheelchair excursion (in) } \\
\hline Trial & $\begin{array}{c}\text { WC brake mounting } \\
\text { hardware loosely } \\
\text { clamped to WC frame } \\
\text { WC brake mounting } \\
\text { hardware securely } \\
\text { clamped to WC frame }\end{array}$ & Trial \\
\hline 1 & 102.6 & 11.0 & 5 \\
\hline 2 & 107.7 & 4.0 & 6 \\
\hline 3 & 52.2 & 1.0 & 7 \\
\hline 4 & 85.8 & 0.0 & 8 \\
\hline \hline Mean & $\mathbf{8 7 . 1}$ & $\mathbf{4 . 0}$ & Mean \\
\hline$+/-$ & 25.1 & 5.0 & $+/-$ \\
\hline
\end{tabular}

Table 1. Total wheelchair excursions (distance wheelchair moved) for each trial across both brake mounting hardware conditions.

\section{Discussion and Conclusions}

When the brake mounting hardware was loosely clamped to the wheelchair frame, we observed substantially higher wheelchair excursion (mean $87.1+/-25.1$ in) than when it was securely clamped to the wheelchair frame (mean $4.0+/-5.0 \mathrm{in}$ ). This finding suggests that, even under normal LATV driving, the wheelchair and wheelchair occupant has a high likelihood of contacting securement station boundaries, the LATV interior, and/or other LATV passengers. This may place the wheelchair occupant (and possibly other LATV passengers) at increased risk for injury.

The retractor locking mechanism of the lap belt (misused in an attempt to secure the wheelchair) did not effectively engage during LATV normal driving. This became especially evident when the brake mounting hardware was loosely clamped to the wheelchair frame, as the wheelchair brake did not effectively prevent forward wheelchair excursions. Although the lap belt was misused, it is reasonable to expect that during LATV deceleration (such as a normal braking maneuver) the belt retractor locking 
mechanism would engage and prevent forward wheelchair excursions. However, it should be noted that the lap belt could not have prevented rearward wheelchair excursions during LATV acceleration maneuvers. During LATV acceleration and WTORS misuse, the wheelchair passenger has to rely solely on the manual wheelchair brake to prevent wheelchair excursions. The four-point, strap-type WTORS (such as the one in the LATV used in this study) were designed to keep wheelchairs secured and wheelchair occupants restrained in a high-g crash environment. WTORS retractor locking mechanisms were designed to engage in a high-g crash environment, but they may not engage in a low-g $(<1 \mathrm{~g})$ environment associated with normal LATV driving maneuvers. It is important to point out that initial WTORS designs did not feature retractors with locking mechanisms, but simple belts that were anchored directly to the vehicle. Because of finite belt length, we can assume these belts performed better than belts with retractors (such as those employed in our testing) under LATV normal driving conditions.

However, the fixed-end belts typically remained on the vehicle floor when not in use and can become soiled over time. Their soiled condition may be related to documented WTORS disuse, as wheelchair passengers refuse to have soiled WTORS contacting their person and/or wheelchair ${ }^{10}$.

The wheelchair tipped over in the first trial when the brake mounting hardware was securely clamped to the wheelchair frame (trial 5). This suggests that while securely clamped brake mounting hardware may be effective for preventing wheelchair excursions in general, eliminating rolling of the rear wheels does not eliminate all risks. A reasonable assumption is that proper WTORS use would have been prevented the tipover event. For the test setup in this study, the two factors that worked in conjunction to 
lead to the wheelchair and wheelchair passenger tip-over during an LATV acceleration maneuver in trial 5 are (1) a relatively short wheelchair wheelbase (distance between front casters and rear wheels) and (2) the ATD's inability to balance. Typically, the wheelbase is minimized for manual wheelchair users during wheelchair fitting, as to make wheelie and turning maneuvers easier to perform. Both fore/aft tilt and turning radius of a manual wheelchair are important for general wheelchair maneuverability but are usually "tuned" to the wheelchair user's trunk balancing ability. While the wheelchair used in this study was properly fitted to the wheelchair passenger, represented by an ATD, the ATD did not possess balancing ability. It is possible that a wheelchair user with some ability to balance may not have tipped-over during this LATV acceleration maneuver in trial 5. Nonetheless, this finding underscores the importance of professional, licensed wheelchair suppliers and the need to educate them about potential hazards manual wheelchair passengers face in LATV transit.

Clinical experience suggests that wheelchair users often need manual wheelchair brake adjustments when visiting with their assistive technology clinician or wheelchair supplier. Adjustment typically involves securely clamping brake mounting hardware to the wheelchair frame. These relatively frequent adjustment requests on the part of the wheelchair users suggest that manual wheelchair brake mounting hardware loosens during routine, daily wheelchair use even outside of the LATV environment. It is sensible to think that manual wheelchair brake mounting hardware integrity may be compromised further when the brake is subjected to LATV vibrations present during transit. We therefore suggest transit wheelchair manufacturers focus on improved manual wheelchair brake design. To date, we are not aware of any other study that has investigated the effect 
of the manual wheelchair brake mounting hardware condition on wheelchair stability in normal LATV driving.

The need for continuous manual wheelchair brake adjustment has led to one alternative manual wheelchair brake design that does not rely on any brake components impinging the rear wheel tires, but prevents rear wheel rotation by means of a slotted disk (mounted on the axle) receiving a lock-pin when the brake is engaged. This is a fairly new option for manual wheelchairs and is considered a "luxury" feature that is typically not covered by medical insurance. However, this alternative manual wheelchair brake design would not have likely prevented the tip-over we observed in trial 5.

There are several limitations associated with our study. An ATD was used to represent the wheelchair passenger, which may not be an ideal representation. The ATD was designed based on the $50^{\text {th }}$ percentile anthropometric measures of the general male population; these measures may not be the same for the wheelchair passenger population. Additionally, the ATD was developed and validated for use in a high-g crash environment, while our study was conducted in a low-g setting. The ATD's joint properties may be too stiff to accurately simulate actual wheelchair passenger response in a low-g environment. However, as surrogate humans based on the wheelchair population and/or surrogate humans validated for low-g environments have not yet been developed, the ATD used in this study is a reasonable first step in representing a wheelchair passenger. The wheelchair used in our study was an ultra-light, x-braced frame, manual wheelchair; other wheelchairs may have produced different results. The manual wheelchair brakes used in our study were compact scissor wheel locks. Although this brake and associated brake mounting hardware design is common on manual 
wheelchairs, other brake designs may have produced different results. We did not quantify how secure the brake mounting hardware was clamped to the wheelchair frame, and we did not re-secure the brake mounting hardware to the wheelchair frame after each trial for trials $5-8$, so the brakes' robustness may have been diminished in later trials. Additionally, the wheelchair tipping in trial 5 could have influenced robustness of the brake in subsequent trials. The inflation level of the rear tires and rear tire tread could have affected the manual wheelchair brakes' effectiveness. Also, our study was limited to only one LATV make and model, one LATV operator, and one LATV route; varying LATV models, LATV operators, and LATV routes with differing topography may have produced different LATV accelerations resulting in possibly different wheelchair excursions. Finally, our findings only described normal LATV driving maneuvers. Emergency driving maneuvers will likely be associated with increased LATV accelerations, producing possibly higher wheelchair excursions.

As a result of this study, we conclude that manual wheelchair brake mounting hardware can play a role in limiting wheelchair excursions in LATV normal driving. Regular wheelchair maintenance that includes securely clamping the manual wheelchair brake mounting hardware to the wheelchair frame should be performed on a routine and frequent basis, as it can increase wheelchair passenger safety during LATV transit. Nonetheless, WTORS should always be used as intended by the manufacturer to provide the highest level of wheelchair transportation safety in any vehicle, including LATVs. 
CHAPTER 6

DEVELOPMENT AND VALIDATION OF COMPUTER SIMULATION MODEL OF AN ADULT MANUAL TRANSIT WHEELCHAIR WITH A SEATED OCCUPANT IN A LARGE ACCESSIBLE TRANSIT VEHICLE UNDER EMERGENCY BRAKING CONDITIONS

\section{Overview}

Large accessible transit vehicles (LATVs) are generally considered a safe mode of transportation. However, it has been shown that LATVs may not be as safe for persons who are seated in their wheelchairs during transit. Thus far only limited experimental and computer simulation data have been available to study wheelchair transportation safety in large accessible transit vehicle (LATV) transit. Computer modeling can be used as an economic and comprehensive tool to gain critical knowledge regarding wheelchair and wheelchair tiedown and occupant restraint systems (WTORS) loading. This study describes the development and validation of a computer model simulating an adult wheelchair-seated occupant subjected to a LATV emergency braking maneuver. The model was developed in MADYMO ${ }^{\mathrm{TM}}$ and validated rigorously using the experimental results of a combination braking/left-turn maneuver. Outcomes from the model can 
provide critical wheelchair and WTORS loading information to wheelchair and WTORS manufacturers, resulting in safer wheelchair and WTORS designs for use on LATVs.

\section{Introduction}

Large accessible transit vehicles (LATVs) are generally considered a safe mode of transportation with a low fatality incidence of all passengers ${ }^{1}$. This is due in part to overall vehicle mass and the slow speed of LATV travel associated with intra-city routes. Despite this relatively safe environment, studies show that LATVs may not be as safe for passengers who remain in the wheelchairs during transit ${ }^{3,4,6,10}$. While general passenger injuries occur relatively rarely in LATVs, normal and emergency driving maneuvers can cause wheelchair passenger injuries ${ }^{3,7}$. Certainly the proper usage of wheelchair tiedown and occupant restraint systems (WTORS) would be expected to provide safe transit under normal LATV driving conditions. However, WTORS disuse and misuse has been documented $^{3,21}$, in part because the 4-pont, belt type wheelchair tiedowns and occupant restraints are cumbersome to use and do not allow for wheelchair passenger independence with regard to wheelchair securement and passenger restraint. Current research is underway to identify alternative solutions to wheelchair securement in a low-g environment $(<1 \mathrm{~g})$. These alternative solutions include forward-facing wheelchair passenger stations ${ }^{22}$ and auto-docking systems ${ }^{18}$, both of which offer improved ease of use and may exhibit reduced frequencies of disuse and misuse in comparison to the straptype, 4-point wheelchair tiedown and occupant restraint systems. One study ${ }^{17}$ found that wheelchair passengers prefer auto-docking systems compared to 4-pont, belt type wheelchair tiedown and occupant restraint systems in LATV transit. Alternatives to 
traditional strap-type, 4-point WTORS also offer a more independent approach for wheelchair securement or retention, but additional research is needed to demonstrate their feasibility and provision of safety. Experimental testing on LATVs is one method to perform these investigations, but has certain drawbacks. Experimental limitations include their time consuming nature, relatively high costs, and the inability to provide detailed wheelchair structure loading data. Computer simulation modeling addresses some of these limitations and can be an effective tool to supplement experimental testing.

Computer simulation has been used previously to study wheelchair and seat

loading during frontal impact ${ }^{30,32,39-41}$ and rear impact conditions ${ }^{42-44}$. These studies used validated computer simulation models to investigate loading patterns imposed by impact to aid in the design of crashworthy pediatric and adult wheelchairs. Therefore, the purpose of this study was to develop a validated computer simulation model of a commercial, adult-occupied, manual wheelchair in LATV emergency braking maneuver. This model will serve as a valuable tool to investigate wheelchair and WTORS loading in low-g environment, which can aid in the design and development of WTORS suitable for LATV transit.

\section{Methodology}

The intent of this study was to develop and validate a model to simulate a LATV emergency maneuver for the purposes of estimating WTORS and wheelchair loading. A manual wheelchair was chosen because manual wheelchairs have almost twice the frequency of instability in LATVs when compared to power wheelchairs ${ }^{38}$. A widely used commercial manual wheelchair with common options was selected. The wheelchair 
(Quickie 2, Sunrise Medical) used in this study was an ultra-light, X-braced, manual wheelchair with the transit option (conforming to the ISO 7176-19 standard "Wheeled mobility devices for use as seats in motor vehicles" ${ }^{28}$ ). The wheelchair was adjusted to properly fit the ATD and featured a rigid seatback and a 2.8 in contoured seat cushion. The wheelchair passenger was represented by a wheelchair-seated, $50^{\text {th }}$ percentile Hybrid II anthropomorphic testing device (ATD- $74.5 \mathrm{~kg}$,). The ATD abdominal insert was removed to better simulate passenger kinematics in low-g environment ${ }^{37}$. Figure 1 illustrates the test set up.
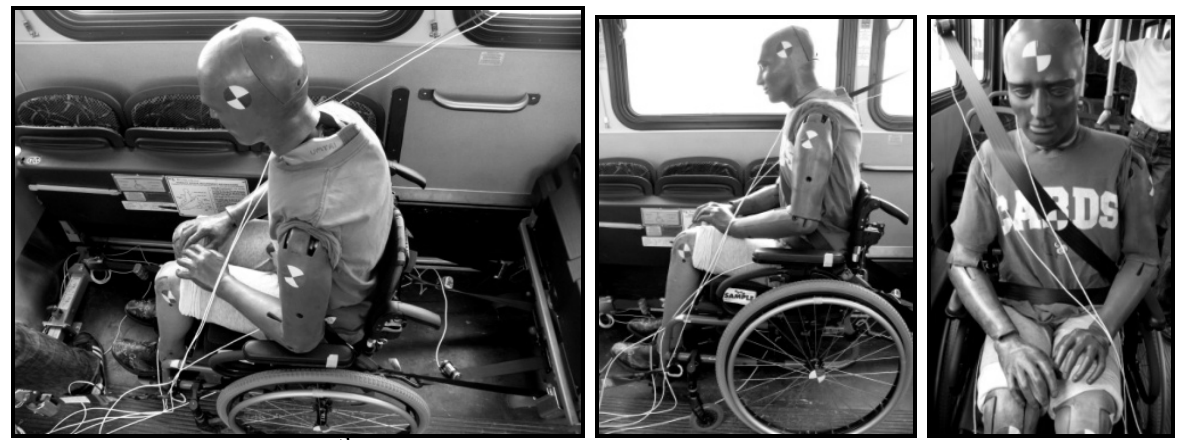

Figure 1. Test setup of $50^{\text {th }}$ percentile ATD seated in manual wheelchair with four tiedowns and lap and shoulder belt applied. Low-g accelerometers were mounted in the ATD head and chest, the wheelchair CG, as well as on the LATV floor (at securement station). Load cells were used to measure webbing tension in the tiedowns and lap and shoulder belts.

The wheelchair was secured at the securement station on the right side of the LATV (previous experience showed that wheelchair passengers use the right securement station). The securement station within the LATV conformed to ADA regulation with respect to size (measured size: 66 in long by 36 in wide), and featured a four-point, straptype, retractor-style WTORS (shoulder belt: SURE-LOK, FE200936M-AMS, lap belt: SURE-LOK, AL701060M-AMS, rear tiedowns: SURE-LOK, DAL 3300, front tiedowns: AL700963S-AMS) that were used to secure the wheelchair and restrain the occupant. 
The wheelchair-seated passenger represented by the ATD was instrumented with low-g, tri-axial accelerometers positioned at the head center of gravity $(\mathrm{CG})$ and inside the thorax instrumentation cavity, while the wheelchair was equipped with a tri-axial accelerometer at its CG. An additional tri-axial accelerometer was mounted on the floor of the LATV securement station (geometric center). All of the accelerometers were $0 \pm 6 \mathrm{~g}$ manufactured by Freescale Semiconductors, Inc. (Model \# MMA7260Q). The WTORS were instrumented with three-bar belt load cells (Denton Corp.), measuring tension forces in the webbing during testing. The load cells were calibrated up to $60 \mathrm{lb}$ at $5 \mathrm{lb}$ increments and zeroed prior to testing. WTORS retractors determined the pretension in all webbing. All acceleration and loading data was acquired at $500 \mathrm{~Hz}$ using a portable on-board customized data acquisition system. To minimize noise, this data was filtered using a Butterworth low pass filter. Fourier Transform revealed an appropriate cutoff frequency of 50Hz. Multiple views video (side and front view) of the securement station were captured using digital cameras at 30 frames/sec. High contrast markers were placed on the wheelchair and ATD to track kinematic response.

The LATV emergency combination braking/left-turn maneuver was performed in a parking lot, closed-off to outside traffic. The reason the LATV emergency braking while turning left (combination braking/left-turn) maneuver was chosen for model development and validation is that it produced the highest LATV acceleration compared to other LATV emergency maneuvers (braking, acceleration, turning, braking while turning right, and abrupt lane changes). The acceleration pulse recorded during the LATV emergency combination braking/left-turn maneuver that was used as input for our model is shown in Figure 2. 


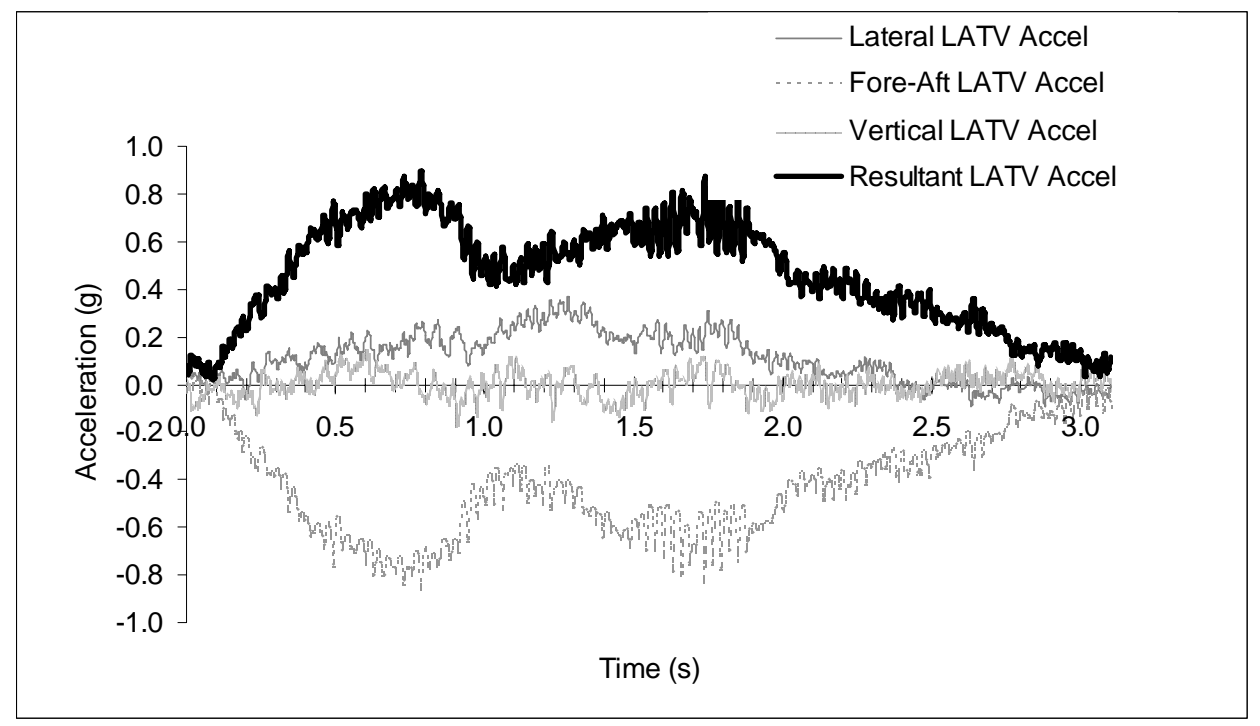

Figure 2. Experimental LATV acceleration pulse during a LATV emergency combination braking/left-turn maneuver.

The model was developed in MADYMO ${ }^{\mathrm{TM}}$ (MAthematical DYnamic MOdeling), which is an advanced software engineering tool developed by TNO (Delft, Netherlands). $\mathrm{MADYMO}^{\mathrm{TM}}$ has combined capabilities of finite element and rigid multi-body modeling. Multi-body modeling can be used for the simulation of gross motion of systems of bodies connected by kinematic joints, while finite element techniques can be applied to simulate structural behavior. While rigid bodies within $\mathrm{MADYMO}^{\mathrm{TM}}$ are assigned inertial properties, they are often "encased" by ellipsoids with geometric specifications; this gives the body a certain "shape." Ellipsoids can then interact with the surrounding ellipsoids and their respective bodies. A model can consist of rigid bodies, finite element bodies, or a combination of the two ${ }^{45}$.

The wheelchair frame, wheels, seat, and seatback were modeled in MADYMO ${ }^{\mathrm{TM}}$ using rigid multi-body ellipsoids. The masses of these wheelchair components were measured and their moments of inertia were determined; both were incorporated into the 
model. In the case of the wheelchair frame, frame tubing inertial properties were lumped together and represented in one body. The moments of inertia of individual frame components were combined using the parallel axis theorem while their masses were simply summed and represented in the model with a single body having the inertial properties of the entire wheelchair frame.

A validated ellipsoid ATD representing the $50^{\text {th }}$ percentile male Hybrid II was imported from the MADYMO ${ }^{\mathrm{TM}}$ TNO database. The 50th percentile ellipsoid ATD has 37 rigid bodies connected by numerous revolute, translational, revolute/translational, and spherical joints. The ATD segment inertial properties, joint and segment stiffness, as well as additional properties have been measured quasi-statically ${ }^{46}$. This Hybrid II ellipsoid ATD has been validated by TNO using a series of tests, including a frontal impact sled test $(48 \mathrm{~km} / \mathrm{h}, 20 \mathrm{~g})^{46}$. The model ATD was modified by removal of abdominal ellipsoids (all ellipsoids in the "abdomen multi-body group") to match the experiment ATD. Figure 3 shows the wheelchair model and the imported 50th percentile male Hybrid II ATD. 


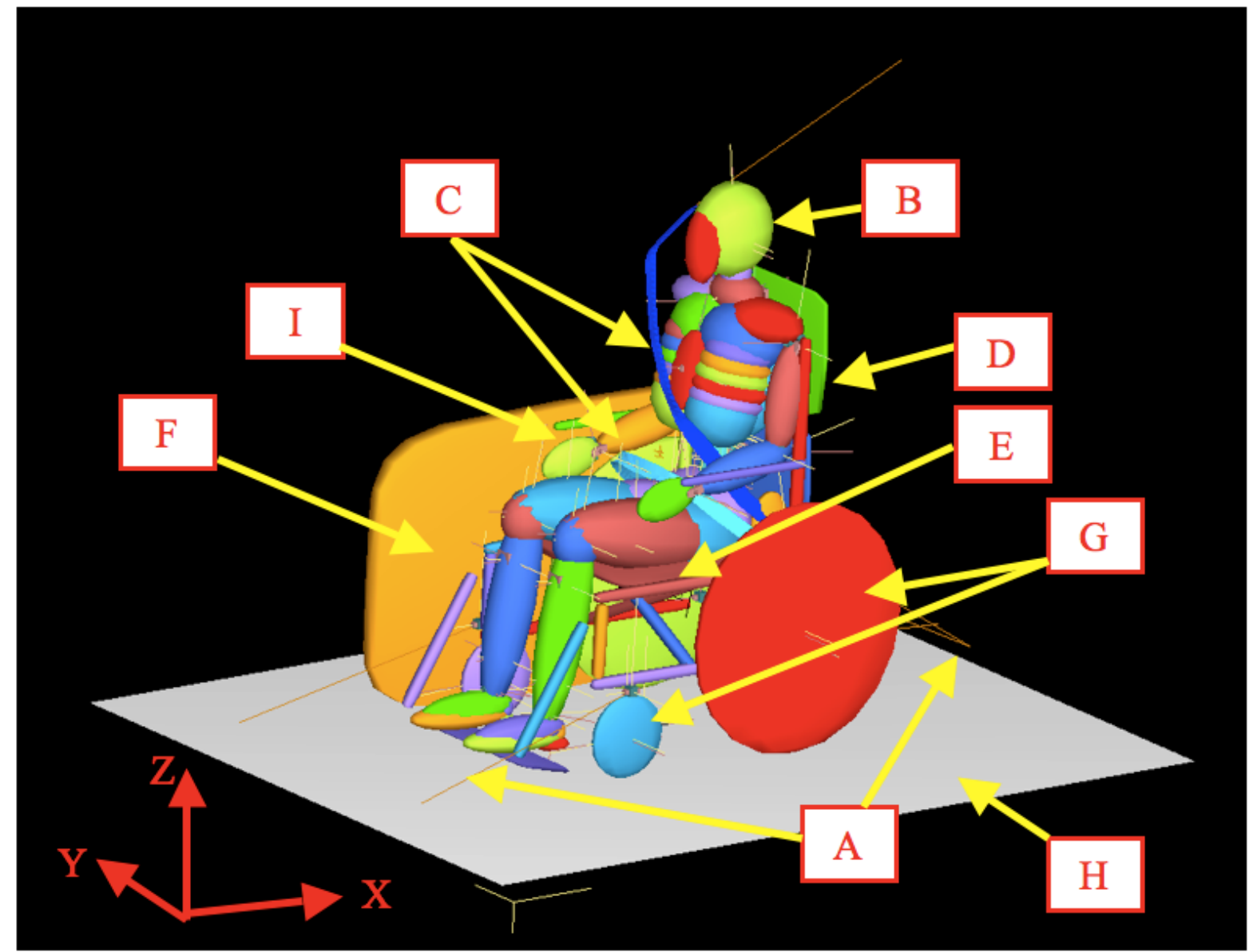

Legend:

A - front and rear tiedowns

B - 50th percentile male Hybrid II ATD representing wheelchair user

$\mathrm{C}$ - lap and shoulder belts

$\mathrm{D}$ - wheelchair seatback

E - wheelchair seat

$\mathrm{F}$ - wheelchair frame

$\mathrm{G}$ - front and rear wheels

$\mathrm{H}$ - LATV and LATV securement station

I - folding seat

Figure 3. LATV wheelchair model (forward- facing) with 50th percentile ATD.

The wheelchair in the model was secured with front and rear tiedowns modeled as belt segments [A]. The 50th percentile Hybrid II ATD [B] was imported from a TNO database into the MADYMO ${ }^{\mathrm{TM}}$ model. The lap and shoulder belts [C] were modeled with finite element belts, while the wheelchair seatback [D] was modeled with ellipsoid surfaces. The wheelchair seat [E] was modeled with an ellipsoid surface and translational joint, allowing fore-aft displacement along $\mathrm{X}$-axis. The wheelchair frame $[\mathrm{F}]$ was 
represented by 1 body and 16 ellipsoid surfaces, while the individual wheels [G] were modeled using single surface ellipsoids. The LATV (including the suspension characteristics) and LATV securement station $[\mathrm{H}]$ were represented using a plane surface and a rotational-translational joint to allow tilting in the sagittal plane. A surface ellipsoid was used to represent the folded seat [I], right of the securement station.

Validation of the model involved an initial visual comparison of the model with experimental test videos to assess the gross wheelchair and ATD kinematics. Secondly, time history curves for all model outcome measures (Table 1) were compared to those in the experimental test.

\begin{tabular}{|l|l|}
\cline { 2 - 2 } \multicolumn{1}{c|}{} & Outcome measure \\
\hline 1 & Rear tiedown loading \\
\hline 2 & Shoulder belt loading \\
\hline 3 & Wheelchair resultant acceleration \\
\hline 4 & ATD resultant head acceleration \\
\hline 5 & ATD resultant chest acceleration \\
\hline
\end{tabular}

Note: Front tiedown and lap belt loading were not used in the validation process, because they were negligible due to the wheelchair and ATD dynamics.

Table 1. List of outcome measures used in model validation.

For each outcome measure, the simulation output was graphically over-laid onto the experimental history for visual comparison of general curve shapes. This visual comparison between the time histories was used as a primary means to determine model validity. Finally, four statistical assessments were used to quantify and further test agreement between experimental and simulation outcome measure time histories. The statistical tests were conducted using CurveAnalyzer statistical software ${ }^{47}$. A summary 
of the tests and test acceptance criteria used in the statistical analysis are shown in Table 2 below:

\begin{tabular}{|l|l|l|l|l|}
\hline $\begin{array}{l}\text { Statistical } \\
\text { Test }\end{array}$ & $\begin{array}{l}\text { Mean Value } \\
\text { Ratio }\end{array}$ & $\begin{array}{l}\text { Correlation } \\
\text { Coefficient }\end{array}$ & $\begin{array}{l}\text { Std. Deviation } \\
\text { of Residuals }\end{array}$ & $\begin{array}{l}\text { Peak } \\
\text { Value }\end{array}$ \\
\hline Criteria & $L=0.80-1.20$ & $r=0.8$ & $\sigma_{d} \leq 0.2 P_{s}$ & $\begin{array}{l}(+/-20 \%, \\
+/-0.25 \mathrm{~s})\end{array}$ \\
\hline
\end{tabular}

Table 2. Summary of Statistical Tests and Test Criteria for Model Validation Assessment.

The statistical tests, test criteria, and suggested validity ranges were chosen based on a previously conducted study by Pipcorn et. al. ${ }^{48}$. Historically, statistical analysis has not been conducted to assess validation in occupant crash simulation models. Pipcorn, et al. attempted to quantify the previous subjective approaches to model validation through the use of statistical analysis evaluating various aspects of time history curves. The four statistical tests used to assess agreement between the model and experiment in our study are shown in Table 2 and are described below.

\section{Mean Value Ratio test}

The mean value ratio $(L)$ of the time histories was computed using

$$
\begin{aligned}
& L=\frac{\bar{S}_{\exp }}{\overline{S_{\bmod e l}}}(1) \\
& \bar{s}=\sum_{i=1}^{n} \frac{f_{i}}{n}
\end{aligned}
$$


where $\bar{s}$ is the time history mean, $f_{i}$ is the amplitude of the time history to be compared, $i$ is each point in time, and $n$ is the number of samples. The time history mean value from the experiment was divided by the time history mean from the model, with a result near unity indicating that the two time histories are likely to be the same or related. The suggested validity range was $L=0.80-1.20$.

\section{$\underline{\text { Correlation test }}$}

The correlation coefficient $(r)$ was calculated using

$$
r=\frac{\sum_{i=1}^{n}\left(f_{i}-\bar{f}\right)\left(g_{i}-\bar{g}\right)}{\sqrt{\sum_{i=1}^{n}\left(f_{i}-\bar{f}\right)^{2} \sum_{i=1}^{n}\left(g_{i}-\bar{g}\right)^{2}}}
$$

where $f_{i}$ is the amplitude of the experiment time history, $g_{i}$ is the amplitude of the model

time history, $i$ is each point in time, $\bar{f}_{\text {is the experiment time history mean, }} \bar{g}_{\text {is the }}$ model time history mean. The suggested validity range was $r>0.80$.

\section{$\underline{\text { Standard Deviation of Residuals test }}$}

Since the experiment data and model data was obtained at the same frequency, each time history data point in the experiment had a corresponding time history data point in the model. The difference between the two data points is the residual. Standard deviation of residuals $\left({ }^{\sigma_{d}}\right)$ was calculated using 


$$
\sigma_{d}=\sqrt{\frac{n \sum_{i=1}^{n} d_{i}{ }^{2}-\left(\sum_{i=1}^{n} d_{i}\right)^{2}}{n(n-1)}}
$$

where $d_{i}$ is the residual, $i$ is each point in time, and $n$ is the number of data points. The

suggested limit for $\sigma_{d}$ was $20 \%$ of the peak value from the experiment time history $\left(P_{s}\right)$.

\section{$\underline{\text { Peak Value test }}$}

When comparing time history peaks from the model to those from the experiment, the peak value assessment was not only considered the magnitude of each peak, but also when this peak occurs. The proposed method was to establish an acceptable boundary region around the experiment peak $\left(P_{s}\right)$. The lower bound of the region was $80 \%$, while the upper bound was $120 \%$ of the experiment peak. The right and left bounds of the time region were $+/-0.25 \mathrm{~s}$ from when the experiment peak occurred. The model peak needed to fall within the acceptance region for the peak value to be considered valid. Only the primary peak in each time history was evaluated, while secondary peaks were ignored.

\section{Results}

The initial validation was done by comparing wheelchair and ATD gross kinematics from the experiment to those from the model. Figure 4 shows a typical frame sequence during the experiment (top) and the corresponding frames generated from the model simulation (bottom). The comparison showed reasonably good correlation of gross wheelchair and occupant kinematics, suggesting initial validation of the model. 


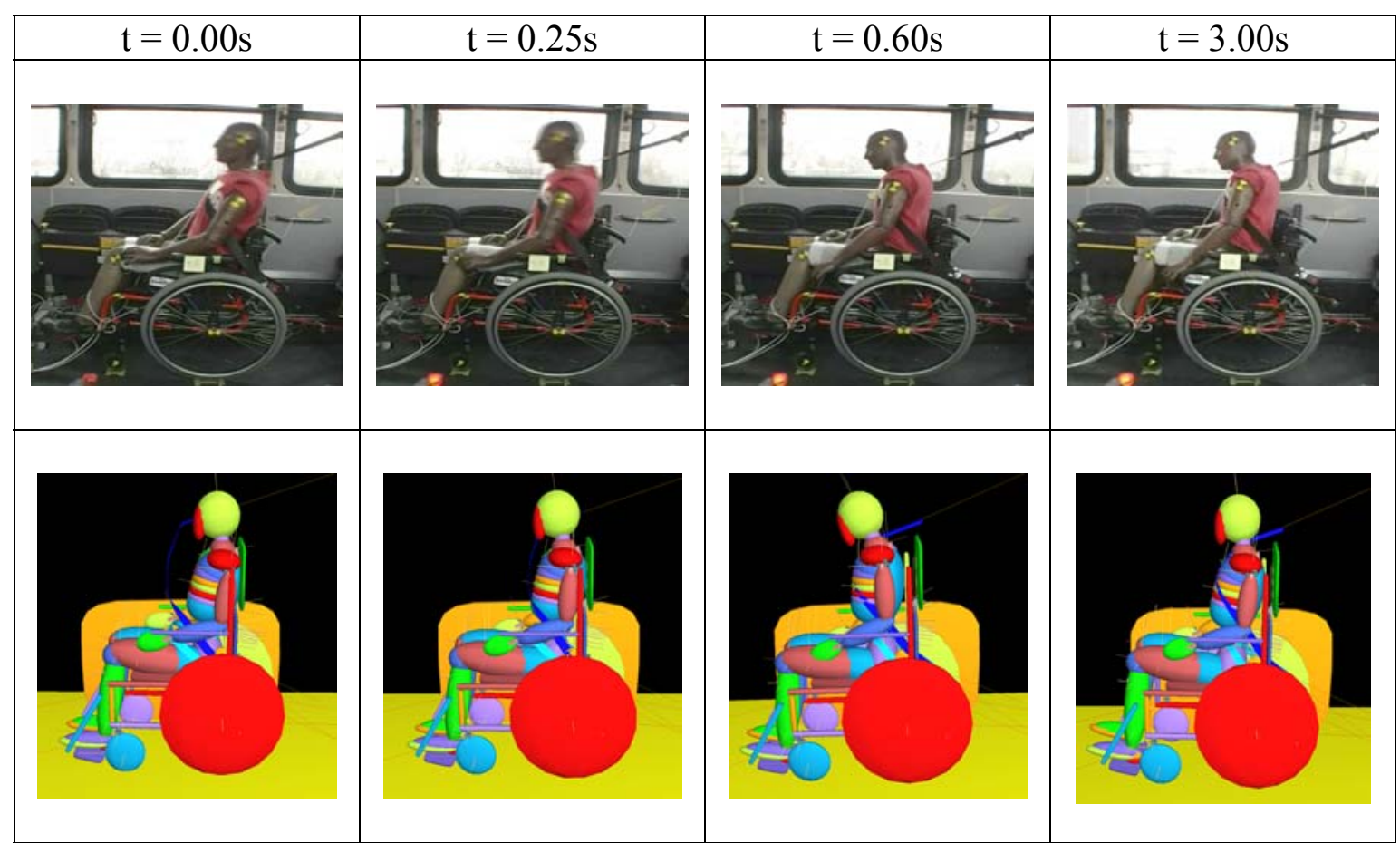

Figure 4. Frame Sequence of Experimental Test (Top) and Computer Simulation Model (Bottom).

The model validation was assessed with respect to the outcome measures from Table 1. Figures 5-10 show the time history comparisons between the experiment and model for the outcome measures.

Figure 5 shows a comparison of the model right rear tiedown (RRTD) loading to the experimental RRTD loading. The model RRTD loading curve follows the experimental RRTD loading curve well and has a peak value of $45 \mathrm{lb}$ at $0.85 \mathrm{~s}$. The experimental RRTD peak was also $45 \mathrm{lb}$ at $0.85 \mathrm{~s}$. 


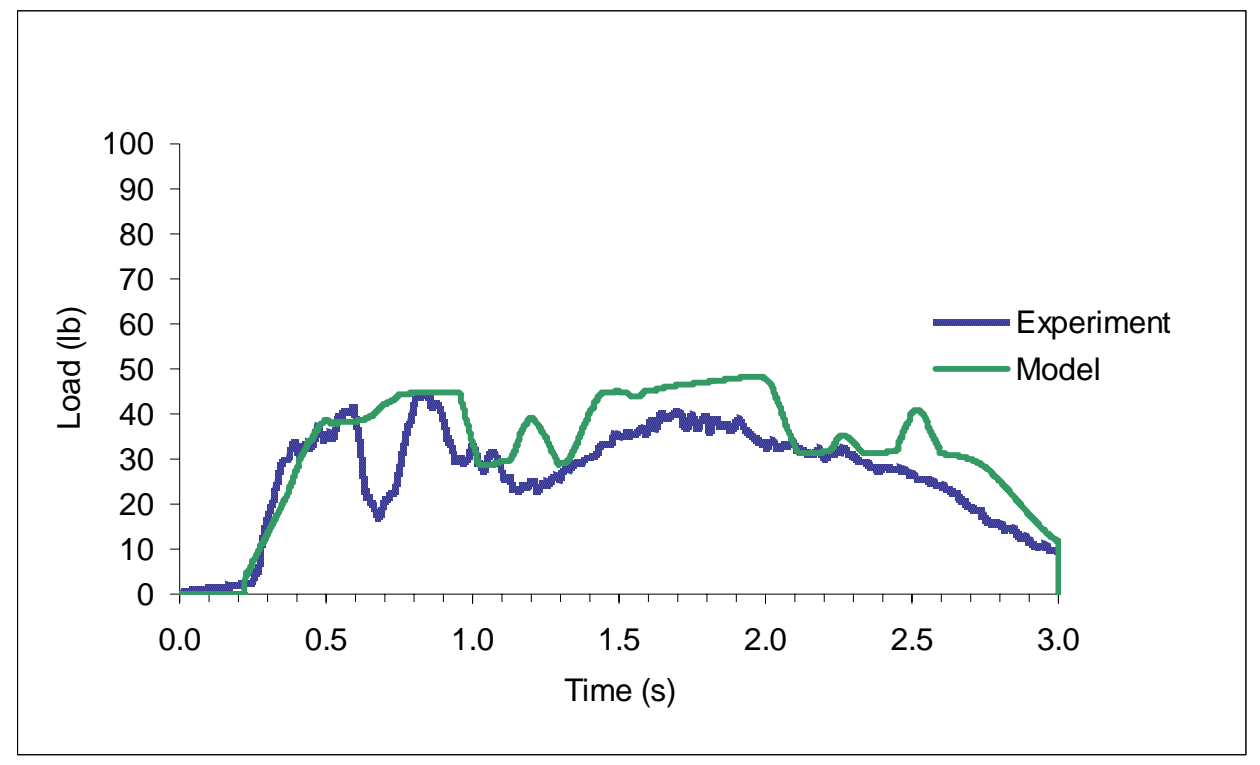

Figure 5. Right rear tiedown loading: model (solid line) vs. experiment (dashed).

Figure 6 shows the model left rear tiedown (LRTD) loading in comparison to the experimental LRTD loading. The model LRTD loading curve follows the experimental LRTD loading curve reasonably well and has a peak value of $39 \mathrm{lb}$ at $0.75 \mathrm{~s}$. The experimental LRTD peak was $38 \mathrm{lb}$ and occurs $0.25 \mathrm{~s}$ before the model LRTD peak.

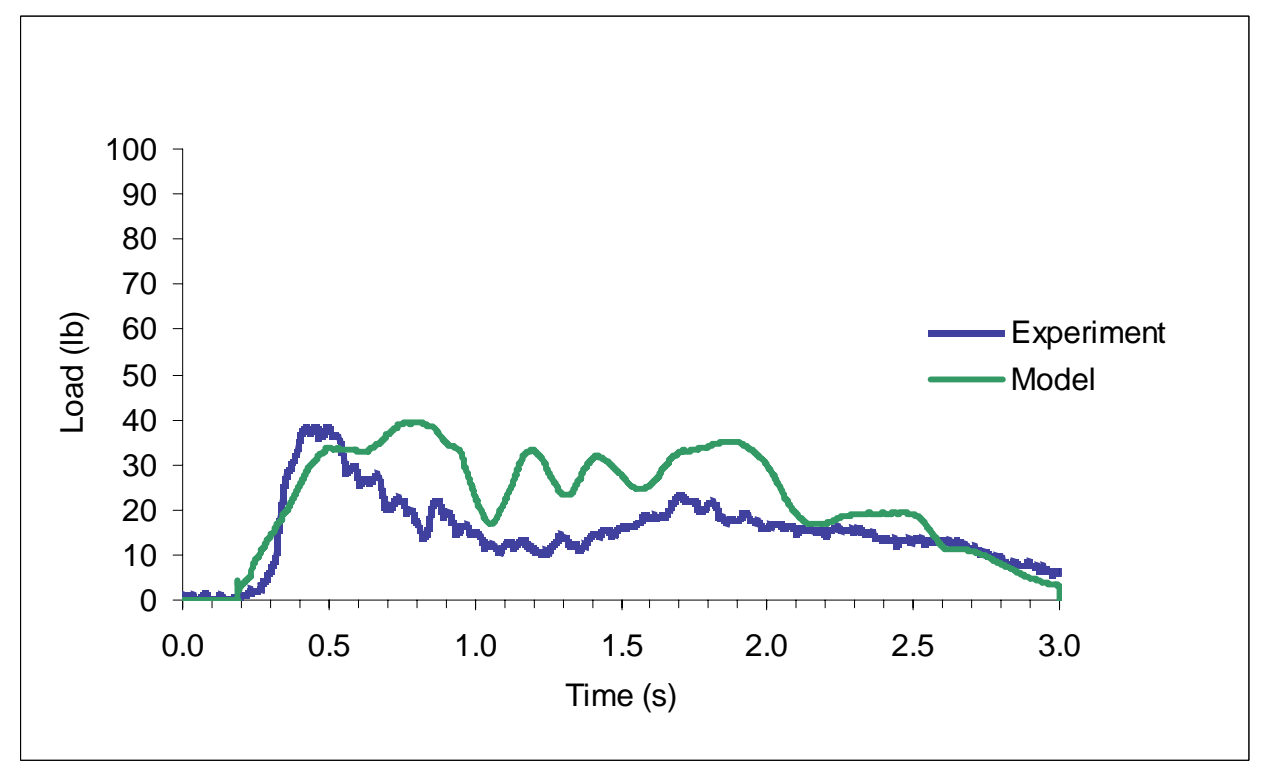

Figure 6. Left rear tiedown loading: model (solid line) vs. experiment (dashed). 
Figure 7 shows the model shoulder belt (SB) loading in comparison to the experimental SB loading. The model SB loading curve generally follows the experimental SB loading curve and has a peak value of $95 \mathrm{lb}$ at $0.55 \mathrm{~s}$. The experimental SB peak was $105 \mathrm{lb}$ occurred at $0.59 \mathrm{~s}$.

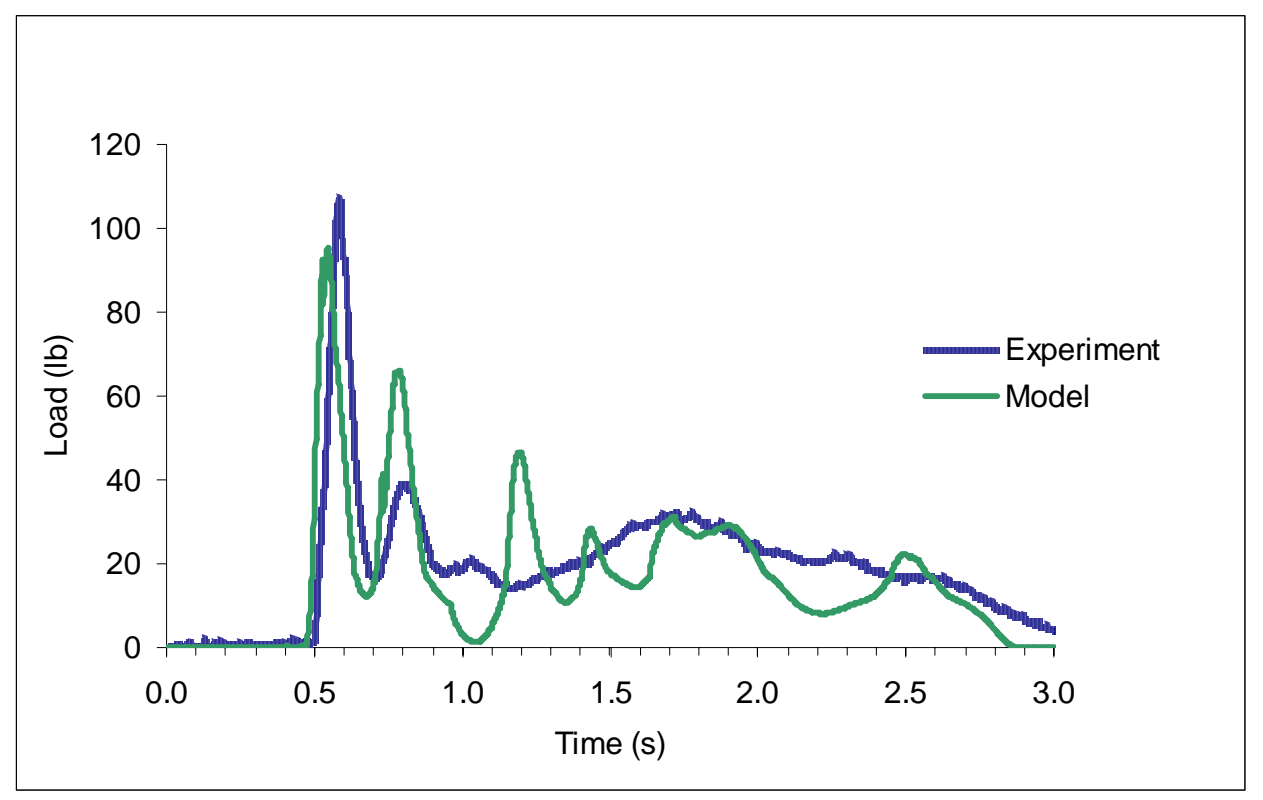

Figure 7. Shoulder belt loading: model (solid line) vs. experiment (dashed).

Figure 8 shows a comparison of the model wheelchair resultant acceleration to experimental wheelchair resultant acceleration. The model wheelchair resultant acceleration curve generally follows the experimental wheelchair resultant acceleration curve and has a peak value of $1.19 \mathrm{~g}$ at $0.55 \mathrm{~s}$. The wheelchair resultant acceleration peak was $1.14 \mathrm{~g}$ and occurred at $0.63 \mathrm{~s}$. 


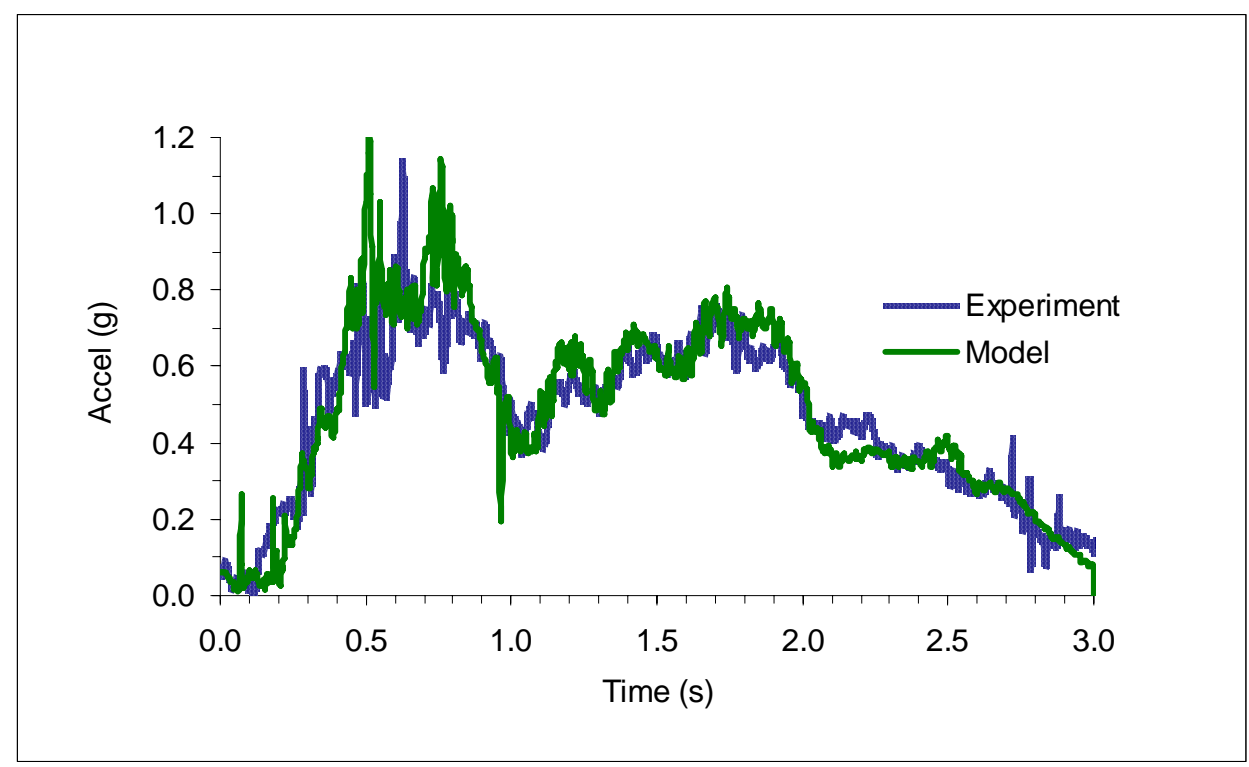

Figure 8. Wheelchair resultant acceleration: model (solid line) vs. experiment (dashed).

Figure 9 shows a comparison of the model ATD chest resultant acceleration to experimental ATD chest resultant acceleration. The model ATD chest resultant acceleration curve generally follows the experimental ATD chest resultant acceleration curve and has a peak value of $2.63 \mathrm{~g}$ at $0.55 \mathrm{~s}$. The ATD chest resultant acceleration peak was $2.65 \mathrm{~g}$ and occurred at $0.58 \mathrm{~s}$. 


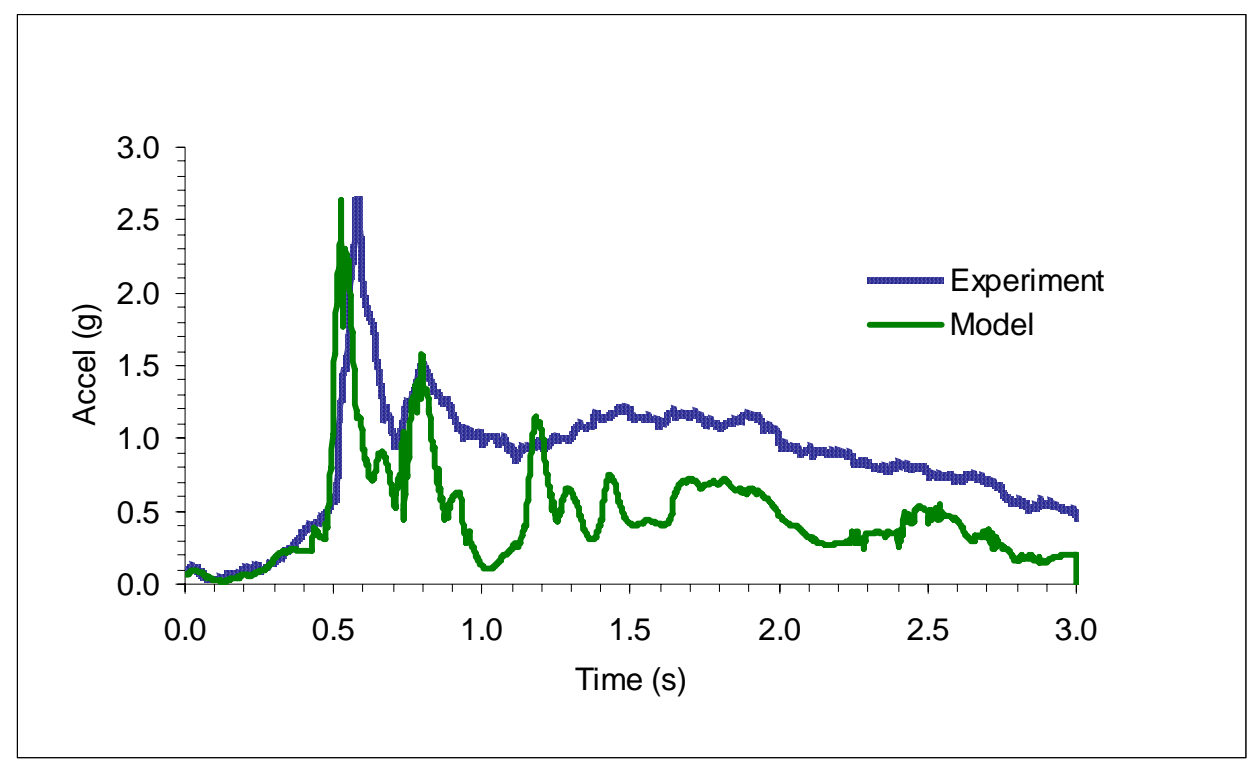

Figure 9. ATD chest resultant acceleration: model (solid line) vs. experiment (dashed).

Figure 10 shows a comparison of the model ATD head resultant acceleration to experimental ATD head resultant acceleration. The model ATD head resultant acceleration curve generally follows the experimental ATD head resultant acceleration curve and has a peak value of $3.33 \mathrm{~g}$ at $0.58 \mathrm{~s}$. The ATD head resultant acceleration peak was $3.83 \mathrm{~g}$ and occurred at $0.59 \mathrm{~s}$. 


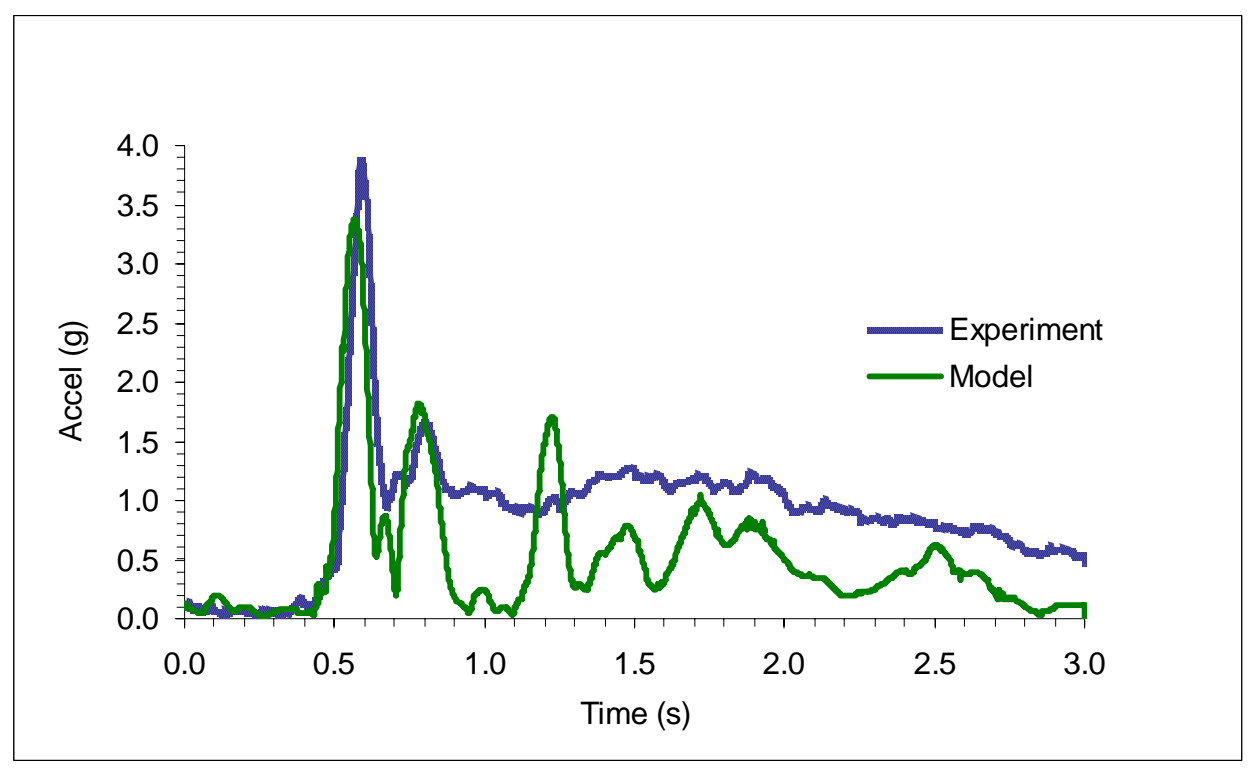

Figure 10. ATD head resultant acceleration: model (solid line) vs. experiment (dashed).

Table 3 below shows a summary of the statistical test results, comparing the model to the experiment for each outcome measure. Although the LRTD loading, and ATD resultant chest and head acceleration time histories met two out of four statistical test criteria, the other outcome measure time histories met at least three out of four statistical test criteria. The resultant wheelchair acceleration and RRTD loading time histories met all four statistical criteria. Statistical analysis was performed for the entire LATV acceleration pulse duration (0.00-3.00 s). 


\begin{tabular}{|c|c|c|c|c|c|}
\hline & \multicolumn{4}{|c|}{ Statistical Test } & \\
\hline & $\begin{array}{l}\text { Mean } \\
\text { Value } \\
\text { Ratio }\end{array}$ & $\begin{array}{l}\text { Correlation } \\
\text { Coefficient }\end{array}$ & $\begin{array}{c}\text { Std. } \\
\text { Deviation } \\
\text { of Residuals }\end{array}$ & Peak Value & \\
\hline Criteria & $\mathrm{L}=0.20$ & $\mathrm{r}=0.8$ & $\mathrm{~s}=0.2$ & $\begin{array}{l}+/-20 \% \\
+/-0.25 \mathrm{~s}\end{array}$ & $\begin{array}{c}\text { \# of Tests } \\
\text { Meeting } \\
\text { Criteria } \\
\end{array}$ \\
\hline $\begin{array}{c}\text { Right Rear } \\
\text { Tiedown Loading }\end{array}$ & $\begin{array}{l}\sqrt{ } \\
x=1.1961\end{array}$ & $\begin{array}{l}\sqrt{ } \\
\mathrm{r}=0.88903\end{array}$ & $\begin{array}{l}\sqrt{ } \\
\mathrm{s}=0.0026\end{array}$ & $\sqrt{ }$ & 4 \\
\hline $\begin{array}{c}\text { Left Rear } \\
\text { Tiedown Loading }\end{array}$ & $\underset{x=1.4842}{X}$ & $\underset{r=0.6941}{X}$ & $\begin{array}{c}\sqrt{ } \\
\mathrm{s}=0.0047\end{array}$ & $\sqrt{ }$ & 2 \\
\hline $\begin{array}{l}\text { Shoulder Belt } \\
\text { Loading }\end{array}$ & $\begin{array}{c}\sqrt{ } \\
\mathrm{x}=0.8577\end{array}$ & $\underset{r=0.7283}{X}$ & $\begin{array}{c}\sqrt{ } \\
\mathrm{s}=0.0013\end{array}$ & $\sqrt{ }$ & 3 \\
\hline $\begin{array}{c}\text { Wheelchair } \\
\text { Resultant } \\
\text { Accel }\end{array}$ & $\begin{array}{c}\sqrt{ } \\
x=1.0341\end{array}$ & $\begin{array}{c}\sqrt{ } \\
\mathrm{r}=0.919\end{array}$ & $\begin{array}{c}\sqrt{ } \\
\mathrm{s}=0.0656\end{array}$ & $\sqrt{ }$ & 4 \\
\hline $\begin{array}{c}\text { ATD Chest } \\
\text { Resultant Accel }\end{array}$ & $\underset{x=0.5423}{X}$ & $\underset{\mathrm{r}=0.681}{\mathrm{X}}$ & $\begin{array}{c}\sqrt{ } \\
\mathrm{s}=0.0456\end{array}$ & $\sqrt{ }$ & 2 \\
\hline $\begin{array}{c}\text { ATD Head } \\
\text { Resultant Accel }\end{array}$ & $\underset{x=0.5754}{X}$ & $\underset{\mathrm{r}=0.7628}{\mathrm{X}}$ & $\begin{array}{c}\sqrt{ } \\
s=0.0339\end{array}$ & $\sqrt{ }$ & 2 \\
\hline
\end{tabular}

Table 3. Statistical comparison summary of model and experimental outcome measure time histories.

In summary, the model was considered validated. Firstly, the gross kinematics of the wheelchair and ATD in the model match those from the experiment. The outcome measure time histories follow their experimental counterparts well. Statistical analysis revealed that all model outcome measure time histories meet at least two out of four 
statistical test criteria when compared to the experimental time histories, with the wheelchair resultant acceleration meeting all four statistical test criteria.

\section{Discussions and Conclusions}

\section{$\underline{\text { Computer Model Development }}$}

The wheelchair and occupant dynamics during a braking maneuver differ from those during an acceleration maneuver. During a braking maneuver the ATD primarily loads the occupant restraints as it moves forward, while the rear tiedowns primarily secure the wheelchair by preventing forward excursion. The front tiedowns and seatback are loaded minimally. During an acceleration maneuver however, the front tiedowns serve as the primary means of maintaining wheelchair securement and preventing rearward excursion, while the seatback serves as the primary means of restraining or containing the occupant in the wheelchair seat. Our model may not be a good predictor of wheelchair/occupant dynamics, and wheelchair/WTORS loading if subjected to an LATV acceleration maneuver.

The LATV maneuver input for our model is a combination of braking while making a left turn. The wheelchair and passenger dynamics in this case differ somewhat from traditional braking maneuver dynamics as the wheelchair and occupant exhibit additional lateral excursions to the right while still moving forward with the ATD loading the occupant restraints and rear tiedowns securing the wheelchair. The additional lateral wheelchair excursion to the right caused wheelchair contact with the folded seat (under the window) at the right of the securement station (Figure 1 in experiment, Figure 3 in model). Our model replicated these wheelchair and ATD dynamics. 
The $50^{\text {th }}$ percentile Hybrid II ellipsoid ATD was developed by the software manufacturer, TNO, and was imported from the provided MADYMO ${ }^{\mathrm{TM}}$ database. TNO validated this particular Hybrid II ellipsoid model using frontal impact crash testing (48 $\mathrm{km} / \mathrm{h}, 20 \mathrm{~g}$ ), but the validation process involved only two sled tests and did not statistically compare the ATD model to the sled test outcome measures, but relied on 'engineering judgment' to confirm validity ${ }^{46}$. We also modified the ATD by removing abdominal ellipsoids.

At first, the wheelchair in our model was developed as simplistically as possible, but increased in complexity with additional joints, bodies, and ellipsoids during the validation process.

\section{Computer Model Validation}

The initial validation of the computer model was based on assuring that the wheelchair and ATD kinematics in the model were visually analogous to those in the experiment. This process required that key events occurred at the same time and that both wheelchair and ATD excursions in the model visually matched those from the experiment. The frame sequence in Figure 4 shows that the model kinematics (bottom) reasonably matched the experiment kinematics (top) at various points in time during the LATV emergency combination braking/left-turn maneuver. The first frame at $\mathrm{t}=0.00 \mathrm{~s}$ indicates the beginning of the deceleration pulse and therefore the point in time when the maneuver starts. At $\mathrm{t}=0.25 \mathrm{~s}$, the wheelchair begins to move forward and to the right loading the rear tiedowns and making contact with the folded seat right of the securement station. The ATD loads the SB around $0.60 \mathrm{~s}$ with maximum ATD chest and head 
resultant accelerations also occurring around this time. The combination braking/left turn maneuver is complete around $3.00 \mathrm{~s}$.

Overall the model kinematics of the wheelchair and ATD reasonably match those of the experiment. Ultimately this approach in model validation should increase the likelihood that loads and accelerations between the model and experiment are similar in terms of magnitude and time of peak occurrence.

Next, the key model outcome measure time histories were validated. Figure 5 shows a relatively good fit of the model RRTD loading with respect to the experimental RRTD loading time history. The only point in the time history when the model slightly deviates from the experimental RRTD loading is near $0.70 \mathrm{~s}$. The RRTD loading time history curve in the model does not unload the way it does in the experiment. However, the model's RRTD loading was considered validated against the experiment, since all four statistical test criteria have been satisfied (Table 3).

The model LRTD loading time history curve follows its experimental counter part reasonably well, but not as closely as the RRTD loading outcome measure. The model LRTD loading peak occurs $0.25 \mathrm{~s}$ after the experiment LRTD loading peak and does not unload as rapidly. One possible reason for this may be that the rear tiedown geometry is different for the LRTD compared to the RRTD. With respect to the sagittal plane, the RRTD makes a $4^{\circ}$ angle in comparison to a $30^{\circ}$ angle for the LRTD. Even though this difference in rear tiedown geometry was replicated in the model, it may explain the minor variation in model vs. experimental LRTD loading. Another factor that could contribute to LRTD loading variation in the model vs. experiment is the amount of LRTD pretension. Statistical analysis revealed that two of four statistical test criteria were 
satisfied. Most importantly, the model LRTD peak matched the experimental LRTD peak.

Loading of the SB was the only occupant restraint belt considered as an outcome measure for validation, because lap belt loading was negligible in experimental testing, as it is in the model. During the combination braking/left-turn maneuver, the SB alone keeps the ATD from moving forward. Figure 7 shows reasonable agreement between the model and experiment SB loading time histories. The SB load peak generated from the model is lower than in the experiment by $10 \mathrm{lb}$. This may be due to the ATD dynamics in the lowg environment. The imported model ATD was validated by TNO in a high-g crash environment. Nevertheless, the statistical analysis showed that the SB loading outcome measure met three out of four statistical test criteria and can be considered validated. Figure 8 shows model and experiment wheelchair resultant acceleration time histories following relatively closely. The model wheelchair resultant acceleration peak is only $0.05 \mathrm{~g}$ higher than, and occurs $0.08 \mathrm{~s}$ before its experimental counterpart. As all four statistical test criteria have been satisfied, the resultant wheelchair acceleration time history can be considered validated.

We also considered both the ATD head and chest resultant accelerations to be validated, even as only two of four test criteria are satisfied in a comparison of the model to the experiment. In both cases the model and experiment time history shapes and peaks match well from $0.00 \mathrm{~s}$ until approximately $0.85 \mathrm{~s}$. This can be considered the most important time period for ATD head and chest accelerations as this is when the ATD contacts the SB and the ATD accelerations peak. Differences between model and 
experiment after $0.85 \mathrm{~s}$ may be attributable to model ATD behavior under longer duration low-g conditions.

\section{Comparison to previous wheelchair-occupant models}

Overall, the model validation in this study can be considered relatively robust. Review of the literature showed that early computer model validations were limited. Even though Paskoff's rear impact model of a $50^{\text {th }}$ percentile ATD seated in an adult manual wheelchair was able to describe occupant kinematics and forces to some extent, the model was not validated in rear impact using dynamic crash testing ${ }^{43}$.

Bertocci, et al. ${ }^{39}$ validated their model of a mid-sized ATD seated in a commercial powered wheelchair subjected to frontal impact utilizing data from two sled tests. First, the gross motions of the occupant and wheelchair in the model were compared to those from sled testing. Peak values and general time history profiles of the following model parameters were compared to sled test results: WTORS loads, resultant head, chest, and wheelchair accelerations, along with head and front wheel excursions. Only limited comparisons were employed in the validation process, including a visual comparison of time histories, peak value comparisons and range percent difference between the model and sled test outcome measures.

Leary and Bertocci's first step in the validation process of their frontal impact model of a $50^{\text {th }}$ percentile ATD in an adult manual wheelchair was a visual comparison of gross occupant and wheelchair kinematics to sled test videos ${ }^{32}$. The time histories of the following various outcome measures were then also compared to those in the sled test: shoulder belt loading, lap belt loading, (rear) tiedown loading, wheelchair acceleration, 
ATD chest acceleration, and ATD pelvic acceleration. Validation criteria were established as having "relatively similar" peak values and "profile fit" between simulation model and sled test. No statistical techniques were utilized for validation ${ }^{32}$.

A more recent frontal impact model of a pediatric wheelchair occupied by a 6year-old ATD by Ha and Bertocci featured a more rigorous validation method ${ }^{40}$. In the validation process, a visual comparison of the sled test videos and the model wheelchair and occupant kinematics was conducted ${ }^{40}$. The authors also compared the trends and peaks of outcome measure time histories generated from the model to those acquired during sled testing. The outcome measures used in the comparison were wheelchair acceleration, wheelchair rear tiedown forces, occupant restraint shoulder and lap belt forces, and ATD chest and head accelerations. Additionally, a comparison between the model and sled test peak horizontal excursions was conducted for the wheelchair, ATD knee, and ATD head in the forward direction. The statistical analysis used in the validation process consisted of determining percent of peak difference, Pearson's correlation coefficient (r), and performing a linear regression analysis of model and sled test peak outcome values. The statistical analysis results were compared to initially defined criteria and to those from studies conducted previously. The authors concluded that their model was valid and could be used as a foundation for studying injury risk associated with children traveling seated in wheelchairs and providing pediatric wheelchair design guidelines.

Two other recent models of a mid-sized ATD occupying an adult manual wheelchairs subjected to frontal and rear impact were developed by Dsouza and Bertocci ${ }^{41}$ and Salipur and Bertocci ${ }^{44}$, respectively. Both models underwent rigorous validation, 
which provided the basis for our model validation process. In the first step of this models' validation process, the model-predicted gross kinematics of wheelchair and ATD were visually compared to those recorded from high-speed sled test videos. Secondly, the models' predicted time histories of loading and acceleration data were super-imposed over respective sled test time history $\mathrm{min} /$ max corridors. Various model parameters were tuned until time history plots shared similar trends between the model and sled tests. Finally, both studies used statistical analysis to quantify the association between model and sled test outcome measures. The four statistical tests both models had in common were mean value ratio, peak value and time occurrence, correlation coefficient, and standard deviation of residuals. In both models, the mean sled test time history from each outcome measure was used in the statistical comparison. The authors concluded, in both cases, that their respective computer model was validated and had high predictability of the sled tests.

The validation process for our model of a wheelchair-occupant in a LATV emergency combination braking/left-turn maneuver is most similar to the model validation process used by Dsouza and Bertocci ${ }^{41}$ and Salipur and Bertocci ${ }^{44}$. Our study and the Dsouza and Bertocci, and Salipur and Bertocci studies utilized multiple statistical tests to quantify agreement between experimental and model outcome measure time histories. Other models lack a rigorous validation process, with the exception of the $\mathrm{Ha}$ and Bertocci ${ }^{40}$ model. 


\section{$\underline{\text { Limitations }}$}

There are limitations associated with using a single test condition for model validation. However the test condition was chosen to represent a common scenario, with a $50^{\text {th }}$ percentile ATD occupying a manual wheelchair. Further investigation is needed to study effects of other types of occupants and wheelchairs.

Our model is validated for LATV emergency braking maneuvers, but it may not be suitable for all other LATV maneuvers, such as acceleration or pure turning maneuvers. The ATD and wheelchair dynamics in our model are specific to low-g LATV braking/left turn maneuvers. LATV accelerations and pure turning maneuvers would produce different ATD and wheelchair dynamics that may not be accurately predicted by our model. Further research is needed to determine the model's performance in other LATV maneuvers.

Furthermore, our model validation was limited to only one experimental LATV emergency combination braking/left-turn maneuver. Additional experimental maneuvers would have provided a more robust model validation. However, limited resources (mainly LATV and LATV operator time) prevented us from collecting additional usable data to be included for model validation. Nonetheless, we consider our model a good predictor of ATD and wheelchair dynamics in LATV emergency combination braking/left-turn maneuvers.

Another limitation is the one type of manual wheelchair make and model used for the experiment and model development/validation; other manual wheelchairs may generate different loading and kinematics. Also, a many wheelchairs in vehicles have substantially different geometry and greater mass than the manual wheelchair modeled in 
this study. Additional limitations include the simplified wheelchair representation within the model, and the fact that model validation was limited to only one LATV maneuver without model verification.

There are also several limitations associated with the ATD. ATDs lack biofidelity and may not truly represent a wheelchair occupant. The $50^{\text {th }}$ percentile male Hybrid II ATD was intended for frontal impact crash environment (high-g), not low-g LATV emergency maneuvers. TNO's imported ATD model was also only validated for high-g (frontal impact) use, not low-g studies. We also modified the ATD by removing the abdominal ellipsoids.

The statistical analysis conducted to quantify validation was limited to four statistical tests; additional statistical tests may better demonstrate model validity or the lack there of. Finally, the validation of ATD and wheelchair acceleration time histories was limited to resultant values and the horizontal and vertical components were not considered individually. However, since the model is intended for study of wheelchair and WTORS loading, resultant accelerations should be sufficient to represent ATD and wheelchair kinematics.

\section{Future Work}

The model developed and validated in this study will be used to investigate effects of various parameters on wheelchair and WTORS loading under LATV emergency combination braking/left-turn maneuvers. Model parameters that could be varied in a parametric sensitivity analysis include front securement point height, ATD mass, wheelchair mass, wheelchair CG location, number of tiedowns, and LATV 
suspension stiffness. Gaining an understanding of how these and other parameters affect wheelchair and WTORS loading in LATV emergency braking maneuvers can help wheelchair and WTORS manufacturers design safer products.

\section{$\underline{\text { Conclusion }}$}

The computer model developed in this study simulated a LATV emergency combination braking/left-turn maneuver experienced by a commercial adult manual wheelchair occupied by a $50^{\text {th }}$ percentile male ATD. The model was successfully validated using visual comparison of wheelchair and ATD kinematics, comparison of key model and experimental outcome measure time histories, as well as statistical analyses to quantify the level of validity. Compared to most previous studies and models, the validation process in this study was relatively rigorous. The model in this study was determined to be validated and a reasonable predictor of LATV emergency combination braking/left-turn maneuver involving a manual wheelchair and a seated $50^{\text {th }}$ percentile ATD. The model will aid in providing guidelines for tiedown and wheelchair manufacturers so that wheelchairs and securement systems suitable for LATV emergency braking maneuvers can be designed. 


\title{
CHAPTER 7
}

\author{
PARAMETRIC SENSITIVITY ANALYSIS USING COMPUTER SIMULATION \\ MODEL OF AN ADULT MANUAL TRANSIT WHEELCHAIR WITH A SEATED \\ OCCUPANT IN A LARGE ACCESSIBLE TRANSIT VEHICLE UNDER \\ EMERGENCY BRAKING
}

\section{Overview}

This portion of the study involved a parametric sensitivity analysis using the previously validated computer simulation model of a manual wheelchair occupied by a mid-sized anthropomorphic test device (ATD) undergoing an LATV emergency braking/left-turn maneuver. To goal was to describe the sensitivity of key outcome measures to chosen parameters in an LATV emergency braking maneuver. Parameters varied (independent variables) in this study included the rear tiedown securement point height, wheelchair center of gravity (CG) height, increased wheelchair mass, removal of wheelchair footrests, number of tiedowns, occupant restraint system (ORS) disuse, ORS misuse, increased ATD mass, increased pulse severity, and varying LATV suspension stiffness. The outcome measures (dependent variables) monitored as means of quantifying wheelchair and wheelchair passenger instability were peak rear tiedown loading, peak fore/aft and lateral wheelchair excursions, peak fore/aft and lateral 
wheelchair tilt, and peak fore/aft and lateral ATD excursions. Findings revealed that when the ORS was not employed the ATD will be ejected during an LATV emergency braking maneuver. Increased wheelchair mass yielded the highest rear tiedown loading, while use of the ORS without employment of the tiedowns produced the highest wheelchair fore/aft excursions. Lateral wheelchair excursions were substantially less than fore/aft wheelchair excursions. Fore/aft and lateral wheelchair tilt was negligible for all parameter variation, while ATD fore/aft and lateral excursions only marginally exceeded the model baseline values.

\section{Introduction}

Although large accessible transit vehicles (LATVs) are considered a safe mode of transportation by most with their low fatality incidence of all passengers ${ }^{1}$, studies show that LATVs may not be as safe for passengers who remain seated in their wheelchairs during transit $3,4,6,10$. While injuries to the general ridership are uncommon in LATVs, normal and emergency driving maneuvers have been reported to cause wheelchair passenger injuries ${ }^{3,7}$. Proper usage of wheelchair tiedown and occupant restraint systems (WTORS) would certainly be expected to provide safe transit under normal LATV

driving conditions. However, WTORS disuse and misuse has been documented ${ }^{3,21}$, in part because the 4-pont, belt type WTORS are cumbersome to use and do not allow for wheelchair passenger independence with regard to wheelchair securement and passenger restraint.

We have examined the consequences of WTORS disuse and misuse experimentally in previous chapters for normal and emergency LATV driving. However, 
these investigations have been limited to fixed parameters: one LATV make and model, one manual wheelchair model, one surrogate wheelchair passenger, and limited WTORS configurations. In general, experimental testing described in previous chapters is constrained because of its comprehensive and economic limitations. Specifically, we were not able to gather data related to wheelchair passenger stability for varying wheelchair type, wheelchair passenger size, WTORS configuration, etc. Also, as mentioned in previous chapters, limited economic resources lead to limited LATV and LATV operator time to conduct experiments. Typically, in a parametric sensitivity analysis, various model parameters are varied and outcome measures of interest are monitored. This analysis can help us understand to which parameters our model is most sensitive. Our experimental study (chapter 4) produced data that was used to validate a computer simulation model of an adult manual transit wheelchair with a seated occupant in an LATV under emergency braking conditions. A sensitivity analysis with this validated computer model can give insight into how additional parameters related to the LATV, wheelchair, WTORS, and wheelchair passenger affect wheelchair instability during LATV emergency braking.

\section{Methodology}

Using the validated computer simulation model, a parametric sensitivity analysis was conducted which investigated the influence of various parameters on anthropomorphic test device (ATD) and wheelchair excursions, and tiedown loading. Each parameter was varied incrementally, and independently, while others were kept 
constant. Table 1 shows a summary of the model parameters (independent variables)

varied during the sensitivity analysis.

\begin{tabular}{|c|c|c|}
\hline \multirow{4}{*}{$\begin{array}{l}\text { Model } \\
\text { parameters } \\
\text { varied } \\
\text { (Independent } \\
\text { variables) }\end{array}$} & Wheelchair & $\begin{array}{l}\text { Rear tiedown securement point height } \\
\text { o Lowest point on wheelchair frame (10.2 in above ground, model } \\
\text { baseline) } \\
\text { o Mid-point on wheelchair frame (14.2 in above ground) } \\
\text { o Highest point on wheelchair frame ( } 18.1 \text { in above ground) } \\
\text { Location of the CG (vertical) } \\
\text { o Low level ( } 7.5 \text { in above ground, scooter) } \\
\text { o Mid-level ( } 11.6 \text { in above ground, power base) } \\
\text { o High level (15.4 in above ground, manual, model baseline) } \\
\text { Increased wheelchair mass } \\
\text { o Highest published mean WC mass (power bases, } 222 \mathrm{lb)} \\
\text { Removal of foot rests }\end{array}$ \\
\hline & WTORS & $\begin{array}{l}\text { Number of tiedowns used } \\
\text { o } 0 \text { (with ORS) } \\
\text { o } 0 \text { (no ORS) } \\
\text { o } 1 \text { rear, aisle-side (RLTD, no ORS) } \\
\text { o } 1 \text { front, aisle-side (FLTD, no ORS) } \\
\text { o } 2 \text { rear, (RLTD and RRTD, no ORS) } \\
\text { o } 2 \text { aisle-side, (FLTD and RLTD, no ORS) } \\
\text { Occupant restraint disuse } \\
\text { o no ORS (4 tiedowns) } \\
\text { Occupant restraint misuse } \\
\text { o LB routed around seatback (zero tiedowns) }\end{array}$ \\
\hline & ATD & $\begin{array}{l}\text { Increased ATD mass } \\
\text { o ATD mass increase from } 50^{\text {th }} \text { to } 95^{\text {th }} \text { percentile male ( } 172 \mathrm{lb} \text { to } \\
223 \mathrm{lb},+29.4 \% \text {, distributed evenly) }\end{array}$ \\
\hline & Other & $\begin{array}{l}\text { Pulse severity } \\
\text { o Bus crash pulse ( } 2 \mathrm{~g} \text {, LATV frontal crash with sedan automobile) } \\
\text { Suspension stiffness } \\
\text { o increase }+18 \% \text { suspension stiffness } \\
\text { o decrease }-18 \% \text { suspension stiffness } \\
\end{array}$ \\
\hline & ight Tiedown & $\begin{array}{l}\text { FLTD - Front Left Tiedown } \\
\text { RLTD - Rear Left Tiedown }\end{array}$ \\
\hline
\end{tabular}

Table 1. Parameters varied during sensitivity analysis with validated computer simulation model.

The securement point height was varied based on the geometric wheelchair frame constraints of the ultra-light, $x$-braced, manual wheelchair used in the model. The model baseline rear securement point height was the lowest point on the wheelchair frame $(10.2$ in above ground), and mid-point (14.2 in above ground) and highest point (18.1 in above ground) on the wheelchair frame were used as increments for variation. 
The vertical location (height above ground) of the wheelchair center of gravity (CG) was varied in the parametric sensitivity analysis based on published wheelchair CG height data ${ }^{16}$. The model baseline wheelchair CG height (15.4 in from ground) was at the upper limit of the published data. The increments chosen to vary the wheelchair CG height were the lower limit (7.5 in from ground) and mid-level (11.6 in from ground) of published values, representative of scooter and power base wheelchair CG heights, respectively.

The wheelchair mass was increased to the highest published mass of $222 \mathrm{lb}$ (mean mass of power base wheelchairs). The wheelchair footrests were also removed in the parametric sensitivity analysis as previous experience has shown that some passengers' wheelchairs are not equipped with footrests, perhaps to allow for the clearance necessary to propel the wheelchair by foot.

The number and combination of tiedowns used in the parametric sensitivity analysis was based on previous experience (chapter 2, retrospective video review of wheelchair passenger travel on LATVs): when the LATV operator uses the tiedowns to secure the wheelchair, typically only two or fewer tiedowns are employed, most often on the rear- and aisle-side. Retrospective video review of wheelchair passenger transit on LATVs also revealed that ORS disuse and misuse was very common (77.2\% and $38-72 \%$ respectively, chapter 2). ORS disuse was modeled with the absence of the lap and shoulder belt, while ORS misuse (lap belt routed around seatback) was modeled by connecting the lap belt directly to the seatback body in the model. A larger wheelchair passenger was modeled by increasing the $50^{\text {th }}$ percentile male Hybrid II ATD (172 lb, $78.2 \mathrm{~kg}$ ) mass to that of a $95^{\text {th }}$ percentile male Hybrid II ATD $(223 \mathrm{lb}, 101.2 \mathrm{~kg})$. This 
mass increase was distributed equally among the segments of the ATD in the computer model.

The crash pulse in the model was increased to a worst-case scenario: sedan automobile full frontal crash into a stationary LATV $(2 \mathrm{~g}){ }^{49}$. Figure 1 shows the filtered crash pulse used in the parametric sensitivity analysis.

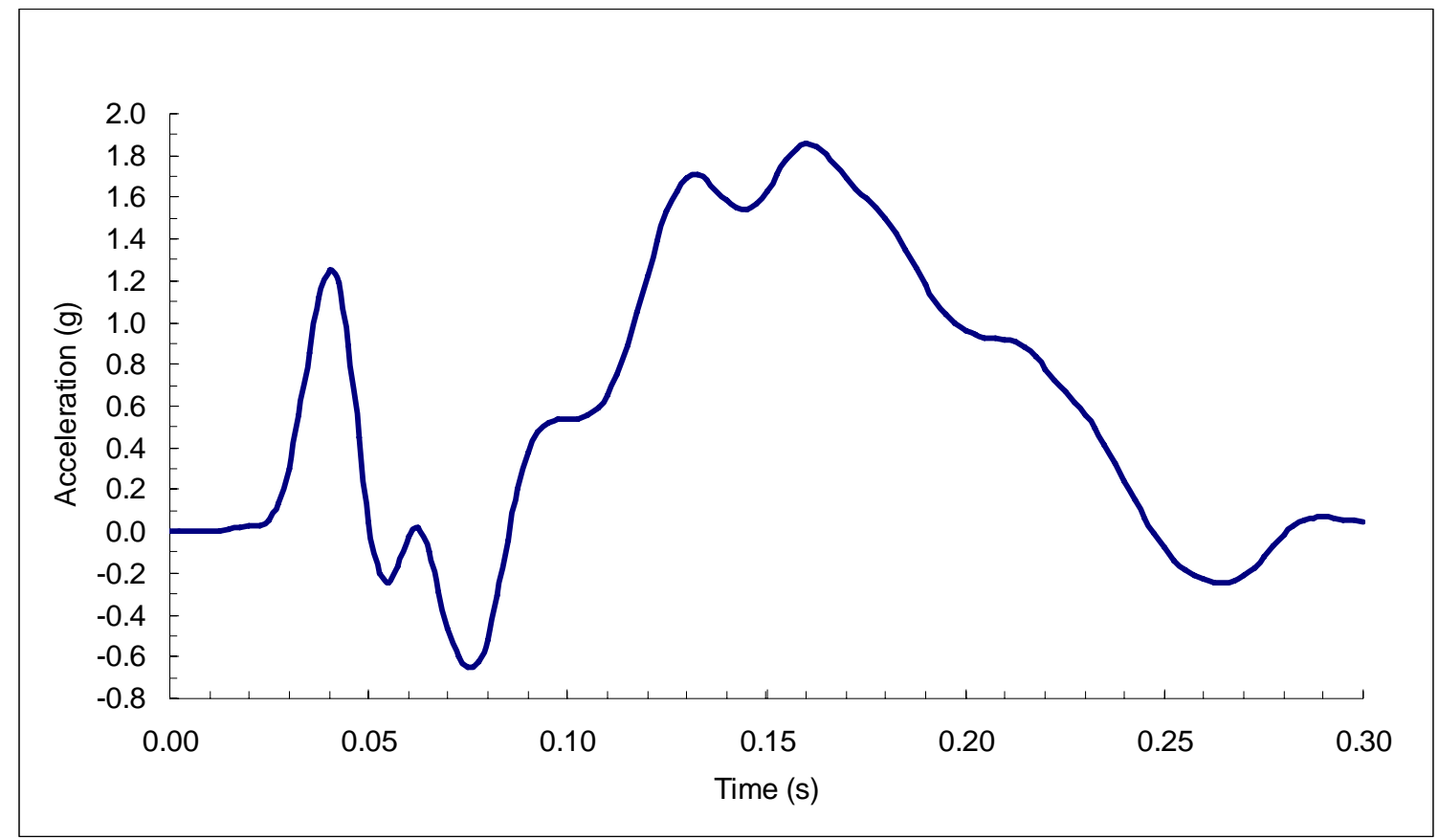

Figure 1. LATV deceleration pulse of sedan automobile frontal impact into LATV. Pulse peak is $2 \mathrm{~g}$ (frontal crash).

LATV suspension stiffness was varied $\pm 18 \%$ based on data from a study ${ }^{50}$ that showed the largest difference in vertical accelerations experienced by passengers between the LATV front and rear. The LATV suspension was modeled using a revolute joint under the securement station allowing for securement station platform fore/aft tilting. Figure 2 shows the LATV suspension stiffness range and the model baseline. 


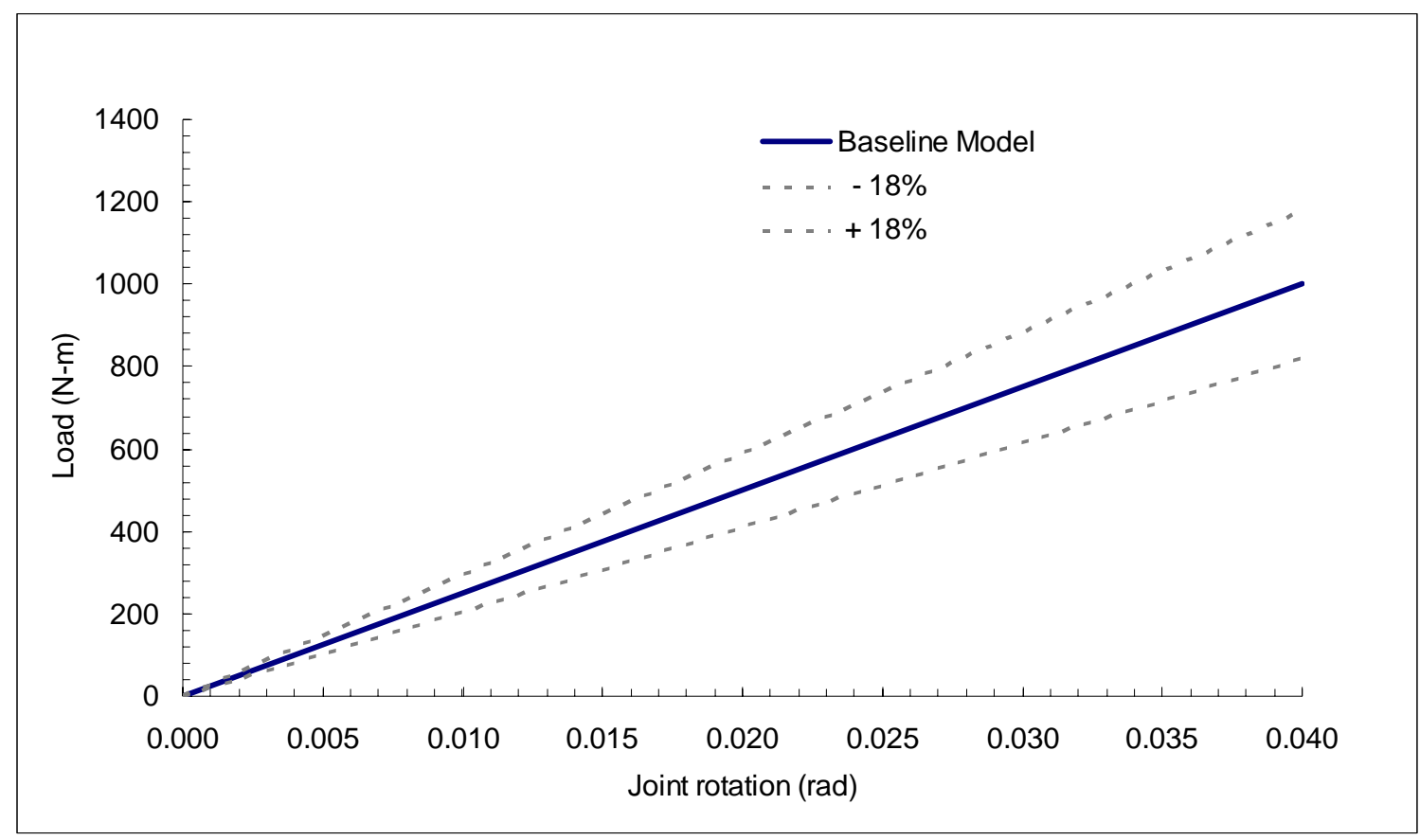

Figure 2. LATV suspension stiffness range in parametric sensitivity analysis. Computer model simulated LATV suspension during an emergency braking maneuver as a rotational joint positioned at the center of the securement station platform (allowing platform fore-aft tilt). Joint baseline stiffness in model is depicted with a solid line. The

LATV suspension stiffness was varied $\pm 18 \%$ (dashed lines) based on published acceleration differences associated with varying LATV suspensions.

Table 2 shows a list of the outcome measures (dependent variables) recorded during the parametric sensitivity analysis. These outcome measures were chosen as they were considered good indicators for wheelchair and wheelchair passenger instability and wheelchair loading.

\begin{tabular}{|l|l|l|}
\hline \multirow{4}{*}{$\begin{array}{l}\text { Outcome } \\
\text { measures } \\
\begin{array}{l}\text { (Dependent } \\
\text { variables) }\end{array}\end{array}$} & WTORS & $\begin{array}{l}\text { Peak RRTD loading } \\
\text { Peak RLTD loading }\end{array}$ \\
\cline { 2 - 3 } & Wheelchair & $\begin{array}{l}\text { Peak WC fore-aft excursion } \\
\text { Peak WC lateral excursion } \\
\text { Peak WC fore-aft tilt } \\
\text { Peak WC lateral-aft tilt }\end{array}$ \\
\cline { 2 - 3 } & ATD & $\begin{array}{l}\text { Peak ATD fore-aft excursion (at shoulder) } \\
\text { Peak ATD lateral excursion (trunk) }\end{array}$ \\
\hline
\end{tabular}

Table 2. Outcome measures monitored during sensitivity analysis. 
Sensitivity for each continuous variable parameter varied compared to baseline was reported as the sensitivity index (SI) or the ratio of the percent change in outcome measure to the percent change in input parameter.

\section{Results}

For the scenario of a manual wheelchair, occupied by an ATD, was subjected to an LATV emergency braking maneuver, we evaluated the range of tiedown loading, and wheelchair and wheelchair passenger excursions for varying parameters. Furthermore, this study identified outcome measure sensitivity to parameter variation. The results from the parametric sensitivity analysis are summarized in Table 3. 


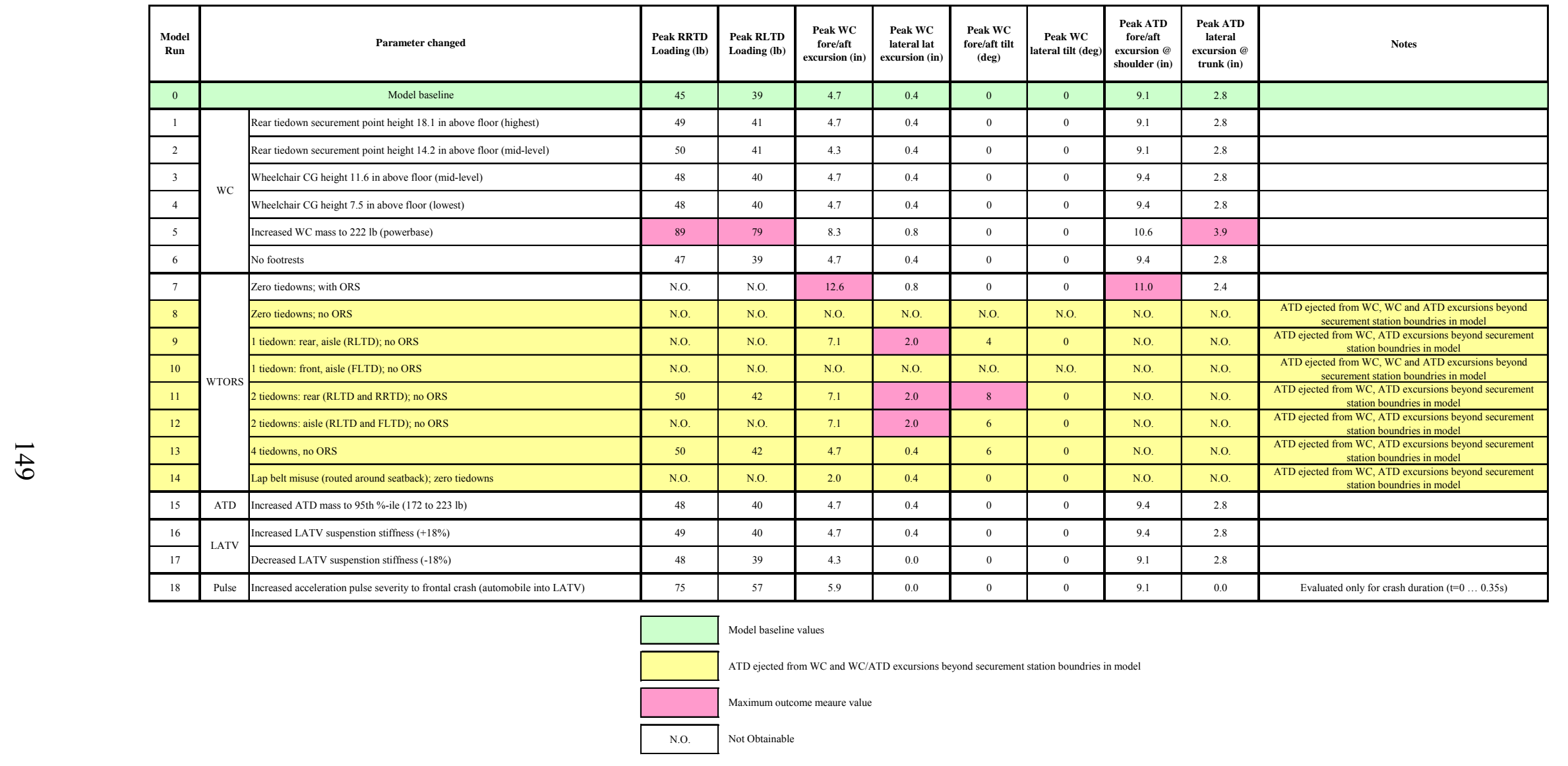

Table 3. Parametric sensitivity analysis results with peak outcome measure values. Green shading indicates model baseline values, red shading indicates overall maximum outcome measure values, and yellow shading indicates either ATD ejection from wheelchair or wheelchair/ATD excursions beyond the securement station boundaries in the model. N.O. indicates outcome measure values that were not obtainable for that particular run (e.g. ATD ejection could not yield relevant ATD excursions, rear tiedown loading could not be reported when zero rear tiedowns were used, etc.). 
The tiedown loading ranged from 39 to $79 \mathrm{lb}$ for the RLTD and from 45 to $89 \mathrm{lb}$ for the RRTD, with highest overall peak tiedown loading for both rear tiedowns occurring with wheelchair mass increase (222 lb to represent power base wheelchair). The highest overall peak wheelchair fore/aft excursion of 12.6 in (7.9 in beyond the model baseline) occurred when no tiedowns were used to secure the wheelchair and the ORS was employed. Highest overall peak wheelchair lateral excursion of 2.0 in (1.6 in beyond the model baseline) occurred during 1-tiedown (RLTD only), and both 2-tiedown (rear- and aisle-side) WTORS configurations. The highest overall peak wheelchair fore/aft tilt was $8^{\circ}$, while there was no wheelchair lateral tilt found throughout the parametric sensitivity analysis. The highest overall peak ATD fore/aft excursion (when ATD was not ejected) was 11.0 in (1.9 in beyond the model baseline), occurring when no tiedowns were used and the ORS was employed. The highest overall peak ATD lateral excursion was 3.9 in (1.1 in beyond the model baseline), occurring when the wheelchair mass was increased to $222 \mathrm{lb}$. In runs (8-14) when the ORS were either disused or misused (the ATD was not restrained properly), the ATD was ejected from the wheelchair.

Figures 3-10 graphically depict the range of outcome measure values caused by each continuous variable parameter change. 


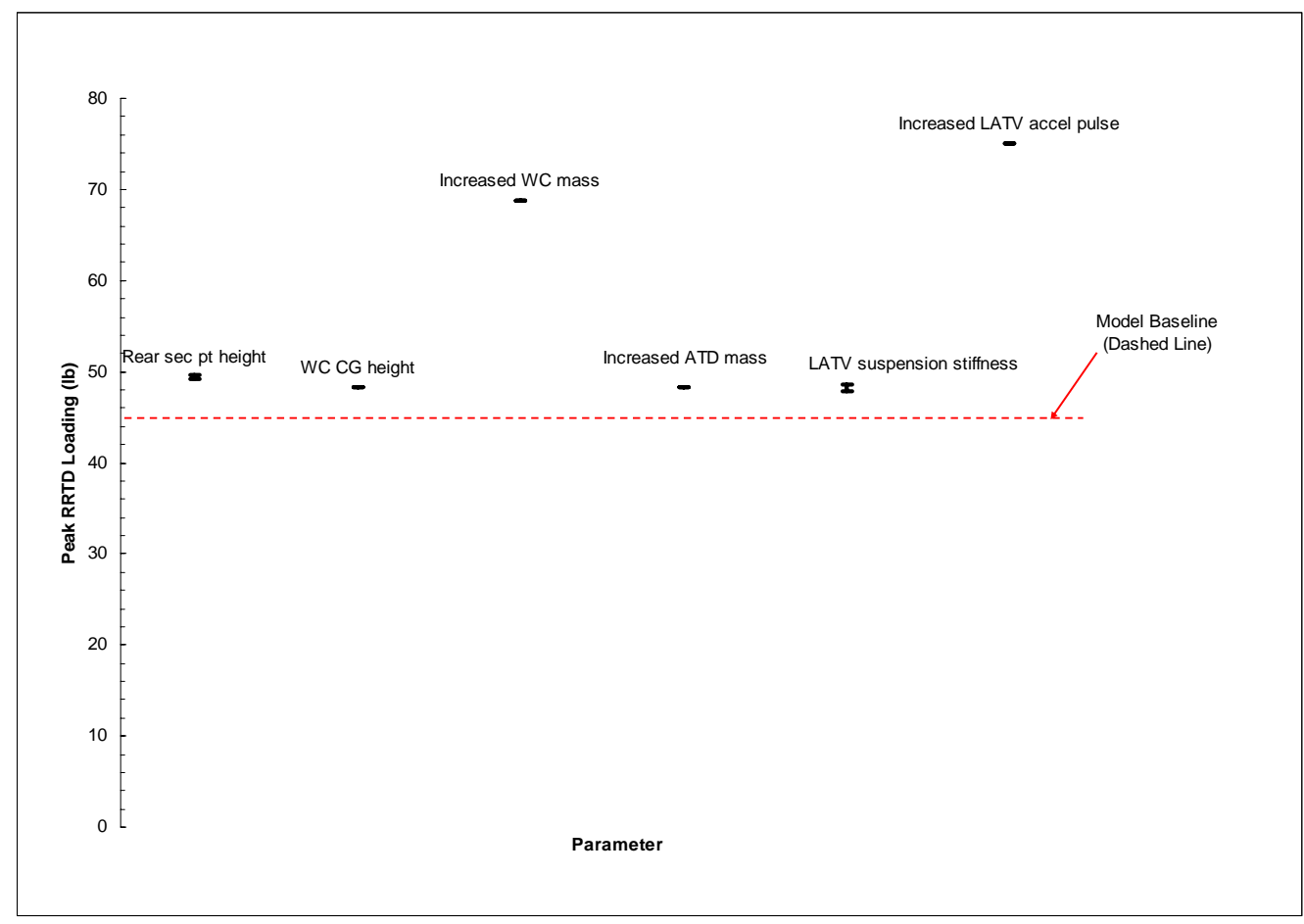

Figure 3. Range of peak RRTD loading values for each parameter varied in the sensitivity analysis. Red dashed line shows model baseline value.

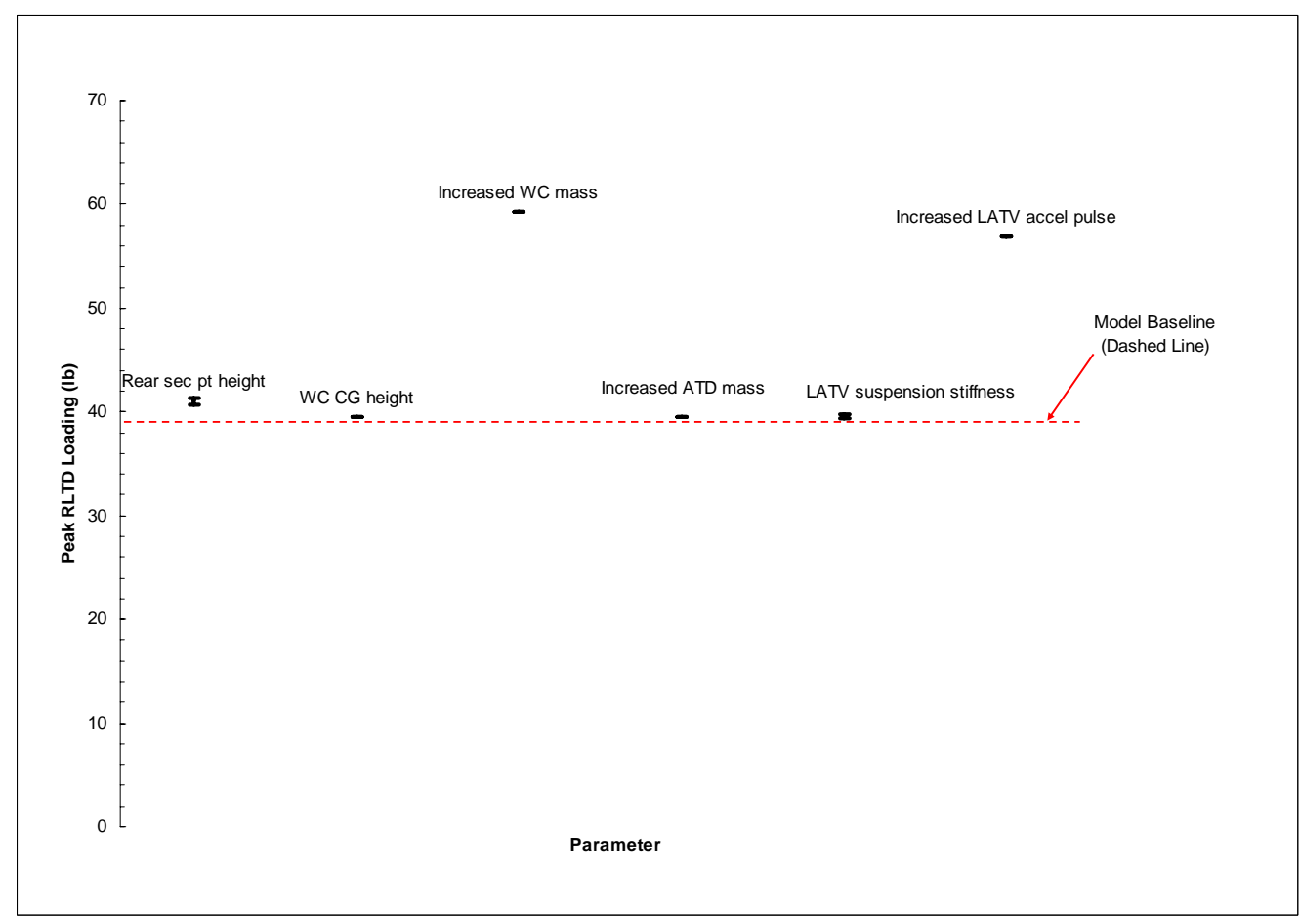

Figure 4. Range of peak RLTD loading values for each parameter varied in the sensitivity analysis. Red dashed line shows model baseline value. 


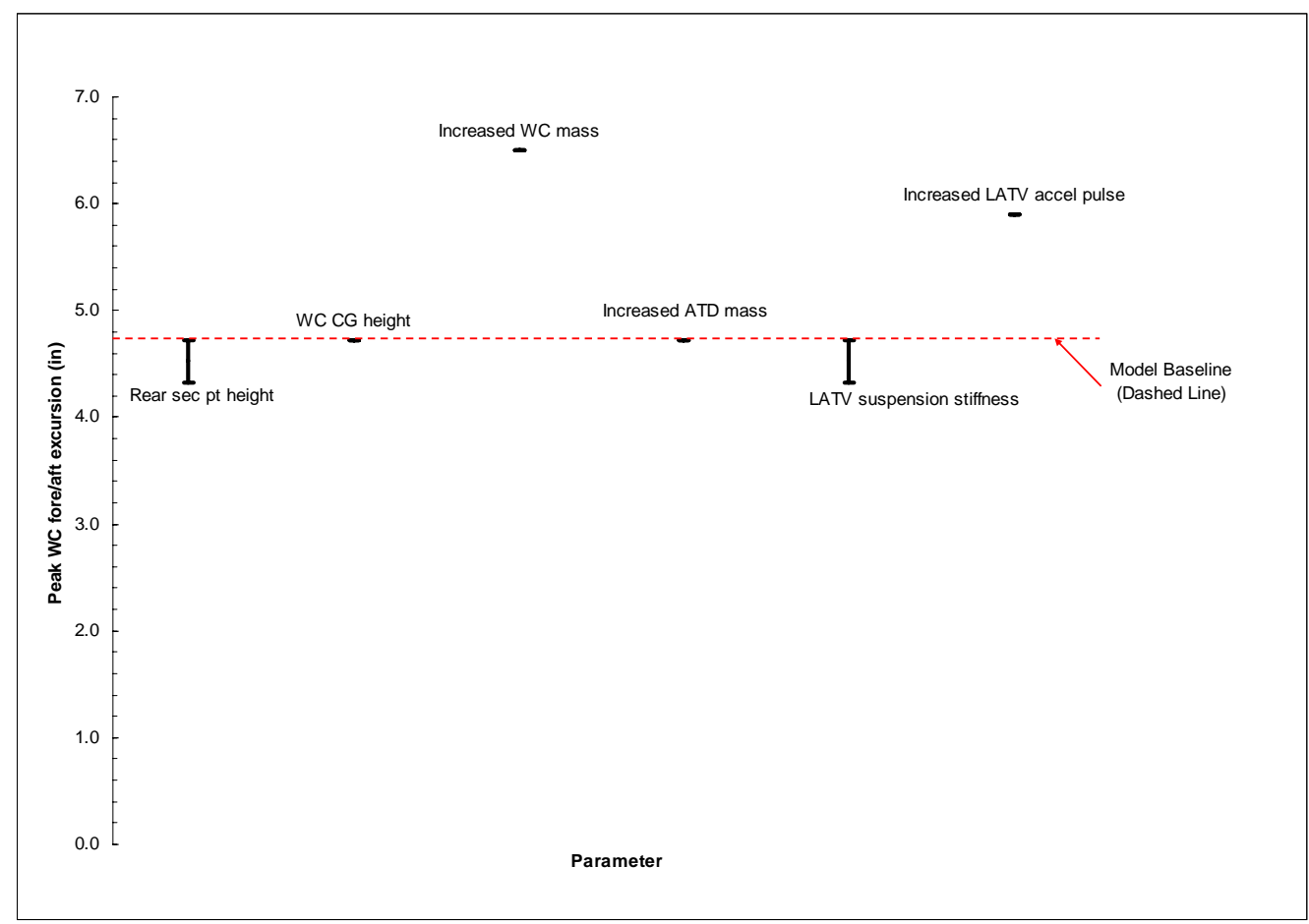

Figure 5. Range of peak wheelchair fore/aft excursion values for each parameter varied in the sensitivity analysis. Red dashed line shows model baseline value.

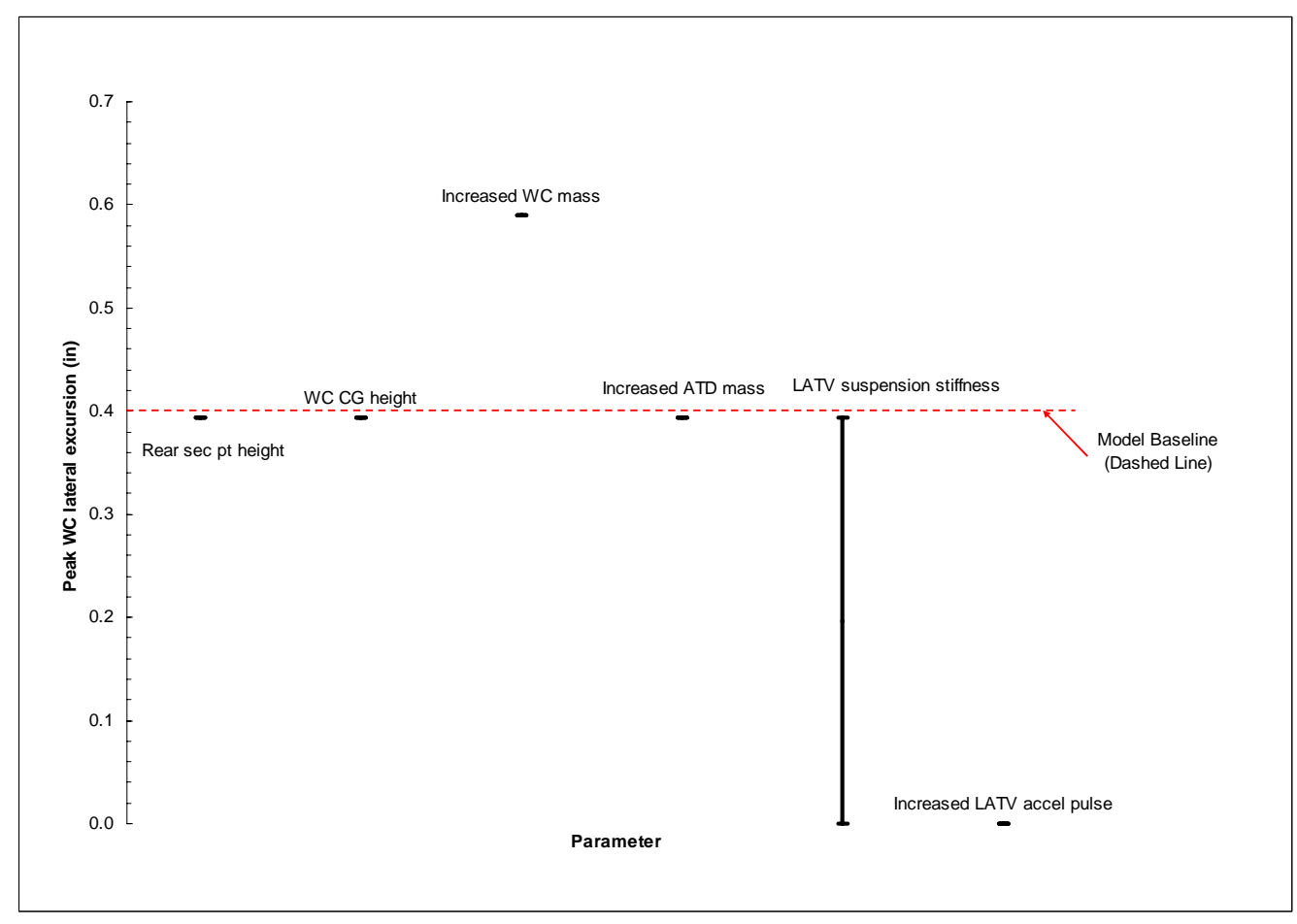

Figure 6. Range of peak wheelchair lateral excursion values for each parameter varied in the sensitivity analysis. Red dashed line shows model baseline value. 


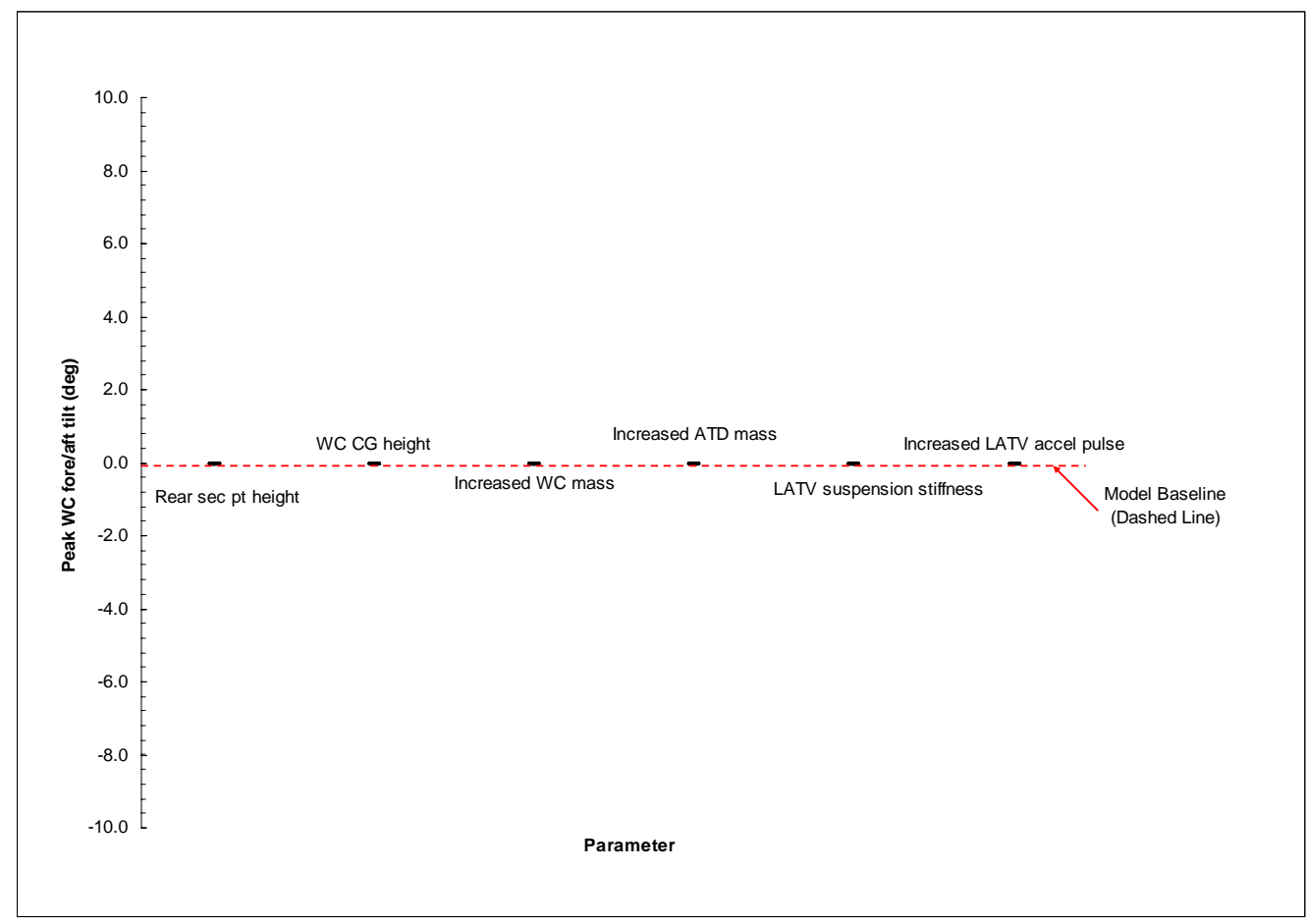

Figure 7. Range of peak wheelchair fore/aft tilt values for each parameter varied in the sensitivity analysis. Red dashed line shows model baseline value.

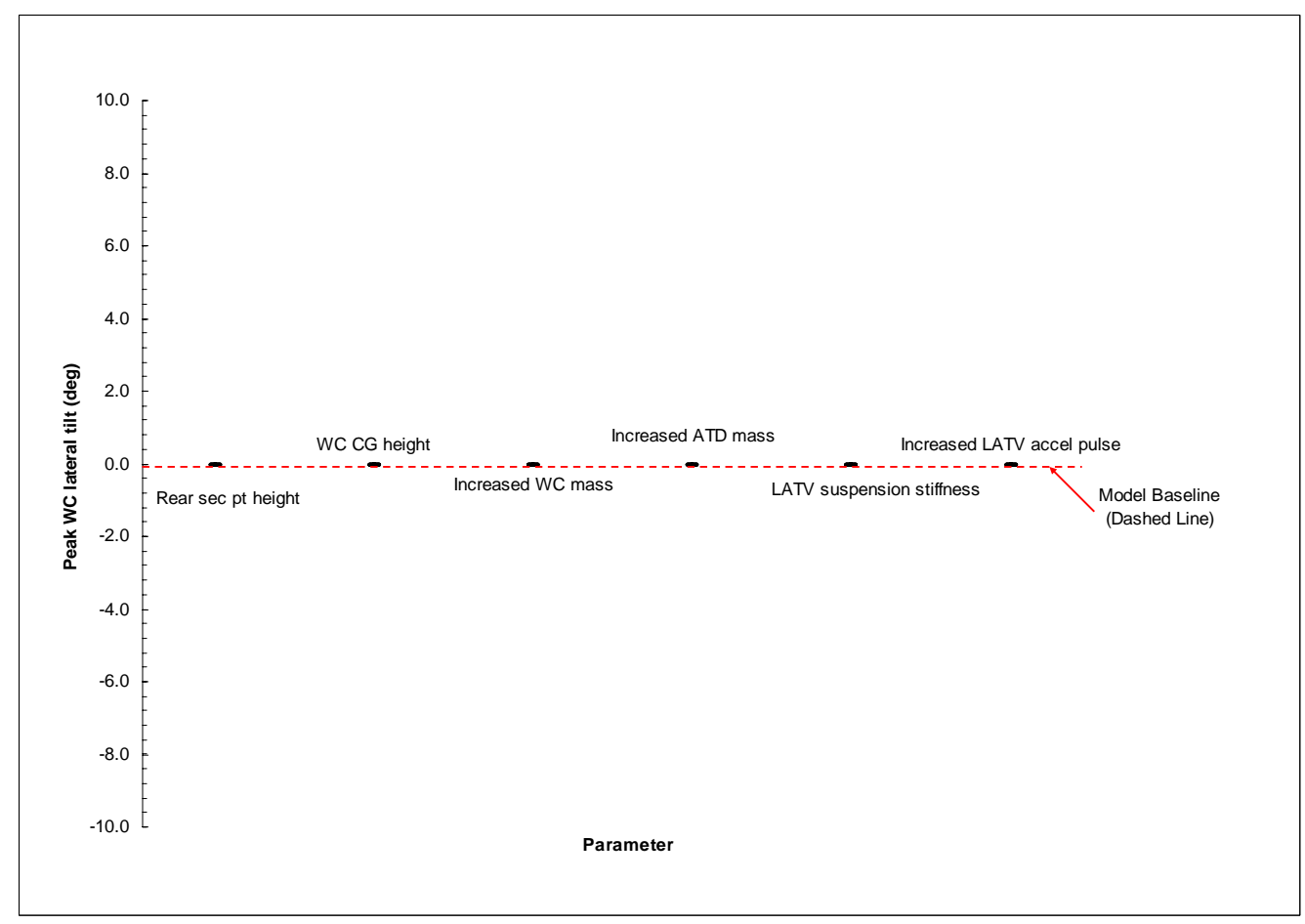

Figure 8. Range of peak wheelchair lateral tilt values for each parameter varied in the sensitivity analysis. Red dashed line shows model baseline value. 


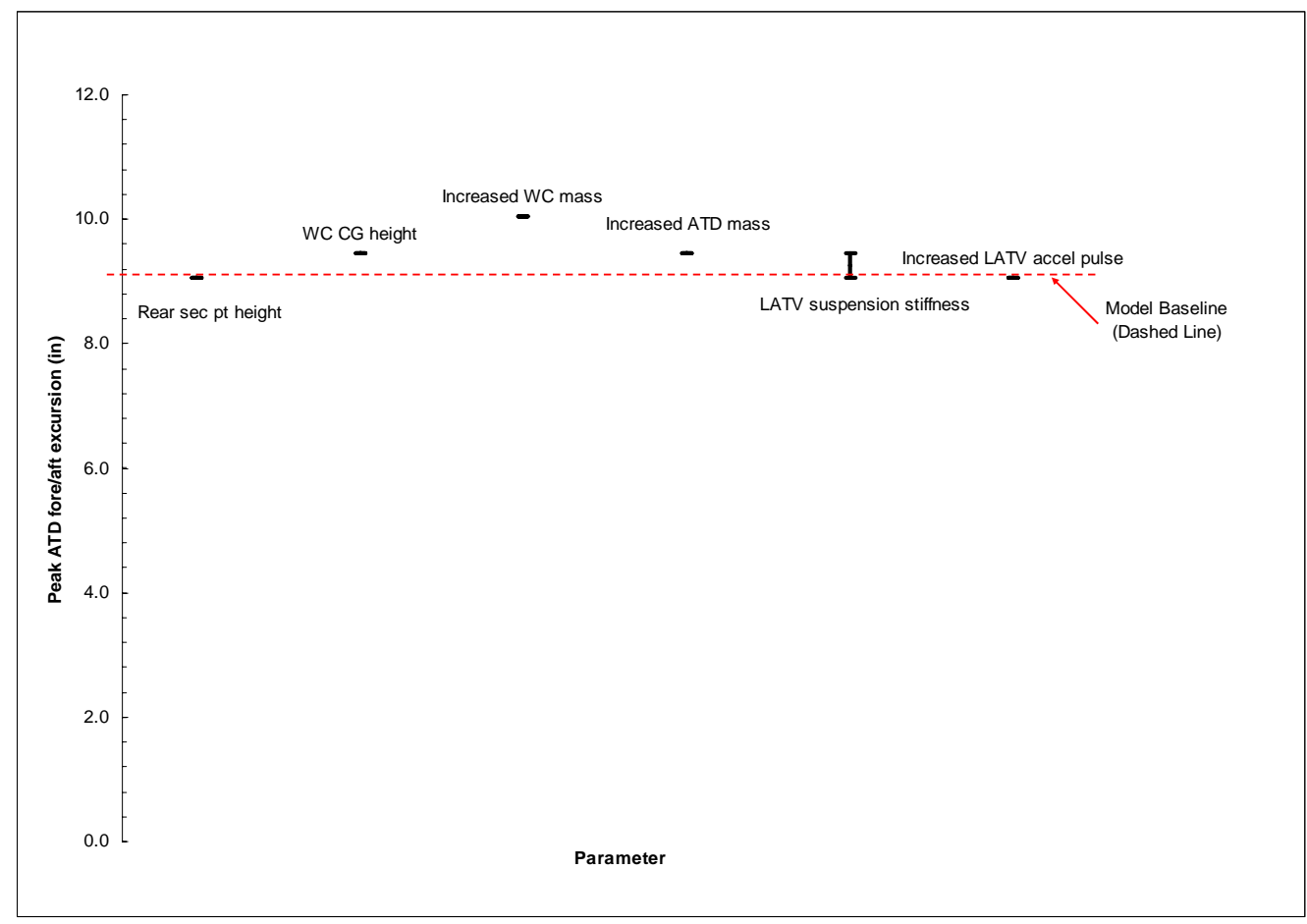

Figure 9. Range of peak ATD fore/aft excursion values for each parameter varied in the sensitivity analysis. Red dashed line shows model baseline value.

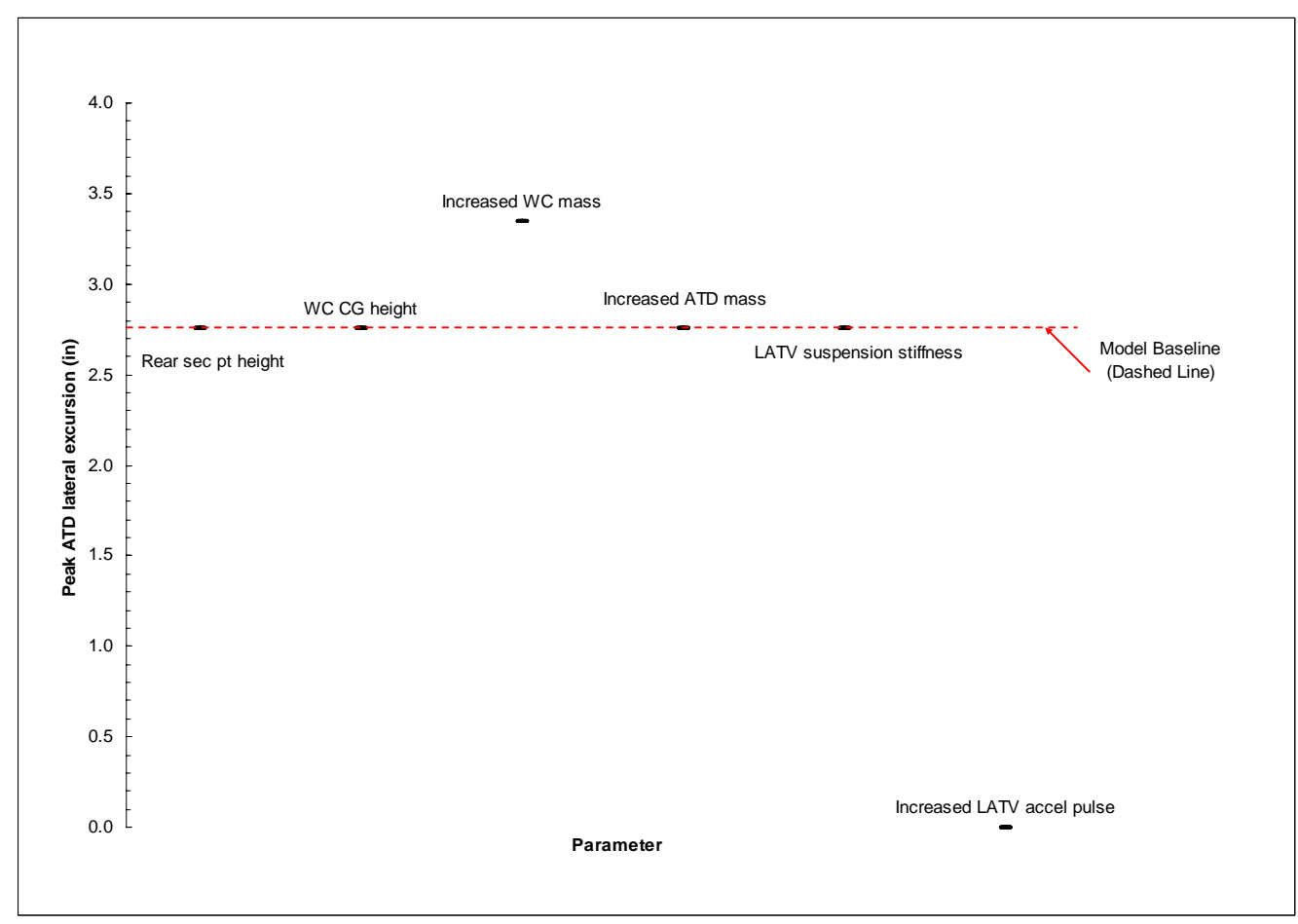

Figure 10. Range of peak ATD lateral excursion values for each parameter varied in the sensitivity analysis. Red dashed line shows model baseline value. 
The SI values for continuous variable parameters are shown in Table 4. Peak rear tiedown loading was most sensitive to the increased LATV acceleration pulse, while peak wheelchair excursions were most sensitive to decreased LATV suspension stiffness. Wheelchair fore/aft and lateral tilt was not sensitive to any parameter variation. The peak ATD fore/aft excursions were most sensitive to increased LATV suspension stiffness, while peak ATD lateral excursions were most sensitive to the increased LATV acceleration pulse. 


\begin{tabular}{|c|c|c|c|c|c|c|c|c|c|c|}
\hline $\begin{array}{l}\text { Model } \\
\text { Run }\end{array}$ & & Parameter changed & $\begin{array}{l}\text { Peak RRTD } \\
\text { Loading }\end{array}$ & $\begin{array}{c}\text { Peak RLTD } \\
\text { Loading }\end{array}$ & $\begin{array}{c}\text { Peak WC } \\
\text { fore/aft } \\
\text { excursion }\end{array}$ & $\begin{array}{l}\text { Peak WC } \\
\text { lateral lat } \\
\text { excursion }\end{array}$ & $\begin{array}{c}\text { Peak WC } \\
\text { fore/aft tilt }\end{array}$ & $\begin{array}{l}\text { Peak WC } \\
\text { lateral tilt }\end{array}$ & $\begin{array}{c}\text { Peak ATD } \\
\text { fore/aft } \\
\text { excursion @ } \\
\text { shoulder }\end{array}$ & $\begin{array}{c}\text { Peak ATD } \\
\text { lateral } \\
\text { excursion @ } \\
\text { trunk }\end{array}$ \\
\hline 1 & \multirow{5}{*}{ WC } & Rear tiedown securement point height 18.1 in from floor (highest) & 0.11 & 0.07 & 0.00 & 0.00 & 0.00 & 0.00 & 0.00 & 0.00 \\
\hline 2 & & Rear tiedown securement point height 14.2 in from floor (mid-level) & 0.24 & 0.09 & 0.21 & 0.00 & 0.00 & 0.00 & 0.00 & 0.00 \\
\hline 3 & & Wheelchair CG height 11.6 in from floor (mid-level) & 0.25 & 0.02 & 0.00 & 0.00 & 0.00 & 0.00 & 0.17 & 0.00 \\
\hline 4 & & Wheelchair CG height 7.5 in from floor (lowest) & 0.12 & 0.01 & 0.00 & 0.00 & 0.00 & 0.00 & 0.08 & 0.00 \\
\hline 5 & & Increased WC mass to $222 \mathrm{lb}$ (powerbase) & 0.18 & 0.19 & 0.14 & 0.19 & 0.00 & 0.00 & 0.03 & 0.08 \\
\hline 15 & ATD & Increased ATD mass to 95 th $\%$-ile (172 to $223 \mathrm{lb}$ ) & 0.22 & 0.02 & 0.00 & 0.00 & 0.00 & 0.00 & 0.15 & 0.00 \\
\hline 16 & \multirow{2}{*}{ LATV } & Increased LATV suspenstion stiffness (+18\%) & 0.39 & 0.06 & 0.00 & 0.00 & 0.00 & 0.00 & 0.24 & 0.00 \\
\hline 17 & & Decreased LATV suspenstion stiffness $(-18 \%)$ & 0.30 & 0.00 & 0.46 & 5.56 & 0.00 & 0.00 & 0.00 & 0.00 \\
\hline 18 & Pulse & Increased acceleration pulse severity to frontal crash (automobile into LATV) & 0.44 & 0.30 & 0.17 & 0.67 & 0.00 & 0.00 & 0.00 & 0.67 \\
\hline
\end{tabular}

\begin{tabular}{ll}
\hline \\
\hline
\end{tabular} Maximum Sensitivity Index

Table 4. Sensitivity indices (SI) for continuous variable parameters. SI is the ratio of the percent change in outcome measure to the percent change in input parameter. The highest SI for each outcome measure is indicated by a shaded red box. 


\section{Discussion and Conclusions}

An important finding in our parametric sensitivity analysis is that the ATD was ejected in all cases when no ORS were used to restrain the wheelchair passenger (runs 814). Our previous study (chapter 2$)$ showed a high rate of ORS misuse and disuse (49.1\% and $26.3 \%$ respectfully). These results suggest that wheelchair passenger injury risk may increase substantially if the ORS is not used to restrain the wheelchair passenger as the LATV undergoes an emergency braking maneuver. This was also substantiated in the experimental portion of our study where the ATD was ejected from the wheelchair as the LATV underwent various emergency braking and emergency braking/left-turn maneuvers. It should be noted that in parametric sensitivity analysis runs when the ATD was ejected or wheelchair/ATD excursions were beyond the securement station boundaries in the model, we were not able to report all outcome measure values (N.O., not obtainable, Table 3). The subsequent discussion does not include data from these simulations.

As anticipated, the peak rear tiedown loading across all parametric variation in this study was lower than rear tiedown loading reported in other studies ${ }^{30-34}$ evaluating high-g deceleration environments. In our study, the highest overall peak RRTD and RLTD loading was 89 and $79 \mathrm{lb}$ respectively. In previous studies simulating high-g frontal impacts ${ }^{30,32,33}$, the peak rear tiedown load (per tiedown) ranged from 1400.1$4728.4 \mathrm{lb}$. Rear impact studies ${ }^{31,34}$ reported peak rear tiedown loads per tiedown ranging from 77.6-427.1 lb. As expected, tiedown loads in our parametric analysis of LATV emergency braking are substantially less than those during simulated impact events, with the exception of the $77.6 \mathrm{lb}$ in the Fuhrman study ${ }^{12}$ that involved a pediatric wheelchair 
passenger seated in a pediatric wheelchair. Since most WTORS are designed for high-g (crash) environments, they may be "overly robust" for LATV normal driving conditions. However, the rear tiedown loading range in this study (RRTD 45-89 lb, and RLTD 39-79 lb) was substantially higher than the rear tiedown loading in LATV normal braking maneuvers (mean peak: RRTD $9.0 \mathrm{lb}$, and RLTD $6.5 \mathrm{lb}$ ). This increase in rear tiedown loading can be attributed to the higher resultant LATV deceleration associated with the emergency braking maneuver compared to normal braking maneuvers $(0.8 \mathrm{~g}$ vs. $0.2 \mathrm{~g}$ respectively). The LATV deceleration pulse in this study also included a lateral component, as the LATV was also turning left while braking, which led to a further increase in LATV resultant deceleration compared to normal LATV braking. The parametric sensitivity analysis revealed that the highest rear tiedown loading occurred when wheelchair mass was increased. This is not surprising, since with increased mass, a wheelchair has greater momentum as it tends to move forward during an LATV emergency braking maneuver, ultimately leading to increased rear tiedown loading.

Excluding run 7, wheelchair fore/aft excursions ranged from model baseline of 4.5 in to 8.3 in (when wheelchair mass was increased from $35 \mathrm{lb}$ to $222 \mathrm{lb}$ to represent a power base wheelchair). In run 7, when no tiedowns were used and only the ORS was employed, the highest measured peak wheelchair fore/aft excursion of 12.6 in occurred. This relatively high wheelchair fore/aft excursion occurred because no tiedowns were in use to keep the wheelchair secured during LATV deceleration (the ORS not only restrained the ATD in this instance, but also served to "restrain" the wheelchair). The wheelchair and ATD dynamics during a braking maneuver allowed for the ORS to serve as a de facto wheelchair "restraint," since the wheelchair and ATD rolled forward during 
LATV deceleration. In LATV acceleration, when the wheelchair and ATD tend to move rearward, the ORS would not be able to "restrain" the wheelchair. Additionally, when relying on the ORS to "restrain" a heavier power base wheelchair during a higher-g deceleration pulse, the wheelchair passenger may experience substantially higher shoulder and lap belt loads. In this case, the occupant and wheelchair have greater momentum as they move forward and the ORS is loaded, resulting in substantially higher shoulder and lap belt loading. Therefore, a WTORS configuration that does not feature the use of tiedowns may put the wheelchair passenger at increased risk of injury. This is the reason the tiedowns and occupant restraints were designed to work together to keep the wheelchair passenger safe during transit.

Lateral wheelchair excursions (0.4-2.0 in) were lower compared to fore/aft wheelchair excursions (4.5-12.6 in) for all varied parameters. This is because the major component of LATV deceleration pulse in the model is in the fore/aft direction $(0.8 \mathrm{~g})$ with a smaller lateral component $(0.3 \mathrm{~g})$. Furthermore, the folded-seat barrier at the right side of the securement station may limit tendency for lateral wheelchair excursion. During an LATV maneuver that includes LATV turning left (such as the one in this study) the wheelchair tends to continue moving to the right, causing contact with the folded-seat barrier at the right side if the securement station.

Throughout the parametric sensitivity analysis, wheelchair fore/aft and lateral tilt was relatively negligible and only occurred as a result of ATD ejection from the wheelchair. Highest overall peak ATD fore/aft excursions of 11.0 in, obtained when the ATD was not ejected, were only marginally higher than the model baseline $9.1 \mathrm{in}$. ATD 
lateral excursions increased only marginally above model baseline values, as well, evidenced by the highest overall peak of 3.9 in compared to model baseline of $2.8 \mathrm{in}$.

Table 4 shows the continuous variable parameters to which the model's outcome measures are most sensitive. The SI show that the model is most sensitive to increased LATV acceleration pulse (during a frontal impact with a sedan automobile) and varying LATV suspension stiffness. Even though the model is most sensitive to these two parameters, review of the outcome measure values in Table 3 suggest that the two parameters may be within a range that is not likely to cause adverse events. This is especially true when considering the outcome associated with variations in noncontinuous variables (WTORS configurations).

\section{$\underline{\text { Limitations }}$}

This parametric sensitivity analysis was performed using a validated computer simulation model of a manual wheelchair passenger subjected to an LATV emergency braking/left-turn maneuver. The findings in this study may be different under different LATV emergency maneuvers, where the ATD and wheelchair dynamics differ.

The SI was only calculated for continuous variable parameters (such as height, mass, and stiffness). Variation of categorical variable parameters such as WTORS configurations and use of footrests produced important results (such as ATD ejections) that cannot be directly compared using the SI. However, the outcomes resulting from variation of categorical variable parameters may be substantial.

Rationale for varying wheelchair CG height and wheelchair mass were based on a study ${ }^{16}$ published in 1997 . While more than a decade has passed since the publication of 
these data and today's wheelchairs may have different CG height and mass, a standard literature search failed to produce more recently published studies describing these wheelchair characteristics.

The variation of LATV suspension stiffness was based on published differences in vertical acceleration along the LATV longitudinal axis ${ }^{50}$. Ideally, a range of published suspension stiffnesses would have been used in the parametric sensitivity analysis, but several attempts to obtain this information from the LATV manufacturer were unsuccessful.

In this parametric sensitivity analysis, the parameters were varied independently; varying two or more parameters together may have produced different results. The $2 \mathrm{~g}$ LATV crash pulse ${ }^{50}$ was digitized and filtered (low-pass Butterworth filter, cutoff frequency $=16 \mathrm{~Hz}$ ), before it could be used as an model input in the parametric sensitivity analysis, which may have introduced errors.

The ATD mass increase was done uniformly across all ATD segments; a wheelchair passenger of greater mass may have non-uniform mass distribution, which could alter the excursion and loading results. The wheelchair mass increase and wheelchair CG height variations were not accompanied by geometric changes to the wheelchair (e.g. different wheels, etc.). None of these changes would have been possible without extensive changes to the model, which may have reduced its validity.

ATD and wheelchair excursions were not be reported (N.O., not obtainable in Table 3) in cases when the ATD was ejected and/or the wheelchair/ATD excursions exceeded the securement station boundaries in the model. Our model did not feature the securement station front bulkhead, but wheelchair/ATD excursions beyond the 
securement station boundaries would certainly result in impact with the LATV interior. These simulations were designated separately through the use of the color yellow in Table 3 .

This study is specific to evaluating 4-point, strap-type WTORS variations. The findings from this study may not be applicable to the evaluation of alternative wheelchair securement devices, such as the auto-docking system, and the rear- and front-facing containment stations. While the computer model needs to be re-validated to investigate these alternative approaches to wheelchair securement, the findings from this study can provide general considerations for researchers and designers as they further develop these systems.

In conclusion, increased wheelchair mass yielded the highest rear tiedown loading, while use of the ORS without employment of the tiedowns produced the highest wheelchair fore/aft excursions (not including cases where the ATD was ejected or wheelchair/ATD excursions were beyond the securement station boundaries). Lateral wheelchair excursions were substantially less than fore/aft wheelchair excursions, as the tendency for lateral movement was limited by the folded seat barrier. The fore/aft and lateral wheelchair tilt were negligible for all runs, while ATD fore/aft and lateral excursions only marginally exceeded the model baseline values. This study also found when the ORS is not employed the ATD will be ejected during an LATV emergency braking maneuver. 


\section{CHAPTER 8}

\section{SUMMARY AND CONCLUSIONS}

\section{Key Findings and Recommendations}

Large accessible transit vehicles (LATVs) are an important form of transportation for wheelchair users, giving them access to employment, recreation, healthcare and other activities of daily living. Although this is generally considered a safe mode of transportation, for wheelchair users, traveling on LATVs can present an increased risk of adverse events and injury. This is particularly true with regard to the disuse and misuse of four-point, strap-type wheelchair tiedown and occupant restraint systems (WTORS), which are meant to protect wheelchair passengers during transit. The overall aim of this study was to describe and understand the consequences of WTORS disuse and misuse under LATV normal and emergency driving conditions. Furthermore, we sought to identify key factors contributing to adverse events for wheelchair occupants during transit, and to use these newly identified factors to make recommendations for promoting improved wheelchair passenger safety on LATVs. To our knowledge, this is the first study aimed at describing the potential consequences of WTORS disuse and misuse for wheelchair passengers. 
In the first portion of our study, we reviewed video footage of wheelchair passenger transit on LATVs. Our study confirmed the findings of previous studies, suggesting that WTORS are often disused and misused to secure wheelchairs and restrain wheelchair passengers. In cases of wheelchair or wheelchair passenger instability, the most common WTORS configuration was the misuse of the lap belt in an attempt to secure the wheelchair (but not to restrain the wheelchair passenger) by routing the belt around the wheelchair seat back, and no use of wheelchair tiedowns. In the adverse events identified, the vast majority involved minor instability and only a small number were severe instability cases. The three cases of severe instability suggested that wheelchair-related adverse events could occur during normal LATV driving maneuvers when WTORS are not properly employed. In addition, we found that ambulatory passengers may also be at increased risk of injury if a wheelchair related adverse event occurs during LATV transit. Based on these findings, we suggest that transit agency policies require wheelchair users to apply wheelchair brakes, turn off power wheelchairs, employ all four tiedowns correctly, and use the lap and shoulder belts. Furthermore, our findings suggest that additional training and monitoring of LATV operators with regard to WTORS use would improve not only wheelchair passenger safety, but safety for all passengers.

Next, we sought to describe accelerations and WTORS loads experienced by manual wheelchair passengers under LATV normal and emergency driving conditions. Under both normal and emergency driving conditions, WTORS loading measured in our study was substantially less than WTORS loading reported in other studies with high-g (crash) deceleration environments. When WTORS are properly used, the wheelchair 
passenger does not appear to be at increased risk of injury, given that the ATD remained seated in an upright, supported position. WTORS disuse and misuse are associated with a high frequency of wheelchair passenger ejection from the wheelchair, especially during an emergency maneuver that involves any form of LATV braking (braking, leftturn/braking, and right-turn/braking). These data may prove helpful in advancing alternative approaches to low-g wheelchair securement/containment and passenger restraint, such as the forward-facing ${ }^{22}$ and rear-facing ${ }^{17}$ wheelchair passenger stations and auto-docking systems ${ }^{18}$. Specifically, we recommend that design of alternative systems to be used on LATVs place special emphasis on the ease-of use, while being robust enough to withstand loads associated with low-g environments $(\leq 3 \mathrm{~g})$.

While studying the kinematic environment under normal driving conditions, we observed that the manual wheelchair brake mounting hardware condition can play a major role in influencing wheelchair excursions when WTORS are not properly used. We found that vibrations associated with LATV normal driving loosened the brake mounting hardware. This leads us to recommend regular brake inspections to assure that brake mounting hardware is securely clamped to the wheelchair frame for transit users. There is also a need for improved brake mounting hardware design for manual transit wheelchairs. Both can increase wheelchair passenger safety during LATV transit. We also found that wheelchair users with limited upper body and trunk control may be more susceptible to wheelchair tipping if their wheelchair brake is engaged and tiedowns are not properly used. Specifically, a relatively short wheelbase (a typical characteristic in ultra-light manual wheelchairs and needed for wheelchair maneuverability) coupled with the surrogate wheelchair passenger's inability for balancing was associated with an increased 
likelihood of tipping, even under LATV normal driving conditions. This underscores the importance of involving occupational and/or physical therapists in the process of wheelchair prescription, as prescribing a wheelchair appropriate for the user's abilities/needs may play a substantial role in their transportation safety.

We also found that the ORS retractor locking mechanisms may not engage effectively in a low-g $(<1 \mathrm{~g})$ environment. This became apparent when the wheelchair brake mounting hardware was loosely clamped to the wheelchair frame, as the wheelchair brake did not effectively prevent forward wheelchair excursions. Even though the lap belt was misused in an attempt to secure the wheelchair, it is sensible to expect that during LATV braking, the belt retractor locking mechanism would engage and prevent forward wheelchair excursions. Four-point, strap-type wheelchair tiedowns and occupant restraints were designed to keep wheelchairs secured and wheelchair occupants restrained in a high-g crash environment. Hence, WTORS retractor locking mechanisms were designed to engage in a high-g crash environment; we found they may not engage effectively in a low-g $(<1 \mathrm{~g})$ environment associated with normal LATV driving maneuvers. This leads us to recommend the design of retractors suitable for low-g $(<1 \mathrm{~g})$ environments.

Finally, we used data gathered during an emergency LATV driving maneuver (braking/left-turn) to develop and validate a computer model simulating the same maneuver as experienced by a commercial adult manual wheelchair occupied by a surrogate wheelchair passenger. This model was then used to conduct a parametric sensitivity analysis to identify additional parameters related to the LATV, wheelchair, WTORS, and wheelchair passenger that may affect wheelchair instability during LATV 
emergency braking. Parametric sensitivity analysis revealed that the model is most sensitive to the following continuous parameters: LATV acceleration pulse and LATV suspension stiffness. However, the most substantial finding from the parametric sensitivity analysis comes from varying non-continuous parameters; unless occupant restraints are applied, the wheelchair passenger will be ejected and impact the LATV interior. This finding supports our previous observation that the disuse of occupant restraints results in wheelchair passenger ejection during emergency braking maneuvers. We also found that lateral wheelchair excursions were substantially less than fore/aft wheelchair excursions, as tendency for lateral movement was limited by the folded-seat beneath the window which served as a barrier. This finding provides preliminary evidence that inclusion of a side barrier in close proximity to the wheelchair on the aisle side of a passenger containment station could indeed serve to limit lateral wheelchair excursions into the aisle.

A summary of recommendations for stakeholders invested in wheelchair passenger safety in LATV transit is shown in Table 1. 


\begin{tabular}{|c|c|}
\hline Stake holder & Recommendation \\
\hline \multirow{5}{*}{ Transit Agencies } & Require wheelchair passengers to apply wheelchair brakes \\
\hline & Require wheelchair passengers to turn off power wheelchairs \\
\hline & Require correct employment of all four tiedowns to secure the wheelchair \\
\hline & Require correct use of the lap and shoulder belt \\
\hline & Additional training and monitoring of LATV operators with regard to proper WTORS use \\
\hline \multirow{5}{*}{ Wheelchair Passengers } & Apply wheelchair brakes \\
\hline & Turn off power wheelchairs \\
\hline & Request all four tiedowns be used correctly to secure the wheelchair \\
\hline & Request to be restrained correctly using the lap and shoulder belt \\
\hline & $\begin{array}{l}\text { Ask for / perform regular manual wheelchair brake inspections, assuring brake mounting hardware } \\
\text { is securely clamped to the wheelchair frame }\end{array}$ \\
\hline \multirow{2}{*}{ WTORS Manufacturers } & Advance alternative WTORS systems with special emphasis on the ease-of-use \\
\hline & Design retractors suitable for use in low-g $(<1 \mathrm{~g})$ environments \\
\hline Wheelchair Manufacturers & Improve brake design for manual transit wheelchairs \\
\hline \multirow{2}{*}{$\begin{array}{l}\text { Physical and Ocuppational } \\
\text { Therapists, Wheelchair } \\
\text { Suppliers }\end{array}$} & $\begin{array}{l}\text { Perform regular manual wheelchair brake inspections, assuring brake mounting hardware is } \\
\text { securely clamped to the wheelchair frame }\end{array}$ \\
\hline & $\begin{array}{l}\text { Assure appropriate wheelchair wheelbase for user's balancing ability during wheelchair perscription } \\
\text { process }\end{array}$ \\
\hline
\end{tabular}

Table 1. Recommendations for stakeholders invested in wheelchair passenger safety in LATV transit.

\section{Limitations and Future Work}

Our case study was somewhat limited by low resolution video footage and/or a limited number of cameras with limited view angles. Future work could be conducted with additional high-resolution cameras and view angles may provide more detailed information regarding wheelchair passenger transit on LATVs.

Another major limitation of our study is that an ATD was used to represent the wheelchair passenger, which may not be an ideal representation. The ATD was designed based on the $50^{\text {th }}$ percentile anthropometric measures of the general male population; these measures may not be the same for the wheelchair passenger population. 
Additionally, the ATD was developed and validated for use in a high-g (20g) crash environment, while our study was conducted in a low-g $(<1 \mathrm{~g})$ setting. The ATD's joint properties may be too stiff to accurately simulate actual wheelchair passenger response in a low-g environment. However, as surrogate humans based on the wheelchair population and/or surrogate humans validated for low-g environments have not yet been developed, the ATD used in this study is a reasonable first step in representing a wheelchair passenger. Future work could involve the design of a mid-sized ATD based on different wheelchair user population subsets. One subset may be obese wheelchair users, while another subset may be amputee wheelchair users with different segment mass distribution or a subset of wheelchair users with a spinal cord injury. Having an ATD that is representative of a specific subset of wheelchair users could allow us to determine the relative contributions of factors such as body mass, postural stability, muscle tone, etc. to risks associated with adverse events in transit.

The wheelchair used in our study was an ultra-light, $x$-braced frame, manual wheelchair; other wheelchairs may have produced different tiedown loading and wheelchair accelerations. We attempted to address this limitation with the parametric sensitivity analysis by modeling a worst-case scenario of a heavy power-base wheelchair.

Our study also failed to provide wheelchair accelerations during normal driving maneuvers and ATD pelvic accelerations during emergency driving, because of malfunctioning accelerometers. These data would have provided additional measures describing the kinematic environment experienced by a wheelchair and wheelchair passenger. Furthermore, right rear tiedown loading data was limited because the associated load cell failed during testing. The number of emergency maneuvers for the 
WTORS disuse and misuse conditions was limited because of threat of equipment damage from ATD ejections. Restrictions on time and use of the LATV did not allow us to repeat testing to gather this information.

Some data were not measurable during LATV emergency driving because of limited accelerometer range $(0 \pm 6 \mathrm{~g})$ such as wheelchair and ATD accelerations as the wheelchair or ATD impacted the LATV interior. Future studies should measure high-g impact accelerations that may be used to assess injury risk as the ATD impacts the LATV interior.

The manual wheelchair brakes used in our study were compact scissor wheel locks. Although this brake design is common on manual wheelchairs, other brake designs may have produced different results. We did not reaffirm that the brake mounting hardware was securely clamped to the wheelchair frame after each trial, so the brakes' effectiveness may have been diminished in later trials. Additionally, the wheelchair tipping could have influenced how securely the brake mounting hardware was clamped to the wheelchair frame in subsequent trials. Future work should focus on investigating the robustness of various manual wheelchair brake mounting hardware designs for various wheelchair makes and models as it relates to wheelchair stability in LATV transit.

Our computer model was validated for LATV emergency braking maneuvers, but it may not be suitable for all other LATV maneuvers, such as acceleration or pure turning maneuvers. The ATD and wheelchair dynamics in our model are specific to low-g LATV braking/left turn maneuvers. LATV accelerations and pure turning maneuvers would produce different ATD and wheelchair dynamics that may not be accurately predicted by 
our model. Future research should determine the model's performance in other LATV maneuvers.

Furthermore, our model validation was limited to only one experimental LATV emergency combination braking/left-turn maneuver. Additional experimental maneuvers would have provided a more robust model validation. However, limited resources (mainly LATV and LATV operator time) prevented us from collecting additional usable data to be included for model validation. Nonetheless, we consider our model a good predictor of ATD and wheelchair dynamics in LATV emergency braking/left-turn maneuvers.

Our computer model did not include LATV interior bulkheads (except the foldedseat beneath the window, which served as a side barrier). Future work should focus on an expansion of the baseline model to feature the front bulkhead. In the parametric sensitivity analysis, we found frequent ATD ejection resulting from varying WTORS configurations. The new model featuring the front bulkhead could be used to assess injury risk as the ATD impacts the front bulkhead.

A major limitation associated with the parametric sensitivity analysis is that the sensitivity index (SI) was only calculated for continuous variable parameters (such as height, mass, and stiffness). Variation of categorical variable parameters such as WTORS configurations produced important results (ATD ejections) that cannot be directly compared to the continuous parameters using the SI.

Rationale for varying wheelchair CG height and wheelchair mass were based on a study ${ }^{16}$ published in 1997. While more than a decade has passed since the publication of these data and today's wheelchairs may have different CG height and mass, a standard 
literature search failed to produce more recently published studies describing these wheelchair characteristics. Future studies could focus on providing an updated depiction of key wheelchair parameters that may influence wheelchair transportation.

In the parametric sensitivity analysis, the parameters were varied independently; varying two or more parameters together may have produced different results. The parametric sensitivity analysis of wheelchair mass increase and wheelchair CG height variations were not accompanied by geometric changes to the wheelchair (e.g. different wheels, etc.). None of these changes would have been possible without extensive changes to the model, which may have reduced its validity. Future research could include validation of a model with a power wheelchair seated passenger.

This study is specific to evaluating 4-point, strap-type WTORS variations. The findings from this study may not be directly applicable to the evaluation of alternative wheelchair securement and/or containment devices, such as the auto-docking system, and the rear- and front-facing containment stations. Future work could include development of a new computer model to investigate these alternative approaches to wheelchair securement.

Another limitation in this study is the use of only one LATV make and model; different LATVs may have produced different LATV accelerations, ultimately yielding different WTORS loading, wheelchair accelerations and ATD accelerations. We attempted to address this by including an important LATV parameter in our parametric sensitivity analysis: LATV suspension stiffness. The variation of LATV suspension stiffness was based on published differences in vertical acceleration at different locations in the LATV ${ }^{50}$. Ideally, a future study could include a range of published suspension 
stiffnesses in the parametric sensitivity analysis. We attempted to identify this information for the parametric sensitivity analysis but several attempts to obtain it from the LATV manufacturer were unsuccessful. 


\section{REFERENCES}

1. Shaw, G., Wheelchair rider risk in motor vehicles: a technical note. Journal of Rehabilitation Research and Development, 2000. 37(1): p. 89-100.

2. Shaw, G., Investigation of large transit vehicle accidents and establishing appropriate protection for wheelchair riders. Journal of Rehabilitation Research and Development, 2008. 45(1): p. 85-108.

3. Shaw, G. and Gillispie, T., Appropriate protection for wheelchair riders on public transit buses. Journal of Rehabilitation Research and Development, 2003. 40(4): p. 309-320.

4. National Highway Traffic Safety Administration, Research Note: Wheelchair Users Injuries and Deaths Associated with Motor Vehicle related Incidents, 1997, U.S. Department of Transportation: Washington, DC.

5. Fitzgerald, S., Songer, T., Rotko, K., and Karg, P., Motor vehicle transportation use and related adverse events among persons who use wheelchairs. Assistive technology: the official journal of RESNA, 2007. 19(4): p. 180.

6. Songer, T., Fitzgerald, S., and Rotko, K. The injury risk to wheelchair occupants using motor vehicle transportation. 48th Annual Proceedings, Association for the Advancement of Automotive Medicine Conference Proceedings, September, 2004.

7. Frost, K. and Bertocci, G., Retrospective review of adverse incidents involving passengers seated in wheeled mobility devices while traveling in large accessible transit vehicles. Medical Engineering and Physics, 2010. 32(3): p. 230-236.

8. Americans with Disabilities Act (ADA), Transportation Services for Individuals with Disabilities, ADA, 49 CFR Part 37 Federal Register, 1990.

9. Foreman, C. and Hardin, J., The Challenges of Wheelchair Securement: Searching For Solutions, American Public Transportation Association (APTA), 2002.

10. Buning, M., Getchell, C., Bertocci, G., and Fitzgerald, S., Riding a bus while seated in a wheelchair: A pilot study of attitudes and behavior regarding safety practices. Assistive Technology, 2007. 19(4): p. 166-179.

11. Zaworski, J., Hunter-Zaworski, K., and Baldwin, M., Bus dynamics for mobilityaid securement design. Assistive Technology, 2007. 19(4): p. 200-09.

12. Wretstrand, A., Petzäll, J., and Ståhl, A., Safety as perceived by wheelchair-seated passengers in special transportation services. Accident Analysis and Prevention, 2004. 36(1): p. 3-11.

13. Americans with Disabilities Act (ADA), Accessibility Specifications for Transportation Vehicles, ADA, 49 CFR Part 38 Federal Register, 1990. 
14. Society of Automotive Engineers, SAE J2249: Wheelchair Tiedown and Occupant Restraint Systems for Use in Motor Vehicles, 1999: Warrendale, PA.

15. Wolf, P., van Roosmalen, L., and Bertocci, G., Wheelchair tiedown and occupant restraint system issues in the real world and the virtual world: combining qualitative and quantitative research approaches. Assistive Technology, 2007. 19(4): p. 188.

16. Bertocci, G., Karg, P., and Hobson, D., Wheeled mobility device database for transportation safety research and standards. Assistive Technology, 1997. 9: p. 102-115.

17. van Roosmalen, L., Karg, P., Hobson, D., Turkovich, M., and Porach, E., User evaluation of three wheelchair securement systems in large accessible transit vehicles. Journal of Rehabilitation Research and Development, 2011. 48(7): p. 823.

18. Hobson, D. and van Roosmalen, L., Towards the next generation of wheelchair securement--development of a demonstration UDIG-compatible wheelchair docking device. Assistive Technology, 2007. 19(4): p. 210-22.

19. International Standards Organization, ISO 10542-2: Technical systems and aids for disabled or handicapped persons - Wheelchair tiedown and occupant-restraint systems, International Standard Organization, 2001: Geneva, Switzerland.

20. International Standards Organization, ISO 10865-1: Assistive products for persons with disability - Wheelchair containment and occupant retention systems for motor vehicles designed for use by both sitting and standing passengers - Part 1: Systems for rearward facing wheelchair-seated passengers, International Standards Organization, 2011: Geneva, Switzerland.

21. Frost, K., Bertocci, G., and Salipur, Z., Wheelchair Securement and Occupant Restraint System (WTORS) Practices in Public Transit Buses. Assistive Technology Journal, 2013. 25(1): p. 16-23.

22. International Standards Organization, ISO 10865-2 (DRAFT): Assistive products for persons with disability - Wheelchair containment and occupant retention systems for motor vehicles designed for use by both sitting and standing passengers - Part 2: Systems for forward facing wheelchair-seated passengers, International Standards Organization, 2012: Geneva, Switzerland.

23. Linden, M. and Sprigle, S., Development of instrumentation and protocol to measure the dynamic environment of a modified van. Journal of Rehabilitation Research \& Development, 1996. 33(1): p. 23-29.

24. Sprigle, S. and Linden, M., Accelerations experienced by wheelchair users with spinalcord injury in a moving van. Technology and Disability, 1996. 5: p. 81-91.

25. Kamper, D., Parnianpour, M., Barin, K., Adams, T., Linden, M., and Hemami, H., Postural stability of wheelchair users exposed to sustained, external perturbations. Journal of Rehabilitation Research \& Development, 1999. 36(2): p. 121-132.

26. Kamper, D.G., Adams, T.C., Reger, S.I., Parnianpour, M., Barin, K., and Linden, M., A low-cost, portable system for the assessment of the postural response of wheelchair users to perturbations. IEEE Transactions on Rehabilitation Engineering, 1999. 7(4): p. 435-442.

27. Kamper, D.G., Adams, T.C., Reger, S.I., Parnianpour, M., Barin, K., and Linden, M.A., A technique for quantifying the response of seated individuals to dynamic 
perturbations. Journal of Rehabilitation Research \& Development, 2000. 37(1): p. 81-88.

28. International Standards Organization, ISO 7176/19: Wheeled mobility devices for use as seats in motor vehicles, International Standard Organization, 2008:

Geneva, Switzerland.

29. Turkovich, M., van Roosmalen, L., Hobson, D., and Porach, E., The Effect of

City Bus Maneuvers on Wheelchair Movement. Journal of Public Transportation 2011. 14(3): p. 147-169.

30. Bertocci, G., Hobson, D., and Digges, K., Development of Transportable Wheelchair Design Criteria Using Computer Crash Simulation. IEEE Transactions on Rehabilitation Engineering, 1996. 4(3): p. 10.

31. Fuhrman, S., Karg, P., and Bertocci, G., Characterization of pediatric wheelchair kinematics and wheelchair tiedown and occupant restraint system loading during rear impact. Medical Engineering and Physics, 2009.

doi:10.1016/j.medengphy.2009.03.006.

32. Leary, A. and Bertocci, G. Design Criteria For Manual Wheelchairs Used As Motor Vehicle Seats Using Computer Simulation. RESNA Conference Proceedings, Reno, NV, June 2001.

33. Manary, M. and Ritchie, N., Personal Communication, 2007, University of Michigan Transportation Research Institute (UMTRI): Ann Arbor, MI.

34. Salipur, Z. and Bertocci, G., Wheelchair tiedown and occupant restraint system loading associated with adult manual transit wheelchair in rear impact. Journal of Rehabilitation Research \& Development, 2010. 47(2): p. 143-150.

35. Blower, D., Woodrooffe, J., and Scheider, L. Characterization of transit-bus accidents resulting in passenger injuries for use in developing alternative methods for transporting wheelchair-seated travelers. International Truck and Bus Safety and Security Symposium, Conference Proceedings, Alexandria, Virginia, USA,

36. ANSI/RESNA, WC19: Wheelchairs Used as Seats in Motor Vehicles, American National Standards Institute (ANSI)/Rehabilitation Engineering and Assistive Technology Society of North America (RESNA), 2000.

37. van Roosmalen, L. and Turkovich, M. Selecting an anthropomorphic test device for low acceleration wheelchair transportation testing. RESNA Conference Proceedings, Baltimore, MD, June 2012.

38. Salipur, Z., Frost, K., and Bertocci, G., Investigation of wheelchair instability during transport in large accessible transit vehicles. Journal of Rehabilitation Research \& Development, 2012. 49(6): p. 935-48.

39. Bertocci, G., Szobota, S., Hobson, D., and Digges, K., Computer Simulation and Sled Test Validation of a Powerbase Wheelchair and Occupant Subjected to Frontal Crash Conditions. IEEE Transactions on Rehabilitation Engineering, 1999. 7(2): p. 10.

40. Ha, D., Bertocci, G.E., and Jategaonkar, R., Development and Validation of a Frontal Impact 6-Year-Old Occupant and Wheelchair Computer Model. Assistive Technology, 2007. 19(4): p. 223-238.

41. Dsouza, R. and Bertocci, G., Development and validation of a computer crash simulation model of an occupied adult manual wheelchair subjected to a frontal 
impact. Medical Engineering and Physics, 2009. doi:

10.1016/j.medengphy.2009.01.008.

42. Manary, M. and Schneider, L. Wheelchair Seatback Loads and Deflections in Rear Impacts. RESNA Conference Proceedings, Orlando, FL, June 2007.

43. Paskoff, G., Transportation of Wheelchair Users: An Assessment of Neck Injury Risk During Rear Collisions, Thesis for M.S. in Biomedical Engineering, Biomedical Engineering, School of Engineering and Applied Science, University of Virginia, 1995.

44. Salipur, Z. and Bertocci, G., Development and validation of rear impact computer simulation model of an adult manual transit wheelchair with a seated occupant. Medical Engineering \& Physics, 2010. 32(1): p. 66-75.

45. Netherlands Organization for Applied Scientific Research (TNO) Automotive Safety Solutions division (TASS), 'MADYMO Theory Manual, Release 7.0'. 2008, Delft, Netherlands.

46. Netherlands Organization for Applied Scientific Research (TNO) Automotive Safety Solutions division (TASS), 'MADYMO Model Manual, Release 7.0'. 2008, Delft, Netherlands.

47. Larsson, A., Pettersson, M., and Svensson, H., User Manual CurveAnalyzer v.1.0, Chalmers University of Technology, 2003.

48. Pipcorn, B. and Eriksson, M. A Method to Evaluate the Validity of Mathematical Models. MADYMO European User's Meeting Conference Proceedings, Brussels, Belgium, 2003.

49. Hunter-Zaworski, K.M. and Zaworski, R.J., Assessment of Rear Facing Wheelchair Accommodation on Bus Rapid Transit, Final Report for Transit IDEA Project 38, Innovations Deserving Exploratory Analysis (IDEA) Programs, Transportation Research Board, 2005.

50. Hunter-Zaworski, K.M. and Zaworski, J.R., Vehicle Dynamics Study of Smaller Public Transportation Vehicles Used as Paratransit or Shuttle Vehicles, in Transportation Research Board (TRB) 90th Annual Meeting 2011: Washington, DC. 


\section{CURRICULUM VITAE}

April 3, 2013

\section{Zdravko Salipur, M.Eng.}
Business Address: University of Louisville
Mechanical Engineering
Health Science Campus
Research Tower - Room 204
Louisville, KY, 40202
Telephone: $\quad$ Cell: (502) 296-7826 Office: (502) 852-0279
Email:
z.salipur@louisville.edu
zsalipur@hotmail.com

\section{Education}

2008 - $2013 \quad$ University of Louisville

Ph.D. Mechanical Engineering (exp. 05/2013)

(Dean's Citation Award, summa cum laude, GPA: 3.93/4.0)

2006 - $2008 \quad$ University of Louisville

M.Eng. Mechanical Engineering (2008)

(summa cum laude, GPA: 4.0/4.0)

2001 - $2005 \quad$ University of Louisville

B.S. Mechanical Engineering (2005)

(magna cum laude, GPA: 3.7/4.0)

$\underline{\text { Academic }}$

\section{Positions}

01/2006 - present Injury Risk Assessment and Prevention (iRAP) Laboratory, University of Louisville, Graduate Research Assistant Conduct research in Injury Biomechanics, Wheelchair Transportation Safety

- Consequences of wheelchair tiedown and occupant restraint system practices on wheelchair passenger safety in fixed-route transit (Switzer Fellowship Project funded by NIDRR , Role: PI) 
0 retrospective video review of wheelchair passenger related adverse events in public buses

o planned, coordinated, and conducted in-field experimental testing to evaluate wheelchair passenger related adverse events in public buses

0 development and validation of low-g computer simulation wheelchair model using MADYMO

0 parametric sensitivity analysis to yield wheelchair and wheelchair tiedown manufacturer design guidelines

- Assessment and Monitoring of Wheelchair Transportation Activities and Incidents on Public Buses (Project P2, Rehabilitation Engineering Research Center on wheelchair transportation safety)

- Investigate wheelchair and wheelchair tiedown integrity during rear impact collisions using various techniques, including sled impact testing and computer simulation. Project funded by Paralyzed Veterans of America (PVA) grant.

- planned, coordinated, and conducted rear impact sled tests at the University of Michigan

0 development and validation of computer simulation wheelchair model using MADYMO

o parametric sensitivity analysis to yield wheelchair and wheelchair tiedown manufacturer design guidelines

04/2009 - 04/2010 Frazier Rehabilitation Center, Louisville, KY

Intern at the Assistive Technology (AT) Clinic

- Clinical experience with Rehabilitation Engineering

- Assistive Technology evaluation and delivery

05/2008 - 07/2008 University of Michigan Transportation Research Institute (UMTRI), Biosciences Division, University of Michigan, Ann Arbor, MI

Graduate Student Intern

- Primary project: assembling first draft of annex to ISO 7176-19 standard titled: "Wheeled mobility devices for use as seats in motor vehicles - Forward facing mobility aids in rear impact"

- Aid in daily operation of running an impact sled laboratory

- Helped with in-depth crash investigations of motor vehicle accidents.

- Mentored undergraduate research student responsible for developing instrumentation of surrogate seating system.

08/2005 - 12/2005 Investigation of Conveyer Belt Pulley for Cylicron Inc.

- Mentor: Dr. Keith Sharp 
- Undergraduate Capstone Project. As a result of successful project completion, Cylicron awarded \$2000 grant to the University of Louisville

$\underline{\text { Non-Academic }}$

$09 / 2005-01 / 2006$

Alcoa, Louisville, KY

Part-Time Mechanical Engineering Co-op Supported chief maintenance engineer in assuring smooth, continuous plant operations

- Daily responsibilities:

- Develop and provide CAD drawings using Auto CAD

o Manage kick-outs to assure continuous and complete shipment of customer products.

- Major Projects undertaken:

0 preventive maintenance program for a range of equipment in entire plant

o winter shutdown maintenance on laminators and slitters

08/2003 - 06/2005 Vacuum Depositing Inc. (VDI), Louisville, KY

Full-Time / Part-Time Engineering Co-op

Supported president and vice president of Engineering in assuring smooth, continuous plant operations

- Daily responsibilities:

o information technology (IT) support for entire facility including network installation and computer installation/upgrades (familiar with small business networking)

o database support in MS Access through data entry management and development/modification of reports and queries

- Major projects undertaken:

0 design and implementation of class $10 \mathrm{~K}$ clean-room to increase quality control

0 preventive maintenance program for a range of equipment in entire plant

o Aided ISO manager in ISO recertification by establishing in-house records required for compliance

o Rebuilding MS Access database for more efficient and accurate production oversight and quality control

o Rebuilding and expansion of IT network to accommodate new MS Access database

o Vapor coater remodeling with newest webbing technology (including chilled drum), wire drives, and density control system. 


\title{
Professional/Scientific Societies and Extracurricular Activities
}

\author{
2007- present \\ ANSI/RESNA Standards Committee on Wheelchairs \\ and Transportation (COWHAT) - Voting Member \\ 2006 - present \\ Pi Tau Sigma, Mechanical Engineering Society - Member \\ 2004 - present \\ Tau Beta Pi Engineering Honor Society - Member \\ 2002 - present \\ American Society of Mechanical Engineers (ASME) - Member \\ $2006-2008$ \\ Biomedical Engineering Society (BMES) - President, Member \\ $2005-2007$ \\ Society of Automotive Engineers (SAE) Formula - Member \\ $2002-2004$ \\ University of Louisville Intramural Soccer - Captain \\ $2000-2001$ \\ Tutor and Guide for Big Brother Program - Volunteer

\section{Honors and Awards} \\ 2013 \\ Graduate Dean's Citation Award - For significant \\ accomplishments during graduate career at the University of \\ Louisville. Designed to honor top 10\% of graduates in each \\ graduate program.
}

2012

Second Place, Student Research Presentations, American Society of Mechanical Engineers (ASME), Louisville Professional Chapter, Louisville, KY.

Honorable Mention ( $\mathrm{PhD}$ Level) at the Engineering Exposition (EExpo) Poster Presentation. University of Louisville Speed Scientific School, Louisville, KY.

Winner of the Student Scientific Paper Competition at annual conference of Rehabilitation Engineering and Assistive Technology Society of North America (RESNA), Phoenix, AZ.

Elisabeth M. And Winchell M. Parsons Graduate Scholarship competitive, national award granted by the American Society of Mechanical Engineers (ASME) Auxiliary to top 1-2 students in U.S. pursuing a Ph.D. in Mechanical Engineering.

University of Louisville Grosscurth Fellowship - fellowship awarded by the J.B. Speed School of Engineering for pursuing a Doctorate of Philosophy in engineering. 
Marjorie Roy Rothermel Graduate Scholarship - competitive, national award granted by the American Society of Mechanical Engineers (ASME) Auxiliary to top eight graduate students in U.S.

Burton Scholarship - competitive award granted by University of Louisville, J. B. Speed School of Engineering to one undergraduate student.

\section{Grants Awarded}

Name: Switzer Research Fellowship Grant

Source: National Institute on Disability and Rehabilitation Research (NIDRR), Department of Education

Role: Principal Investigator (PI)

Title: Consequences of wheelchair tiedown and occupant restraint system practices on wheelchair passenger safety in fixed-route transit

Amount: \$65,000

Grant Number: H133F100032

Dates: $10 / 01 / 2010-03 / 31 / 2012$

\section{Publications}

\section{Peer-reviewed Journal Articles}

Salipur Z., Bertocci G. Manual Wheelchair Excursions Resulting From Wheelchair Tiedown and Occupant Restraint System Misuse during Large Accessible Transit Vehicle Normal Driving Maneuvers. Manuscript in preparation.

Salipur Z., Bertocci G. Development and Validation of Computer Simulation Model of an Adult Manual Transit Wheelchair with a Seated Occupant in Large Accessible Transit Vehicle under Emergency Braking Conditions. Manuscript in preparation.

Salipur Z., Bertocci G. Influence of Transit Bus Normal Driving Conditions on Wheelchair Occupants and

WTORS loads. Submitted for publication to the Journal of Rehabilitation Research and Development on March, 9, 2013.

Frost K., Bertocci G., Salipur Z. Wheelchair Securement and Occupant Restraint System (WTORS) Practices in Public Transit Buses. Assistive Technology Journal. 25(1). 2013.

Salipur Z., Frost K., Bertocci G. Investigation of Wheelchair Instability During Transport in Large Accessible Transit Vehicles. Journal of Rehabilitation Research and Development. 49(6). 2012. 
Salipur Z., Bertocci G. Wheelchair Tiedown and Occupant Restraint Loading Associated with an Adult Manual Transit Wheelchair in Rear Impact. Journal of Rehabilitation Research and Development. 47(2). 2010.

Salipur Z., Bertocci G. Development and Validation of Rear Impact Computer Simulation Model of an Adult Manual Transit Wheelchair with a Seated Occupant. Medical Engineering and Physics. 32(1). 2010.

\section{Peer-reviewed Conference Papers}

Salipur Z, Bertocci G. Development and Validation of Rear Impact Computer Simulation Model of an Adult Manual Transit Wheelchair with a Seated Occupant. Proceedings of the Annual RESNA 2008 Conference. June 2008.

Salipur Z, Bertocci G, Manary M, Ritchie N. Wheelchair Tiedown and Occupant Restraint System Loading Associated with an Adult Manual ANSI WC-19 Transit Wheelchair with a Seated $50^{\text {th }}$ percentile ATD Exposed to Rear Impact. Proceedings of the Annual RESNA 2007 Conference. June 2007.

Manary M, Bezaire B, Bertocci G, Salipur Z, Schneider L. Crashworthiness of ForwardFacing Wheelchairs under Rear Impact Conditions. Proceedings of the Annual RESNA 2007 Conference. June 2007.

\section{Presentations / Dissemination}

$04 / 2012$

Biomedical Engineering Society (BMES) Research Showcase, University of Louisville, Red Barn, Louisville, KY.

Poster - Kinematic Environment and Consequences of Wheelchair Tiedown and Occupant Restraint System Practices on Wheelchair Passenger Safety in Fixed-route Transit

$03 / 2012$

Engineering Exposition (E-Expo), University of Louisville Speed Scientific School, Louisville, KY.

Poster - Kinematic Environment and Consequences of Wheelchair Tiedown and Occupant Restraint System Practices on Wheelchair Passenger Safety in Fixed-route Transit

$02 / 2012$

American Society of Mechanical Engineers (ASME), Louisville Professional Chapter Meeting, Louisville, KY.

Platform Presentation - Kinematic Environment and Consequences of wheelchair tiedown and occupant restraint system practices in fixed-route transit 
$12 / 2011$

$06 / 2008$

$03 / 2008$

$10 / 2007$

$06 / 2007$

$06 / 2007$

$04 / 2007$

$03 / 2007$
Mary E. Switzer Research Fellowship Seminar National Institute on Disability and Rehabilitation Research (NIDRR), Arlington, VA.

Platform Presentation - Consequences of wheelchair tiedown and occupant restraint system practices on wheelchair passenger safety in fixed-route transit

Annual Conference of Rehabilitation Engineering and Assistive Technology Society of North America (RESNA), Washington, D.C.

Poster - Development and Validation of Rear Impact Computer Simulation Model of an Adult Manual Transit Wheelchair with a Seated Occupant

Engineering Exposition (E-Expo), University of Louisville Speed Scientific School, Louisville, KY.

Poster - Development and Validation of Rear Impact Computer Simulation Model of an Adult Manual Transit Wheelchair with a Seated Occupant

Alumni Association Homecoming Banquette, University of Louisville Speed Scientific School, Louisville, KY.

Poster - Transit Wheelchair Performance and Tiedown Loading in Rear Impact

Annual Conference of Rehabilitation Engineering and Assistive Technology Society of North America (RESNA), Phoenix, AZ. Poster - Wheelchair Tiedown and Occupant Restraint System Loading Associated with an Adult Manual ANSI WC19 Transit Wheelchair with a Seated 50th Percentile ATD Exposed to Rear Impact

Annual Conference of Rehabilitation Engineering and Assistive Technology Society of North America (RESNA), Phoenix, AZ.

Platform Presentation - Wheelchair Tiedown and Occupant Restraint System Loading Associated with an Adult Manual ANSI WC19 Transit Wheelchair with a Seated 50th Percentile ATD Exposed to Rear Impact

Biomedical Engineering Society (BMES), University of Louisville Student Chapter - Professional Development Forum Platform Presentation - Transit Wheelchair Performance and Tiedown Loading in Rear Impact

Engineering Exposition (E-Expo), University of Louisville Speed Scientific School, Louisville, KY. 
$03 / 2007$

Summer 2012

Summer 2010

Spring 2010

Fall 2009

Summer 2009

Spring 2009
Poster - Transit Wheelchair Performance and Tiedown Loading in Rear Impact

American Society of Mechanical Engineers (ASME) / American

Society of Heating, Refrigerating, and Air-Conditioning Engineers (ASHRAE) Louisville Professional Chapters, Professional Development, Louisville, KY.

Platform Presentation - Transit Wheelchair Performance and

Tiedown Loading in Rear Impact

\section{Teaching / Mentoring}

Instructor for undergraduate course in Mechanical Engineering Department, University of Louisville Course number and title: ME 206: Engineering Mechanics II: Dynamics

(Evaluations: 4.36/5.0)

Instructor for undergraduate course in Health and Sports Sciences Department, University of Louisville Course number and title: HSS 387: Biomechanics (Evaluations: 4.35/5.0)

Instructor for undergraduate course in Health and Sports Sciences Department, University of Louisville Course number and title: HSS 387: Biomechanics (Evaluations: 4.04/5.0)

Instructor for undergraduate course in Health and Sports Sciences Department, University of Louisville Course number and title: HSS 387: Biomechanics (Evaluations: 3.30/5.0)

Instructor for undergraduate course in Health and Sports Sciences Department, University of Louisville Course number and title: HSS 387: Biomechanics (Evaluations: 4.46/5.0)

Teaching Assistant (TA) for Mechanical Engineering graduate level course: Injury Biomechanics (ME 675), University of Louisville

Responsibilities: lecturing/teaching, grading, writing exams

Spring 2007 - Fall 2009 Jefferson County Public Schools Adult Education - German for Beginners 
Summer 2008

Graduate student mentor for undergraduate research student at University of Michigan Transportation Research Institute (UMTRI). Project: Developing instrumentation of surrogate seating system.

Spring 2008

Graduate student mentor for senior Capstone Design Project Group in Mechanical Engineering, University of Louisville. Project: Designing Assistive Technology: A Basketball Shooter to allow wheelchair users with limited mobility to play basketball

\section{Expert Witness Work}

Wheelchair Biomechanics: Investigation of Power Wheelchair (Operational Feasibility) Client: McMasters Keith Law Offices (Sara Clark Davis), The Forum at Brookside. May 2011 - Jan 2012.

\section{Other Skills}

Computer $\quad$ Windows, Mac OS X, MS Office Pro, Visual BASIC (VB), MATLAB, C++, AutoCAD, Solid Edge, ANSYS, HTML, Pascal, QBASIC, ATB 3 ${ }^{\mathrm{i}}$, MADYMO, LabVIEW, eVART (Cortex)

$\begin{array}{ll}\text { Material Testing } & \text { MTS, ADMET } \\ \text { Machining/ } & \begin{array}{l}\text { Basic tooling (Lathe, and Mill) } \\ \text { Maintenance }\end{array} \\ \begin{array}{l}\text { Basic maintenance of various industrial equipment including vapor } \\ \text { coaters, sputterers, laminators, and slitters }\end{array} \\ \text { Languages } & \begin{array}{l}\text { Multilingual (oral and written) in English, German, and Serbo- } \\ \text { Croatian }\end{array}\end{array}$

References Available upon request 